\title{
FastBit Reference Manual
}

\author{
$-\mathrm{a} 0.7-$ \\ Kesheng Wu \\ Scientific Data Management \\ Lawrence Berkeley National Lab \\ August 2007 \\ Report Number: LBNL/PUB-3192
}




\section{Contents}

1 Overview of FastBit IBIS Implementation 1

2 FastBit Namespace Documentation $\quad 3$

3 FastBit Class Documentation $\quad 14$

4 FastBit File Documentation $\quad 259$

\section{Overview of FastBit IBIS Implementation}

\subsection{Introduction}

An index in a database system is a data structure that utilizes redundant information about the base data to speed up common searching and retrieval operations. Most commonly used indexes are variants of B-trees, such as B+-tree and $\mathrm{B} *$-tree. FastBit implements a set of alternative indexes call compressed bitmap indexes. Compared with B-tree variants, these indexes provide very efficient searching and retrieval operations by sacrificing the efficiency of updating the indexes after the modification of an individual record.

In addition to the well-known strengths of bitmap indexes, FastBit has a special strength stemming from the bitmap compression scheme used. The compression method is called the Word-Aligned Hybrid (WAH) code. It reduces the bitmap indexes to reasonable sizes and at the same time allows very efficient bitwise logical operations directly on the compressed bitmaps. Compared with the well-known compression methods such as LZ77 and Byte-aligned Bitmap code (BBC), WAH sacrifices some space efficiency for a significant improvement in operational efficiency. Since the bitwise logical operations are the most important operations needed to answer queries, using WAH compression has been shown to answer queries significantly faster than using other compression schemes.

Theoretical analyses showed that WAH compressed bitmap indexes are optimal for one-dimensional range queries. Only the most efficient indexing schemes such as B+-tree and B*-tree have this optimality property. However, bitmap indexes are superior because they can efficiently answer multi-dimensional range queries by combining the answers to one-dimensional queries.

\subsection{Key Components}

FastBit process queries on one table at a time. Currently, there are two sets of interfaces for query processing, one more abstract and the other more concrete. The more abstract interface is represented by the class $i \mathrm{~b} i \mathrm{~s}:: \mathrm{table}$ and the more concrete interface is represented by the class ibis : : part. A table (with rows and columns) is divided into groups of rows called data partitions. Each data partition is stored in a column-wise organization known as vertical projections. At the abstract level, queries on a table produces another table in the spirit of the relational algebra. At the concrete level, the queries on data partitions produce bit vectors representing rows satisfying the user specified query conditions.

\subsubsection{Operations on Tables}

The main class representing this interface is ibis: table. The main query function of this class is ibis: : table: : select, whose functionality resembles a simplified form of the SELECT statement from the SQL language. This function takes two string as arguments, one corresponds to the select clause in SQL and the other corresponds to the where clause. In the following, we will call them the select clause and the where clause and discussion the requirements and restriction on these clauses.

The select clause passed to function ibis: : table: : select can only contain column names separated by comma (,). Aggregate operations such as MIN, MAX, AVG or SUM, are supported through another function named ibis: : table: : groupby. A group-by operation normally specified as one SQL statement needs to be split into 
two steps, one to select the values and the other to perform the aggregation operations. We've taken this approach to simplify the implementation. Because these aggregation operations are not directly supported by bitmap indexes, therefore, they are not essential to demonstrate the effectiveness of the bitmap indexes.

The where clause passed to function ibis: : table: : select can be a combination of range conditions connected with logical operators such as AND, OR, XOR, and NOT. Assuming that temperature and pressure are names of two columns, the following are valid where clauses,

temperature > 10000

pressure between 10 and 100

temperature $>10000$ and $50<=$ pressure and sin(pressure/8000) < sqrt (abs (temperature)

The class ibis: : table also defines a set of functions for computing histograms of various dimensions, ibis::table.

Using FastBit, one can only append new records to a table. These operations for extending a table is defined in the class ibis: : tablex.

For most fixed-sized data, such as integers and floating-point values, FastBit functions expects raw binary data and also store them as raw binary, therefore the data files and index files are not portable across different platforms. This is common to both ibis: : table interface and ibis: :part interface. However, one difference is that ibis: : table handles string values as std: : vector<std: :string $>$, while the lower level interface ibis: :part handles strings as raw char* with null terminators.

\subsubsection{Operations on Data Partitions}

The two key classes for query processing on a data partition are ibis: :part and ibis: :query, where the first represents the user data (or base data) and the second represents a user query. An ibis: : part is primarily a container of ibis: : column objects and some common information about the columns in a data partition. The class ibis: : column has two specialization for handling string values, ibis: :category for categorical values and ibis: : text for arbitrary text strings.

The user query is represented as an ibis: :query object. Each query is associated with one ibis: :part object. The functions of the query class can be divided into three groups, (1) specifying a query, (2) evaluating a query, and (3) retrieving information about the hits. The queries accepted by FastBit are a subset of the SQL SELECT statement. Each query may have a WHERE clause and optionally a SELECT clause. Note that the FROM clause is implicit in the association with an ibis: : part. The WHERE clause is a set of range conditions joined together with logical operators, e.g., " $<$ code $>\mathrm{A}=5$ AND (B between 6.5 and 8.2 OR $\mathrm{C}>\operatorname{sqrt}(5 * \mathrm{D}))</$ code $>$." The SELECT clause can contain a list of column names and some of the four functions AVG, MIN, MAX and SUM. Each of the four functions can only take a column name as its argument. If a SELECT clause is omitted, it is assumed to be "SELECT count $(*)$." We refer to this type of queries as count queries since their primary purpose is to count the number of hits.

To evaluate a query, one calls either ibis: query: :estimate or ibis: query: :evaluate. After a query is evaluated, one may call various function to find the number of hits (ibis: :query: :getNumHits), the values of selected rows (ibis: query::getQualifiedInts, ibis: :query: :getQualifiedFloats, and ibis: query: :getQualifiedDoubles), or the bitvector that represents the hits (ibis: : query: : getHitvector).

\subsubsection{Indexes}

The indexes are considered auxiliary data, therefore even though they involve much more source files than ibis: :part and ibis: :query, they are not essential from a users point of view. In FastBit, the indexes are usually built automatically as needed. However, there are functions to explicitly force FastBit to build them through ibis: : table: :buildIndex, ibis: :part: :buildIndex and their variants.

Currently, all indexes are in a single class hierarchy with ibis: : index as the abstract base class. The most convenient way to create an index is calling the class function ibis: : index: :create. One can control what type of bitmap index to use by either specifying an index specification for a whole table by calling ibis: : table: : indexSpec, for a whole data partition by calling ibis: : part : : indexSpec, or for each individual column by calling ibis: :column: : indexspec. The index specification along with other metadata are written to a file named 
-part.txt in the directory containing the base data and the index files. The directory name is needed when constructing an ibis::part. This information may be indirectly provided through an RC file specified to the function ibis: : init.

\subsection{Acknowledgments}

The author gratefully acknowledges the support from Kurt Stockinger, Ekow Otoo and Arie Shoshani. They are crucial in establishing the foundation of the FastBit system and applying the software to a number of applications. Many thanks to the early users. Their generous feedbacks and suggestions are invaluable to the development of the software.

This work was supported by the Director, Office of Science, Office of Advanced Scientific Computing Research, of the U.S. Department of Energy under Contract No. DE-AC02-05CH11231.

\subsection{Additional Information}

Additional information available on the web at <http://sdm.lbl.gov/fastbit> or <http://lbl.gov/ kwu/fastbit>.

\section{FastBit Namespace Documentation}

\section{1 ibis Namespace Reference}

The current implementation of FastBit is code named IBIS and most data structures and functions are in the name space ibis.

\section{Classes}

- class ambit

The multi-level range based (cumulative) index.

- class bad_alloc

A specialization of std::bad_alloc.

- class bak

Maps each value to a lower prevision (decimal) values and use the the low precision value as center of the bin.

- class bak2

A variation on ibis: : bak, it splits each bin of ibis::bak in two, one for entries less than the mapped value and one for the entries that greater and equal to the mapped value.

- class bin

The equality encoded bitmap index with binning.

- class bitvector

A data structure to represent a sequence of bits.

- class bitvector64

A data structure to represent a sequence of bits.

- class bord

Class ibis::bord stores all its data in memory. 
- class bundle

The public interface of bundles.

- class bundle 0

The null bundle. It contains only a list of RIDs.

- class bundle1

The bundle with only one component.

- class bundles

The bundle with multiple components.

- class bylt

The two-level range-equality code.

- class category

A specialized low-cardinality text field.

- class colDoubles

A class to store double precision floating-point values.

- class colFloats

A class to store single precision float-point values.

- class collnts

A class to store integer values.

- class colLongs

A class to store integer values.

- class colUInts

A class to store unsigned integer values.

- class colULongs

A class to store unsigned integer values.

- class column

The class to represent a column of a data table.

- class colValues

A pure virtual base class.

- class compRange

The class compRange stores computed ranges.

- class dictionary

Provide a mapping between strings and integers.

- class direkte

Directly use the integer values as bin number to avoid some intemdiate steps.

- class discretePoisson 
Discrete random number with Poisson distribution $\exp (-x / l a m b d a)$.

- class discretePoisson1

Specialized version of the Poisson distribution $\exp (-x)$.

- class discreteZipf

Discrete Zipf distribution: $p(k)$ is proportional to $(v+k)^{\wedge}(-a)$ where $a>1, k>=0$.

- class discreteZipf1

A specialized case of the Zipf distribution $f(x)=1 /(1+x)$.

- class discreteZipf2

A specialized version of the Zipf distribution $f(x)=1 /(1+x)^{\wedge} 2$.

- class egale

The multicomponent equality code on bins.

- class entre

The multicomponent interval code on bins.

- class fade

The multicomponent range-encoded index.

- class fileManager

This fileManager is intended to allow different objects to share the same open file.

- class fuzz

The two-level interval-equality code.

- class horometer

Horometer - a primitive timing instrument.

- class index

The base index class.

- class keywords

Class ibis: : keywords defines a boolean term-document matrix.

- struct lessi

A case-insensitive version of less for comparing names of tables, columns, and resources.

- class mensa

Class ibis::mensa contains multiple (horizontal) data partitions (ibis::part) to form a logical data table.

- class MersenneTwister

Mersenne Twister generates uniform random numbers efficiently.

- class mesa

This class implements the two-side range encoding from Chan and Ioannidis.

- class meshQuery

The class adds more functionality to ibis::query to handle data from meshes. 
- class moins

The multicomponent range code on bins.

- class nameList

A data structure to store a small set of names.

- class pack

A two-level index.

- class pale

A two-level index.

- class part

The class ibis::part represents a partition of a relational table.

- class qAnyAny

A user specifies this type of query expression with the following syntax,.

- class qContinuousRange

Simple range condition.

- class qDiscreteRange

- class qExpr

The top level query expression object.

- class qMultiString

- class qRange

A class to represent simple range conditions.

- class qString

The class qString encapsulates information for comparing string values.

- class query

A data structure for representing user queries.

- class range

The range encoded bitmap index based.

- class rangeJoin

A join is defined by two names and a numerical expression.

- class relic

The basic bitmap index.

- class resource

A container for name-value pairs.

- union rid_t

The object identifiers used to distinguish records.

- class ridHandler

A class for handling file IO for ibis::rid_t. 
- class roster

A roster list is a list of indices for ordering the values in the ascending order.

- class sapid

The multicomponent equality encoded index.

- class sbiad

The multicomponent interval encoded index.

- class selected

A data structure to store the select clause of a query.

- class slice

The bit-sliced index ( O'Neil). It used the binary encoding.

- class tabele

A trivial class for table with one row and one column.

- class table

The abstract table class.

- class tableList

A list of tables.

- class tablex

The class for expandable tables.

- class tabula

A trivial class for table with no columns.

- class tafel

An expandable table.

- class text

A minimalistic structure for storing arbitrary text fields.

- class uniformRandomNumber

A functor to generate uniform random number in the range $[0,1)$.

- class zona

The two-level equality-equality code.

- class zone

A two-level index.

\section{Typedefs}

- typedef std::vector $<$ colValues $*>$ colList

- typedef std::map $<$ const char $*$, part $*$, lessi $>$ partList

- typedef array_t $<$ rid_t $>$ RIDSet 


\section{Enumerations}

- enum TYPE_T \{

UNKNOWN_TYPE $=0$, OID, BYTE, UBYTE,

SHORT, USHORT, INT, UINT,

LONG, ULONG, FLOAT, DOUBLE,

CATEGORY, TEXT \}

Supported data types.

\section{Functions}

- ibis::resource \& gParameters ()

The reference to the global configuration parameters.

- void init (const int verbose $=0$, const char $*$ rcfile $=0$ )

Initializes internal resources required by ibis.

- const ibis::bitvector64 \& outerProduct (const ibis::bitvector \&a, const ibis::bitvector \&b, ibis::bitvector64 \&c)

Compute the outer product of $\mathrm{a}$ and $\mathrm{b}$, add the result to $\mathrm{C}$.

- const ibis::bitvector64 \& outerProductUpper (const ibis::bitvector \&a, const ibis::bitvector \&b, ibis::bitvector64 \&c)

Add the strict upper triangular portion of the outer production between a and $\mathrm{b}$ to $\mathrm{C}$.

- ibis::qExpr $*$ parseQuery (const char $*$ str)

Parse a query string.

- $\mathrm{qExpr} *$ parseQuery (const char $* \mathrm{str}$ )

Parse a query string.

\section{Variables}

- int accessIndexInWhole $=0$

- int gVerbose

Verbosity level.

- const char $*$ TYPECODE

One-character code for the enumeration types.

- const char $* *$ TYPESTRING

Human readable version of the enumeration types.

\subsubsection{Detailed Description}

The current implementation of FastBit is code named IBIS and most data structures and functions are in the name space ibis.

The name IBIS could be considered as a short-hand for an implementation of Bitmap Index Searching system or Ibis Bitmap Index System. 


\subsubsection{Enumeration Type Documentation}

\subsubsection{1 enum ibis::TYPE_T}

Supported data types.

\section{Enumerator:}

UNKNOWN_TYPE Unknown type, can't really do anything with it.

OID A special eight-byte ID type for internal use.

BYTE One-byte long signed integers.

UBYTE One-byte long unsigned integers.

SHORT Two-byte long signed integers.

USHORT Two-byte long unsigned integers.

INT Four-byte long signed integers.

UINT Four-byte long unsigned integers.

LONG Eight-byte long signed integers.

ULONG Eight-byte long unsigned integers.

FLOAT Four-byte IEEE floating-point numbers.

DOUBLE Eight-byte IEEE floating-point numbers.

CATEGORY Low cardinality null-terminated strings.

TEXT Arbitrary null-terminated strings.

\subsubsection{Function Documentation}

\subsubsection{1 void ibis::init (const int verbose $=0$, const char $* \boldsymbol{r c f i l e}=0) \quad$ [ in l ine ]}

Initializes internal resources required by ibis.

It must be called by user code before any other functions.

\section{Parameters:}

verbose An integer indicating the level of verboseness. A negative number make ibis silent, otherwise the larger it is the more ibis will print out.

rcfile A file containing name-value pairs that specifies parameters for controlling the behavior of ibis. If a file name is not specified, it will attempt to read one of the following file (in the given order).

1. a file named in environment variable IBISRC,

2. a file named ibis.rc in the current working directory,

3. a file named .ibisrc in the user's home directory.

In an $\mathrm{RC}$ file, one parameter occupies a line and the equal sign " $="$ is required to delimit the name and the value, for example,

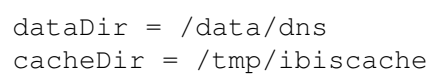

The minimal recommended parameters of an $\mathrm{RC}$ file are

- dataDir, which can also be written as dataDir1 or indexDir. It tells ibis where to find the data to be queried. Multiple data directories may be specified by adding prefix to the parameter name, for example, dns.dataDir and random.dataDir.

- cacheDir, which can also be written as cacheDirectory. This directory is used by ibis to write internal data for recovery and other purposes.

Note:

If this function is not called, the global variables ibis::gParameters must be initialized explicitly before doing anything else. Most useful functions and classes rely on this variable. 
2.1.3.2 const ibis::bitvector64 \& ibis::outerProduct (const ibis::bitvector \& $a$, const ibis::bitvector \& $b$, ibis::bitvector64 \& $c$ )

Compute the outer product of $a$ and $b$, add the result to $c$.

This should make it possible to use both WAH and BBC compress bitvector classes. It is not likely that we can gain much performance by directly using member variables of ibit::bitvector because the unit of compressed data from ibit::vector do not fit neatly into ibis::bitvector64::word_t.

\subsubsection{3 ibis::qExpr $*$ ibis::parseQuery (const char $*$ str)}

Parse a query string.

The query expression is stored in a binary tree (ibis::qExpr). There is a mutual exclusion lock to serialize accesses to this function. The implication is that only one query can be parsed at any given time.

\subsubsection{4 qExpr $*$ ibis::parseQuery (const char $*$ str)}

Parse a query string.

The query expression is stored in a binary tree (ibis::qExpr). There is a mutual exclusion lock to serialize accesses to this function. The implication is that only one query can be parsed at any given time.

\subsubsection{Variable Documentation}

\subsubsection{1 int ibis::gVerbose}

Verbosity level.

The larger the value, the more is printed. Default value is 0 . A negative value will disable all printing.

\section{2 std Namespace Reference}

STL namespace.

\section{Classes}

- class allocator

STL class.

- class auto_ptr

STL class.

- class bad_alloc

STL class.

- class bad_cast

STL class.

- class bad_exception

STL class.

- class bad_typeid

STL class.

- class basic_fstream 
STL class.

- class basic_ifstream

STL class.

- class basic_ios

STL class.

- class basic_iostream

STL class.

- class basic_istream

STL class.

- class basic_istringstream

STL class.

- class basic_ofstream

STL class.

- class basic_ostream

STL class.

- class basic_ostringstream

STL class.

- class basic_string

STL class.

- class basic_stringstream

STL class.

- class bitset

STL class.

- class complex

STL class.

- class deque

STL class.

- class domain_error

STL class.

- class exception

STL class.

- class fstream

STL class.

- class ifstream

STL class. 
- class invalid_argument STL class.

- class ios STL class.

- class ios_base STL class.

- class istream STL class.

- class istringstream STL class.

- class length_error STL class.

- struct less $<$ char $*>$

- struct less $<$ const char $*>$

- struct less $<$ const ibis::rid_t $*>$

- struct less $<$ ibis::rid_t $>$

- class list

STL class.

- class logic_error STL class.

- class map STL class.

- class multimap STL class.

- class multiset

$$
\text { STL class. }
$$

- class ofstream

$$
\text { STL class. }
$$

- class ostream STL class.

- class ostringstream STL class.

- class out_of_range

$$
\text { STL class. }
$$

- class overflow_error STL class.

- class priority_queue 
STL class.

- class queue

STL class.

- class range_error

STL class.

- class runtime_error

STL class.

- class set

STL class.

- class stack

STL class.

- class string

STL class.

- class stringstream

STL class.

- class underflow_error

STL class.

- class valarray

STL class.

- class vector

STL class.

- class wfstream

STL class.

- class wifstream

STL class.

- class wios

STL class.

- class wistream

STL class.

- class wistringstream

STL class.

- class wofstream

STL class.

- class wostream

STL class. 
- class wostringstream

STL class.

- class wstring

STL class.

- class wstringstream

STL class.

\subsubsection{Detailed Description}

STL namespace.

\section{FastBit Class Documentation}

\section{1 array_t $<\mathbf{T}>$ Class Template Reference}

Template array_t implements a replacement of std::vector.

\#include <array_t.h>

\section{Public Types}

- typedef const $\mathrm{T} *$ const_iterator

- typedef $\mathrm{T} *$ iterator

\section{Public Member Functions}

- array_t (const char $* \mathrm{fn}$, const off_t begin, const off_t end)

Retrieve a portion of the named file to an array.

- array_t (const int fdes, const off_t begin, const off_t end)

Read a portion of an open file into an array.

- array_t (ibis::fileManager::storage $*$ rhs, const uint32_t start, const uint32_t nelm)

Construct an array from a section of the raw storage.

- array_t (ibis::fileManager::storage \&rhs)

Turn a raw storage object into an array_t object.

- array_t (const array_t $<\mathrm{T}>$ \&rhs, const uint32_t offset, const uint32_t nelm=0)

A shallow copy constructor.

- array_t (const array_t $<\mathrm{T}>$ \&rhs)

Shallow copy. Should not throw any exception.

- array_t (uint32_t n, const T \&val)

Construct an array with $\mathrm{n}$ elements of value val.

- array_t (uint32_t n) 
Construct an array with n elements.

- $\operatorname{array\_ t}()$

The default constructor. It constructs an empty array.

- const T \& back () const

- $\mathrm{T} \&$ back ()

- const $\mathrm{T} *$ begin () const

- $\mathrm{T} * \operatorname{begin}()$

- void bottomk (uint32_t k, array_t $<$ uint32_t $>$ \&ind) const

Return the positions of the $\mathrm{k}$ smallest elements.

- void clear ()

- void copy (const array_t $<\mathrm{T}>$ \&rhs)

The copy function. It performs a shallow copy.

- $\operatorname{void}$ deepCopy (const array_t $\mathrm{t}<\mathrm{T}>$ \&rhs)

The deep copy function.

- int empty () const

- const $\mathrm{T} *$ end () const

- $\mathrm{T} *$ end ()

- iterator erase (iterator i, iterator $\mathrm{j}$ )

- iterator erase (iterator $\mathrm{p}$ )

- uint32_t find (const T \&val) const

Return the smallest $i$ such that $[i]>=$ val.

- uint32_t find (const array_t $<$ uint32_t $>$ \&ind, const T \&val) const

Return the smallest $i$ such that [ind $[i]]>=$ val.

- const T \& front () const

- T \& front ()

- void insert (iterator p, const_iterator i, const_iterator j)

- void insert (iterator p, uint32_t n, const T \&val)

- iterator insert (iterator pos, const T \&val)

Insert one value or a list of values before $p$.

- void nosharing ()

Make a not-shared copy of the array if it is actually a shared array.

- const array_t $<\mathrm{T}>\&$ operator= (const array_t $\mathrm{t}<\mathrm{T}>$ \&rhs)

Assignment operator. It performs a shallow copy.

- T \& operator[] (uint32_t i)

Modifiable reference to an element of the array.

- const T \& operator[ ] (uint32_t i) const

Non-modifiable reference to an element of the array.

- void pop_back ()

Reset the size to zero.

- void printStatus (std::ostream \&out) const 
Print internal pointer addresses.

- void push_back (const T \&elm)

Add one element.

- uint32_t read (const int fdes, const uint32_t begin, const uint32_t end)

Read a portion of an open file.

- $\operatorname{void}$ read (const char $*$ )

Read an array from the name file.

- void reserve (uint32_t n)

Reserve space.

- void resize (uint32_t $\mathrm{n}$ )

Remove the last element. Resize array.

- uint32_t size () const

- void sort (array_t $<$ uint32_t $>$ \&ind) const

Produce index for ascending order.

- void stableSort (array_t $<$ uint32_t $>$ \&ind, array_t $<\mathrm{T}>$ \& sorted) const

A stable sort.

- void stableSort (array_t $<$ uint32_t $>$ \&ind) const

A stable sort that does not modify the current array.

- void stableSort (array_t $<\mathrm{T}>\& \mathrm{tmp}$ )

A stable sort using the provided workspace.

- void swap (array_t $\mathrm{t}<\mathrm{T}>$ \&rhs)

Exchange the content.

- void topk (uint32_t $\mathrm{k}$, array_t $<$ uint32_t $>$ \&ind) const

Return the positions of the $\mathrm{k}$ largest elements.

- void write (FILE $*$ fptr) const

write whole array to an opened file

- void write (const char $*$ ) const

write whole array to the named file

\section{Static Public Member Functions}

- static void stableSort (array_t $<\mathrm{T}>\&$ val, array_t $<$ uint32_t $>$ \&ind, array_t $<\mathrm{T}>$ \& tmp, array_t $<$ uint32_t $>$ \&itmp)

This function sorts the content of array val. 


\subsubsection{Detailed Description}

template $<$ class $\mathbf{T}>$ class array_t $<\mathbf{T}>$

Template array_t implements a replacement of std::vector.

The main difference is that the underlying memory may be managed by ibis::fileManager. In addition, it also implements read and write functions that are not present in std::vector.

\subsubsection{Constructor \& Destructor Documentation}

3.1.2.1 template $<$ class $\mathbf{T}>$ array_t $<\mathbf{T}>$ ::array_t (const array_t $<\mathbf{T}>\boldsymbol{\&} r h s$, const uint32_t offset, const uint32_t nelm $=0$ )

A shallow copy constructor.

It makes a new array out of a section of an existing array.

\subsubsection{2 template $<$ class $T>$ array_t $<\mathbf{T}>$ ::array_t (ibis::fileManager::storage $\&$ rhs )}

Turn a raw storage object into an array_t object.

The input storage object is used by the array. No new storage is allocated.

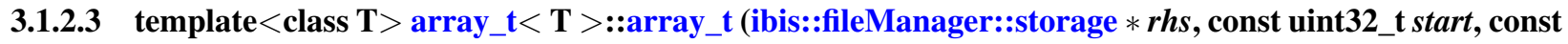
uint32_t nelm)

Construct an array from a section of the raw storage.

The argument start is measured in number of bytes, the second argument nelm is the number of element of type T.

\subsubsection{4 template $<$ class $T>$ array_t $<T$ T : array_t (const int fdes, const off_t begin, const off_t end)}

Read a portion of an open file into an array.

The argument fdes must be a valid file descriptor (as defined in unistd.h). It attempt to read end - begin bytes from the file starting at offset begin.

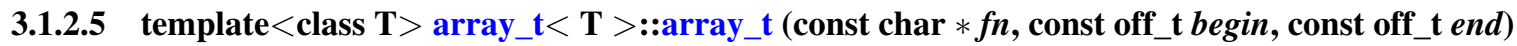

Retrieve a portion of the named file to an array.

Prefer memory map if possible.

\subsubsection{Member Function Documentation}

\subsubsection{1 template $<$ class $\mathbf{T}>$ void array_t $<T$ T::bottomk (uint32_t $k$, array_t $<$ uint32_t $>\&$ ind) const}

Return the positions of the $\mathrm{k}$ smallest elements.

Return the indices of the smallest values in array ind.

Note:

The resulting array ind may have more than $k$ elements if the $k$ th smallest value is not a single value. The array ind may have less than $\mathrm{k}$ elements if this array has less than $\mathrm{k}$ elements. 


\subsubsection{2 template $<$ class $\mathbf{T}>$ void array_t $<\mathbf{T}>$ ::deepCopy (const array_t $<\mathbf{T}>\boldsymbol{\&}$ rhs )}

The deep copy function.

It makes an in-memory version of the array rhs.

\subsubsection{3 template $<$ class $T>$ uint32_t array_t $<$ T $>$ ::find (const $T \&$ val) const}

Return the smallest i such that $[\mathrm{i}]>=$ val.

Assume the array is sorted in ascending order.

\subsubsection{4 template $<$ class $T>$ uint32_t array_t $<T>$ ::find (const array_t $<$ uint32_t $>\&$ ind, const $T \&$ val)} const

Return the smallest i such that [ind[i]] $>=$ val.

Assuming ind was produced by the sort function, it returns the smallest i such that operator [ ] (ind[i]) $>=$ val.

\subsubsection{5 template $<$ class $\mathbf{T}>$ iterator array_t $<$ T $>$ ::insert (iterator pos, const $\mathbf{T} \& \mathrm{val}$ )}

Insert one value or a list of values before $p$.

Return pointer to new elem.

\subsubsection{6 template $<$ class $\mathbf{T}>$ void array_t $<\mathbf{T}>$ ::nosharing ()}

Make a not-shared copy of the array if it is actually a shared array.

This does not guarantee that it would not become shared later. The complete solution is to implement copy-on-write in all functions that modifies an array, but that may decrease performance of this class for rare cases of modifications.

\subsubsection{7 ] template $<$ class $\mathrm{T}>\mathrm{T} \&$ array_t $<\mathrm{T}>$ ::operator[] (uint32_t $i$ ) [inline ]}

Modifiable reference to an element of the array.

Note:

For efficiency reasons, this is not a copy-on-write implementation! The caller has to call the function no sharing to make sure the underlying data is not shared with others.

\subsubsection{8 template $<$ class $T>$ void array_t $<T>$ ::reserve (uint32_t $n$ )}

Reserve space.

If the current storage object does not have enough space, enlarge the storage object.

\subsubsection{9 template $<$ class $\mathbf{T}>$ uint32_t array_t $<\mathbf{T}>$ ::size () const $[$ in line ]}

3.1.3.10 template $<$ class $\mathbf{T}>$ void array_t $<\mathbf{T}>$ : :stableSort $\left(\operatorname{array} \_t<T>\&\right.$ val, array_t $<$ uint32_t $>$ \& ind, array_t $<\mathbf{T}>\boldsymbol{\&} \mathbf{t m p}$, array_t $\mathbf{t}<\mathbf{u i n t 3 2} \mathbf{t}>\boldsymbol{\&}$ itmp) [ static ]

This function sorts the content of array val.

The values of ind[i] will be reordered in the same way as the array val. The two other arrays are used as temporary storage. 


\section{Note:}

On input, if array ind has the same size as array val, the content of array ind will be directly used. Otherwise, array ind will be initialized to be consecutive integers starting from 0 .

If the input array val has less than two elements, this function does nothing, i.e., does not change any of the four arguments.

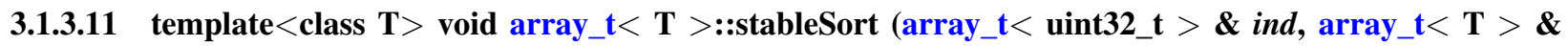
sorted) const

A stable sort.

It does not change this array, but produces a sorted version in sorted.

\subsubsection{2 template $<$ class $\mathbf{T}>$ void array_t $<\mathbf{T}>$ : :stableSort $($ array_t $<$ uint32_t $>\&$ ind) const}

A stable sort that does not modify the current array.

It uses two additional arrays for temporary storage.

\subsubsection{3 template $<$ class $\mathbf{T}>$ void array_t $<\mathbf{T}>$ ::stableSort $\left(\operatorname{array\_ } \mathbf{t}<\mathbf{T}>\boldsymbol{\&}\right.$ tmp $)$}

A stable sort using the provided workspace.

The current content is modified to be in ascending order. The argument tmp is only used as temporary storage.

\subsubsection{4 template $<$ class $T>$ void array_t $<T>$ ::topk (uint32_t $k$, array_t $<$ uint32_t $>\&$ ind) const}

Return the positions of the $\mathrm{k}$ largest elements.

Return the indices of the in sorted values.

Note:

The resulting array ind may have more than $\mathrm{k}$ elements if the $\mathrm{k}$ th smallest value is not a single value. The array ind may have less than $\mathrm{k}$ elements if this array has less than $\mathrm{k}$ elements.

The values are sorted in ascending order, i.e., [ind [i] ] $<=[i n d[i+1]]$. This is done so that all sorting routines produce indices in the same ascending order. It should be easy to reverse the order the indices since it only contains the largest values.

The documentation for this class was generated from the following files:

- array_t.h

- array_t.cpp

\section{2 ibis::ambit Class Reference}

The multi-level range based (cumulative) index.

\#include <ibin.h>

Inheritance diagram for ibis::ambit::

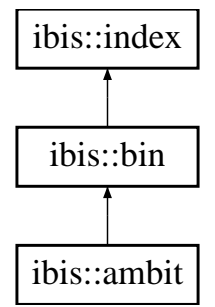




\section{Public Member Functions}

- virtual void adjustLength (uint32_t nrows)

- ambit (const ibis::bin \&rhs)

- ambit (const ibis::column $*$ c, ibis::fileManager::storage $*$ st, uint32_t offset=8)

- ambit (const ibis::column $* \mathrm{c}=0$, const char $* \mathrm{f}=0$ )

- long append (const ibis::ambit \&tail)

- virtual long append (const char $* \mathrm{dt}$, const char $* \mathrm{df}$, uint32_t nnew)

Extend the index.

- virtual void binBoundaries (std::vector $<$ double $>\&$ ) const

The function binBoundaries and binWeights return bin boundaries and counts of each bin respectively.

- virtual void binWeights (std::vector $<$ uint32_t $>$ \&) const

- virtual void estimate (const ibis::qContinuousRange \&expr, ibis::bitvector \&lower, ibis::bitvector \&upper) const

Computes an approximation of hits as a pair of lower and upper bounds.

- virtual long evaluate (const ibis::qContinuousRange \&expr, ibis::bitvector \&hits) const

To evaluate the exact hits.

- virtual double getSum () const

Compute the approximate sum of all the values indexed.

- virtual const char $*$ name () const

Returns the name of the index, similar to the function type, but returns a string instead.

- virtual uint32_t numBins () const

- virtual void print (std::ostream \&out) const

Prints human readable information.

- virtual void read (ibis::fileManager::storage $*$ st)

Reconstructs an index from an array of bytes.

- virtual void read (const char $*$ idxfile)

Reconstructs an index from the named file.

- virtual void speedTest (std::ostream \&out) const

Time some logical operations and print out their speed.

- virtual INDEX_TYPE type () const

Returns an index type identifier.

- virtual float undecidable (const ibis::qContinuousRange \&expr, ibis::bitvector \&iffy) const

Mark the position of the rows that can not be decided with this index.

- virtual void write (const char $* \mathrm{dt}$ ) const

Save index to a file.

\section{Protected Member Functions}

- virtual double computeSum () const 


\subsubsection{Detailed Description}

The multi-level range based (cumulative) index.

Each level/each bin consists of a range index.

\subsubsection{Member Function Documentation}

3.2.2.1 void ibis::ambit::estimate (const ibis::qContinuousRange \& expr, ibis::bitvector \& lower, ibis::bitvector \& upper) const [virtual]

Computes an approximation of hits as a pair of lower and upper bounds.

\section{Parameters:}

expr the query expression to be evaluated.

lower a bitvector marking a subset of the hits. All rows marked with one (1) are definitely hits.

upper a bitvector marking a superset of the hits. All hits are marked with one, but some of the rows marked one may not be hits. If the variable upper is empty, the variable lower is assumed to contain the exact answer.

Reimplemented from ibis::bin.

3.2.2.2 long ibis::ambit::evaluate (const ibis::qContinuousRange \& expr, ibis::bitvector \& hits) const [virtual]

To evaluate the exact hits.

On success, return the number of hits, otherwise a negative value is returned.

Reimplemented from ibis::bin.

\subsubsection{3 double ibis::ambit::getSum () const [virtual]}

Compute the approximate sum of all the values indexed.

If it decides that computing the sum directly from the vertical partition is more efficient, it will return NaN immediately. Reimplemented from ibis::bin.

\subsubsection{4 void ibis::ambit::print (std::ostream \& out) const [virtual]}

Prints human readable information.

Outputs information about the index as text to the specified output stream.

Reimplemented from ibis::bin.

\subsubsection{5 void ibis::ambit::read (ibis::fileManager::storage $* s t$ ) [virtual]}

Reconstructs an index from an array of bytes.

Intended for internal use only!

Reimplemented from ibis::bin.

\subsubsection{6 void ibis::ambit::read (const char $*$ idxfile) [virtual]}

Reconstructs an index from the named file.

The name can be the directory containing an index file. In this case, the name of the index file must be the name of the column followed by ".idx" suffix.

Reimplemented from ibis::bin. 
3.2.2.7 float ibis::ambit::undecidable (const ibis::qContinuousRange \& expr, ibis::bitvector \& iffy) const [virtual]

Mark the position of the rows that can not be decided with this index.

\section{Parameters:}

expr the range conditions to be evaluated.

iffy the bitvector marking the positions of rows that can not be decided using the index. Return value is the expected fraction of undecided rows that might satisfy the range conditions.

Reimplemented from ibis::bin.

\subsubsection{8 void ibis::ambit::write (const char $* \boldsymbol{d} \boldsymbol{t})$ const [virtual]}

Save index to a file.

Outputs the index in a compact binary format to the named file or directory. The index file contains a header that can be identified by the function isIndex.

Reimplemented from ibis::bin.

The documentation for this class was generated from the following files:

- ibin.h

- ixambit.cpp

\section{3 ibis::bad_alloc Class Reference}

A specialization of std::bad_alloc.

\#include <util.h>

Inheritance diagram for ibis::bad_alloc::

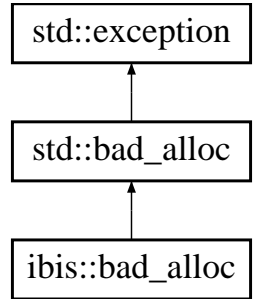

\section{Public Member Functions}

- bad_alloc (const char $*$ m="unknown") throw ()

- virtual const char $*$ what () const throw ()

\subsubsection{Detailed Description}

A specialization of std::bad_alloc.

The documentation for this class was generated from the following file:

- util.h 


\section{4 ibis::bak Class Reference}

Maps each value to a lower prevision (decimal) values and use the the low precision value as center of the bin.

\#include <ibin.h>

Inheritance diagram for ibis::bak::

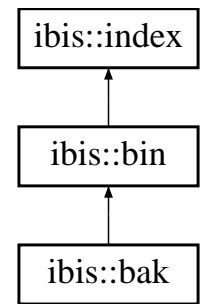

\section{Public Types}

- typedef std::map $<$ double, grain $>$ bakMap

\section{Public Member Functions}

- long append (const ibis::bin \&tail)

Append the tail to this index.

- virtual long append (const char $* \mathrm{dt}$, const char $* \mathrm{df}$, uint32_t nnew)

Extend the index.

- bak (const ibis::column $*$ c, ibis::fileManager::storage $*$ st, uint32_t offset=8)

- bak (const ibis::column $* \mathrm{c}=0$, const char $* \mathrm{f}=0$ )

- virtual void binBoundaries (std::vector $<$ double $>\&$ ) const

The function binBoundaries and binWeights return bin boundaries and counts of each bin respectively.

- virtual void binWeights (std::vector $<$ uint32_t $>\&$ ) const

- virtual int contractRange (ibis::qContinuousRange \&rng) const

- virtual int expandRange (ibis::qContinuousRange \&rng) const

The functions expandRange and contractRange expands or contracts the boundaries of a range condition so that the new range will have exact answers using the function estimate.

- virtual const char $*$ name () const

Returns the name of the index, similar to the function type, but returns a string instead.

- virtual void print (std::ostream \&out) const

Prints human readable information.

- virtual void read (const char $*$ idxfile)

Reconstructs an index from the named file.

- virtual INDEX_TYPE type () const

Returns an index type identifier.

- virtual void write (const char $* \mathrm{dt}$ ) const

Save index to a file. 


\section{Protected Member Functions}

- virtual void locate (const ibis::qContinuousRange \&expr, uint32_t \&cand0, uint32_t \&cand1, uint32_t \&hit0, uint32_t \&hit1) const

- virtual void locate (const ibis::qContinuousRange \&expr, uint32_t \&cand0, uint32_t \&cand1) const

- virtual uint32_t locate (const double \&val) const

- void mapValues (const char $* \mathrm{f}$, bakMap \&bmap) const

- void printMap (std::ostream \&out, const bakMap \&bmap) const

\section{Classes}

- struct grain

\subsubsection{Detailed Description}

Maps each value to a lower prevision (decimal) values and use the the low precision value as center of the bin.

It reuses the same variables of ibis::bin, but have to interpret them differently.

Bak is a Dutch word for 'bin'.

\subsubsection{Member Function Documentation}

\subsubsection{1 int ibis::bak::expandRange (ibis::qContinuousRange \& $\boldsymbol{r n g}$ ) const [virtual]}

The functions expandRange and contractRange expands or contracts the boundaries of a range condition so that the new range will have exact answers using the function estimate.

The default implementation provided does nothing since this is only meaningful for indices based on bins.

Reimplemented from ibis::bin.

\subsubsection{2 void ibis::bak::print (std::ostream \& out) const [ virtual]}

Prints human readable information.

Outputs information about the index as text to the specified output stream.

Reimplemented from ibis::bin.

\subsubsection{3 void ibis::bak::read (const char $*$ idxfile) $\quad$ [virtual]}

Reconstructs an index from the named file.

The name can be the directory containing an index file. In this case, the name of the index file must be the name of the column followed by ".idx" suffix.

Reimplemented from ibis::bin.

\subsubsection{4 void ibis::bak::write (const char $* \boldsymbol{d t}$ ) const [virtual]}

Save index to a file.

Outputs the index in a compact binary format to the named file or directory. The index file contains a header that can be identified by the function isIndex.

Reimplemented from ibis::bin.

The documentation for this class was generated from the following files:

- ibin.h 
- idbak.cpp

\section{5 ibis::bak2 Class Reference}

A variation on ibis: : bak, it splits each bin of ibis::bak in two, one for entries less than the mapped value and one for the entries that greater and equal to the mapped value.

\#include <ibin.h>

Inheritance diagram for ibis::bak2::

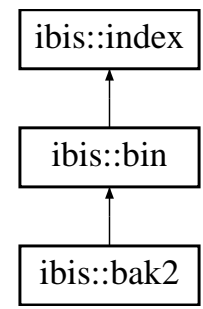

\section{Public Types}

- typedef std::map $<$ double, grain $>$ bakMap

\section{Public Member Functions}

- long append (const ibis::bin \&tail)

Append the tail to this index.

- virtual long append (const char $* \mathrm{dt}$, const char $* \mathrm{df}$, uint32_t nnew)

Extend the index.

- bak2 (const ibis::column $*$ c, ibis::fileManager::storage $*$ st, uint32_t offset=8)

- bak2 (const ibis::column $* \mathrm{c}=0$, const char $* \mathrm{f}=0$ )

- virtual void binBoundaries (std::vector $<$ double $>$ \&) const

The function binBoundaries and binWeights return bin boundaries and counts of each bin respectively.

- virtual void binWeights (std::vector $<$ uint32_t $>\&$ ) const

- virtual int contractRange (ibis::qContinuousRange \&rng) const

- virtual int expandRange (ibis::qContinuousRange \&rng) const

The functions expandRange and contractRange expands or contracts the boundaries of a range condition so that the new range will have exact answers using the function estimate.

- virtual const char $*$ name () const

Returns the name of the index, similar to the function type, but returns a string instead.

- virtual void print (std::ostream \&out) const

Prints human readable information.

- virtual void read (const char $*$ idxfile)

Reconstructs an index from the named file.

- virtual INDEX_TYPE type () const

Returns an index type identifier. 
- virtual void write (const char $* \mathrm{dt}$ ) const Save index to a file.

\section{Protected Member Functions}

- virtual void locate (const ibis::qContinuousRange \&expr, uint32_t \&cand0, uint32_t \&cand1, uint32_t \&hit0, uint32_t \&hit1) const

- virtual void locate (const ibis::qContinuousRange \&expr, uint32_t \&cand0, uint32_t \&cand1) const

- virtual uint32_t locate (const double \&val) const

- void mapValues (const char $*$ f, bakMap \&bmap) const

Reads all values and records positions in bmap.

- void printMap (std::ostream \&out, const bakMap \&bmap) const

\section{Classes}

- struct grain

A simple structure to record the position of the values mapped to the same value.

\subsubsection{Detailed Description}

A variation on ibis: : bak, it splits each bin of ibis::bak in two, one for entries less than the mapped value and one for the entries that greater and equal to the mapped value.

This way, the index can be used to answer question involving ranges exactly on the mapped values. All internal variables are processed same as a regular ibis::bin index.

\subsubsection{Member Function Documentation}

\subsubsection{1 int ibis::bak2::expandRange (ibis::qContinuousRange \& rng) const [virtual]}

The functions expandRange and contractRange expands or contracts the boundaries of a range condition so that the new range will have exact answers using the function estimate.

The default implementation provided does nothing since this is only meaningful for indices based on bins.

Reimplemented from ibis::bin.

\subsubsection{2 void ibis::bak2::print (std::ostream \& out) const [ virtual]}

Prints human readable information.

Outputs information about the index as text to the specified output stream.

Reimplemented from ibis::bin.

\subsubsection{3 void ibis::bak2::read (const char $*$ idxfile) $\quad$ [virtual]}

Reconstructs an index from the named file.

The name can be the directory containing an index file. In this case, the name of the index file must be the name of the column followed by ".idx" suffix.

Reimplemented from ibis::bin. 


\subsubsection{4 void ibis::bak2::write (const char $* \boldsymbol{d t}$ ) const [virtual]}

Save index to a file.

Outputs the index in a compact binary format to the named file or directory. The index file contains a header that can be identified by the function isIndex.

Reimplemented from ibis::bin.

The documentation for this class was generated from the following files:

- ibin.h

- idbak2.cpp

\section{6 ibis::bak2::grain Struct Reference}

A simple structure to record the position of the values mapped to the same value.

\#include <ibin.h>

\section{Public Attributes}

- ibis::bitvector $*$ loc0

- ibis::bitvector $*$ loc1

- double max0

- double max1

- double min0

- double min1

\subsubsection{Detailed Description}

A simple structure to record the position of the values mapped to the same value.

The ibis: :bitvector marked the locations of the values and the min and max record the actual minimum and maximum value encountered.

The documentation for this struct was generated from the following file:

- ibin.h

\section{7 ibis::bin Class Reference}

The equality encoded bitmap index with binning.

\#include <ibin.h>

Inheritance diagram for ibis::bin:: 


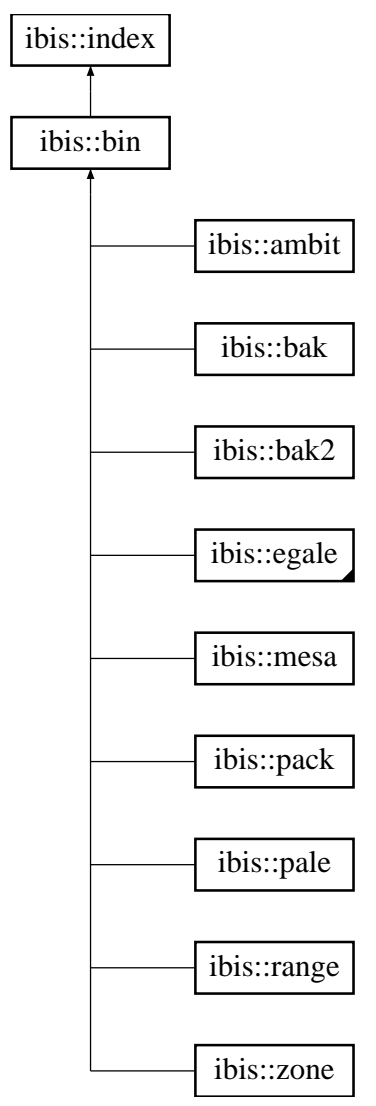

\section{Public Types}

- typedef std::map $<$ double, granule $*>$ granuleMap

\section{Public Member Functions}

- long append (const array_t $<$ uint32_t $>$ \&ind)

Append a list of integers representing bin numbers.

- long append (const ibis::bin \&tail)

Append the tail to this index.

- virtual long append (const char $* \mathrm{dt}$, const char $* \mathrm{df}$, uint32_t nnew)

Extend the index.

- bin (const ibis::column $*$ c, const char $*$ f, const std::vector $<$ double $>\&$ bd)

- bin (const ibis:: column $*$ c, const char $*$ f, const array_t $\mathrm{t}<$ double $>\& b d$ )

- bin (const ibis::column $*$ c, ibis::fileManager::storage $*$ st, uint32_t offset=8)

- bin (const ibis:: column $* \mathrm{c}=0$, const char $* \mathrm{f}=0$ )

Construct a bitmap index from current data.

- bin (const ibis::bin \&rhs)

- virtual void binBoundaries (std::vector $<$ double $>\&$ ) const

The function binBoundaries and binWeights return bin boundaries and counts of each bin respectively.

- virtual void binWeights (std::vector $<$ uint32_t $>\&$ ) const 
- long checkBin (const ibis::qRange \&cmp, uint32_t jbin, const ibis::bitvector \&mask, ibis::bitvector \&res) const

Candidate check using the binned values.

- long checkBin (const ibis::qRange \&cmp, uint32_t jbin, ibis::bitvector \&res) const

Candidate check using the binned values.

- virtual int contractRange (ibis::qContinuousRange \&rng) const

- virtual int64_t estimate (const ibis::bin \&idx2, const ibis::rangeJoin \&expr, const ibis::bitvector \&mask, const ibis::qRange $*$ const range1, const ibis::qRange $*$ const range2) const

- virtual int64_t estimate (const ibis::bin \&idx2, const ibis::rangeJoin \&expr, const ibis::bitvector \&mask) const

- virtual int64_t estimate (const ibis::bin \&idx2, const ibis::rangeJoin \&expr) const

- virtual void estimate (const ibis::bin \&idx2, const ibis::rangeJoin \&expr, const ibis::bitvector \&mask, const ibis::qRange $*$ const range1, const ibis::qRange $*$ const range2, ibis::bitvector64 \&lower, ibis::bitvector64 \&upper) const

- virtual void estimate (const ibis::bin \&idx2, const ibis::rangeJoin \&expr, const ibis::bitvector \&mask, ibis::bitvector64 \&lower, ibis::bitvector64 \&upper) const

- virtual void estimate (const ibis::bin \&idx2, const ibis::rangeJoin \&expr, ibis::bitvector64 \&lower, ibis::bitvector64 \&upper) const

Estimate the number of hits for nonsymmetric joins.

- virtual int64_t estimate (const ibis::rangeJoin \&expr, const ibis::bitvector \&mask, const ibis::qRange $*$ const range 1 , const ibis::qRange $*$ const range2) const

- virtual void estimate (const ibis::rangeJoin \&expr, const ibis::bitvector \&mask, const ibis::qRange $*$ const range1, const ibis::qRange $*$ const range2, ibis::bitvector64 \&lower, ibis::bitvector64 \&upper) const

Evaluating a join condition with one (likely composite) index.

- virtual void estimate (const ibis::rangeJoin \&expr, const ibis::bitvector \&mask, ibis::bitvector64 \&lower, ibis::bitvector64 \&upper) const

- virtual void estimate (const ibis::rangeJoin \&expr, ibis::bitvector64 \&lower, ibis::bitvector64 \&upper) const

Estimate the hits for symmetric joins.

- virtual uint32_t estimate (const ibis::qContinuousRange \&expr) const

Returns an upper bound on the number of hits.

- virtual void estimate (const ibis::qContinuousRange \&expr, ibis::bitvector \&lower, ibis::bitvector \&upper) const

Computes an approximation of hits as a pair of lower and upper bounds.

- virtual double estimateCost (const ibis::qDiscreteRange \&expr) const

- virtual double estimateCost (const ibis::qContinuousRange \&expr) const

Estimate the code of evaluate a range condition.

- virtual long evaluate (const ibis::qContinuousRange \&expr, ibis::bitvector \&hits) const

To evaluate the exact hits.

- virtual int expandRange (ibis::qContinuousRange \&rng) const

The functions expandRange and contractRange expands or contracts the boundaries of a range condition so that the new range will have exact answers using the function estimate.

- virtual long getCumulativeDistribution (std::vector $<$ double $>$ \&bds, std::vector $<$ uint32_t $>$ \&cts) const

Cumulative distribution of the data.

- virtual long getDistribution (std::vector $<$ double $>\&$ bbs, std::vector $<$ uint32_t $>\&$ cts) const 
Binned distribution of the data.

- virtual double getMax () const

The maximum value recorded in the index.

- virtual double getMin () const

The minimum value recorded in the index.

- virtual double getSum () const

Compute the approximate sum of all the values indexed.

- $\operatorname{array} \_\mathrm{t}<$ uint32_t $>*$ indices (const ibis::bitvector \&mask) const

- virtual const char $*$ name () const

Returns the name of the index, similar to the function type, but returns a string instead.

- virtual uint32_t numBins () const

- virtual void print (std::ostream \&out) const

Prints human readable information.

- void read (int fdes, uint32_t offset, const char $*$ fname)

Read an ibis:: bin embedded inside a file.

- virtual void read (ibis::fileManager::storage $*$ st)

Reconstructs an index from an array of bytes.

- virtual void read (const char $*$ idxfile)

Reconstructs an index from the named file.

- virtual void speedTest (std::ostream \&out) const

Time some logical operations and print out their speed.

- virtual INDEX_TYPE type () const

Returns an index type identifier.

- virtual float undecidable (const ibis::qContinuousRange \&expr, ibis::bitvector \&iffy) const

Mark the position of the rows that can not be decided with this index.

- virtual void write (const char $* \mathrm{dt}$ ) const

Save index to a file.

\section{Protected Member Functions}

- void addBounds (double lbd, double rbd, uint32_t nbins, uint32_t eqw)

- virtual void adjustLength (uint32_t nrows)

- bin (const ibis::column $*$ c, const uint32_t nbits, ibis::fileManager::storage $*$ st, uint32_t offset=8)

A constructor to handle the common portion of multicomponent encodings.

- template $<$ typename $\mathrm{E}>$ void binning (const array_t $<\mathrm{E}>$ \& varr, const array_t $<$ double $>$ \&bd)

- template $<$ typename $\mathrm{E}>$ void binning (const array_t $<\mathrm{E}>$ \& varr)

- void binning (const char $* \mathrm{f}$ )

Read the data file and partition the values into bins according to the specified bin boundary. 
- $\operatorname{void}$ binning (const char $* \mathrm{f}$, const array_t $\mathrm{t}<$ double $>$ \&bd)

- void binning (const char $*$ f, const std::vector $<$ double $>\& b d$ )

Generate bins according to the specified boundaries.

- template $<$ typename $\mathrm{E}>$ void binningT (const char $*$ fname)

Read the data file, partition the values, and write out the bin ordered data with bin suffix.

- long binOrder (const char $*$ fname) const

- template $<$ typename $\mathrm{E}>$ long binOrderT (const char $*$ fname) const

Write bin-ordered values.

- template $<$ typename $\mathrm{E}>$ long checkBin0 (const ibis::qRange \&cmp, uint32_t jbin, ibis::bitvector \&res) const

- template $<$ typename $\mathrm{E}>$ long checkBin1 (const ibis::qRange \&cmp, uint32_t jbin, const ibis::bitvector \&mask, ibis::bitvector \&res) const

- virtual void clear ()

Clear the existing content.

- virtual double computeSum () const

- template $<$ typename $\mathrm{E}>$ void construct (const array_t $<\mathrm{E}>$ \&varr)

- void convertGranules (granuleMap \& gmap)

- void divideBitmaps (const std::vector $<$ ibis::bitvector $*>$ \&bms, std::vector $<$ unsigned $>$ \&parts) const

- virtual void locate (const ibis::qContinuousRange \&expr, uint32_t \&cand0, uint32_t \&cand1, uint32_t \&hit0, uint32_t \&hit1) const

- virtual void locate (const ibis::qContinuousRange \&expr, uint32_t \&cand0, uint32_t \&cand1) const

- virtual uint32_t locate (const double \&val) const

- template $<$ typename $\mathrm{E}>$ void mapGranules (const array_t $<\mathrm{E}>$ \&, granuleMap \&gmap) const

- uint32_t parseNbins () const

- unsigned parsePrec () const

- unsigned parseScale () const

- void printGranules (std::ostream \&out, const granuleMap \&gmap) const

- void readBinBoundaries (const char $*$ name, uint32_t nb)

- void scanAndPartition (const char $*$, unsigned, uint32_t nbins=0)

The optional argument nbins can either be set outside or set to be the return value of function parseNbins.

- template $<$ typename $\mathrm{E}>$ void scanAndPartition (const array_t $<\mathrm{E}>$ \&, unsigned)

- template $<$ typename $\mathrm{E}>$ void setBoundaries (const array_t $<\mathrm{E}>$ \&varr)

- void setBoundaries (array_t $<$ double $>$ \&bnds, const ibis::bin \&idx 1 , const array_ $t<$ uint32_t $>$ cnt 1 , const array_t $<$ uint32_t $>$ cnt0) const

- void setBoundaries (array_t $<$ double $>\&$ bnds, const ibis::bin \&bin0) const

- void setBoundaries (const char $* \mathrm{f}$ )

Set bin boundaries.

- void swap (bin \&rhs)

\section{Protected Attributes}

- array_ $\mathrm{t}<$ double $>$ bounds

The nominal boundaries.

- array_t $<$ double $>$ maxval

The maximal values in each bin.

- array_ $\mathrm{t}<$ double $>$ minval 
The minimal values in each bin.

- uint32_t nobs

Number of bitvectors.

\section{Friends}

- class ibis::ambit

- class ibis::band

- class ibis::mesa

- class ibis::mesh

- class ibis::pack

- class ibis::pale

- class ibis::range

- class ibis::zone

\section{Classes}

- struct granule

A data structure to assist the mapping of values to lower precisions.

\subsubsection{Detailed Description}

The equality encoded bitmap index with binning.

The exact bin boundary assignment is controlled by indexing options ' $<$ binning ... $/>$ '.

The 0th bit vector represents $\mathrm{x}<$ bounds[0]; The (nobs-1)st bit vector represents $\mathrm{x}>=$ bounds[nobs-2]; The ith bit vector represents bounds[i-1] $<=\mathrm{x}<$ bounds[i], $(0<\mathrm{i}<$ nbos- 1$)$.

\subsubsection{Member Function Documentation}

\subsubsection{1 void ibis::bin::binning (const char $* \boldsymbol{f}$, const std::vector $<$ double $>\boldsymbol{\&} \boldsymbol{b} \boldsymbol{d}$ ) [ protected]}

Generate bins according to the specified boundaries.

Note:

This function does not attempt to clear the content of the current data structure, the caller is responsible for this task!

3.7.2.2 long ibis::bin::checkBin (const ibis::qRange \& cmp, uint32_t jbin, const ibis::bitvector \& mask, ibis::bitvector \& res) const

Candidate check using the binned values.

The bitvector mask marks the actual values in the bin (because the bitmaps stored in bits do not directly corresponds to the bin).

\subsubsection{3 long ibis::bin::checkBin (const ibis::qRange \& cmp, uint32_t jbin, ibis::bitvector \& res) const}

Candidate check using the binned values.

Returns the number of hits if successful, otherwise it returns a negative value. 
3.7.2.4 void ibis::bin::estimate (const ibis::rangeJoin \& expr, ibis::bitvector64 \& lower, ibis::bitvector64 \& upper) const [virtual]

Estimate the hits for symmetric joins.

Record the definite hits in lower, and all possible hits in upper. NOTE: upper includes all entries in lower.

3.7.2.5 void ibis::bin::estimate (const ibis::qContinuousRange \& expr, ibis::bitvector \& lower, ibis::bitvector \& upper) const [virtual]

Computes an approximation of hits as a pair of lower and upper bounds.

\section{Parameters:}

expr the query expression to be evaluated.

lower a bitvector marking a subset of the hits. All rows marked with one (1) are definitely hits.

upper a bitvector marking a superset of the hits. All hits are marked with one, but some of the rows marked one may not be hits. If the variable upper is empty, the variable lower is assumed to contain the exact answer.

Implements ibis::index.

Reimplemented in ibis::range, ibis::mesa, ibis::ambit, ibis::pale, ibis::pack, ibis::zone, ibis::egale, ibis::moins, and ibis::entre.

3.7.2.6 long ibis::bin::evaluate (const ibis::qContinuousRange \& expr, ibis::bitvector \& hits) const [virtual]

To evaluate the exact hits.

On success, return the number of hits, otherwise a negative value is returned.

Implements ibis::index.

Reimplemented in ibis::range, ibis::mesa, ibis::ambit, ibis::pale, ibis::pack, ibis::zone, ibis::egale, ibis::moins, and ibis::entre.

\subsubsection{7 int ibis::bin::expandRange (ibis::qContinuousRange $\boldsymbol{\&} \boldsymbol{r n g}$ ) const [virtua l]}

The functions expandRange and contractRange expands or contracts the boundaries of a range condition so that the new range will have exact answers using the function estimate.

The default implementation provided does nothing since this is only meaningful for indices based on bins.

Reimplemented from ibis::index.

Reimplemented in ibis::range, ibis::bak, and ibis::bak2.

\subsubsection{8 double ibis::bin::getSum () const [virtual]}

Compute the approximate sum of all the values indexed.

If it decides that computing the sum directly from the vertical partition is more efficient, it will return NaN immediately. Implements ibis::index.

Reimplemented in ibis::range, ibis::mesa, ibis::ambit, ibis::pack, ibis::egale, ibis::moins, and ibis::entre.

\subsubsection{9 void ibis::bin::print (std::ostream \& out) const [virtua l]}

Prints human readable information.

Outputs information about the index as text to the specified output stream. 
Implements ibis::index.

Reimplemented in ibis::range, ibis::mesa, ibis::ambit, ibis::pale, ibis::pack, ibis::zone, ibis::egale, ibis::moins, ibis::entre, ibis::bak, and ibis::bak2.

\subsubsection{0 void ibis::bin::read (int $f d e s$, uint32_t start, const char $* f n$ )}

Read an ibis::bin embedded inside a file.

This is intended to be used by multi-level indices.

Reimplemented in ibis::range.

\subsubsection{1 void ibis::bin::read (ibis::fileManager::storage $*$ st) [virtual]}

Reconstructs an index from an array of bytes.

Intended for internal use only!

Implements ibis::index.

Reimplemented in ibis::range, ibis::ambit, ibis::pale, ibis::pack, ibis::zone, and ibis::egale.

\subsubsection{2 void ibis::bin::read (const char $*$ idxfile) [ virtual]}

Reconstructs an index from the named file.

The name can be the directory containing an index file. In this case, the name of the index file must be the name of the column followed by ".idx" suffix.

Implements ibis::index.

Reimplemented in ibis::range, ibis::ambit, ibis::pale, ibis::pack, ibis::zone, ibis::egale, ibis::bak, and ibis::bak2.

\subsubsection{3 void ibis::bin::setBoundaries (const char $* \boldsymbol{f}$ ) [protected]}

Set bin boundaries.

The bin specification can be of the following, where all fields are optional.

- equal ([_-] ?) [weight|length|ratio])

- no=xxx|nbins=xxx|bins:( ([begin, end, no=xxx $))+$

- <binning (start=begin end=end nbins=xxx scale=[linear|log]) * />

- <binning binFile=file-name[, nbins=xxx] />

The bin speficication can be read from the column object, the table object containing the column, or the global ibis::gParameters object under the name of table-name. column-name. index. If no index specification is found, it builts approximate equal weight bins.

Note:

If equal weight is specified, it take precedence over all other specification.

3.7.2.14 float ibis::bin::undecidable (const ibis::qContinuousRange \& expr, ibis::bitvector \& iffy) const [virtual]

Mark the position of the rows that can not be decided with this index.

\section{Parameters:}

expr the range conditions to be evaluated. 
iffy the bitvector marking the positions of rows that can not be decided using the index. Return value is the expected fraction of undecided rows that might satisfy the range conditions.

Implements ibis::index.

Reimplemented in ibis::range, ibis::mesa, ibis::ambit, ibis::pale, ibis::pack, ibis::zone, and ibis::egale.

\subsubsection{5 void ibis::bin::write (const char $* \boldsymbol{d t}$ ) const [virtual]}

Save index to a file.

Outputs the index in a compact binary format to the named file or directory. The index file contains a header that can be identified by the function isIndex.

Implements ibis::index.

Reimplemented in ibis::range, ibis::mesa, ibis::ambit, ibis::pale, ibis::pack, ibis::zone, ibis::egale, ibis::moins, ibis::entre, ibis::bak, and ibis::bak2.

The documentation for this class was generated from the following files:

- ibin.h

- ibin.cpp

\section{8 ibis::bin::granule Struct Reference}

A data structure to assist the mapping of values to lower precisions.

\#include <ibin.h>

\section{Public Attributes}

- ibis::bitvector $*$ loc0

- ibis: :bitvector $*$ loc1

- double max0

- double max1

- double min0

- double $\min 1$

\subsubsection{Detailed Description}

A data structure to assist the mapping of values to lower precisions.

Any integral or floating-point value may be mapped to lower precision floating-point value. This would produce a more granular representation of the values. The low precision floating-point value is called a target in this description. To facilitate this type of dynamic binning, we device this simple data structure to record the position of all records mapped to a particular target value. It separates out the values that are larger than or equal to the target from those that are smaller than the target. The variables $\min 0$ and $\max 0$ store the actual minimum and maximum value among those that are smaller than the target. The variables min1 and max 1 store the actual minimum and maximum value among those that are larger than or equal to the target value.

The documentation for this struct was generated from the following file:

- ibin.h 


\section{9 ibis::bitvector Class Reference}

A data structure to represent a sequence of bits.

\#include <bitvector.h>

\section{Public Types}

- typedef uint32_t word_t

The basic unit of data storage.

\section{Public Member Functions}

- void adjustSize (word_t nv, word_t nt)

Adjust the size of the bit sequence.

- void appendFill (int val, word_t $\mathrm{n}$ )

Append $\mathrm{n}$ bits of val.

- void appendWord (word_t w)

Append a word of bits.

- const_iterator begin () const

- iterator begin ()

- bitvector (const char $*$ file)

Read the content of the named file.

- bitvector (const array_t $<$ word_t $>$ \&arr)

Construct a bitvector from an array.

- bitvector (const bitvector \&bv)

Shallow copy. Underlying storage is reference counted.

- uint32_t bytes () const throw ()

Return the number of bytes used by the bitvector object in memory.

- void clear ()

Remove the existing content of a bitvector.

- word_t cnt () const

Return the number of bits that are one.

- void compress ()

Merge fills into fill words.

- word_t compressible () const

Return the number of word saved if the function compress is called.

- bitvector \& copy (const bitvector \&bv)

Note:

Deep copy. 
- void decompress ()

Turn all fill words into literal words.

- const_iterator end () const

- iterator end ()

- void erase (word_t $\mathrm{i}$, word_t $\mathrm{j}$ )

Remove the bits in the range of $[i, j)$.

- indexSet firstIndexSet () const

- void flip ()

Bitwise operations.

- uint32_t getSerialSize () const throw ()

Compute the number of word in serialized version of the bitvector object.

- word_t numFillWords () const

Return the number of fill words.

- bitvector $*$ operator $\&$ (const bitvector \&) const

- void operator $\&=$ (const bitvector \&rhs)

Perform bitwise AND between this bitvector and rhs.

- bitvector \& operator+= (int b)

Append a single bit.

- bitvector \& operator+= (const bitvector \&bv)

Append a bitvector.

- bitvector $*$ operator- (const bitvector \&) const

- void operator-= (const bitvector \&rhs)

Perform bitwise subtraction $(a \& ! b)$.

- const bitvector \& operator= (const bitvector \&bv)

Note:

Deep copy.

- int operator== (const bitvector \&rhs) const

- bitvector $*$ operator $^{\wedge}$ (const bitvector $\&$ ) const

- void operator ${ }^{\wedge}=($ const bitvector \&rhs)

Perform bitwise exclusive or (XOR).

- bitvector $*$ operator| (const bitvector \&) const

- void operator $\mid=$ (const bitvector \&rhs)

Perform bitwise OR.

- std::ostream \& print (std::ostream \&) const

The print function.

- void read (const char $* \mathrm{fn}$ )

I/O functions.

- void reserve (unsigned $\mathrm{nb}$, unsigned $\mathrm{nc}$, double $\mathrm{cf}=0.0$ )

- void set (int val, word_t $n$ ) 
Create a vector with $\mathrm{n}$ bits of value val (cf.

- void setBit (const word_t i, int val)

Replace a single bit at position i.

- void setSize (word_t $\mathrm{n}$ ) const

Explicitly set the size of the bitvector.

- word_t size () const throw ()

Return the total number of bits in the bit sequence.

- bitvector \& swap (bitvector \&bv)

- void turnOnRawBit (const word_t i)

Turn on a single bit in a uncompressed bitvector.

- void write (array_t $\mathrm{t}$ word_t $>$ \&arr) const

Write the bit vector to an array_t $t<w o r d \_t>$.

- void write (int fdes) const

Write to a file that is opened by the caller.

- void write (const char $* \mathrm{fn})$ const

Write the bit vector to a file.

\section{Static Public Member Functions}

- static word_t bitsPerLiteral ()

Return the number of bits in a literal word.

- static double clusteringFactor (word_t nb, word_t nc, word_t sz)

Estimate clustering factor based on the size.

- static double markovSize (word_t nb, word_t nc, double f)

Compute the expected size (number of bytes) of a random sequence generated from a Markov process.

- static double randomSize (word_t nb, word_t nc)

Compute the expected number of bytes required to store a random sequence.

\section{Protected Member Functions}

- bool all0s () const

Are all bits in regular words 0 ?

- bool all1s () const

Are all bits in regular words 1 ?

- bool isCompressed () const 


\section{Friends}

- struct active_word

- class const_iterator

- class indexSet

- class iterator

- struct run

\section{Classes}

- struct active_word

The struct active_word stores the last few bits that do not fill a whole word.

- class const_iterator

The const_iterator class. It iterates on the individual bits.

- class indexSet

The indexSet stores positions of bits that are one.

- class iterator

The iterator that allows modification of bits.

- struct run

An internal struct used during logical operations to track the usage of fill words.

\subsubsection{Detailed Description}

A data structure to represent a sequence of bits.

Key features

A bitvector object stores a sequence of bits and provides fast bitwise logical operations. In addition, it supports operations to append new bits from the end, read bits at arbitrary location and set bits at arbitrary location. It also supports an iterator, a const_iterator and an indexSet.

Encoding format

Incoming bits are organized into words (bitvector::word_t). A word is a literal word if its Most Significant Bit (MSB) is 0 , it is a fill word if its MSB is 1 . A literal word stores literal bit values in the bit position following the MSB and a fill word stores a sequence of consecutive bits that are of the same value, i.e., a fill. The second most significant bit of the fill word is the bit value, the remaining bits of the word is a unsigned integer that stores the length of the fill as number of equivalent literal words, i.e., how many literal words it will take if the fill is stored in literal words.

Restrictions

- The number of bits must be expressible by one single bitvector::word_t. This ensure that a fill word can store a fill of any valid length without performing a bound check. If bitvector::word_t is 32-bit long, the maximum number of bits that can be represented by a bitvector object is 4 billion.

- When adding a bit with bitvector::operator+=, the integer value passed in must be one of 0 or 1 . Since checking whether the import value is 0 or not 0 causes pipeline bubble in CPU, we have opted for not performing the check. An input value other than 0 or 1 will cause existing bits to be modified in unpredictable ways. 


\subsubsection{Constructor \& Destructor Documentation}

\subsubsection{1 ibis::bitvector::bitvector (const array_t $<$ word_t $>\boldsymbol{\&}$ arr) $\quad$ [inline ]}

Construct a bitvector from an array.

Because the array copy constructor performs shallow copy, this bitvector is not using any new space.

\subsubsection{Member Function Documentation}

\subsubsection{1 void ibis::bitvector::adjustSize (word_t $n v$, word_t $n t$ )}

Adjust the size of the bit sequence.

If current size is less than nv, append enough 1 bits so that it has nv bits. If the resulting total number of bits is less than $n t$, append 0 bits so that there are $n t$ total bits. The final result always contains $n t$ bits.

\subsubsection{2 double ibis::bitvector::clusteringFactor (word_t $\boldsymbol{n} \boldsymbol{b}$, word_t $\boldsymbol{n} \boldsymbol{c}$, word_t $\boldsymbol{s z}$ ) [ stat ic ]}

Estimate clustering factor based on the size.

The size is measured as the number of bytes. markovSize.

\subsubsection{3 void ibis::bitvector::flip ()}

Bitwise operations.

Complement all bits of a bit sequence.

\subsubsection{4 uint32_t ibis::bitvector::getSerialSize () const throw () [inline ]}

Compute the number of word in serialized version of the bitvector object.

This would be the size of this bitvector object on disk or in a single array_t $<$ word_t $>$.

\subsubsection{5 double ibis::bitvector::markovSize (word_t $\boldsymbol{n} \boldsymbol{b}$, word_t $\boldsymbol{n} \boldsymbol{c}$, double $\boldsymbol{f}$ ) [inline, static ]}

Compute the expected size (number of bytes) of a random sequence generated from a Markov process.

The bit sequence is to have $n b$ total bits, $n c$ bits of one, and $f$ consecutive ones on the average. The argument $f$ is known as the clustering factor.

\subsubsection{6 const ibis::bitvector $\boldsymbol{\&}$ ibis::bitvector::operator= (const bitvector $\boldsymbol{\&} \boldsymbol{b} v) \quad$ [in 1 ine ]}

Note:

Deep copy.

Use deep copy. Wanted to use shallow copy for efficiency considerations, but SHALLOW copy causes unexpected problem in test program bitty.cpp.

\subsubsection{7 double ibis::bitvector::randomSize (word_t $\boldsymbol{n} \boldsymbol{b}$, word_t $\boldsymbol{n c}$ ) [inline, static ]}

Compute the expected number of bytes required to store a random sequence.

The random bit sequence is to have nb total bits and nc bits of one. 


\subsubsection{8 void ibis::bitvector::read (const char $* f n$ )}

I/O functions.

Read a bit vector from the file. Purge current contents before read.

\subsubsection{9 void ibis::bitvector::set (int val, word_t $n$ )}

Create a vector with $\mathrm{n}$ bits of value val (cf.

memset()).

Note:

val must be either 0 or 1 .

\subsubsection{0 void ibis::bitvector::setBit (const word_t $i$, int $v a l$ )}

Replace a single bit at position $i$.

Note:

val must be either 0 or 1 .

\subsubsection{1 void ibis::bitvector::setSize (word_t $\boldsymbol{n}$ ) const [i n l i ne ]}

Explicitly set the size of the bitvector.

Caller is responsible for ensuring the size assigned is actually correct.

\subsubsection{2 void ibis::bitvector::write (array_t $<$ word_t $>$ \& arr) const}

Write the bit vector to an array_t $<$ word_t $\mathrm{t}$.

The serialize version of the bit vector may be passed to another I/O function or sent through networks.

The documentation for this class was generated from the following files:

- bitvector.h

- bitvector.cpp

\subsection{0 ibis::bitvector64 Class Reference}

A data structure to represent a sequence of bits.

\#include <bitvector $64 . \mathrm{h}>$

\section{Public Types}

- typedef uint64_tword_t

The basic unit of data storage is 64-bit.

\section{Public Member Functions}

- void adjustSize (word_t nv, word_t nt)

Adjust the size of the bit sequence. 
- void appendFill (int val, word_t n)

Append $\mathrm{n}$ bits of $\mathrm{val}$.

- void appendWord (word_t w)

Append a word of bits.

- const_iterator begin () const

- iterator begin ()

- bitvector64 (const char $*$ file)

Read the content of the named file.

- bitvector64 (const array_t $<$ word_t $>$ \&arr)

- bitvector64 (const bitvector64 \&bv)

- word_t bytes () const throw ()

Return the number of bytes used by the bitvector object in memory.

- void clear ()

Remove the existing content of a bitvector64.

- word_t cnt () const

Return the number of bits that are one.

- $\operatorname{void}$ compress ()

Merge fills into fill words.

- word_t compressible () const

Return the number of word saved if the function compress is called.

- bitvector64 \& copy (const bitvector64 \&bv)

Note:

Deep copy.

- void decompress ()

Turn all fill words into literal words.

- const_iterator end () const

- iterator end ()

- void erase (word_t $\mathrm{i}$, word_t $\mathrm{j}$ )

Remove the bits in the range of $[i, j)$.

- indexSet firstIndexSet () const

- $\operatorname{void}$ flip ()

Bitwise operations.

- word_t getSerialSize () const throw ()

Compute the number of words in serialized version of the bitvector object.

- word_t numFillWords () const

Return the number of fill words.

- bitvector64 * operator \& (const bitvector64 \&) const

- void operator $\&=$ (const bitvector64 \&rhs) 
Perform bitwise AND between this bitvector64 and rhs.

- bitvector64 \& operator+= (int b)

Append a single bit.

- bitvector64 \& operator+= (const bitvector64 \&bv)

Append a bitvector64.

- bitvector64 $*$ operator- (const bitvector64 \&) const

- void operator-= (const bitvector64 \&rhs)

Perform bitwise subtraction ( $a \& ! b$ ).

- bitvector64 \& operator= (const bitvector64 \&bv)

Note:

Deep copy.

- int operator $==$ (const bitvector64 \&rhs) const

- bitvector64 $*$ operator $^{\wedge}$ (const bitvector64 \&) const

- void operator $^{\wedge}=($ const bitvector64 \&rhs)

Perform bitwise exclusive or (XOR).

- bitvector64 $*$ operator| (const bitvector64 \&) const

- void operator $\mid=$ (const bitvector64 \&rhs)

Perform bitwise OR.

- std::ostream \& print (std::ostream \&) const

The print function.

- void read (const char $* \mathrm{fn}$ )

I/O functions.

- void set (int val, word_t $\mathrm{n}$ )

Create a vector with $\mathrm{n}$ bits of value val (cf.

- void setBit (const word_t i, int val)

Replace a single bit at position i.

- word_t size () const throw ()

Return the total number of bits in the bit sequence.

- bitvector64 \& swap (bitvector64 \&bv)

- void write (array_t $<$ word_t $>\&$ arr) const

Write the bit vector to an array_t $t<w o r d \_t>$.

- void write (FILE $*$ fptr) const

- void write (const char $* \mathrm{fn}$ ) const

Write the bit vector to a file. 


\section{Static Public Member Functions}

- static unsigned bitsPerLiteral ()

Return the number of bits in a literal word.

- static double clusteringFactor (word_t nb, word_t nc, word_t nw)

Estimate clustering factor based on the size.

- static double markovSize (word_t nb, word_t nc, double f)

Compute the expected size (bytes) of a random sequence generated from a Markov process.

- static double randomSize (word_t nb, word_t nc)

Compute the expected number of bytes required to store a random sequence.

\section{Protected Member Functions}

- bool all0s () const

Are all bits in regular words 0 ?

- bool all1s () const

Are all bits in regular words 1 ?

- bool isCompressed () const

\section{Friends}

- struct active_word

- class const_iterator

- class indexSet

- class iterator

- struct run

\section{Classes}

- struct active_word

The struct active_word stores the last few bits that do not fill a whole word.

- class const_iterator

The const_iterator class. It iterates on the individual bits.

- class indexSet

The indexSet stores positions of bits that are one.

- class iterator

The iterator that allows modification of bits.

- struct run

An internal struct used during logical operations to track the usage of fill words. 


\subsubsection{Detailed Description}

A data structure to represent a sequence of bits.

The 64-bit version.

Key features

A bitvector object stores a sequence of bits and provides fast bitwise logical operations. In addition, it supports operations to append new bits from the end, read bits at arbitrary location and set bits at arbitrary location. It also supports an iterator, a const_iterator and an indexSet.

Encoding format

Incoming bits are organized into words (bitvector::word_t). A word is a literal word if its Most Significant Bit (MSB) is 0 , it is a fill word if its MSB is 1 . A literal word stores literal bit values in the bit position following the MSB and a fill word stores a sequence of consecutive bits that are of the same value, i.e., a fill. The second most significant bit of the fill word is the bit value, the remaining bits of the word is a unsigned integer that stores the length of the fill as number of equivalent literal words, i.e., how many literal words it will take if the fill is stored in literal words.

Restrictions

- The number of bits must be expressible by one single bitvector::word_t. This ensure that a fill word can store a fill of any valid length without performing a bound check. In this 64-bit version, the maximum number of bits that can be represented by a bitvector object is 16 quintillion $\left(16 \times 10^{\wedge}\{18\}\right)$.

- When adding a bit with bitvector::operator+=, the integer value passed in must be one of 0 or 1 . Since checking whether the import value is 0 or not 0 causes pipeline bubble in CPU, we have opted for not performing the check. An input value other than 0 or 1 will cause existing bits to be modified in unpredictable ways.

\subsubsection{Member Function Documentation}

\subsubsection{1 void ibis::bitvector64::adjustSize (word_t $n v$, word_t $n t$ )}

Adjust the size of the bit sequence.

If current size is less than nv, append enough 1 bits so that it has nv bits. If the resulting total number of bits is less than $n t$, append 0 bits so that there are $n t$ total bits. The final result always contains $n t$ bits.

\subsubsection{2 double ibis::bitvector64::clusteringFactor (word_t $\boldsymbol{n} \boldsymbol{b}$, word_t $\boldsymbol{n} \boldsymbol{c}$, word_t $\boldsymbol{n} \boldsymbol{w}$ ) [ stat ic ]}

Estimate clustering factor based on the size.

markovSize.

\subsubsection{3 void ibis::bitvector64::flip ()}

Bitwise operations.

Complement all bits of a bit sequence.

\subsubsection{4 double ibis::bitvector64::markovSize (word_t $\boldsymbol{n} \boldsymbol{b}$, word_t $\boldsymbol{n} \boldsymbol{c}$, double $\boldsymbol{f}$ ) [ in line, static ]}

Compute the expected size (bytes) of a random sequence generated from a Markov process.

The bit sequence is to have $\mathrm{nb}$ total bits, $\mathrm{nc}$ bits of one, and $\mathrm{f}$ consecutive ones on the average. The argument $f$ is known as the clustering factor.

\subsubsection{5 double ibis::bitvector64::randomSize (word_t $\boldsymbol{n} \boldsymbol{b}$, word_t $\boldsymbol{n c}$ ) [inline, stat ic]}

Compute the expected number of bytes required to store a random sequence.

The random bit sequence is to have nb total bits and nc bits of one. 


\subsubsection{6 void ibis::bitvector64::read (const char $* f n$ )}

I/O functions.

Read a bit vector from the file. Purge current contents before read.

\subsubsection{7 void ibis::bitvector64::set (int val, word_t $n$ )}

Create a vector with $\mathrm{n}$ bits of value val (cf.

memset()).

Note:

val must be either 0 or 1 .

\subsubsection{8 void ibis::bitvector64::setBit (const word_t $i$, int val)}

Replace a single bit at position $i$.

Note:

val must be either 0 or 1 .

The documentation for this class was generated from the following files:

- bitvector64.h

- bitvector64.cpp

\subsection{1 ibis::bitvector64::const_iterator Class Reference}

The const_iterator class. It iterates on the individual bits.

\#include <bitvector64.h>

\section{Public Member Functions}

- bool operator $*()$ const

- int operator!= (const const_iterator \&rhs) const throw ()

- const_iterator \& operator++ ()

- const_iterator \& operator+= (int64_t incr)

- const_iterator \& operator- ()

- int operator== (const const_iterator \&rhs) const throw ()

\section{Friends}

- const_iterator ibis::bitvector64::begin () const

- const_iterator ibis::bitvector64::end () const

- class ibis::bitvector64::iterator

\subsubsection{Detailed Description}

The const_iterator class. It iterates on the individual bits.

The documentation for this class was generated from the following files:

- bitvector64.h

- bitvector64.cpp 


\subsection{2 ibis::bitvector64::indexSet Class Reference}

The indexSet stores positions of bits that are one.

\#include <bitvector $64 . \mathrm{h}>$

\section{Public Member Functions}

- const word_t $*$ indices () const

- bool isRange () const

- word_t nIndices () const

- indexSet \& operator++ ()

\section{Friends}

- indexSet ibis::bitvector64::firstIndexSet () const

\subsubsection{Detailed Description}

The indexSet stores positions of bits that are one.

It decodes one word of the bitvector64 as a time. For a fill of ones, the function isRange returns true, otherwise it returns false. If isRange returns true, the position of the first bit is pointed by the pointer returned by function indices, and there are nIndices consecutive ones. If isRange returns false, there are nIndices bits that are one and the positions of these bits are stored in the array returned by function indices.

The documentation for this class was generated from the following files:

- bitvector64.h

- bitvector64.cpp

\subsection{3 ibis::bitvector64::iterator Class Reference}

The iterator that allows modification of bits.

\#include <bitvector64.h>

\section{Public Member Functions}

- bool operator $*()$ const

- int operator!= (const iterator \&rhs) const throw ()

- int operator!= (const const_iterator \&rhs) const throw ()

- iterator \& operator++ ()

- iterator \& operator+= (int64_t incr)

- iterator \& operator-()

- iterator \& operator= (int val)

- int operator $==$ (const iterator \&rhs) const throw ()

- int operator== (const const_iterator \&rhs) const throw ()

\section{Friends}

- iterator ibis::bitvector64::begin ()

- iterator ibis::bitvector64::end ()

- void ibis::bitvector64::erase (word_t i, word_t j) 


\subsubsection{Detailed Description}

The iterator that allows modification of bits.

It provides only one additional function (operator=) than const_iterator to allow modification of the bit pointed.

IMPORTANT: operator= modifies the content of the bitvector64 it points to and it can invalidate other iterator or const_iterator referring to the same bitvector64.

The documentation for this class was generated from the following files:

- bitvector64.h

- bitvector64.cpp

\subsection{4 ibis::bitvector::const_iterator Class Reference}

The const_iterator class. It iterates on the individual bits.

\#include <bitvector.h>

\section{Public Member Functions}

- const_iterator (const const_iterator \&r)

- bool operator $*()$ const

- int operator!= (const const_iterator \&rhs) const throw ()

- const_iterator \& operator++ ()

- const_iterator \& operator+= (int incr)

- const_iterator \& operator- ()

- const_iterator \& operator= (const const_iterator \&r)

- int operator== (const const_iterator \&rhs) const throw ()

\section{Friends}

- const_iterator ibis::bitvector::begin () const

- const_iterator ibis::bitvector::end () const

- class ibis::bitvector::iterator

\subsubsection{Detailed Description}

The const_iterator class. It iterates on the individual bits.

The documentation for this class was generated from the following files:

- bitvector.h

- bitvector.cpp

\subsection{5 ibis::bitvector::indexSet Class Reference}

The indexSet stores positions of bits that are one.

\#include <bitvector.h> 


\section{Public Member Functions}

- const word_t $*$ indices () const

- bool isRange () const

- word_t nIndices () const

- indexSet \& operator++ ()

\section{Friends}

- indexSet ibis::bitvector::firstIndexSet () const

\subsubsection{Detailed Description}

The indexSet stores positions of bits that are one.

It decodes one word of the bitvector at a time. For a fill of ones, the function isRange returns true, otherwise it returns false. If isRange returns true, the position of the first bit is pointed by the pointer returned by function indices, and there are nIndices consecutive ones. If isRange returns false, there are nIndices bits that are one and the positions of these bits are stored in the array returned by function indices.

The documentation for this class was generated from the following files:

- bitvector.h

- bitvector.cpp

\subsection{6 ibis::bitvector::iterator Class Reference}

The iterator that allows modification of bits.

\#include <bitvector.h>

\section{Public Member Functions}

- iterator (const iterator \&r)

- bool operator $*()$ const

- int operator!= (const iterator \&rhs) const throw ()

- int operator!= (const const_iterator \&rhs) const throw ()

- iterator \& operator++ ()

- iterator \& operator+= (int incr)

- iterator \& operator- ()

- const iterator \& operator= (int val)

- const iterator \& operator= (const iterator \&r)

- int operator $==$ (const iterator \&rhs) const throw ()

- int operator== (const const_iterator \&rhs) const throw ()

\section{Friends}

- iterator ibis::bitvector::begin ()

- iterator ibis::bitvector::end ()

- void ibis::bitvector::erase (word_t i,word_t j) 


\subsubsection{Detailed Description}

The iterator that allows modification of bits.

It provides only one additional function (operator=) than const_iterator to allow modification of the bit pointed.

IMPORTANT: operator= modifies the content of the bitvector it points to and it can invalidate other iterators or const_iterators referring to the same bitvector.

The documentation for this class was generated from the following files:

- bitvector.h

- bitvector.cpp

\subsection{7 ibis::bord Class Reference}

Class ibis::bord stores all its data in memory.

\#include $<$ bord.h $>$

Inheritance diagram for ibis::bord::

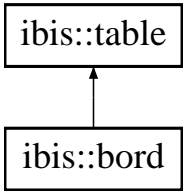

\section{Public Types}

- typedef std::vector $<$ void $*>$ bufferList

\section{Public Member Functions}

- bord (const char $*$ tn, const char $*$ td, uint64_t nr, const ibis::table::stringList \&cn, const ibis::table::typeList \&ct, const bufferList \&buf)

- virtual int buildIndex (const char $*$, const char $*$ )

Create the index for named column.

- virtual int buildIndexes (const char *)

Create indexes for every column of the table.

- virtual ibis::table::stringList columnNames () const

Return column names.

- virtual ibis::table::typeList columnTypes () const

Return data types.

- virtual ibis::table::cursor $*$ createCursor () const

Create a cursor object to perform row-wise data access.

- virtual void describe (std::ostream \&) const

Print a description of the table to the specified output stream.

- virtual int dump (std::ostream \&, const char $*$ ) const 
Dump the values in ASCII form to the specified output stream.

- virtual void estimate (const char *cond, uint64_t \&nmin, uint64_t \&nmax) const

Estimate the number of rows satisfying the selection conditions.

- virtual int64_t getColumnAsBytes (const char $*$, char $*$ ) const

Retrieve all values of the named column.

- virtual int64_t getColumnAsDoubles (const char $*$, double $*$ ) const

Retrieve all values of the named column.

- virtual int64_t getColumnAsFloats (const char $*$, float $*$ ) const

Retrieve all values of the named column.

- virtual int64_t getColumnAsInts (const char $*$, int32_t $*$ ) const

Retrieve all values of the named column.

- virtual int64_t getColumnAsLongs (const char $*$, int64_t $*$ ) const

Retrieve all values of the named column.

- virtual int64_t getColumnAsShorts (const char $*$, int16_t *) const

Retrieve all values of the named column.

- virtual int64_t getColumnAsStrings (const char $*$, std::vector $<$ std::string $>\&$ ) const

Retrieve the null-terminated strings as a vector of std::string objects.

- virtual int64_t getColumnAsUBytes (const char $*$, unsigned char $*$ ) const

Retrieve all values of the named column.

- virtual int64_t getColumnAsUInts (const char $*$, uint32_t *) const

Retrieve all values of the named column.

- virtual int64_t getColumnAsULongs (const char $*$, uint64_t $*$ ) const

Retrieve all values of the named column.

- virtual int64_t getColumnAsUShorts (const char $*$, uint16_t $*$ ) const

Retrieve all values of the named column.

- virtual long getHistogram (const char $*$, const char $*$, double, double, double, std::vector $<$ size_t $>\&$ ) const

Compute the histogram of the named column.

- virtual long getHistogram2D (const char $*$, const char $*$, double, double, double, const char $*$, double, double, double, std::vector $<$ size_t $>\&$ ) const

Compute a two-dimension histogram on columns cname1 and name2.

- virtual long getHistogram3D (const char $*$, const char $*$, double, double, double, const char $*$, double, double, double, const char $*$, double, double, double, std::vector $<$ size_t $>\&$ ) const

Compute a three-dimensional histogram on the named columns.

- virtual table $*$ groupby (const ibis::table::stringList \&) const

Perform aggregate functions on the current table.

- virtual void indexSpec (const char $*$, const char $*$ ) 
Replace the current indexing option.

- virtual const char $*$ indexSpec (const char $*$ ) const

Retrieve the current indexing option.

- virtual size_t nColumns () const

- virtual uint64_t nRows () const

- virtual void orderby (const ibis::table::stringList \&)

Reorder the rows.

- virtual void reverseRows ()

Reverse the order of the rows.

- virtual table $*$ select (const char $*$ sel, const char $*$ cond) const

Given a set of column names and a set of selection conditions, compute another table that represents the selected values.

\section{Protected Member Functions}

- void clear ()

Clear the existing content.

- int64_t computeHits (const char *cond) const

Compute the number of hits.

\section{Protected Attributes}

- part mypart

\section{Friends}

- class cursor

\section{Classes}

- class column

An in-memory version of ibis::column.

- class cursor

- class part

\subsubsection{Detailed Description}

Class ibis::bord stores all its data in memory.

The function ibis: : table: : select produces an ibis: :bord object if the query produce nontrivial results.

Note:

Bord is the Danish word for "table." 


\subsubsection{Member Function Documentation}

\subsubsection{1 virtual int ibis::bord::buildIndex (const char $*$, const char $*$ ) [inline, virtual]}

Create the index for named column.

The existing index will be replaced. If an indexing option is not specified, it will use the internally recorded option for the named column or the table containing the column.

Note:

Unless any there is a specific instruction to not index a column, the querying functions will automatically build indices as necessary. However, as building an index is relatively expensive process, building an index on a column is on average about four or five times as expensive as reading the column from disk, this function is provided so that it is possible to build indexes beforehand.

Implements ibis::table.

\subsubsection{2 virtual int ibis::bord::buildIndexes (const char $*$ ) [inline, virtual]}

Create indexes for every column of the table.

Existing indexes will be replaced. If an indexing option is not specified, the internally recorded options will be used. buildIndex

Implements ibis::table.

\subsubsection{3 int ibis::bord::dump (std::ostream \&, const char $*$ ) const [inline, virtual]}

Dump the values in ASCII form to the specified output stream.

The default delimiter is coma (","), which produces Comma-Separated-Values (CSV).

Implements ibis::table.

\subsubsection{4 void ibis::bord::estimate (const char $*$ cond, uint64_t \& nmin, uint64_t \& nmax) const [virtua l]}

Estimate the number of rows satisfying the selection conditions.

The number of rows is between [nmin, nmax].

Implements ibis::table.

\subsubsection{5 int64_t ibis::bord::getColumnAsBytes (const char $*$, char $*$ ) const [virtual]}

Retrieve all values of the named column.

The member functions of this class only support access to whole column at a time. Use table: : cursor class for row-wise accesses. For fixed-width data types, the raw pointers are used to point to the values to be returned. In these cases, the caller is responsible for allocating enough storage for the values to be returned.

Implements ibis::table.

\subsubsection{6 int64_t ibis::bord::getColumnAsDoubles (const char $*$, double $*$ ) const [ virtua l]}

Retrieve all values of the named column.

The member functions of this class only support access to whole column at a time. Use table: : cursor class for row-wise accesses. For fixed-width data types, the raw pointers are used to point to the values to be returned. In these cases, the caller is responsible for allocating enough storage for the values to be returned.

Implements ibis::table. 


\subsubsection{7 int64_t ibis::bord::getColumnAsFloats (const char $*$, float $*$ ) const [virtual]}

Retrieve all values of the named column.

The member functions of this class only support access to whole column at a time. Use table: : cursor class for row-wise accesses. For fixed-width data types, the raw pointers are used to point to the values to be returned. In these cases, the caller is responsible for allocating enough storage for the values to be returned.

Implements ibis::table.

\subsubsection{8 int64_t ibis::bord::getColumnAsInts (const char $*$, int32_t $*$ ) const [virtua l]}

Retrieve all values of the named column.

The member functions of this class only support access to whole column at a time. Use table: : cursor class for row-wise accesses. For fixed-width data types, the raw pointers are used to point to the values to be returned. In these cases, the caller is responsible for allocating enough storage for the values to be returned.

Implements ibis::table.

\subsubsection{9 int64_t ibis::bord::getColumnAsLongs (const char $*$, int64_t $*$ ) const [virtual]}

Retrieve all values of the named column.

The member functions of this class only support access to whole column at a time. Use table: : cursor class for row-wise accesses. For fixed-width data types, the raw pointers are used to point to the values to be returned. In these cases, the caller is responsible for allocating enough storage for the values to be returned.

Implements ibis::table.

\subsubsection{0 int64_t ibis::bord::getColumnAsShorts (const char $*$, int16_t $*$ ) const ～[virtual]}

Retrieve all values of the named column.

The member functions of this class only support access to whole column at a time. Use table: : cursor class for row-wise accesses. For fixed-width data types, the raw pointers are used to point to the values to be returned. In these cases, the caller is responsible for allocating enough storage for the values to be returned.

Implements ibis::table.

3.17.2.11 int64_t ibis::bord::getColumnAsStrings (const char $*$, std::vector $<$ std::string $>\&$ ) const [virtual]

Retrieve the null-terminated strings as a vector of std::string objects.

Both ibis::CATEGORY and ibis::TEXT types can be retrieved using this function.

Implements ibis::table.

\subsubsection{2 int64_t ibis::bord::getColumnAsUBytes (const char $*$, unsigned char $*$ ) const [virtua l ]}

Retrieve all values of the named column.

The member functions of this class only support access to whole column at a time. Use table: : cursor class for row-wise accesses. For fixed-width data types, the raw pointers are used to point to the values to be returned. In these cases, the caller is responsible for allocating enough storage for the values to be returned.

Implements ibis::table.

\subsubsection{3 int64_t $\mathbf{t}$ ibis::bord::getColumnAsUInts (const char $*$, uint32_t $*$ ) const $\quad$ [virtual]}

Retrieve all values of the named column. 
The member functions of this class only support access to whole column at a time. Use table: : cursor class for row-wise accesses. For fixed-width data types, the raw pointers are used to point to the values to be returned. In these cases, the caller is responsible for allocating enough storage for the values to be returned.

Implements ibis::table.

\subsubsection{4 int64_t ibis::bord::getColumnAsULongs (const char $*$, uint64_t $*$ ) const [virtual]}

Retrieve all values of the named column.

The member functions of this class only support access to whole column at a time. Use table: : cursor class for row-wise accesses. For fixed-width data types, the raw pointers are used to point to the values to be returned. In these cases, the caller is responsible for allocating enough storage for the values to be returned.

Implements ibis::table.

\subsubsection{5 int64_t ibis::bord::getColumnAsUShorts (const char $*$, uint16_t $*$ ) const [virtua ] ]}

Retrieve all values of the named column.

The member functions of this class only support access to whole column at a time. Use table: : cursor class for row-wise accesses. For fixed-width data types, the raw pointers are used to point to the values to be returned. In these cases, the caller is responsible for allocating enough storage for the values to be returned.

Implements ibis::table.

3.17.2.16 long ibis::bord::getHistogram (const char $*$, const char $*$, double, double, double, std::vector $<$ size_t $>$ \&) const [virtual]

Compute the histogram of the named column.

This version uses the user specified bins: [begin, begin+stride) [begin+stride, begin $+2 *$ stride), .... A record is placed in bin ( $x$ - begin) / stride, where the first bin is bin 0. This gives a total of (end - begin) / stride bins.

Note:

Records (rows) outside of the range [begin, end] are not counted.

Non-positive stride is considered as an error.

If end is less than begin, an empty array counts is returned along with return value 0 .

Implements ibis::table.

3.17.2.17 long ibis::bord::getHistogram2D (const char $*$, const char $*$, double, double, double, const char $*$, double, double, double, std::vector $<$ size_t $>$ \&) const $\quad$ [virtual ]

Compute a two-dimension histogram on columns cname1 and name2.

The bins along each dimension are defined the same way as in function getHistogram. The array counts stores the two-dimensional bins with the first dimension as the slow varying dimension following $\mathrm{C}$ convention for ordering multi-dimensional arrays.

Implements ibis::table.

3.17.2.18 long ibis::bord::getHistogram3D (const char $*$, const char $*$, double, double, double, const char $*$, double, double, double, const char $*$, double, double, double, std::vector $<$ size_t $>\&$ ) const [virtua l]

Compute a three-dimensional histogram on the named columns.

The triplets $<$ begin, end, stride $>$ are used the same ways in getHistogram and getHistogram2D. The three dimensional bins are linearized in counts with the first being the slowest varying dimension and the third being the fastest varying dimension following the $\mathrm{C}$ convention for ordering multi-dimensional arrays.

Implements ibis::table. 
3.17.2.19 ibis::table $*$ ibis::bord::groupby (const ibis::table::stringList $\boldsymbol{\&}$ ) const [inline, virtual]

Perform aggregate functions on the current table.

It produces a new table. The list of strings passed to this function are interpreted as a set of names followed by a set of functions. Currently, only functions COUNT, AVG, MIN, MAX, and SUM are supported.

Implements ibis::table.

\subsubsection{0 virtual void ibis::bord::indexSpec (const char $*$, const char $*$ ) [inline, virtual]}

Replace the current indexing option.

If no column name is specified, it resets the indexing option for the table.

Implements ibis::table.

\subsubsection{1 virtual const char $*$ ibis::bord::indexSpec (const char $*$ ) const [inline, virtual]}

Retrieve the current indexing option.

If no column name is specified, it retrieve the indexing option for the table.

Implements ibis::table.

\subsubsection{2 void ibis::bord::orderby (const ibis::table::stringList \&) [inline, virtual]}

Reorder the rows.

Sort the rows in ascending order of the columns specified in the list of column names. This function is not designated const because though it does not change the content in SQL logic, but it may change internal representations.

\section{Note:}

If an empty list is passed to this function, it will reorder rows using all columns with the column having the smallest number of distinct values first.

Implements ibis::table.

The documentation for this class was generated from the following files:

- bord.h

- bord.cpp

\subsection{8 ibis::bord::column Class Reference}

An in-memory version of ibis::column.

\#include <bord.h>

\section{Public Member Functions}

- column (const column \&rhs)

- column (const ibis::bord::part $*$, const ibis::column \&, void $*$ buf)

Note:

Transfer the ownership of st to the new column object.

- column (const ibis::bord::part $*$ tbl, ibis::TYPE_T t, const char $*$ name, void $*$ buf, const char $*$ desc="", double low=DBL_MAX, double high=-DBL_MAX)

- virtual void computeMinMax (const char $*$, double \&min, double \&max) const 
- virtual void computeMinMax (const char $*$ dir)

- virtual void computeMinMax ()

- int dump (std::ostream \&out, size_t i) const

- virtual long evaluateRange (const ibis::qContinuousRange \&cmp, const ibis::bitvector \&mask, ibis::bitvector \&res) const

- $\operatorname{void} *$ getArray () const

- template $<$ typename $\mathrm{T}>$ int getRawData (array_t $<\mathrm{T}>$ \&vals) const

Note:

NO type check, caller need to make sure the currect type is specified.

- virtual ibis::fileManager::storage $*$ getRawData () const

- int limit (size_t nr)

- void reverseRows ()

- virtual array_t $\mathrm{t}<$ double $>*$ selectDoubles (const ibis::bitvector \&mask) const

Put the selected values into an array as doubles.

- virtual array_t $<$ float $>*$ selectFloats (const ibis::bitvector \&mask) const

Put selected values of a float column into an array.

- virtual array_t $<$ int32_t $>*$ selectInts (const ibis::bitvector \&mask) const

- virtual array_t $<$ int64_t $>*$ selectLongs (const ibis::bitvector \&mask) const

Can be called on all integral types.

- virtual array_t $<$ uint32_t $>*$ selectUInts (const ibis::bitvector \&mask) const

Can be called on columns of unsigned integral types, UINT, CATEGORY, USHORT, and UBYTE.

\section{Protected Member Functions}

- const column \& operator $=($ const column \& $)$

\section{Protected Attributes}

- void $*$ buffer

\subsubsection{Detailed Description}

An in-memory version of ibis::column.

The void $*$ points to an array $<\mathrm{T}>$ object where the type $\mathrm{T}$ is designated by column type.

\subsubsection{Member Function Documentation}

3.18.2.1 array_t $<$ double $>*$ ibis::bord::column::selectDoubles (const ibis::bitvector \& mask) const [virtual]

Put the selected values into an array as doubles.

Note:

Any column type could be selected as doubles. Other selectXXXs function only work on the same data type. This is the only function that allows one to convert to a different type. This is mainly to 
3.18.2.2 array_t $<$ int64_t $>*$ ibis::bord::column::selectLongs (const ibis::bitvector \& mask) const [virtual]

Can be called on all integral types.

Note that 64-byte unsigned integers are simply treated as signed integer. This may cause the values to be interperted incorrectly. Shorter version of unsigned integers are treated correctly as positive values.

The documentation for this class was generated from the following files:

- bord.h

- bord.cpp

\subsection{9 ibis::bundle Class Reference}

The public interface of bundles.

\#include <bundle.h>

Inheritance diagram for ibis::bundle::

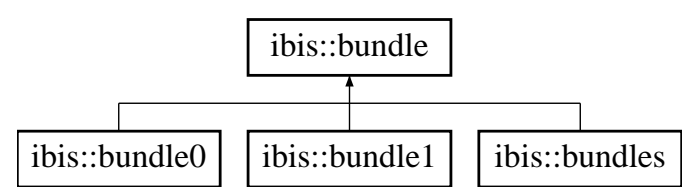

\section{Public Member Functions}

- virtual void $*$ columnArray (uint32_t j) const

Return the pointer to the underlying array used to store the jth column of the bundle.

- virtual ibis::TYPE_T columnType (uint32_t j) const

Return the type used to store the values of the jth column of the bundle.

- const ibis::RIDSet $*$ getRIDs () const

Return the pointer to all RIDs.

- const ibis::RIDSet $*$ getRIDs (uint32_t ind) const

Return the RIDs of the indth bundle.

- uint32_t numRowsInBundle (uint32_t ind) const

Compute the number of rows in bundle ind.

- virtual void print (std::ostream \&out) const=0

Print the bundle values to the specified output stream.

- virtual void printAll (std::ostream \&out) const=0

Print the bundle values along with the RIDs.

- virtual void reorder (const char $*$ names, int direction $)=0$

Re-order the bundles according to the new keys.

- uint32_t rowCounts (array_t $<$ uint32_t $>$ \&cnt) const

Compute the number of rows in each group(bundle). 
- virtual uint32_t size () const

Return the number of bundles.

- void sortRIDs (uint32_t i, uint32_t j)

- void swapRIDs (uint32_t $\mathrm{i}$, uint32_t j)

- virtual long truncate (const char $*$ names, int direction, uint32_t keep) $=0$

Truncate the list of bundle based on specified keys.

- virtual long truncate (uint32_t keep) $=0$

Truncate the list of bundles.

- virtual uint32_t width () const

Return the width of the bundles.

- virtual void write (const ibis::query $\&$ q) const $=0$

Write the bundle to the directory for the query $\mathrm{q}$.

- virtual double getDouble (uint32_t, uint32_t) const

Return the maximum double value.

- virtual float getFloat (uint32_t, uint32_t) const

Return the maximal float value.

- virtual int32_t getInt (uint32_t, uint32_t) const

Retrieve a specific value.

- virtual int64_t getLong (uint32_t, uint32_t) const

Return the maximal int value.

- virtual std::string getString (uint32_t, uint32_t) const

Retrieve a string value.

- virtual uint32_t getUInt (uint32_t, uint32_t) const

Return the maximal unsigned int value.

- virtual uint64_t getULong (uint32_t, uint32_t) const

Return the maximal unsigned int value.

\section{Static Public Member Functions}

- static bundle $*$ create (const ibis::part \&, const ibis::selected \&sel, const std::vector $<$ void $*>\&$ vals)

- static bundle $*$ create (const ibis::query \&q, const ibis::bitvector \&hits)

Create new bundle from a hit vector. Write info to q.dir().

- static bundle $*$ create (const ibis::query \&q)

Create a new bundle from previously stored information.

- static const ibis::RIDSet $*$ readRIDs (const char $*$ dir, const uint32_t i)

Return the RIDs related to the ith bundle. 


\section{Protected Member Functions}

- bundle (const ibis::query \&q, const ibis::bitvector \&hits)

- bundle (const ibis::query \&q)

- bundle (const ibis::selected \&c)

\section{Protected Attributes}

- const ibis::selected \& comps

- const char $*$ id

- bool infile

- ibis::RIDSet $*$ rids

- $\operatorname{array} \mathrm{t}<$ uint32_t $>*$ starts

\subsubsection{Detailed Description}

The public interface of bundles.

\subsubsection{Member Function Documentation}

\subsubsection{1 int32_t ibis::bundle::getInt (uint32_t, uint32_t) const [virtual]}

Retrieve a specific value.

Numerical values will be casted into the return type.

Note:

Most compilers will emit numerous complains about the potential data loss due to type conversions. User should employ the correct types to avoid actual loss of precision.

Reimplemented in ibis::bundle1, and ibis::bundles.

\subsubsection{2 std::string ibis::bundle::getString (uint32_t, uint32_t) const [virtual]}

Retrieve a string value.

It converts any data type to its string representation through the string stream library.

\section{Note:}

This is generic, but slow!

Reimplemented in ibis::bundle1, and ibis::bundles.

\subsubsection{3 uint32_t ibis::bundle::rowCounts (array_t $<$ uint32_t $>\&$ cnt) const}

Compute the number of rows in each group(bundle).

Return the number of bundles.

The documentation for this class was generated from the following files:

- bundle.h

- bundle.cpp 


\subsection{0 ibis::bundle0 Class Reference}

The null bundle. It contains only a list of RIDs.

\#include <bundle.h>

Inheritance diagram for ibis::bundle0::

\begin{tabular}{|l|}
\hline ibis::bundle \\
\hline ibis::bundle0 \\
\hline
\end{tabular}

\section{Public Member Functions}

- bundle0 (const ibis::query \&q, const ibis::bitvector \&hits)

- bundle0 (const ibis::query \&q)

- virtual void print (std::ostream \&out) const

Print the bundle values to the specified output stream.

- virtual void printAll (std::ostream \&out) const

Print the bundle values along with the RIDs.

- virtual void reorder (const char $*$ names, int direction)

Re-order the bundles according to the new keys.

- virtual uint32_t size () const

Return the number of bundles.

- virtual long truncate (const char $*$ names, int direction, uint32_t keep)

Truncate the list of bundle based on specified keys.

- virtual long truncate (uint32_t keep)

Truncate the list of bundles.

- virtual void write (const ibis::query \&q) const

Write the bundle to the directory for the query $\mathrm{q}$.

\subsubsection{Detailed Description}

The null bundle. It contains only a list of RIDs.

The documentation for this class was generated from the following files:

- bundle.h

- bundle.cpp

\subsection{1 ibis::bundle1 Class Reference}

The bundle with only one component.

\#include <bundle.h> 
Inheritance diagram for ibis::bundle1::

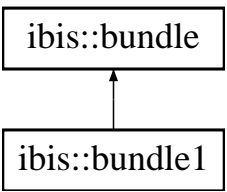

\section{Public Member Functions}

- bundle1 (const ibis::part \&tbl, const ibis::selected \&sel, const std::vector $<$ void $*>$ \&vals)

- bundle1 (const ibis::query \&q, const ibis::bitvector \&hits)

- bundle1 (const ibis::query \&q)

- virtual void $*$ columnArray (uint32_t $\mathrm{j}$ ) const

Return the pointer to the underlying array used to store the jth column of the bundle.

- virtual ibis::TYPE_T columnType (uint32_t j) const

Return the type used to store the values of the jth column of the bundle.

- virtual double getDouble (uint32_t, uint32_t) const

Return the maximal value defined in the class numeric_limits.

- virtual float getFloat (uint32_t, uint32_t) const

Return the maximal value defined in the class numeric_limits.

- virtual int32_t getInt (uint32_t, uint32_t) const

Return the maximal value defined in the class numeric_limits.

- virtual int64_t getLong (uint32_t, uint32_t) const

Return the maximal value defined in the class numeric_limits.

- virtual std::string getString (uint32_t, uint32_t) const

Convert any value to its string representation through std: : ostringstream.

- virtual uint32_t getUInt (uint32_t, uint32_t) const

Return the maximal value defined in the class numeric_limits.

- virtual uint64_t getULong (uint32_t, uint32_t) const

Return the maximal value defined in the class numeric_limits.

- virtual void print (std::ostream \&out) const

Print the bundle values to the specified output stream.

- virtual void printAll (std::ostream \&out) const

Print the bundle values along with the RIDs.

- virtual void reorder (const char $*$ names, int direction)

Re-order the bundles according to the new keys.

- virtual uint32_t size () const

Return the number of bundles. 
- virtual long truncate (const char $*$ names, int direction, uint32_t keep)

Truncate the list of bundle based on specified keys.

- virtual long truncate (uint32_t keep)

Truncate the list of bundles.

- virtual uint32_t width () const

Return the width of the bundles.

- virtual void write (const ibis::query \&) const

Write the bundle to the directory for the query $\mathrm{q}$.

\subsubsection{Detailed Description}

The bundle with only one component.

The documentation for this class was generated from the following files:

- bundle.h

- bundle.cpp

\subsection{2 ibis::bundles Class Reference}

The bundle with multiple components.

\#include <bundle.h>

Inheritance diagram for ibis::bundles::

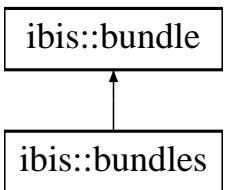

\section{Public Member Functions}

- bundles (const ibis::part \&tbl, const ibis::selected \&sel, const std::vector $<$ void $*>$ \&vals)

- bundles (const ibis::query \&q, const ibis::bitvector \&hits)

- bundles (const ibis::query \&q)

- virtual void $*$ columnArray (uint32_t j) const

Return the pointer to the underlying array used to store the jth column of the bundle.

- virtual ibis::TYPE_T columnType (uint32_t j) const

Return the type used to store the values of the jth column of the bundle.

- virtual double getDouble (uint32_t, uint32_t) const

Return the maximal value defined in the class numeric_limits.

- virtual float getFloat (uint32_t, uint32_t) const

Return the maximal value defined in the class numeric_limits. 
- virtual int32_t getInt (uint32_t, uint32_t) const

Return the maximal value defined in the class numeric_limits.

- virtual int64_t getLong (uint32_t, uint32_t) const

Return the maximal value defined in the class numeric_limits.

- virtual std::string getString (uint32_t, uint32_t) const

Convert any value to its string representation through std: : ostringstream.

- virtual uint32_t getUInt (uint32_t, uint32_t) const

Return the maximal value defined in the class numeric_limits.

- virtual uint64_t getULong (uint32_t, uint32_t) const

Return the maximal value defined in the class numeric_limits.

- virtual void print (std::ostream \&out) const

Print the bundle values to the specified output stream.

- virtual void printAll (std::ostream \&out) const

Print the bundle values along with the RIDs.

- virtual void reorder (const char $*$ names, int direction)

Reorder the bundles according to the keys (names) given.

- virtual uint32_t size () const

Return the number of bundles.

- virtual long truncate (const char $*$ names, int direction, uint32_t keep)

Reorder the bundles according to the keys (names) given.

- virtual long truncate (uint32_t keep)

Truncate the list of bundles.

- virtual uint32_t width () const

Return the width of the bundles.

- virtual void write (const ibis::query $\&$ ) const

Write the bundle to the directory for the query $\mathrm{q}$.

\subsubsection{Detailed Description}

The bundle with multiple components.

\subsubsection{Member Function Documentation}

\subsubsection{1 void ibis::bundles::reorder (const char $*$ names, int direction) [ virtual]}

Reorder the bundles according to the keys (names) given.

turn counts back into starting positions (starts)

Implements ibis::bundle. 


\subsubsection{2 long ibis::bundles::truncate (const char $*$ names, int direction, uint32_t keep) [ virtual]}

Reorder the bundles according to the keys (names) given.

Keep only the first keep elements. If direction $<0$, keep the largest ones, otherwise keep the smallest ones. turn counts back into starting positions (starts)

Implements ibis::bundle.

The documentation for this class was generated from the following files:

- bundle.h

- bundle.cpp

\subsection{3 ibis::bylt Class Reference}

The two-level range-equality code.

\#include <irelic.h>

Inheritance diagram for ibis::bylt::

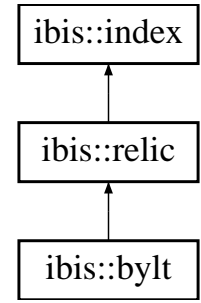

\section{Public Member Functions}

- virtual long append (const char $* \mathrm{dt}$, const char $* \mathrm{df}$, uint32_t nnew)

Extend the index.

- bylt (const ibis::column $*$ c, ibis::fileManager::storage $*$ st, uint32_t offset=8)

The leading portion of the index file is the same as ibis::relic, which allows the constructor of the base class to work properly.

- bylt (const ibis::column $* \mathrm{c}=0$, const char $* \mathrm{f}=0$ )

- virtual uint32_t estimate (const ibis::qContinuousRange \&expr) const Returns an upper bound on the number of hits.

- virtual double estimateCost (const ibis::qContinuousRange \&expr) const Estimate the code of evaluate a range condition.

- virtual long evaluate (const ibis::qContinuousRange \&expr, ibis::bitvector \&hits) const To evaluate the exact hits.

- virtual const char $*$ name () const

Returns the name of the index, similar to the function type, but returns a string instead.

- virtual void print (std::ostream \&out) const

Prints human readable information. 
- virtual void read (ibis::fileManager::storage $*$ st)

Reconstructs an index from an array of bytes.

- virtual void read (const char $*$ idxfile)

Reconstructs an index from the named file.

- virtual INDEX_TYPE type () const

Returns an index type identifier.

- virtual void write (const char $* \mathrm{dt})$ const

Save index to a file.

\section{Protected Member Functions \\ - virtual void clear () \\ Clear the existing content.}

\subsubsection{Detailed Description}

The two-level range-equality code.

\section{Note:}

Bylt is Danish word for pack, the name of the binned version of the two-level range-equality code.

\subsubsection{Constructor \& Destructor Documentation}

\subsubsection{1 ibis::bylt::bylt (const ibis::column $* \boldsymbol{c}$, ibis::fileManager::storage $* s t$, uint32_t start $=8$ )}

The leading portion of the index file is the same as ibis::relic, which allows the constructor of the base class to work properly.

The content following the last bitvector in ibis::relic is as follows, writeCoarse.

nc (uint32_t) - number of coarse bins. cbounds (unsigned[nc+1]) - boundaries of the coarse bins. coffsets(int32_$\mathrm{t}[\mathrm{nc}+1]$ ) - starting position of the coarse level bitmaps. cbits (bitvector[nc]) - bitvector laid out one after another.

\subsubsection{Member Function Documentation}

3.23.3.1 long ibis::bylt::evaluate (const ibis::qContinuousRange \& expr, ibis::bitvector \& hits) const [virtual]

To evaluate the exact hits.

On success, return the number of hits, otherwise a negative value is returned.

Reimplemented from ibis::relic.

\subsubsection{2 void ibis::bylt::print (std::ostream \& out) const [virtual]}

Prints human readable information.

Outputs information about the index as text to the specified output stream.

Reimplemented from ibis::relic. 


\subsubsection{3 void ibis::bylt::read (ibis::fileManager::storage $* s t$ ) [virtual]}

Reconstructs an index from an array of bytes.

Intended for internal use only!

Reimplemented from ibis::relic.

\subsubsection{4 void ibis::bylt::read (const char $*$ idxfile) $\quad$ [virtual]}

Reconstructs an index from the named file.

The name can be the directory containing an index file. In this case, the name of the index file must be the name of the column followed by ".idx" suffix.

Reimplemented from ibis::relic.

\subsubsection{5 void ibis::bylt::write (const char $* \boldsymbol{d} t)$ const [virtual]}

Save index to a file.

Outputs the index in a compact binary format to the named file or directory. The index file contains a header that can be identified by the function isIndex.

Reimplemented from ibis::relic.

The documentation for this class was generated from the following files:

- irelic.h

- ixbylt.cpp

\subsection{4 ibis::category Class Reference}

A specialized low-cardinality text field.

\#include <category.h>

Inheritance diagram for ibis::category::

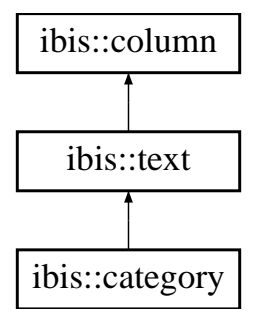

\section{Public Member Functions}

- virtual long append (const char $* \mathrm{dt}$, const char $* \mathrm{df}$, const uint32_t nold, const uint32_t nnew, const uint32_t nbuf, char $*$ buf)

Append the content in $\mathrm{df}$ to the directory $\mathrm{dt}$.

- category (const part $* \mathrm{tbl}$, const char $*$ name, const char $*$ value, const char $*$ dir $=0$, uint $32 \_t$ nevt $=0$ )

- category (const ibis::column \&col)

Copy constructor. Copy from a collumn object with KEY type.

- category (const part $*$ tbl, const char $*$ name)

- category (const part *tbl, FILE $*$ file) 
- virtual double estimateCost (const ibis::qMultiString \& cmp) const

- virtual double estimateCost (const ibis::qString \&cmp) const

- virtual const char $*$ getKey (uint32_t i) const

Return the ith value in the dictionary.

- virtual const char $*$ isKey (const char $*$ str) const

Is the given string one of the keys in the dictionary? Return a null pointer if not.

- virtual void print (std::ostream \&out) const

- virtual long search (const std::vector $<$ std::string $>\&$ vals) const

Estimate the total number of matches for a list of strings.

- virtual long search (const char $*$ str) const

Estimate the number of matches.

- virtual long search (const std::vector $<$ std::string $>$ \&vals, ibis::bitvector \&hits) const

Match a list of strings.

- virtual long search (const char $*$ str, ibis::bitvector \&hits) const

Match a particular string.

- virtual array_t $<$ uint32_t $>*$ selectUInts (const bitvector \&mask) const

Return the integer values of the records marked 1 in the mask.

- virtual void write (FILE $*$ file) const

Write the current content to the TDC file.

\subsubsection{Detailed Description}

A specialized low-cardinality text field.

It is also known as control values, or categorical values. This implementation directly converts string values into bitvectors (as ibis::relic), and does not store integer version of the string.

Note:

Value zero (0) is reserved for NULL values.

The documentation for this class was generated from the following files:

- category.h

- category.cpp

\subsection{5 ibis::colDoubles Class Reference}

A class to store double precision floating-point values.

\#include <colvalues.h>

Inheritance diagram for ibis::colDoubles::

\begin{tabular}{|l|}
\hline ibis::colValues \\
\hline ibis::colDoubles \\
\hline
\end{tabular}




\section{Public Member Functions}

- virtual void bottomk (uint32_t $\mathrm{k}$, array_t $<$ uint32_t $>$ \&ind) const

Return the positions of the $\mathrm{k}$ smallest elements.

- colDoubles (const ibis::column $* \mathrm{c}$, void $*$ vals)

- colDoubles (const ibis::column $*$ c, ibis::fileManager::storage $*$ store, const uint32_t start, const uint32_t nelm)

- colDoubles (const ibis::column $*$ c, const ibis::bitvector \&hits)

- virtual uint32_t elementSize () const

- virtual bool empty () const

- virtual void erase (uint32_t i, uint32_t j)

- virtual void $*$ getArray () const

Return the pointer to the pointer to underlying array_t $t<T>$ object.

- virtual double getDouble (uint32_t i) const

- virtual float getFloat (uint32_t i) const

- virtual int32_t getInt (uint32_t i) const

- virtual int64_t getLong (uint32_t i) const

- virtual double getMax () const

- virtual double getMin () const

- virtual double getSum () const

- virtual ibis::TYPE_T getType () const

Return the type of the data stored.

- virtual uint32_t getUInt (uint32_t i) const

- virtual uint64_t getULong (uint32_t i) const

- virtual const ibis::column $*$ operator $\rightarrow$ () const

Provide a pointer to the column containing the selected values.

- virtual void reduce (const array_t $<$ uint32_t $>$ \&starts, ibis::selected::FUNCTION func)

- virtual void reduce (const array_t $<$ uint32_t $>$ \&starts)

- virtual void reorder (const array_t $<$ uint32_t $>$ \&ind)

Reorder the values according to the specified indices.

- virtual array_t $<$ uint32_t $>*$ segment (const array_t $<$ uint32_t $>*$ old $=0$ ) const

Produce an array of the starting positions of values that are the same.

- virtual uint32_t size () const

- virtual void sort (uint32_t i, uint32_t j, array_t $<$ uint32_t $>$ \&neworder) const

Sort rows in the range $[i, j)$.

- virtual void sort (uint32_t i, uint32_t j, bundle $*$ bdl, colList::iterator head, colList::iterator tail)

Sort rows in the range $[i, j)$.

- virtual void sort (uint32_t i, uint32_t j, bundle $*$ bdl)

Sort rows in the range $[i, j)$.

- void swap (colDoubles \&rhs)

- virtual void swap (uint32_t i, uint32_t j)

- virtual void topk (uint32_t k, array_t $<$ uint32_t $>$ \&ind) const

Return the positions of the $\mathrm{k}$ largest elements.

- virtual long truncate (uint32_t keep) 
Truncate the number element to no more than keep.

- virtual void write (std::ostream \&out, uint32_t i) const

Write ith element as text.

- virtual uint32_t write (FILE $*$ fptr) const

Write out whole array as binary.

\subsubsection{Detailed Description}

A class to store double precision floating-point values.

\subsubsection{Member Function Documentation}

3.25.2.1 virtual void ibis::colDoubles::reorder (const array_t $\mathbf{t}<$ uint32_t $>\boldsymbol{\&}$ ind) $\quad$ inline, virtual]

Reorder the values according to the specified indices.

New[i] $=$ Old[ind[i]].

Implements ibis::colValues.

3.25.2.2 void ibis::colDoubles::sort (uint32_t $i$, uint32_t $j$, array_t $<$ uint32_t $>\&$ neworder) const [virtual]

Sort rows in the range $[i, j)$.

Output the new order in array neworder.

Implements ibis::colValues.

3.25.2.3 void ibis::colDoubles::sort (uint32_t $i$, uint32_t $j$, bundle $* b d l$, colList::iterator head, colList::iterator tail) [virtual]

Sort rows in the range $[i, j)$.

Also sort the columns between [head, tail).

Implements ibis::colValues.

The documentation for this class was generated from the following files:

- colValues.h

- colValues.cpp

\subsection{6 ibis::colFloats Class Reference}

A class to store single precision float-point values.

\#include <colvalues.h>

Inheritance diagram for ibis::colFloats:: 
ibis::colValues

ibis::colFloats

\section{Public Member Functions}

- virtual void bottomk (uint32_t k, array_t $<$ uint32_t $>$ \&ind) const

Return the positions of the $\mathrm{k}$ smallest elements.

- colFloats (const ibis::column $*$ c, void $*$ vals)

- colFloats (const ibis::column $* \mathrm{c}$, ibis::fileManager::storage $*$ store, const uint32_t start, const uint32_t nelm)

- colFloats (const ibis::column $*$ c, const ibis::bitvector \&hits)

- virtual uint32_t elementSize () const

- virtual bool empty () const

- virtual void erase (uint32_t i, uint32_t j)

- virtual void $*$ getArray () const

Return the pointer to the pointer to underlying array_t $<T>$ object.

- virtual double getDouble (uint32_t i) const

- virtual float getFloat (uint32_t i) const

- virtual int32_t getInt (uint32_t i) const

- virtual int64_t getLong (uint32_t i) const

- virtual double getMax () const

- virtual double getMin () const

- virtual double getSum () const

- virtual ibis::TYPE_T getType () const

Return the type of the data stored.

- virtual uint32_t getUInt (uint32_t i) const

- virtual uint64_t getULong (uint32_t i) const

- virtual const ibis::column $*$ operator $\rightarrow$ () const

Provide a pointer to the column containing the selected values.

- virtual void reduce (const array_t $<$ uint32_t $>$ \&starts, ibis::selected::FUNCTION func)

- virtual void reduce (const array_t $<$ uint32_t $>$ \&starts)

- virtual void reorder (const array_t $<$ uint32_t $>$ \&ind)

Reorder the values according to the specified indices.

- virtual array_t $<$ uint32_t $>*$ segment (const array_t $<$ uint32_t $>*$ old $=0$ ) const

Produce an array of the starting positions of values that are the same.

- virtual uint32_t size () const

- virtual void sort (uint32_t i, uint32_t j, array_t $<$ uint32_t $>$ \&neworder) const

Sort rows in the range $[i, j)$.

- virtual void sort (uint32_t i, uint32_t j, bundle $*$ bdl, colList::iterator head, colList::iterator tail)

Sort rows in the range $[i, j)$.

- virtual void sort (uint32_t i, uint32_t j, bundle $*$ bdl)

Sort rows in the range $[i, j)$. 
- void swap (colFloats \&rhs)

- virtual void swap (uint32_t i, uint32_t j)

- virtual void topk (uint32_t k, array_t $<$ uint32_t $>$ \&ind) const

Return the positions of the $\mathrm{k}$ largest elements.

- virtual long truncate (uint32_t keep)

Truncate the number element to no more than keep.

- virtual void write (std::ostream \&out, uint32_t i) const

Write ith element as text.

- virtual uint32_t write (FILE $*$ fptr) const

Write out whole array as binary.

\subsubsection{Detailed Description}

A class to store single precision float-point values.

\subsubsection{Member Function Documentation}

3.26.2.1 virtual void ibis::colFloats::reorder (const array_t $<$ uint32_t $>\boldsymbol{\&}$ ind) [inline, virtual ] Reorder the values according to the specified indices.

New $[i]=$ Old [ind [i]].

Implements ibis::colValues.

3.26.2.2 void ibis::colFloats::sort (uint32_t $\boldsymbol{i}$, uint32_t $j$, array_t $<$ uint32_t $>$ \& neworder) const [ virt $u a$ l ]

Sort rows in the range $[i, j)$.

Output the new order in array neworder.

Implements ibis::colValues.

3.26.2.3 void ibis::colFloats::sort (uint32_t $i$, uint32_t $j$, bundle $* b d l$, colList::iterator head, colList::iterator tail) [virtual]

Sort rows in the range $[i, j)$.

Also sort the columns between [head, tail).

Implements ibis::colValues.

The documentation for this class was generated from the following files:

- colValues.h

- colValues.cpp

\subsection{7 ibis::colInts Class Reference}

A class to store integer values.

\#include <colvalues.h> 
Inheritance diagram for ibis::colInts::

ibis::colValues

ibis::colInts

\section{Public Member Functions}

- virtual void bottomk (uint32_t $\mathrm{k}$, array_t $<$ uint32_t $>$ \&ind) const

Return the positions of the $\mathrm{k}$ smallest elements.

- colInts (const ibis::column $* \mathrm{c}$, void $*$ vals)

- colInts (const ibis::column $*$ c, ibis::fileManager::storage $*$ store, const uint32_t start, const uint32_t nelm)

- colInts (const ibis::column $*$ c, const ibis::bitvector \&hits)

- virtual uint32_t elementSize () const

- virtual bool empty () const

- virtual void erase (uint32_t $i$, uint32_t j)

- virtual void $*$ getArray () const

Return the pointer to the pointer to underlying array_t $<T>$ object.

- virtual double getDouble (uint32_t i) const

- virtual float getFloat (uint32_t i) const

- virtual int32_t getInt (uint32_t i) const

- virtual int64_t getLong (uint32_t i) const

- virtual double getMax () const

- virtual double getMin () const

- virtual double getSum () const

- virtual ibis::TYPE_T getType () const

Return the type of the data stored.

- virtual uint32_t getUInt (uint32_t i) const

- virtual uint64_t getULong (uint32_t i) const

- virtual const ibis::column $*$ operator $\rightarrow()$ const

Provide a pointer to the column containing the selected values.

- virtual void reduce (const array_t $<$ uint32_t $>$ \&starts, ibis::selected::FUNCTION func)

- virtual void reduce (const array_t $<$ uint32_t $>$ \&starts)

- virtual void reorder (const array_t $<$ uint32_t $>$ \&ind)

Reorder the values according to the specified indices.

- virtual array_t $\mathrm{t}<$ uint32_t $>*$ segment (const array_t $\mathrm{t}<$ uint32_t $>*$ old $=0$ ) const

Produce an array of the starting positions of values that are the same.

- virtual uint32_t size () const

- virtual void sort (uint32_t $\mathrm{i}$, uint32_t $\mathrm{j}$, array_t $<$ uint32_t $>$ \&neworder) const

Sort rows in the range $[i, j)$.

- virtual void sort (uint32_t i, uint32_t j, bundle $*$ bdl, colList::iterator head, colList::iterator tail)

Sort rows in the range $[i, j)$. 
- virtual void sort (uint32_t $\mathrm{i}$, uint32_t j, bundle $*$ bdl)

Sort rows in the range $[i, j)$.

- void swap (colInts \&rhs)

- virtual void swap (uint32_t i, uint32_t j)

- virtual void topk (uint32_t $\mathrm{k}$, array_t $<$ uint32_t $>$ \&ind) const Return the positions of the $\mathrm{k}$ largest elements.

- virtual long truncate (uint32_t keep)

Truncate the number element to no more than keep.

- virtual void write (std::ostream \&out, uint32_t i) const

Write ith element as text.

- virtual uint32_t write (FILE $*$ fptr) const

Write out whole array as binary.

\subsubsection{Detailed Description}

A class to store integer values.

\subsubsection{Member Function Documentation}

3.27.2.1 virtual void ibis::colInts::reorder (const array_t $<$ uint32_t $>$ \& ind) [inline, virtual]

Reorder the values according to the specified indices.

New[i] $=$ Old[ind[i] $]$.

Implements ibis::colValues.

3.27.2.2 void ibis::colInts::sort (uint32_t $\boldsymbol{i}$, uint32_t $j$, array_t $<$ uint32_t $>\&$ neworder) const [virtual]

Sort rows in the range $[i, j)$.

Output the new order in array neworder.

Implements ibis::colValues.

3.27.2.3 void ibis::colInts::sort (uint32_t $i$, uint32_t $j$, bundle $* b d l$, colList::iterator head, colList::iterator tail) [virtual]

Sort rows in the range $[i, j)$.

Also sort the columns between [head, tail).

Implements ibis::colValues.

The documentation for this class was generated from the following files:

- colValues.h

- colValues.cpp 


\subsection{8 ibis::colLongs Class Reference}

A class to store integer values.

\#include <colvalues.h>

Inheritance diagram for ibis::colLongs::

$$
\begin{array}{|l|}
\hline \text { ibis::colValues } \\
\hline \text { ibis::colLongs } \\
\hline
\end{array}
$$

\section{Public Member Functions}

- virtual void bottomk (uint32_t k, array_t $<$ uint32_t $>$ \&ind) const Return the positions of the $\mathrm{k}$ smallest elements.

- colLongs (const ibis::column $*$ c, void $*$ vals)

- colLongs (const ibis::column $* \mathrm{c}$, ibis::fileManager::storage $*$ store, const uint32_t start, const uint32_t nelm)

- colLongs (const ibis::column $*$ c, const ibis::bitvector \&hits)

- virtual uint32_t elementSize () const

- virtual bool empty () const

- virtual void erase (uint32_t i, uint32_t j)

- virtual void $*$ getArray () const

Return the pointer to the pointer to underlying array_t $<T>$ object.

- virtual double getDouble (uint32_t i) const

- virtual float getFloat (uint32_t i) const

- virtual int32_t getInt (uint32_t i) const

- virtual int64_t getLong (uint32_t i) const

- virtual double getMax () const

- virtual double getMin () const

- virtual double getSum () const

- virtual ibis::TYPE_T getType () const

Return the type of the data stored.

- virtual uint32_t getUInt (uint32_t i) const

- virtual uint64_t getULong (uint32_t i) const

- virtual const ibis::column $*$ operator $\rightarrow$ () const

Provide a pointer to the column containing the selected values.

- virtual void reduce (const array_t $<$ uint32_t $>\&$ starts, ibis::selected::FUNCTION func)

- virtual void reduce (const array_t $<$ uint32_t $>$ \&starts)

- virtual void reorder (const array_t $\mathrm{t}<$ uint32_t $>$ \&ind)

Reorder the values according to the specified indices.

- virtual array_t $\mathrm{t}<$ uint32_t $>*$ segment (const array_t $\mathrm{t}<$ uint32_t $>*$ old $=0$ ) const

Produce an array of the starting positions of values that are the same.

- virtual uint32_t size () const

- virtual void sort (uint32_t $\mathrm{i}$, uint32_t $\mathrm{j}$, array_t $<$ uint32_t $>$ \&neworder) const

Sort rows in the range $[i, j)$. 
- virtual void sort (uint32_t i, uint32_t j, bundle $*$ bdl, colList::iterator head, colList::iterator tail) Sort rows in the range $[i, j)$.

- virtual void sort (uint32_t i, uint32_t j, bundle $*$ bdl)

Sort rows in the range $[i, j)$.

- void swap (colLongs \&rhs)

- virtual void swap (uint32_t i, uint32_t j)

- virtual void topk (uint32_t $\mathrm{k}$, array_t $<$ uint32_t $>$ \&ind) const Return the positions of the $\mathrm{k}$ largest elements.

- virtual long truncate (uint32_t keep)

Truncate the number element to no more than keep.

- virtual void write (std::ostream \&out, uint32_t i) const

Write ith element as text.

- virtual uint32_t write (FILE $*$ fptr) const

Write out whole array as binary.

\subsubsection{Detailed Description}

A class to store integer values.

\subsubsection{Member Function Documentation}

3.28.2.1 virtual void ibis::colLongs::reorder (const array_t $\mathbf{t}<\mathbf{u i n t 3 2 \_ t}>\boldsymbol{\&}$ ind) $\quad$ [ inline, virtual] Reorder the values according to the specified indices.

New $[i]=$ Old[ind[i] $]$.

Implements ibis::colValues.

3.28.2.2 void ibis::colLongs::sort (uint32_t $i$, uint32_t $j$, array_t $\mathbf{t}<$ uint32_t $>$ \& neworder) const [virtual]

Sort rows in the range $[i, j)$.

Output the new order in array neworder.

Implements ibis::colValues.

3.28.2.3 void ibis::colLongs::sort (uint32_t $i$, uint32_t $j$, bundle $* b d l$, colList::iterator head, colList::iterator tail) [virtual]

Sort rows in the range $[i, j)$.

Also sort the columns between [head, tail).

Implements ibis::colValues.

The documentation for this class was generated from the following files:

- colValues.h

- colValues.cpp 


\subsection{9 ibis::colUInts Class Reference}

A class to store unsigned integer values.

\#include <colvalues.h>

Inheritance diagram for ibis::colUInts::

$$
\begin{array}{|l|}
\hline \text { ibis::colValues } \\
\hline \text { ibis::colUInts } \\
\hline
\end{array}
$$

\section{Public Member Functions}

- virtual void bottomk (uint32_t k, array_t $<$ uint32_t $>$ \&ind) const Return the positions of the $\mathrm{k}$ smallest elements.

- colUInts (const ibis::column $* \mathrm{c}$, void $*$ vals)

- colUInts (const ibis::column $*$ c, ibis::fileManager::storage $*$ store, const uint32_t start, const uint32_t nelm)

- colUInts (const ibis::column $*$ c, const ibis::bitvector \&hits)

- virtual uint32_t elementSize () const

- virtual bool empty () const

- virtual void erase (uint32_t i, uint32_t j)

- virtual void $*$ getArray () const

Return the pointer to the pointer to underlying array_t $<T>$ object.

- virtual double getDouble (uint32_t i) const

- virtual float getFloat (uint32_t i) const

- virtual int32_t getInt (uint32_t i) const

- virtual int64_t getLong (uint32_t i) const

- virtual double getMax () const

- virtual double getMin () const

- virtual double getSum () const

- virtual ibis::TYPE_T getType () const

Return the type of the data stored.

- virtual uint32_t getUInt (uint32_t i) const

- virtual uint64_t getULong (uint32_t i) const

- virtual const ibis::column $*$ operator $\rightarrow$ () const

Provide a pointer to the column containing the selected values.

- virtual void reduce (const array_t $<$ uint32_t $>$ \&starts, ibis::selected::FUNCTION func)

- virtual void reduce (const array_t $<$ uint32_t $>$ \&starts)

- virtual void reorder (const array_t $<$ uint32_t $>$ \&ind)

Reorder the values according to the specified indices.

- virtual array_t $<$ uint32_t $>*$ segment (const array_t $<$ uint32_t $>*$ old $=0$ ) const

Produce an array of the starting positions of values that are the same.

- virtual uint32_t size () const

- virtual void sort (uint32_t i, uint32_t j, array_t $<$ uint32_t $>$ \&neworder) const

Sort rows in the range $[i, j)$. 
- virtual void sort (uint32_t i, uint32_t j, bundle $*$ bdl, colList::iterator head, colList::iterator tail) Sort rows in the range $[i, j)$.

- virtual void sort (uint32_t i, uint32_t j, bundle $*$ bdl)

Sort rows in the range $[i, j)$.

- void swap (colUInts \& rhs)

- virtual void swap (uint32_t i, uint32_t j)

- virtual void topk (uint32_t $\mathrm{k}$, array_t $<$ uint32_t $>$ \&ind) const Return the positions of the $\mathrm{k}$ largest elements.

- virtual long truncate (uint32_t keep)

Truncate the number element to no more than keep.

- virtual void write (std::ostream \&out, uint32_t i) const

Write the ith element as text.

- virtual uint32_t write (FILE $*$ fptr) const

Write out the whole array as binary.

\subsubsection{Detailed Description}

A class to store unsigned integer values.

\subsubsection{Member Function Documentation}

\subsubsection{1 virtual void ibis::colUInts::reorder (const array_t $<$ uint32_t $>\boldsymbol{\&}$ ind) $\quad$ [inline, virtual ]}

Reorder the values according to the specified indices.

New[i] $=$ Old[ind[i]].

Implements ibis::colValues.

\subsubsection{2 void ibis::colUInts::sort (uint32_t $\boldsymbol{i}$, uint32_t $\boldsymbol{j}$, array_t $<$ uint32_t $>\boldsymbol{\&}$ neworder) const [ virtual]}

Sort rows in the range $[i, j)$.

Output the new order in array neworder.

Implements ibis::colValues.

3.29.2.3 void ibis::colUInts::sort (uint32_t $i$, uint32_t $j$, bundle $* b d l$, colList::iterator head, colList::iterator tail) [virtual]

Sort rows in the range $[i, j)$.

Also sort the columns between [head, tail).

Implements ibis::colValues.

The documentation for this class was generated from the following files:

- colValues.h

- colValues.cpp 


\subsection{0 ibis::colULongs Class Reference}

A class to store unsigned integer values.

\#include <colvalues.h>

Inheritance diagram for ibis::colULongs::

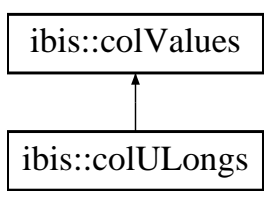

\section{Public Member Functions}

- virtual void bottomk (uint32_t $\mathrm{k}$, array_t $<$ uint32_t $>$ \&ind) const Return the positions of the $\mathrm{k}$ smallest elements.

- colULongs (const ibis::column $*$ c, void $*$ vals)

- colULongs (const ibis:: column $* \mathrm{c}$, ibis::fileManager::storage $*$ store, const uint32_t start, const uint32_t nelm)

- colULongs (const ibis::column $*$ c, const ibis::bitvector \&hits)

- virtual uint32_t elementSize () const

- virtual bool empty () const

- virtual void erase (uint32_t $i$, uint32_t j)

- virtual void $*$ getArray () const

Return the pointer to the pointer to underlying array_t $t<T>$ object.

- virtual double getDouble (uint32_t i) const

- virtual float getFloat (uint32_t i) const

- virtual int32_t getInt (uint32_t i) const

- virtual int64_t getLong (uint32_t i) const

- virtual double getMax () const

- virtual double getMin () const

- virtual double getSum () const

- virtual ibis::TYPE_T getType () const

Return the type of the data stored.

- virtual uint32_t getUInt (uint32_t i) const

- virtual uint64_t getULong (uint32_t i) const

- virtual const ibis::column $*$ operator $\rightarrow$ () const

Provide a pointer to the column containing the selected values.

- virtual void reduce (const array_t $<$ uint32_t $>$ \&starts, ibis::selected::FUNCTION func)

- virtual void reduce (const array_t $<$ uint32_t $>$ \&starts)

- virtual void reorder (const array_t $\mathrm{t}<$ uint32_t $>$ \&ind)

Reorder the values according to the specified indices.

- virtual array_t $\mathrm{t}<$ uint 32_t $>*$ segment (const array_t $\mathrm{t}<$ uint32_t $>*$ old $=0$ ) const

Produce an array of the starting positions of values that are the same.

- virtual uint32_t size () const

- virtual void sort (uint32_t i, uint32_t j, array_t $<$ uint32_t $>$ \&neworder) const

Sort rows in the range $[i, j)$. 
- virtual void sort (uint32_t i, uint32_t j, bundle $*$ bdl, colList::iterator head, colList::iterator tail) Sort rows in the range $[i, j)$.

- virtual void sort (uint32_t i, uint32_t j, bundle $*$ bdl)

Sort rows in the range $[i, j)$.

- void swap (colULongs \&rhs)

- virtual void swap (uint32_t i, uint32_t j)

- virtual void topk (uint32_t k, array_t $<$ uint32_t $>$ \&ind) const Return the positions of the $\mathrm{k}$ largest elements.

- virtual long truncate (uint32_t keep)

Truncate the number element to no more than keep.

- virtual void write (std::ostream \&out, uint32_t i) const

Write the ith element as text.

- virtual uint32_t write (FILE $* \mathrm{fptr})$ const

Write out the whole array as binary.

\subsubsection{Detailed Description}

A class to store unsigned integer values.

\subsubsection{Member Function Documentation}

3.30.2.1 virtual void ibis::colULongs::reorder (const array_t $<$ uint32_t $>$ \& ind) [inline, virtual ] Reorder the values according to the specified indices.

New $[i]=$ Old[ind [i]].

Implements ibis::colValues.

3.30.2.2 void ibis::colULongs::sort (uint32_t $i$, uint32_t $j$, array_t $<$ uint32_t $>$ \& neworder) const [virtual]

Sort rows in the range $[i, j)$.

Output the new order in array neworder.

Implements ibis::colValues.

3.30.2.3 void ibis::colULongs::sort (uint32_t $i$, uint32_t $j$, bundle $* b d l$, colList::iterator head, colList::iterator tail) [virtual]

Sort rows in the range $[i, j)$.

Also sort the columns between [head, tail).

Implements ibis::colValues.

The documentation for this class was generated from the following files:

- colValues.h

- colValues.cpp 


\subsection{1 ibis::column Class Reference}

The class to represent a column of a data table.

\#include <column.h>

Inheritance diagram for ibis::column::

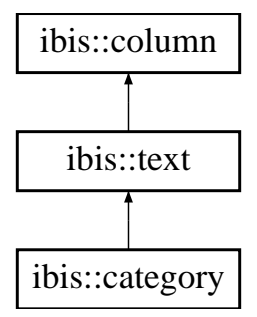

\section{Public Member Functions}

- virtual long append (const char $* \mathrm{dt}$, const char $* \mathrm{df}$, const uint32_t nold, const uint32_t nnew, const uint32_t nbuf, char $*$ buf)

Append new data in directory $d f$ to the end of existing data in $d t$.

- void binWeights (std::vector $<$ uint32_t $>\&$ ) const

- column (const column \&rhs)

copy constructor

- column (const part $*$ tbl, ibis::TYPE_T t, const char $*$ name, const char $*$ desc="", double low=DBL_MAX, double high=-DBL_MAX)

Construct a new column of specified type.

- column (const part $*$ tbl, FILE $*$ file)

Reconstitute a column from the content of a file.

- virtual void computeMinMax (const char $*$ dir, double \&min, double \&max) const

Compute the actual min/max of the data in directory dir.

- virtual void computeMinMax (const char $*$ dir)

- virtual void computeMinMax ()

Compute the actual min/max values by actually going through all the values.

- int contractRange (ibis::qContinuousRange \&rng) const

- char $*$ dataFileName (const char $* \operatorname{dir}=0$ ) const

Name of the data file in the given data directory.

- const part $*$ dataTable () const

- void description (const char $* \mathrm{~d}$ )

- const char $*$ description () const

- int elementSize () const

- virtual double estimateCost (const ibis::qMultiString \&cmp) const

- virtual double estimateCost (const ibis::qString \&cmp) const

- double estimateCost (const ibis::qDiscreteRange \&cmp) const

- double estimateCost (const ibis::qContinuousRange $\& \mathrm{cmp}$ ) const

Estimate the cost of evaluate the query expression. 
- long estimateRange (const ibis::qDiscreteRange \&cmp) const

- long estimateRange (const ibis::qContinuousRange \&cmp) const Use an index to compute an upper bound on the number of hits.

- long estimateRange (const ibis::qDiscreteRange \&cmp, ibis::bitvector \&low, ibis::bitvector \&high) const

- long estimateRange (const ibis::qContinuousRange \&cmp, ibis::bitvector \&low, ibis::bitvector \&high) const Compute a lower bound and an upper bound on the number of hits using the bitmap index.

- long evaluateRange (const ibis::qContinuousRange \&cmp, const ibis::bitvector \&mask, ibis::bitvector \&res) const

Attempt to compute the exact answer.

- int expandRange (ibis::qContinuousRange \&rng) const

expand/contract range condition so that the new ranges fall exactly on the bin boundaries

- virtual const char $*$ findString (const char $*$ str) const

Determine if the input string is one of the records.

- double getActualMax () const

Compute the actual maximum value by reading the data or examining the index.

- double getActualMin () const

Compute the actual minimum value by reading the data or examining the index.

- long getCumulativeDistribution (std::vector $<$ double $>$ \&bounds, std::vector $<$ uint32_t $>$ \&counts) const

Compute the actual data distribution.

- long getDistribution (std::vector $<$ double $>$ \&bbs, std::vector $<$ uint32_t $>$ \&counts) const

Count the number of records in each bin.

- array_t $<$ double $>*$ getDoubleArray () const

- array_t $<$ float $>*$ getFloatArray () const

- $\operatorname{array} \_\mathrm{t}<$ int32_t $>*$ getIntArray () const

Return all rows of the column as an array_t object.

- void getNullMask (bitvector \&mask) const

- template $<$ typename $\mathrm{T}>$ int getRawData $\left(\operatorname{array} \_\mathrm{t}<\mathrm{T}>\right.$ \&vals) const

- ibis::fileManager::storage $*$ getRawData () const

- virtual void getString (uint32_t i, std::string \&val) const

- virtual const char $*$ getString (uint32_t i) const

Return the internal string value for the integer.

- double getSum () const

Compute the sum of all values by reading the data.

- float getUndecidable (const ibis::qDiscreteRange \&cmp, ibis::bitvector \&iffy) const

- float getUndecidable (const ibis::qContinuousRange \&cmp, ibis::bitvector \&iffy) const

Compute the locations of the rows can not be decided by the index.

- void indexSpec (const char $*$ spec)

- const char $*$ indexSpec () const

- void indexSpeedTest () const

- bool isInteger () const 
- bool isNumeric () const

- virtual void loadIndex (const char $*$ opt=0) const throw ()

- void logMessage (const char $*$ event, const char $* \mathrm{fmt}, \ldots$ ) const

- void logWarning (const char $*$ event, const char $* \mathrm{fmt}, \ldots$ ) const

- void lowerBound (double d)

- const double \& lowerBound () const

- const char $*$ name () const

- char $*$ nullMaskName () const

Name of the NULL mask file.

- uint32_t numBins () const

- void preferredBounds (std::vector $<$ double $>\&$ ) const

- virtual void print (std::ostream \&out) const

- void purgeIndexFile (const char $*$ dir $=0$ ) const

- array_t $<$ char $>*$ selectBytes (const bitvector \&mask) const

Return selected rows of the column as an array_t object.

- array_t $\mathrm{t}<$ double $>*$ selectDoubles (const bitvector \&mask) const

Put the selected values into an array as doubles.

- array_t $\mathrm{t}$ float $>*$ selectFloats (const bitvector \&mask) const

Put selected values of a float column into an array.

- array_t $<$ int32_t $>*$ selectInts (const bitvector \&mask) const

- array_t $<$ int64_t $>*$ selectLongs (const bitvector \&mask) const

Can be called on all integral types.

- array_t $<$ int16_t $>*$ selectShorts (const bitvector \&mask) const

Can convert all integers 2-byte or less in length.

- virtual std::vector $<$ std::string $>*$ selectStrings (const bitvector \&mask) const

- virtual array_t $<$ uint32_t $>*$ selectUInts (const bitvector \&mask) const

Can be called on columns of unsigned integral types, UINT, CATEGORY, USHORT, and UBYTE.

- array_t $<$ uint64_t $>*$ selectULongs (const bitvector \&mask) const

Can be called on all unsigned integral types.

- template $<$ typename $\mathrm{T}>$ long selectValues (const bitvector \&mask, array_t $<\mathrm{T}>$ \&vals, array_t $<$ uint32_t $>$ \&inds) const

Select the values marked in the bitvector mask.

- long truncateData (const char $*$ dir, uint32_t nent, ibis::bitvector \&mask) const

truncate the number of data entries in the named dir to nent.

- ibis::TYPE_T type () const

Note:

Name and type can not be changed.

- void unloadIndex () const

- void upperBound (double d)

- const double \& upperBound () const

- virtual void write (FILE $*$ file) const

Write the current content to the TDC file. 
- virtual long writeData (const char $*$ dir, uint32_t nold, uint32_t nnew, ibis::bitvector \&mask, const void $*$ va1, const void $* \mathrm{va} 2=0$ )

Record the content in array val to directory dir. Extend the mask.

\section{Protected Member Functions}

- template $<$ typename $\mathrm{T}>$ void actualMinMax (const array_t $<\mathrm{T}>$ \& vals, const ibis::bitvector \&mask, double \&min, double \&max) const

- void actualMinMax (const char $*$ fname, const ibis::bitvector \&mask, double \&min, double \&max) const

Compute the actual minimum and maximum values.

- template $<$ typename $\mathrm{T}>\mathrm{T}$ computeMax (const array_t $<\mathrm{T}>$ \&vals, const ibis::bitvector \&mask) const

- double computeMax () const

- template $<$ typename $\mathrm{T}>\mathrm{T}$ computeMin (const array_t $<\mathrm{T}>$ \&vals, const ibis::bitvector \&mask) const

- double computeMin () const

- template $<$ typename $\mathrm{T}>$ double computeSum (const array_t $<\mathrm{T}>$ \&vals, const ibis::bitvector \&mask) const

- double computeSum () const

- void logError (const char $*$ event, const char $* \mathrm{fmt}, \ldots$ ) const

- long string2int (int fptr, dictionary \&dic, uint32_t nbuf, char $*$ buf, array_t $<$ uint32_t $>$ \&out) const

\section{Protected Attributes}

- ibis::index $*$ idx

- double lower

- std::string m_bins

- std::string m_desc

- std::string m_name

- ibis::TYPE_T m_type

- ibis::bitvector mask

- const part $*$ theTable

- double upper

\section{Friends}

- class indexLock

- class mutexLock

- class writeLock

\section{Classes}

- class indexLock

A class for controlling access of the index object of a column.

- class info

Some basic information about a column.

- class mutexLock

Provide a mutual exclusion lock on an ibis::column.

- class writeLock

Provide a write lock on a ibis::column object. 


\subsubsection{Detailed Description}

The class to represent a column of a data table.

IBIS represents user data as tables where each table consists of a number of columns. Internally, the data values for each column is stored separated from others. In relational algebra terms, this is equivalent to projecting out each attribute of a relation separately. It increases the efficiency of searching on relatively small number of attributes compared to the horizontal data organization used in typical relational database systems.

\subsubsection{Constructor \& Destructor Documentation}

\subsubsection{1 ibis::column::column (const part $*$ tbl, FILE $*$ file)}

Reconstitute a column from the content of a file.

\section{Note:}

Assume the calling program has read "Begin Property/Column" already.

A well-formed column must have a valid name, i.e., ! m_name.empty().

\subsubsection{2 ibis::column::column (const column \& rhs)}

copy constructor

Note:

The rwlock can not be copied.

The index is not copied either because reference counting difficulties.

\subsubsection{Member Function Documentation}

3.31.3.1 void ibis::column::actualMinMax (const char $*$ name, const ibis::bitvector \& mask, double \& min, double \& max) const [ protected]

Compute the actual minimum and maximum values.

Given a data file name, read its content to compute the actual minimum and the maximum of the data values. Only deal with four types of values, unsigned int, signed int, float and double.

3.31.3.2 long ibis::column::append (const char $* d t$, const char $* d f$, const uint32_t nold, const uint32_t nnew, const uint32_t nbuf, char $*$ buf) [virtual]

Append new data in directory df to the end of existing data in dt.

- df to end of file in

- dt.

Note:

Since this function does not compute the mininimum and the maximum of the new values, it is important the minimum and the maximum is present in the corresponding table.tdc file. For new data without minimum and maximum, some test functions may fail.

Reimplemented in ibis::text, and ibis::category. 
3.31.3.3 void ibis::column::computeMinMax (const char $*$ dir, double \& min, double \& max) const [virtual]

Compute the actual min/max of the data in directory dir.

Report the actual min/max found back through output arguments min and max.

\subsubsection{4 void ibis::column::computeMinMax () [virtual]}

Compute the actual min/max values by actually going through all the values.

This function reads the data in the active data directory and modifies the member variables to record the actual $\min / \max$.

\subsubsection{5 char $*$ ibis::column::dataFileName (const char $* d i r=0)$ const}

Name of the data file in the given data directory.

If the directory name is not given, the directory is assumed to be the current data directory of the table.

\subsubsection{6 long ibis::column::estimateRange (const ibis::qContinuousRange \& $\mathrm{cmp}$ ) const}

Use an index to compute an upper bound on the number of hits.

If no index can be computed, it will return the number of rows as the upper bound.

3.31.3.7 long ibis::column::estimateRange (const ibis::qContinuousRange \& cmp, ibis::bitvector \& low, ibis: :bitvector \& high) const

Compute a lower bound and an upper bound on the number of hits using the bitmap index.

If no index is available a new one will be built. If no index can be built, the lower bound will contain nothing and the the upper bound will contain everything. The two bounds are returned as bitmaps which marked the qualified rows as one, where the lower bound is stored in 'low' and the upper bound is stored in 'high'. If the bitvector 'high' has less bits than 'low', the bitvector 'low' is assumed to have an exact solution. This function always returns zero (0).

3.31.3.8 long ibis::column::evaluateRange (const ibis::qContinuousRange \& cmp, const ibis::bitvector \& mask, ibis::bitvector \& res) const

Attempt to compute the exact answer.

If successful, return the number of hits, otherwise return a negative value.

\subsubsection{9 virtual const char $*$ ibis: column::findString (const char $*$ str) const [inline, virtual]}

Determine if the input string is one of the records.

If yes, return the pointer to the incoming string, otherwise return nil.

Reimplemented in ibis::text.

\subsubsection{0 double ibis::column::getActualMax () const}

Compute the actual maximum value by reading the data or examining the index.

It returns -DBL_MAX in case of error.

\subsubsection{1 double ibis::column::getActualMin () const}

Compute the actual minimum value by reading the data or examining the index.

It returns DBL_MAX in case of error. 
3.31.3.12 long ibis::column::getCumulativeDistribution (std::vector $<$ double $>\&$ bounds, std::vector $<$ uint32_t $>\&$ counts) const

Compute the actual data distribution.

It will generate an index for the column if one is not already available. The value in cts[i] is the number of values less than bds[i]. If there is no NULL values in the column, the array cts will start with 0 and and end the number of rows in the data. The array bds will end with a value that is greater than the actual maximum value.

3.31.3.13 long ibis::column::getDistribution (std::vector $<$ double $>\&$ bbs, std::vector $<$ uint32_t $>$ \& counts) const

Count the number of records in each bin.

The array bins contains bin boundaries that defines the following bins: (.., bins[0]) [bins[0], bins[1]) ... [bins.back(), ...). Because of the two open bins at the end, $\mathrm{N}$ bin boundaries defines $\mathrm{N}+1$ bins. The array counts has one more element than bins. This function returns the number of bins. If this function was executed successfully, the return value should be the same as the size of array counts, and one larger than the size of array bbs.

\subsubsection{4 array_t $<$ int32_t $>*$ ibis::column::getIntArray () const}

Return all rows of the column as an array_t object.

Caller is responsible for deleting the returned object.

\subsubsection{5 virtual const char* ibis::column::getString (uint32_t $\boldsymbol{i}$ ) const [inline, virtual]}

Return the internal string value for the integer.

Only valid for ibis::text and ibis::category. ibis::category ibis::text

3.31.3.16 float ibis::column::getUndecidable (const ibis::qContinuousRange \& $c m p$, ibis::bitvector \& iffy) const

Compute the locations of the rows can not be decided by the index.

Returns the fraction of rows might satisfy the specified range condition.

\subsubsection{7 array_t $<$ char $>*$ ibis::column::selectBytes (const bitvector $\&$ mask) const}

Return selected rows of the column as an array_t object.

Caller is responsible for deleting the returned object.

\subsubsection{8 array_t $<$ double $>*$ ibis::column::selectDoubles (const bitvector $\&$ mask) const}

Put the selected values into an array as doubles.

Note:

Any column type could be selected as doubles. Other selectXXXs function only work on the same data type. This is the only function that allows one to convert to a different type. This is mainly to

\subsubsection{9 array_t $<$ int64_t $>*$ ibis::column::selectLongs (const bitvector $\&$ mask) const}

Can be called on all integral types.

Note that 64-byte unsigned integers are simply treated as signed integer. This may cause the values to be interperted incorrectly. Shorter version of unsigned integers are treated correctly as positive values. 


\subsubsection{0 array_t $<$ int16_t $>*$ ibis::column::selectShorts (const bitvector $\&$ mask) const}

Can convert all integers 2-byte or less in length.

Note that unsigned integers are simply treated as signed integers. Shoter types of signed integers are treated correctly as positive values.

3.31.3.21 template $<$ typename $\mathbf{T}>$ long ibis::column::selectValues (const bitvector $\&$ mask, array_t $<\mathbf{T}>\boldsymbol{\&}$ vals, array_t $<$ uint32_t $>$ \& inds) const

Select the values marked in the bitvector mask.

Select all values marked 1 in the mask and pack them into the output array vals and fill the array inds with the positions of the values selected. On a successful executation, it returns the number of values selected. If it returns zero (0), the contents of vals and inds are not modified. If it returns a negative number, the contents of arrays vals and inds are not guaranteed to be in particular state.

\subsubsection{2 long ibis::column::truncateData (const char $*$ dir, uint32_t nent, ibis::bitvector \& mask) const} truncate the number of data entries in the named dir to nent.

Adjust the null mask accordingly.

The documentation for this class was generated from the following files:

- column.h

- column.cpp

\subsection{2 ibis::column::indexLock Class Reference}

A class for controlling access of the index object of a column.

\#include <column.h>

\section{Public Member Functions}

- const ibis::index $*$ getIndex () const

- indexLock (const ibis::column $*$ col, const char $* \mathrm{~m}$ )

\subsubsection{Detailed Description}

A class for controlling access of the index object of a column.

It directly accesses two member variables of ibis::column class, idx and idxcnt.

The documentation for this class was generated from the following file:

- column.h

\subsection{3 ibis::column::info Class Reference}

Some basic information about a column.

\#include <column.h>

Public Member Functions

- info (const ibis::column \&col) 


\section{Public Attributes}

- const char $*$ description

A description about the column.

- const double expectedMax

The expected upper bound.

- const double expectedMin

The expected lower bound.

- const char $*$ name

Column name.

- const ibis::TYPE_T type

The type of the values.

\subsubsection{Detailed Description}

Some basic information about a column.

Can only be used if the original column used to generate the info object exists in memory.

The documentation for this class was generated from the following file:

- column.h

\subsection{4 ibis::column::mutexLock Class Reference}

Provide a mutual exclusion lock on an ibis::column.

\#include <column.h>

\section{Public Member Functions}

- mutexLock (const ibis::column $*$ col, const char $* \mathrm{~m}$ )

\subsubsection{Detailed Description}

Provide a mutual exclusion lock on an ibis::column.

The documentation for this class was generated from the following file:

- column.h

\subsection{5 ibis::column::writeLock Class Reference}

Provide a write lock on a ibis::column object.

\#include <column.h>

Public Member Functions

- writeLock (const ibis::column $*$ col, const char $* \mathrm{~m})$ 


\subsubsection{Detailed Description}

Provide a write lock on a ibis::column object.

The documentation for this class was generated from the following file:

- column.h

\subsection{6 ibis::colValues Class Reference}

A pure virtual base class.

\#include <colvalues.h>

Inheritance diagram for ibis::colValues::

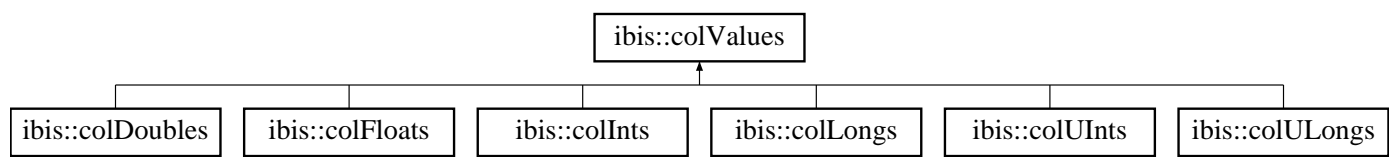

\section{Public Member Functions}

- virtual void bottomk (uint32_t $\mathrm{k}$, array_t $\mathrm{t}<$ uint32_t $>$ \&ind) const $=0$

Return the positions of the $\mathrm{k}$ smallest elements.

- bool canSort () const

- virtual uint32_t elementSize () const $=0$

- virtual bool empty () const=0

- virtual void erase (uint32_t $i$, uint32_t $j$ ) $=0$

- virtual void $*$ getArray () const $=0$

Return the pointer to the pointer to underlying array_t $t<T>$ object.

- virtual double getDouble (uint32_t) const $=0$

- virtual float getFloat (uint32_t) const $=0$

- virtual int32_t getInt (uint32_t $)$ const $=0$

- virtual int64_t getLong (uint32_t) const $=0$

- virtual double getMax () const=0

- virtual double getMin () const $=0$

- virtual double getSum () const=0

- virtual ibis::TYPE_T getType () const=0

Return the type of the data stored.

- virtual uint32_t getUInt (uint32_t) const $=0$

- virtual uint64_t getULong (uint32_t) const $=0$

- virtual const ibis::column $*$ operator $\rightarrow()$ const $=0$

Provide a pointer to the column containing the selected values.

- virtual void reduce (const array_t $<$ uint32_t $>$ \&starts, ibis::selected::FUNCTION func) $=0$

- virtual void reduce (const array_t $<$ uint32_t $>\&$ starts) $=0$

- virtual void reorder (const array_t $<$ uint32_t $>$ \&ind $)=0$

Reorder the values according to the specified indices.

- virtual array_t $<$ uint32_t $>*$ segment $($ const array_t $<$ uint32_t $>*$ old $=0$ ) const $=0$ 
Produce an array of the starting positions of values that are the same.

- virtual uint32_t size () const=0

- virtual void sort (uint32_t i, uint32_t j, array_t $<$ uint32_t $>$ \&neworder) const $=0$

Sort rows in the range $[i, j)$.

- virtual void sort (uint32_t i, uint32_t j, bundle $*$ bdl, colList::iterator head, colList::iterator tail)=0

Sort rows in the range $[i, j)$.

- virtual void sort (uint32_t i, uint32_t j, bundle $*$ bdl) $=0$

Sort rows in the range $[i, j)$.

- void swap (colValues \&rhs)

- virtual void swap (uint32_t i, uint32_t j)=0

- virtual void topk (uint32_t k, array_t $<$ uint32_t $>$ \&ind) const $=0$

Return the positions of the $\mathrm{k}$ largest elements.

- virtual long truncate (uint32_t keep) $=0$

Truncate the number element to no more than keep.

- virtual void write (std::ostream \&out, uint32_t i) const=0

Write ith element as text.

- virtual uint32_t write (FILE $*$ fptr) const=0

Write out whole array as binary.

\section{Static Public Member Functions}

- static colValues $*$ create (const ibis::column $*$ c, void $*$ vals)

Construct from content of an array_t .

- static colValues $*$ create (const ibis::column $* \mathrm{c}$, ibis::fileManager::storage $*$ store, const uint32_t start, const uint32_t nelm)

Construct from content of the file (pointed by store).

- $\quad$ static colValues $*$ create (const ibis::column $*$ c, const ibis::bitvector \&hits)

Construct from a hit vector.

\section{Protected Member Functions}

- colValues (const ibis::column $* \mathrm{c}$ )

\section{Protected Attributes}

- const ibis::column $*$ col

The column where the value is from.

\subsubsection{Detailed Description}

A pure virtual base class. 


\subsubsection{Member Function Documentation}

\subsubsection{1 virtual void ibis::colValues::reorder (const array_t $<$ uint32_t $>$ \& ind) [pure virtual]}

Reorder the values according to the specified indices.

New $[i]=$ Old[ind [i]].

Implemented in ibis::colInts, ibis::colUInts, ibis::colLongs, ibis::colULongs, ibis::colFloats, and ibis::colDoubles.

3.36.2.2 virtual void ibis::colValues::sort (uint32_t $i$, uint32_t $j$, array_t $<$ uint32_t $>$ \& neworder) const [pure virtual]

Sort rows in the range $[i, j)$.

Output the new order in array neworder.

Implemented in ibis::colInts, ibis::colUInts, ibis::colLongs, ibis::colULongs, ibis::colFloats, and ibis::colDoubles.

3.36.2.3 virtual void ibis::colValues::sort (uint32_t $i$, uint32_t $j$, bundle $* b d l$, colList::iterator head, colList::iterator tail) [pure virtual]

Sort rows in the range $[i, j)$.

Also sort the columns between [head, tail).

Implemented in ibis::colInts, ibis::colUInts, ibis::colLongs, ibis::colULongs, ibis::colFloats, and ibis::colDoubles.

The documentation for this class was generated from the following files:

- colValues.h

- colValues.cpp

\subsection{7 ibis::compRange Class Reference}

The class compRange stores computed ranges.

\#include <qExpr.h>

Inheritance diagram for ibis::compRange::

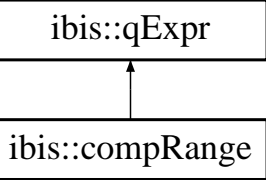

Public Types

- enum OPERADOR \{

UNKNOWN = 0, BITOR, BITAND, PLUS,

MINUS, MULTIPLY, DIVIDE, NEGATE,

POWER \}

- enum STDFUN1 \{

ACOS = 0, ASIN, ATAN, CEIL,

COS, COSH, EXP, FABS,

FLOOR, FREXP, LOG10, LOG, 
MODF, SIN, SINH, SQRT,

TAN, TANH \}

- enUm STDFUN2 $\{$ ATAN2 $=0$, FMOD, LDEXP, POW $\}$

- enUm TERM_TYPE \{

UNDEFINED, VARIABLE, NUMBER, STRING,

OPERATOR, STDFUNCTION1, STDFUNCTION2, CUSTOMFUNCTION1, CUSTOMFUNCTION2 \}

\section{Public Member Functions}

- compRange (const compRange \&rhs)

- compRange (ibis::compRange::term *me1, ibis::qExpr::COMPARE lop, ibis::compRange::term *me2, ibis::qExpr::COMPARE rop, ibis::compRange::term $*$ me3)

- compRange (ibis::compRange::term $*$ me1, COMPARE lop, ibis::compRange::term $*$ me2)

- virtual qExpr $*$ dup () const

Duplicate this object and return a pointer to the new copy.

- const term $*$ getTerm3 () const

- term $*$ getTerm3 ()

- bool inRange () const

Evaluate the logical expression.

- virtual bool isSimple () const

Is the expression simple, i.e., containing only simple range conditions joined with logical operators?

- bool isSimpleRange () const

Is this a simple range expression that can be stored as ibis::qRange?

- ibis::qExpr::COMPARE leftOperator () const

- bool maybeStringCompare () const

Is the string a possible simple string comparison.

- virtual void print (std::ostream \&) const

Print the query expression.

- ibis::qExpr::COMPARE rightOperator () const

- void setTerm3 (term $*$ t)

- ibis::qContinuousRange $*$ simpleRange () const

\section{Static Public Attributes}

- static char $*$ operator_name [ ]

- static char $*$ stdfun1_name []

- static char $*$ stdfun2_name []

\section{Classes}

- class barrel

A barrel to hold a list of variables.

- class bediener 
- class literal

- class number

- class stdFunction1

- class stdFunction2

- class term

- class variable

\subsubsection{Detailed Description}

The class compRange stores computed ranges.

It is for those comparisons involving nontrivial arithmetic expression.

The documentation for this class was generated from the following files:

- qExpr.h

- qExpr.cpp

\subsection{8 ibis::compRange::barrel Class Reference}

A barrel to hold a list of variables.

\#include <qExpr.h>

\section{Public Member Functions}

- barrel (const term $*$ const $\mathrm{t}$ )

- bool equivalent (const barrel \&rhs) const

Is the given barrel of variables equivalent to this one?

- const char $*$ name (uint32_t i) const

- uint32_t recordVariable (const char $*$ name)

Record the specified name.

- void recordVariable (const term $*$ const t)

Record the variable names appear in the term.

- uint32_t size () const

- double \& value (uint32_t i)

- const double \& value (uint32_t i) const

\section{Protected Types}

- typedef std::map $<$ const char $*$, uint32_t, ibis::lessi $>$ termMap

\section{Protected Member Functions}

- double getValue (const char $* \mathrm{~nm}$ ) const

Return the value of the named variable.

- double getValue (uint32_t i) const 


\section{Protected Attributes}

- std::vector $<$ const char $*>$ namelist

List of variable names.

- termMap varmap

Associate a variable name with a position in varvalues and namelist.

- std::vector $<$ double $>$ varvalues

All values are casted to double.

\section{Friends}

- class variable

\subsubsection{Detailed Description}

A barrel to hold a list of variables.

\subsubsection{Member Function Documentation}

\subsubsection{1 uint32_t ibis::compRange::barrel::recordVariable (const char $*$ name) $\quad$ [ in line ]}

Record the specified name.

Return the variable number that is to be used later in functions name and value for retrieving the variable name and its value.

The documentation for this class was generated from the following files:

- qExpr.h

- qExpr.cpp

\subsection{9 ibis::dictionary Class Reference}

Provide a mapping between strings and integers.

\#include <category.h>

\section{Public Types}

- typedef std::map $<$ const char $*$, uint32_t, ibis::lessi $>$ wordList

\section{Public Member Functions}

- void clear ()

- dictionary (const dictionary \&dic)

- const char $*$ find (const char $*$ str) const

Is the given string in the dictionary? Return a null pointer if not.

- uint32_t insert (const char $*$ str)

Insert a string if not in dictionary. 
- uint32_t insertRaw (char $*$ str)

Insert a string if not in dictionary.

- uint32_t operator[] (const char $*$ str) const

Return an integer corresponding to the string.

- const char $*$ operator[ ] (uint32_t i) const

Return a string corresponding to the integer.

- $\operatorname{void}$ read (const char $*$ name)

- uint32_t size () const

Return the number of valid (not null) strings in the dictionary.

- void write (const char $*$ name) const

\section{Protected Member Functions}

- void copy (const dictionary \&rhs)

\subsubsection{Detailed Description}

Provide a mapping between strings and integers.

A utility class used by ibis::category. The NULL string is always the 0th string.

\subsubsection{Member Function Documentation}

\subsubsection{1 const char $*$ ibis::dictionary::find (const char $*$ str) const $\quad$ [ inline ]}

Is the given string in the dictionary? Return a null pointer if not.

Otherwise it returns null pointer. This function makes a little easier to determine whether a string is in a dictionary.

\subsubsection{2 uint32_t ibis::dictionary::insertRaw (char $*$ str) $\quad$ [ inline ]}

Insert a string if not in dictionary.

Do not make a copy of the input string. Caller needs to check whether it is a new word in the dictionary. If it is not a new word in the dictionary, the dictionary does not take ownership of the string argument.

The documentation for this class was generated from the following files:

- category.h

- category.cpp

\subsection{0 ibis::direkte Class Reference}

Directly use the integer values as bin number to avoid some intemdiate steps.

\#include <idirekte.h>

Inheritance diagram for ibis::direkte:: 


\section{ibis::index \\ ibis::direkte}

\section{Public Member Functions}

- virtual long append (const char $* \mathrm{dt}$, const char $* \mathrm{df}$, uint32_t nnew)

Extend the index.

- virtual void binBoundaries (std::vector $<$ double $>\&$ ) const

The function binBoundaries and binWeights return bin boundaries and counts of each bin respectively.

- virtual void binWeights (std::vector $<$ uint32_t $>\&$ ) const

- direkte (const ibis::column $* \mathrm{c}$, ibis::fileManager::storage $*$ st, uint32_t offset=8)

- direkte (const ibis::column $* \mathrm{c}$, const char $* \mathrm{f}=0$ )

Constructing a new ibis::direkte object from base data in a file.

- virtual uint32_t estimate (const ibis::qDiscreteRange \&expr) const

- virtual void estimate (const ibis::qDiscreteRange \&expr, ibis::bitvector \&lower, ibis::bitvector \&upper) const

Estimate the hits for discrete ranges, i.e., those translated from 'a IN (x, y, .

- virtual uint32_t estimate (const ibis::qContinuousRange \&expr) const

Returns an upper bound on the number of hits.

- virtual void estimate (const ibis::qContinuousRange \&expr, ibis::bitvector \&lower, ibis::bitvector \&upper) const

Computes an approximation of hits as a pair of lower and upper bounds.

- virtual double estimateCost (const ibis::qDiscreteRange \&expr) const

- virtual double estimateCost (const ibis::qContinuousRange \&expr) const

Estimate the code of evaluate a range condition.

- virtual long evaluate (const ibis::qDiscreteRange \&expr, ibis::bitvector \&hits) const

- virtual long evaluate (const ibis::qContinuousRange \&expr, ibis::bitvector \&hits) const

To evaluate the exact hits.

- virtual long getCumulativeDistribution (std::vector $<$ double $>$ \&bds, std::vector $<$ uint32_t $>$ \&cts) const

Cumulative distribution of the data.

- virtual long getDistribution (std::vector $<$ double $>\& b b s$, std::vector $<$ uint32_t $>\&$ cts) const

Binned distribution of the data.

- virtual double getMax () const

The maximum value recorded in the index.

- virtual double getMin () const

The minimum value recorded in the index.

- virtual double getSum () const

Compute the approximate sum of all the values indexed. 
- virtual const char $*$ name () const

Returns the name of the index, similar to the function type, but returns a string instead.

- virtual void print (std::ostream \&out) const

Prints human readable information.

- virtual void read (ibis::fileManager::storage $*$ st)

Reconstructs an index from an array of bytes.

- virtual void read (const char $*$ name)

Reconstructs an index from the named file.

- virtual void speedTest (std::ostream \&out) const

Time some logical operations and print out their speed.

- virtual INDEX_TYPE type () const

Returns an index type identifier.

- virtual float undecidable (const ibis::qDiscreteRange \&expr, ibis::bitvector \&iffy) const

- virtual float undecidable (const ibis::qContinuousRange \&expr, ibis::bitvector \&iffy) const

Mark the position of the rows that can not be decided with this index.

- virtual void write (const char $*$ name) const

Write the direct bitmap index to a file.

\section{Protected Member Functions}

- template $<$ typename $\mathrm{T}>$ int construct $($ const char $* \mathrm{f}$ )

- direkte (const direkte \&)

- void locate (const ibis::qContinuousRange \&expr, uint32_t \&hit0, uint32_t \&hit1) const

- const direkte $\&$ operator $=($ const direkte $\&)$

\subsubsection{Detailed Description}

Directly use the integer values as bin number to avoid some intemdiate steps.

\subsubsection{Member Function Documentation}

3.40.2.1 void ibis::direkte::estimate (const ibis::qDiscreteRange \& expr, ibis::bitvector \& lower, ibis::bitvector \& upper) const [virtual]

Estimate the hits for discrete ranges, i.e., those translated from 'a IN (x, y, .

.)'.

Reimplemented from ibis::index. 
3.40.2.2 void ibis::direkte::estimate (const ibis::qContinuousRange \& expr, ibis::bitvector \& lower, ibis::bitvector \& upper) const [virtual]

Computes an approximation of hits as a pair of lower and upper bounds.

\section{Parameters:}

expr the query expression to be evaluated.

lower a bitvector marking a subset of the hits. All rows marked with one (1) are definitely hits.

upper a bitvector marking a superset of the hits. All hits are marked with one, but some of the rows marked one may not be hits. If the variable upper is empty, the variable lower is assumed to contain the exact answer.

Implements ibis::index.

3.40.2.3 long ibis::direkte::evaluate (const ibis::qContinuousRange \& expr, ibis::bitvector \& hits) const [virtual]

To evaluate the exact hits.

On success, return the number of hits, otherwise a negative value is returned.

Implements ibis::index.

\subsubsection{4 double ibis::direkte::getSum () const [virtual]}

Compute the approximate sum of all the values indexed.

If it decides that computing the sum directly from the vertical partition is more efficient, it will return NaN immediately. Implements ibis::index.

\subsubsection{5 void ibis::direkte::print (std::ostream \& out) const [virtual]}

Prints human readable information.

Outputs information about the index as text to the specified output stream.

Implements ibis::index.

\subsubsection{6 void ibis::direkte::read (ibis::fileManager::storage $* s t$ ) [virtua l]}

Reconstructs an index from an array of bytes.

Intended for internal use only!

Implements ibis::index.

\subsubsection{7 void ibis::direkte::read (const char $*$ name) $\quad$ [virtual]}

Reconstructs an index from the named file.

The name can be the directory containing an index file. In this case, the name of the index file must be the name of the column followed by ".idx" suffix.

Implements ibis::index.

3.40.2.8 virtual float ibis::direkte::undecidable (const ibis::qContinuousRange \& expr, ibis::bitvector \& iffy) const [inline, virtual]

Mark the position of the rows that can not be decided with this index. 


\section{Parameters:}

expr the range conditions to be evaluated.

iffy the bitvector marking the positions of rows that can not be decided using the index. Return value is the expected fraction of undecided rows that might satisfy the range conditions.

Implements ibis::index.

The documentation for this class was generated from the following files:

- idirekte.h

- idirekte.cpp

\subsection{1 ibis::discretePoisson Class Reference}

Discrete random number with Poisson distribution exp(-x/lambda).

\#include <twister.h>

\section{Public Member Functions}

- discretePoisson (ibis::uniformRandomNumber $*$ ur, const double lam=1.0, long $\mathrm{m}=0$ )

- long next ()

- long operator() ()

\subsubsection{Detailed Description}

Discrete random number with Poisson distribution exp(-x/lambda).

Use the rejection-inversion algorithm of W. Hormann and G. Derflinger.

The documentation for this class was generated from the following file:

- twister.h

\subsection{2 ibis::discretePoisson1 Class Reference}

Specialized version of the Poisson distribution $\exp (-\mathrm{x})$.

\#include <twister.h>

\section{Public Member Functions}

- discretePoisson1 (ibis::uniformRandomNumber $*$ ur)

- long next ()

- long operator() ()

\subsubsection{Detailed Description}

Specialized version of the Poisson distribution $\exp (-\mathrm{x})$.

The documentation for this class was generated from the following file:

- twister.h 


\subsection{3 ibis::discreteZipf Class Reference}

Discrete Zipf distribution: $\mathrm{p}(\mathrm{k})$ is proportional to $(\mathrm{v}+\mathrm{k})^{\wedge}(-\mathrm{a})$ where $\mathrm{a}>1, \mathrm{k}>=0$.

\#include <twister.h>

\section{Public Member Functions}

- discreteZipf (ibis::uniformRandomNumber $*$ ur, double a=2.0, unsigned long $v=1$, unsigned long imax=ULONG_MAX)

- unsigned long next ()

- unsigned long operator() ()

\subsubsection{Detailed Description}

Discrete Zipf distribution: $\mathrm{p}(\mathrm{k})$ is proportional to $(\mathrm{v}+\mathrm{k})^{\wedge}(-\mathrm{a})$ where $\mathrm{a}>1, \mathrm{k}>=0$.

It uses the rejection-inversion algorithm of W. Hormann and G. Derflinger. The values generated are in the range of $[0$, imax $]$ (inclusive, both ends are included).

The documentation for this class was generated from the following file:

- twister.h

\subsection{4 ibis::discreteZipf1 Class Reference}

A specialized case of the Zipf distribution $f(x)=1 /(1+x)$.

\#include <twister.h>

\section{Public Member Functions}

- discreteZipf1 (ibis::uniformRandomNumber $*$ ur, unsigned long imax=100)

- unsigned long next ()

- unsigned long operator() ()

\subsubsection{Detailed Description}

A specialized case of the Zipf distribution $f(x)=1 /(1+x)$.

The general case discrateZipf requires a $>1$.

The documentation for this class was generated from the following file:

- twister.h

\subsection{5 ibis::discreteZipf2 Class Reference}

A specialized version of the Zipf distribution $\mathrm{f}(\mathrm{x})=1 /(1+\mathrm{x})^{\wedge} 2$.

\#include <twister.h> 


\section{Public Member Functions}

- discreteZipf2 (ibis::uniformRandomNumber $*$ ur, unsigned long imax=ULONG_MAX)

- unsigned long next ()

- unsigned long operator() ()

Return a discrete random number in the range of [0, imax].

\subsubsection{Detailed Description}

A specialized version of the Zipf distribution $f(x)=1 /(1+x)^{\wedge} 2$.

Should be much faster than using discreteZipf $(2,1$, imax $)$.

The documentation for this class was generated from the following file:

- twister.h

\subsection{6 ibis::egale Class Reference}

The multicomponent equality code on bins.

\#include <ibin.h>

Inheritance diagram for ibis::egale::

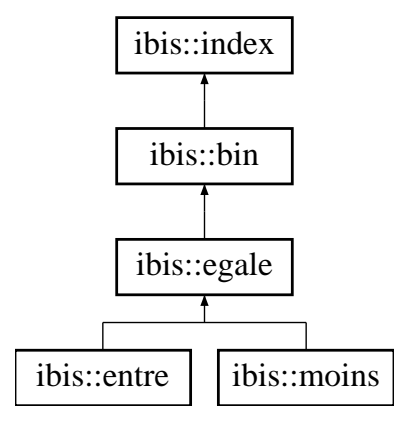

\section{Public Member Functions}

- long append (const array_t $\mathrm{t}<$ uint32_t $>$ \&ind)

Append a list of integers representing bin numbers.

- long append (const ibis::egale \&tail)

- virtual long append (const char $* \mathrm{dt}$, const char $* \mathrm{df}$, uint32_t nnew)

Extend the index.

- virtual void binBoundaries (std::vector $<$ double $>\&$ b) const

The function binBoundaries and binWeights return bin boundaries and counts of each bin respectively.

- virtual void binWeights (std::vector $<$ uint32_t $>\& b$ ) const

- egale (const ibis::bin \&rhs, const uint32_t nbase=2)

- egale (const ibis::column $*$ c, ibis::fileManager::storage $*$ st, uint32_t offset=8)

- egale (const ibis::column $* \mathrm{c}=0$, const char $* \mathrm{f}=0$, const uint32_t nbase $=2$ )

- virtual uint32_t estimate (const ibis::qContinuousRange \&expr) const

Returns an upper bound on the number of hits. 
- virtual void estimate (const ibis::qContinuousRange \&expr, ibis::bitvector \&lower, ibis::bitvector \&upper) const

Computes an approximation of hits as a pair of lower and upper bounds.

- virtual long evaluate (const ibis::qContinuousRange \&expr, ibis::bitvector \&hits) const

To evaluate the exact hits.

- virtual double getSum () const

Compute the approximate sum of all the values indexed.

- virtual const char $*$ name () const

Returns the name of the index, similar to the function type, but returns a string instead.

- virtual void print (std::ostream \&out) const

Prints human readable information.

- virtual void read (ibis::fileManager::storage $*$ st)

Reconstructs an index from an array of bytes.

- virtual void read (const char $*$ idxfile)

Reconstructs an index from the named file.

- virtual void speedTest (std::ostream \&out) const

Time some logical operations and print out their speed.

- virtual INDEX_TYPE type () const

Returns an index type identifier.

- virtual float undecidable (const ibis::qContinuousRange \&expr, ibis::bitvector \&iffy) const

Mark the position of the rows that can not be decided with this index.

- virtual void write (const char $* \mathrm{dt})$ const

Save index to a file.

\section{Protected Member Functions}

- void addBins_(uint32_t ib, uint32_t ie, ibis::bitvector \&res) const

- virtual void clear ()

Clear the existing content.

- virtual double computeSum () const

- void construct (const char $* \mathrm{f}$ )

- egale (const ibis::column $* \mathrm{c}$, const char $* \mathrm{f}$, const array_t $<$ double $>$ \&bd, const array_t $<$ uint32_t $>$ bs)

- void write (int fdes) const

\section{Protected Attributes}

- array_t $<$ uint32_t $>$ bases

- $\operatorname{array} \_t<$ uint $32 \_t>$ cnts

- uint32_t nbases

- uint32_t nbits 


\subsubsection{Detailed Description}

The multicomponent equality code on bins.

The word egale is a French word for 'equal'.

\subsubsection{Member Function Documentation}

3.46.2.1 void ibis::egale::estimate (const ibis::qContinuousRange \& expr, ibis::bitvector \& lower, ibis::bitvector \& upper) const [virtual]

Computes an approximation of hits as a pair of lower and upper bounds.

\section{Parameters:}

expr the query expression to be evaluated.

lower a bitvector marking a subset of the hits. All rows marked with one (1) are definitely hits.

upper a bitvector marking a superset of the hits. All hits are marked with one, but some of the rows marked one may not be hits. If the variable upper is empty, the variable lower is assumed to contain the exact answer.

Reimplemented from ibis::bin.

Reimplemented in ibis::moins, and ibis::entre.

3.46.2.2 long ibis::egale::evaluate (const ibis::qContinuousRange \& expr, ibis::bitvector \& hits) const [virtual]

To evaluate the exact hits.

On success, return the number of hits, otherwise a negative value is returned.

Reimplemented from ibis::bin.

Reimplemented in ibis::moins, and ibis::entre.

\subsubsection{3 double ibis::egale::getSum () const [virtual]}

Compute the approximate sum of all the values indexed.

If it decides that computing the sum directly from the vertical partition is more efficient, it will return NaN immediately.

Reimplemented from ibis::bin.

Reimplemented in ibis::moins, and ibis::entre.

\subsubsection{4 void ibis::egale::print (std::ostream \& out) const [ virtual]}

Prints human readable information.

Outputs information about the index as text to the specified output stream.

Reimplemented from ibis::bin.

Reimplemented in ibis::moins, and ibis::entre.

3.46.2.5 void ibis::egale::read (ibis::fileManager::storage $* s t$ ) [virtual]

Reconstructs an index from an array of bytes.

Intended for internal use only!

Reimplemented from ibis::bin. 


\subsubsection{6 void ibis::egale::read (const char $*$ idxfile) [virtual]}

Reconstructs an index from the named file.

The name can be the directory containing an index file. In this case, the name of the index file must be the name of the column followed by ".idx" suffix.

Reimplemented from ibis::bin.

3.46.2.7 float ibis::egale::undecidable (const ibis::qContinuousRange \& expr, ibis::bitvector \& iffy) const [virtual]

Mark the position of the rows that can not be decided with this index.

\section{Parameters:}

expr the range conditions to be evaluated.

iffy the bitvector marking the positions of rows that can not be decided using the index. Return value is the expected fraction of undecided rows that might satisfy the range conditions.

Reimplemented from ibis::bin.

\subsubsection{8 void ibis::egale::write (const char $* \boldsymbol{d t}$ ) const [virtual]}

Save index to a file.

Outputs the index in a compact binary format to the named file or directory. The index file contains a header that can be identified by the function isIndex.

Reimplemented from ibis::bin.

Reimplemented in ibis::moins, and ibis::entre.

The documentation for this class was generated from the following files:

- ibin.h

- icegale.cpp

\subsection{7 ibis::entre Class Reference}

The multicomponent interval code on bins.

\#include <ibin.h>

Inheritance diagram for ibis::entre::

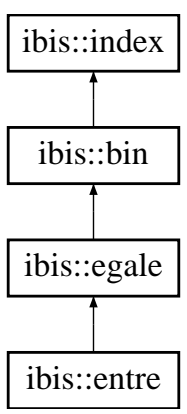

\section{Public Member Functions}

- long append (const array_t $<$ uint32_t $>$ \&ind) 
Append a list of integers representing bin numbers.

- long append (const ibis::entre \&tail)

- virtual long append (const char $* \mathrm{dt}$, const char $* \mathrm{df}$, uint32_t nnew)

Extend the index.

- entre (const ibis::bin \&rhs, const uint32_t nbase=2)

- entre (const ibis::column $*$ c, ibis::fileManager::storage $*$ st, uint32_t offset=8)

- entre (const ibis::column $* \mathrm{c}=0$, const char $* \mathrm{f}=0$, const uint32_t nbase $=2$ )

- virtual uint32_t estimate (const ibis::qContinuousRange \&expr) const

Returns an upper bound on the number of hits.

- virtual void estimate (const ibis::qContinuousRange \&expr, ibis::bitvector \&lower, ibis::bitvector \&upper) const

Computes an approximation of hits as a pair of lower and upper bounds.

- virtual long evaluate (const ibis::qContinuousRange \&expr, ibis::bitvector \&hits) const

To evaluate the exact hits.

- virtual double getSum () const

Compute the approximate sum of all the values indexed.

- virtual const char $*$ name () const

Returns the name of the index, similar to the function type, but returns a string instead.

- virtual void print (std::ostream \&out) const

Prints human readable information.

- virtual void speedTest (std::ostream \&out) const

Time some logical operations and print out their speed.

- virtual INDEX_TYPE type () const

Returns an index type identifier.

- virtual void write (const char $* \mathrm{dt}$ ) const

Save index to a file.

\section{Protected Member Functions}

- virtual double computeSum () const

\subsubsection{Detailed Description}

The multicomponent interval code on bins.

Entre is a French word for 'in between'. 


\subsubsection{Member Function Documentation}

3.47.2.1 void ibis::entre::estimate (const ibis::qContinuousRange \& expr, ibis::bitvector \& lower, ibis::bitvector \& upper) const [virtual]

Computes an approximation of hits as a pair of lower and upper bounds.

\section{Parameters:}

expr the query expression to be evaluated.

lower a bitvector marking a subset of the hits. All rows marked with one (1) are definitely hits.

upper a bitvector marking a superset of the hits. All hits are marked with one, but some of the rows marked one may not be hits. If the variable upper is empty, the variable lower is assumed to contain the exact answer.

Reimplemented from ibis::egale.

3.47.2.2 long ibis::entre::evaluate (const ibis::qContinuousRange \& expr, ibis::bitvector \& hits) const [virtual]

To evaluate the exact hits.

On success, return the number of hits, otherwise a negative value is returned.

Reimplemented from ibis::egale.

\subsubsection{3 double ibis::entre::getSum () const [virtual]}

Compute the approximate sum of all the values indexed.

If it decides that computing the sum directly from the vertical partition is more efficient, it will return NaN immediately. Reimplemented from ibis::egale.

\subsubsection{4 void ibis::entre::print (std::ostream \& out) const [virtual]}

Prints human readable information.

Outputs information about the index as text to the specified output stream.

Reimplemented from ibis::egale.

\subsubsection{5 void ibis::entre::write (const char $* \boldsymbol{d t}$ ) const [virtual]}

Save index to a file.

Outputs the index in a compact binary format to the named file or directory. The index file contains a header that can be identified by the function isIndex.

Reimplemented from ibis::egale.

The documentation for this class was generated from the following files:

- ibin.h

- icentre.cpp

\subsection{8 ibis::fade Class Reference}

The multicomponent range-encoded index. 
Inheritance diagram for ibis::fade::

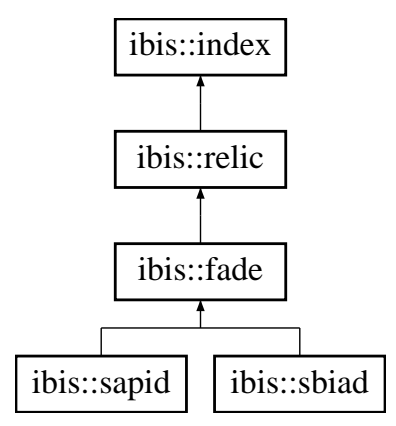

\section{Public Member Functions}

- virtual long append (const char $* \mathrm{dt}$, const char $* \mathrm{df}$, uint32_t nnew)

Extend the index.

- virtual void binWeights (std::vector $<$ uint32_t $>\& b$ ) const

- virtual uint32_t estimate (const ibis::qContinuousRange \&expr) const

Returns an upper bound on the number of hits.

- virtual double estimateCost (const ibis::qContinuousRange \&expr) const

Estimate the code of evaluate a range condition.

- virtual long evaluate (const ibis::qDiscreteRange \&expr, ibis::bitvector \&hits) const

- virtual long evaluate (const ibis::qContinuousRange \&expr, ibis::bitvector \&hits) const

To evaluate the exact hits.

- fade (const ibis::column $*$ c, ibis::fileManager::storage $*$ st, uint32_t offset=8)

- fade (const ibis:: column $* \mathrm{c}=0$, const char $* \mathrm{f}=0$, const uint32_t nbase $=2$ )

- virtual double getSum () const

Compute the approximate sum of all the values indexed.

- virtual const char $*$ name () const

Returns the name of the index, similar to the function type, but returns a string instead.

- virtual void print (std::ostream \&out) const

Prints human readable information.

- virtual void read (ibis::fileManager::storage $*$ st)

Reconstruct an index from a piece of consecutive memory.

- virtual void read (const char $*$ idxfile)

Read the index contained in the file named $\mathrm{f}$.

- virtual void speedTest (std::ostream \&out) const

Time some logical operations and print out their speed.

- virtual INDEX_TYPE type () const

Returns an index type identifier.

- virtual void write (const char $* \mathrm{dt}$ ) const

Save index to a file. 


\section{Protected Member Functions}

- virtual void clear ()

Clear the existing content.

- void write (int fdes) const

\section{Protected Attributes}

- array_t $<$ uint32_t $>$ bases

- $\operatorname{array\_ t}<$ uint32_t $>$ cnts

\subsubsection{Detailed Description}

The multicomponent range-encoded index.

Defined by Chan and Ioannidis (SIGMOD 98).

\subsubsection{Member Function Documentation}

3.48.2.1 long ibis::fade::evaluate (const ibis::qContinuousRange \& expr, ibis::bitvector \& hits) const [virtual]

To evaluate the exact hits.

On success, return the number of hits, otherwise a negative value is returned.

Reimplemented from ibis::relic.

Reimplemented in ibis::sbiad, and ibis::sapid.

\subsubsection{2 double ibis::fade::getSum () const [virtual]}

Compute the approximate sum of all the values indexed.

If it decides that computing the sum directly from the vertical partition is more efficient, it will return NaN immediately. Reimplemented from ibis::relic.

\subsubsection{3 void ibis::fade::print (std::ostream \& out) const [virtual ]}

Prints human readable information.

Outputs information about the index as text to the specified output stream.

Reimplemented from ibis::relic.

Reimplemented in ibis::sbiad, and ibis::sapid.

\subsubsection{4 void ibis::fade::write (const char $* \boldsymbol{d t}$ ) const [virtual]}

Save index to a file.

Outputs the index in a compact binary format to the named file or directory. The index file contains a header that can be identified by the function isIndex.

Reimplemented from ibis::relic.

Reimplemented in ibis::sbiad, and ibis::sapid.

The documentation for this class was generated from the following files: 
- irelic.h

- ifade.cpp

\subsection{9 ibis::fileManager Class Reference}

This fileManager is intended to allow different objects to share the same open file.

\#include <filemanager.h>

\section{Public Types}

- enum ACCESS_PREFERENCE \{ MMAP_LARGE_FILES, PREFER_READ, PREFER_MMAP $\}$ Hint passed to the function get $\mathrm{File.}$

\section{Public Member Functions}

- void addCleaner (const cleaner $* \mathrm{cl}$ )

- void clear ()

Close all files and remove all records of them.

- void flushDir (const char *name)

Close all files in the named directory, but not subdirectories.

- void flushFile (const char $*$ name)

Close the file, remove the record about it from the file manager.

- void gainReadAccess (const char $*$ mesg) const

Obtain a read lock on the file manager.

- int getFile (const char $*$ name, storage $* *$ st, ACCESS_PREFERENCE pref=MMAP_LARGE_FILES)

- template $<$ typename $\mathrm{T}>$ int getFile (const char $*$ name, array_t $<\mathrm{T}>$ \&arr, ACCESS_PREFERENCE pref=MMAP_LARGE_FILES)

- int getFile (const char $*$ name, array_t $t<$ rid_t $>$ \&arr)

- int getFile (const char $*$ name, array_ $\mathrm{t}<$ double $>$ \&arr)

- int getFile (const char $*$ name, array_ $\mathrm{t}<$ float $>$ \&arr)

- int getFile (const char $*$ name, array_t $<$ uint64_t $>$ \&arr)

- int getFile (const char $*$ name, array_t $<$ int64_t $>$ \&arr)

- int getFile (const char $*$ name, array_t $<$ uint32_t $>$ \&arr)

- int getFile (const char $*$ name, array_t $<$ int32_t $>\&$ arr)

- int getFile (const char $*$ name, array_t $<$ unsigned char $>$ \&arr)

- int getFile (const char $*$ name, array_t $<$ char $>$ \&arr)

Given a file name, place the content in an array_t $<T>$.

- storage $*$ getFileSegment (const char $*$ name, off_t b, off_t e)

Read or memory map a portion of the named file (name).

- time_t iBeat () const

Returns the value of simple counter.

- const double \& pageCount () const

Returns the number of pages accessed by function read from stdlib.h. 
- void printStatus (std::ostream \&out) const

Prints status information about the file manager.

- void recordPages (off_t start, off_t stop)

Given the starting and ending addresses, this function computes the number of pages involved.

- void releaseAccess (const char $*$ mesg) const

Release a read lock on the file manager.

- void removeCleaner (const cleaner $* \mathrm{cl})$

- void signalMemoryAvailable () const

Signal to the file manager that some memory have been freed.

- int tryGetFile (const char $*$ name, storage $* *$ st, ACCESS_PREFERENCE pref=MMAP_LARGE_FILES)

Returns 1 if there is not enough space to read the whole file into memory.

- template $<$ typename $\mathrm{T}>$ int tryGetFile (const char $*$ name, array_t $<\mathrm{T}>$ \&arr, ACCESS_PREFERENCE pref=MMAP_LARGE_FILES)

\section{Static Public Member Functions}

- static long unsigned bytesFree ()

Return the number of bytes free.

- static long unsigned bytesInUse ()

Returns the number of bytes currently on records in the file manager.

- static void decreaseUse (size_t dec, const char $*$ evt)

- static void increaseUse (size_t inc, const char $*$ evt)

- static fileManager \& instance ()

Returns a pointer to the one and only file manager.

- static uint32_t pageSize ()

Returns the page size (in bytes) used by the file system.

\section{Protected Member Functions}

- fileManager (const fileManager \&rhs)

- const fileManager \& operator= (const fileManager \&rhs)

- void recordFile (roFile $*$ )

\section{Static Protected Attributes}

- static unsigned long maxBytes

Maximum number of bytes allowed.

- static unsigned int maxOpenFiles

Maximum number of files opened.

- static volatile unsigned long totalBytes

Total bytes of all managed objects. 


\section{Friends}

- class mutexLock

- class roFile

- class storage

- class writeLock

\section{Classes}

- class cleaner

A function object to be used to register external cleaners.

- class mutexLock

Used to prevent simultaneous modification of the two internal lists.

- class readLock

An object who uses a file under the management of the file manager should hold a readLock.

- class roFile

This class manages content of a whole (read-only) file.

- class storage

The storage class treats all memory as char*.

- class writeLock

A write lock for controlling access to the two interval lists.

\subsubsection{Detailed Description}

This fileManager is intended to allow different objects to share the same open file.

It does not manage writing of files.

\subsubsection{Member Enumeration Documentation}

\subsubsection{1 enum ibis::fileManager::ACCESS_PREFERENCE}

Hint passed to the function getFile.

The main choice is whether to use memory map or use the read function to access the content of a file.

\subsubsection{Member Function Documentation}

\subsubsection{1 int ibis::fileManager::getFile (const char $*$ name, array_t $<$ char $>$ \& arr)}

Given a file name, place the content in an array_t $<\mathrm{T}>$.

The return value is zero ( 0 ) if the function is successful, otherwise returns a non-zero value.

3.49.3.2 ibis::fileManager::storage $*$ ibis::fileManager::getFileSegment (const char $*$ name, off_t $b$, off_t $e$ ) Read or memory map a portion of the named file (name).

The file is not tracked by tracking the name through a separate variable in the class rofSegment. 


\subsubsection{3 void ibis::fileManager::recordPages (off_t start, off_t stop) [ in line ]}

Given the starting and ending addresses, this function computes the number of pages involved. Used by derived classes to record page accesses.

3.49.3.4 int ibis::fileManager::tryGetFile (const char $*$ name, storage $* *$ st, ACCESS_PREFERENCE pref $=$ MMAP_LARGE_FILES)

Returns 1 if there is not enough space to read the whole file into memory.

Other return values are same as the function get $\mathrm{File.}$

The documentation for this class was generated from the following files:

- fileManager.h

- fileManager.cpp

\subsection{0 ibis::fileManager::cleaner Class Reference}

A function object to be used to register external cleaners.

\#include <filemanager.h>

\section{Public Member Functions}

- virtual void operator() () const=0

\subsubsection{Detailed Description}

A function object to be used to register external cleaners.

The documentation for this class was generated from the following file:

- fileManager.h

\subsection{1 ibis::fileManager::readLock Class Reference}

An object who uses a file under the management of the file manager should hold a readLock.

\#include <fileManager.h>

\section{Public Member Functions}

- readLock (const char $* \mathrm{~m})$

\subsubsection{Detailed Description}

An object who uses a file under the management of the file manager should hold a readLock.

The documentation for this class was generated from the following file:

- fileManager.h 


\subsection{2 ibis::fileManager::roFile Class Reference}

This class manages content of a whole (read-only) file.

\#include <filemanager.h>

Inheritance diagram for ibis::fileManager::roFile::

$$
\begin{array}{|l|}
\hline \text { ibis::fileManager::storage } \\
\hline \text { ibis::fileManager::roFile } \\
\hline
\end{array}
$$

\section{Public Member Functions}

- virtual void beginUse ()

- virtual void endUse ()

- virtual bool isFileMap () const

Is the storage a file map?

- virtual size_t printStatus (std::ostream \&out) const

- $\operatorname{void}$ read (const char $*$ file)

- float score () const

The function to give score to a file.

\section{Protected Member Functions}

- virtual void clear ()

- void doRead (const char $*$ file, off_t b, off_t e)

- void doRead (const char $*$ file)

- size_t printBody (std::ostream \&out) const

\section{Friends}

- class ibis::fileManager

\subsubsection{Detailed Description}

This class manages content of a whole (read-only) file.

It inherits the basic information stored in fileManager::storage and is intended to process read-only files.

\subsubsection{Member Function Documentation}

\subsubsection{1 float ibis::fileManager::roFile::score () const [inline]}

The function to give score to a file.

Files with smallest scores are the target for removal.

The documentation for this class was generated from the following files:

- fileManager.h

- fileManager.cpp 


\subsection{3 ibis::fileManager::storage Class Reference}

The storage class treats all memory as char*.

\#include <filemanager.h>

Inheritance diagram for ibis::fileManager::storage::

\begin{tabular}{|l|}
\hline ibis::fileManager::storage \\
\hline ibis::fileManager::roFile \\
\hline
\end{tabular}

\section{Public Member Functions}

- const char $*$ begin () const

- char $*$ begin ()

Starting address of the storage object.

- virtual void beginUse ()

- size_t bytes () const

Return the number of bytes contained in the object.

- void copy (const storage \&rhs)

- bool empty () const

Is the storage object empty?

- const char $*$ end () const

- virtual void endUse ()

- void enlarge (size_t nelm=0)

Enlarge the current array by $61.8 \%$ if nelm is smaller than the current size, otherwise enlarge to the specified size.

- const char $*$ filename () const

- unsigned inUse () const

- virtual bool isFileMap () const

Is the storage a file map?

- const storage \& operator= (const storage \&rhs)

- unsigned pastUse () const

- virtual size_t printStatus (std::ostream \&out) const

- off_t read (const int fdes, const off_t begin, const off_t end)

Read part of an open file. Return the number of bytes read.

- size_t size () const

Return the size (bytes) of the object.

- storage (const char $*$ begin, const char $*$ end)

Make another copy of the content in the range [begin, end).

- storage (const storage \&rhs)

- storage (const int fdes, const off_t begin, const off_t end)

Read part of a file [start, end). 
- storage (size_t $\mathrm{n})$

- void swap (storage \&rhs) throw ()

- int unnamed ()

Those storage not associated with files do not have names.

- void write (const char $*$ file) const

Write the content to the named file.

\section{Protected Member Functions}

- virtual void clear ()

\section{Protected Attributes}

- char $* \mathbf{m} \_$begin

- char $* \mathbf{m} \_$end

- unsigned nacc

- char $*$ name

- unsigned nref

\subsubsection{Detailed Description}

The storage class treats all memory as char*.

It only uses malloc family of functions to manage the memory allocation and deallocation.

\subsubsection{Member Function Documentation}

\subsubsection{1 unsigned ibis::fileManager::storage::inUse () const [in line ]}

\subsubsection{2 unsigned ibis::fileManager::storage::pastUse () const [in line ]}

\subsubsection{3 int ibis::fileManager::storage::unnamed () [inline]}

Those storage not associated with files do not have names.

They are not tracked by the file manager and should be immediately freed after use.

The documentation for this class was generated from the following files:

- fileManager.h

- fileManager.cpp

\subsection{4 ibis::fuzz Class Reference}

The two-level interval-equality code.

\#include <irelic.h>

Inheritance diagram for ibis::fuzz:: 


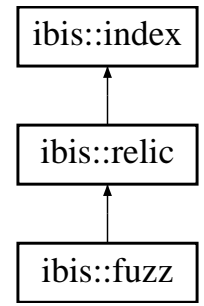

\section{Public Member Functions}

- virtual long append (const char $* \mathrm{dt}$, const char $* \mathrm{df}$, uint32_t nnew)

Extend the index.

- virtual uint32_t estimate (const ibis::qContinuousRange \&expr) const

Returns an upper bound on the number of hits.

- virtual double estimateCost (const ibis::qContinuousRange \&expr) const

Estimate the code of evaluate a range condition.

- virtual long evaluate (const ibis::qContinuousRange \&expr, ibis::bitvector \&hits) const

To evaluate the exact hits.

- fuzz (const ibis::column $*$ c, ibis::fileManager::storage $*$ st, uint32_t offset=8)

The leading portion of the index file is the same as ibis::relic, which allows the constructor of the base class to work properly.

- fuzz (const ibis::column $* \mathrm{c}=0$, const char $* \mathrm{f}=0$ )

- virtual const char $*$ name () const

Returns the name of the index, similar to the function type, but returns a string instead.

- virtual void print (std::ostream \&out) const

Prints human readable information.

- virtual void read (ibis::fileManager::storage $*$ st)

Reconstructs an index from an array of bytes.

- virtual void read (const char $*$ idxfile)

Reconstructs an index from the named file.

- virtual INDEX_TYPE type () const

Returns an index type identifier.

- virtual void write (const char $* \mathrm{dt}$ ) const

Save index to a file.

\section{Protected Member Functions}

- virtual void clear ()

Clear the existing content. 


\subsubsection{Detailed Description}

The two-level interval-equality code.

Note:

In fuzzy classification / clustering, many interval equality conditions are used. Hence the crazy name.

\subsubsection{Constructor \& Destructor Documentation}

\subsubsection{1 ibis::fuzz::fuzz (const ibis::column $* \boldsymbol{c}$, ibis::fileManager::storage $* \boldsymbol{s t}$, uint32_t $s t a r t=8$ )}

The leading portion of the index file is the same as ibis::relic, which allows the constructor of the base class to work properly.

The content following the last bitvector in ibis::relic is as follows, writeCoarse.

nc (uint32_t ) - number of coarse bins. cbounds (unsigned[nc+1]) - boundaries of the coarse bins. coffsets(int32_t [ncceil(nc/2)+2]) - starting positions. cbits (bitvector[nc-ceil(nc/2)+1]) - bitvectors.

\subsubsection{Member Function Documentation}

3.54.3.1 long ibis::fuzz::evaluate (const ibis::qContinuousRange \& expr, ibis::bitvector \& hits) const [virtual]

To evaluate the exact hits.

On success, return the number of hits, otherwise a negative value is returned.

Reimplemented from ibis::relic.

\subsubsection{2 void ibis::fuzz::print (std::ostream \& out) const [ virtual ]}

Prints human readable information.

Outputs information about the index as text to the specified output stream.

Reimplemented from ibis::relic.

\subsubsection{3 void ibis::fuzz::read (ibis::fileManager::storage $* \boldsymbol{s} \boldsymbol{t}$ ) [virtual]}

Reconstructs an index from an array of bytes.

Intended for internal use only!

Reimplemented from ibis::relic.

\subsubsection{4 void ibis::fuzz::read (const char $*$ idxfile) $\quad$ [virtual]}

Reconstructs an index from the named file.

The name can be the directory containing an index file. In this case, the name of the index file must be the name of the column followed by ".idx" suffix.

Reimplemented from ibis::relic.

\subsubsection{5 void ibis::fuzz::write (const char $* \boldsymbol{d t}$ ) const [ virtual]}

Save index to a file.

Outputs the index in a compact binary format to the named file or directory. The index file contains a header that can be identified by the function isIndex. 
Reimplemented from ibis::relic.

The documentation for this class was generated from the following files:

- irelic.h

- ixfuzz.cpp

\subsection{5 ibis::horometer Class Reference}

Horometer - a primitive timing instrument.

\#include <horometer.h>

\section{Public Member Functions}

- double CPUTime () const

Return the CPU time in seconds.

- double realTime () const

Return the elapsed time in seconds.

- void resume ()

- void start ()

- void stop ()

\subsubsection{Detailed Description}

Horometer - a primitive timing instrument.

This is intented to be a simple timer. It must be explicitly started by calling function start. The same function start may be called to restart the timer which will discard previously marked starting point. The function stop must be called before functions realTime and CPUTime can report the correct time. After a horometer is stopped, it may restart by calling start, or it may resume timing by calling resume.

Timing accuracy depends on the underlying implementation. On most unix systems, the time resolution is about 0.01 seconds. The timing function itself may take $\sim 10,000$ clock cycles to execute, which is about 30 microseconds on a $400 \mathrm{MHz}$ machine.

\subsubsection{Member Function Documentation}

\subsubsection{1 void ibis::horometer::resume () [inline ]}

3.55.2.2 void ibis::horometer::start () [inline ]

\subsubsection{3 void ibis::horometer::stop () [inline ]}

The documentation for this class was generated from the following file:

- horometer.h 


\subsection{6 ibis::index Class Reference}

The base index class.

\#include <index.h>

Inheritance diagram for ibis::index::

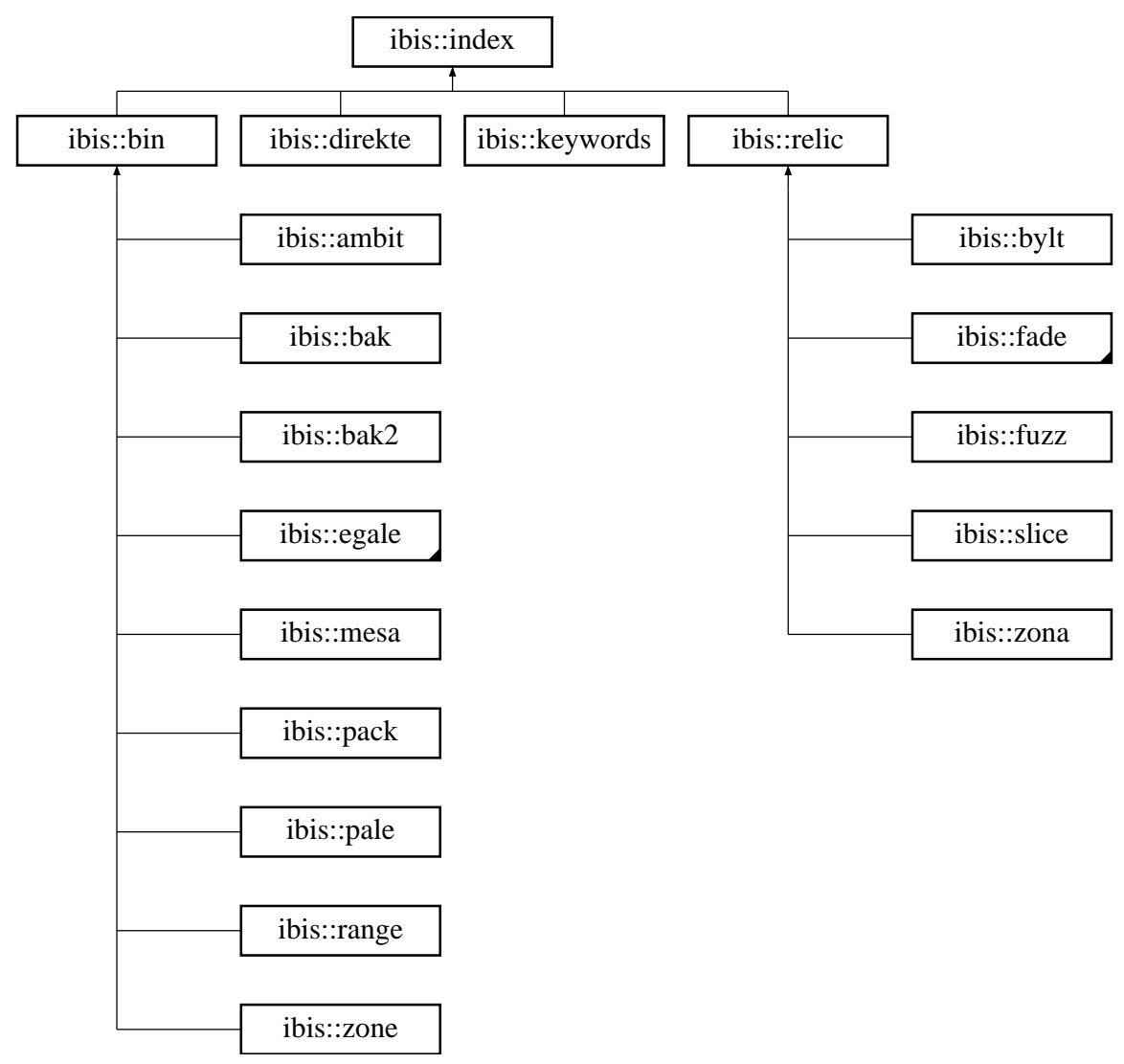

\section{Public Types}

- typedef std::map $<$ double, uint32_t $>$ histogram

- enum INDEX_TYPE \{

BINNING $=0$, RANGE, MESA, AMBIT,

PALE, PACK, ZONE, RELIC,

ROSTER, SLICE, FADE, SBIAD,

SAPID, EGALE, MOINS, ENTRE,

BAK, BAK2, KEYWORDS, MESH,

BAND, DIREKTE, GENERIC, BYLT,

FUZZ, ZONA \}

- typedef std::map $<$ double, ibis::bitvector $*>$ VMap

\section{Public Member Functions}

- virtual long append (const char $* \mathrm{dt}$, const char $* \mathrm{df}$, uint32_t nnew) $=0$

Extend the index. 
- virtual void binBoundaries (std::vector $<$ double $>\&$ ) const $=0$

The function binBoundaries and binWeights return bin boundaries and counts of each bin respectively.

- virtual void binWeights (std::vector $<$ uint32_t $>\&$ ) const $=0$

- virtual int contractRange (ibis::qContinuousRange \&rng) const

- virtual int64_t estimate (const ibis::rangeJoin \&expr, const ibis::bitvector \&mask, const ibis::qRange $*$ const range1, const ibis::qRange $*$ const range2) const

- virtual void estimate (const ibis::rangeJoin \&expr, const ibis::bitvector \&mask, const ibis::qRange $*$ const range1, const ibis::qRange *const range2, ibis::bitvector64 \&lower, ibis::bitvector64 \&upper) const

Evaluating a join condition with one (likely composite) index.

- virtual int64_t estimate (const ibis::index \&idx2, const ibis::rangeJoin \&expr, const ibis::bitvector \&mask, const ibis::qRange $*$ const range1, const ibis::qRange $*$ const range2) const

- virtual int64_t estimate (const ibis::index \&idx2, const ibis::rangeJoin \&expr, const ibis::bitvector \&mask) const

Estimate an upper bound for the number of pairs produced from marked records.

- virtual int64_t estimate (const ibis::index \&idx2, const ibis::rangeJoin \&expr) const

Estimate an upper bound for the number of pairs.

- virtual void estimate (const ibis::index \&idx2, const ibis::rangeJoin \&expr, const ibis::bitvector \&mask, const ibis::qRange $*$ const range1, const ibis::qRange $*$ const range2, ibis::bitvector64 \&lower, ibis::bitvector64 \&upper) const

- virtual void estimate (const ibis::index \&idx2, const ibis::rangeJoin \&expr, const ibis::bitvector \&mask, ibis::bitvector64 \&lower, ibis::bitvector64 \&upper) const

Estimate the pairs for the range join operator.

- virtual void estimate (const ibis::index \&idx2, const ibis::rangeJoin \&expr, ibis::bitvector64 \&lower, ibis::bitvector64 \&upper) const

Estimate the pairs for the range join operator.

- virtual uint32_t estimate (const ibis::qDiscreteRange \&expr) const

- virtual void estimate (const ibis::qDiscreteRange \&expr, ibis::bitvector \&lower, ibis::bitvector \&upper) const Estimate the hits for discrete ranges, i.e., those translated from 'a IN (x, y, .

- virtual uint32_t estimate (const ibis::qContinuousRange \&expr) const=0

Returns an upper bound on the number of hits.

- virtual void estimate (const ibis::qContinuousRange \&expr, ibis::bitvector \&lower, ibis::bitvector \&upper) const $=0$

Computes an approximation of hits as a pair of lower and upper bounds.

- virtual double estimateCost (const ibis::qDiscreteRange \&expr) const=0

- virtual double estimateCost (const ibis::qContinuousRange \&expr) const=0

Estimate the code of evaluate a range condition.

- virtual long evaluate (const ibis::qContinuousRange \&expr, ibis::bitvector \&hits) const $=0$

To evaluate the exact hits.

- virtual int expandRange (ibis::qContinuousRange \&rng) const

The functions expandRange and contractRange expands or contracts the boundaries of a range condition so that the new range will have exact answers using the function estimate.

- virtual const ibis::bitvector $*$ getBitvector (uint32_t i) const 
Return a pointer to the ith bitvector used in the index (may be 0).

- virtual long getCumulativeDistribution (std::vector $<$ double $>$ \&bds, std::vector $<$ uint32_t $>$ \&cts) const

Cumulative distribution of the data.

- virtual long getDistribution (std::vector $<$ double $>$ \&bbs, std::vector $<$ uint32_t $>$ \&cts) const

Binned distribution of the data.

- virtual double getMax () const $=0$

The maximum value recorded in the index.

- virtual double getMin () const=0

The minimum value recorded in the index.

- virtual uint32_t getNRows () const

- virtual double getSum () const $=0$

Compute the approximate sum of all the values indexed.

- virtual const char $*$ name () const=0

Returns the name of the index, similar to the function type, but returns a string instead.

- virtual uint32_t numBitvectors () const

Returns the number of bit vectors used by the index.

- virtual void print (std::ostream \&out) const=0

Prints human readable information.

- virtual void read (ibis::fileManager::storage $*$ st $)=0$

Reconstructs an index from an array of bytes.

- virtual void read (const char $*$ name) $=0$

Reconstructs an index from the named file.

- virtual void speedTest (std::ostream \&out) const

Time some logical operations and print out their speed.

- virtual INDEX_TYPE type () const=0

Returns an index type identifier.

- virtual float undecidable (const ibis::qDiscreteRange \&expr, ibis::bitvector \&iffy) const

- virtual float undecidable (const ibis::qContinuousRange \&expr, ibis::bitvector \&iffy) const $=0$

Mark the position of the rows that can not be decided with this index.

- virtual void write (const char $*$ name) const $=0$

Save index to a file.

- virtual $\sim$ index ()

The destructor. 


\section{Static Public Member Functions}

- static ibis::index $*$ create (const ibis::column $*$ c, const char $*$ name $=0$, const char $*$ spec $=0$ )

Index factory.

- static void divideCounts (array_t $<$ uint32_t $>$ \&bounds, const array_t $<$ uint32_t $>$ \&cnt)

Determine how to split the array $\mathrm{cnt}$, so that each group has roughly the same total value.

- static bool isIndex (const char $* \mathrm{f}$, INDEX_TYPE t)

Read the header of the named file to determine if it contains an index of the specified type.

- template $<$ typename E1, typename E2 $>$ static void mapValues (const array_t $<$ E1 $>$ \&val1, const array_t $<$ E2 $>$ \&val2, array_t $<$ E1 $>$ \&bnd1, array_t $<$ E2 $>$ \&bnd2, std::vector $<$ uint32_t $>$ \&cnts)

Compute a two-dimensional histogram.

- template $<$ typename $\mathrm{E}>$ static void mapValues (const array_t $<\mathrm{E}>$ \& val, array_t $<\mathrm{E}>$ \&bounds, std::vector $<$ uint32_t $>\&$ cnts)

- template $<$ typename $\mathrm{E}>$ static void mapValues (const array_t $<\mathrm{E}>$ \&val, histogram \&hist, uint32_t count=0)

- template $<$ typename $\mathrm{E}>$ static void mapValues (const array_t $<\mathrm{E}>$ \&val, VMap \&bmap)

\section{Protected Member Functions}

- virtual void activate (uint32_t i, uint32_t j) const

Regenerate bitvectors $i$ (inclusive) through $j$ (exclusive) from the underlying storage.

- virtual void activate (uint32_t i) const

Regenerate the ith bitvector from the underlying storage.

- virtual void activate () const

Regenerate all bitvectors from the underlying storage.

- void addBits (uint32_t ib, uint32_t ie, ibis::bitvector \&res, const ibis::bitvector \&tot) const

Compute the sum of bit vectors $[\mathrm{ib}, \mathrm{ie})$.

- void addBits (uint32_t ib, uint32_t ie, ibis::bitvector \&res) const

Add the sum of bits[ib] through bits[ie-1] to res.

- virtual void clear ()

Clear the existing content.

- void computeMinMax (const char $*$ f, double \&min, double \&max) const

- void dataFileName (const char $*$ f, std::string \&name) const

Generate data file name from " $f$ ".

- index (const ibis::column $* \mathrm{c}=0$, ibis::fileManager::storage $* \mathrm{~s}=0$ )

Protect the constructors so that ibis::index can not be instantiated directly.

- void indexFileName (const char $*$ f, std::string \&name) const

Generate index file name from " $f$ ".

- void mapValues (const char $* \mathrm{f}$, histogram \&hist, uint32_t count=0) const

Generate a histogram. 
- void mapValues (const char $*$ f, VMap \&bmap) const

Map the positions of each individual value.

- void optionalUnpack (std::vector $<$ ibis::bitvector $*>$ \&bits, const char $*$ opt)

A function to decide whether to uncompress the bitvectors.

- void sumBits (uint32_t ib, uint32_t ie, ibis::bitvector \&res, uint32_t ib0, uint32_t ie0) const

Compute a new sum for bit vectors [ib, ie) by taking advantage of the old sum for bitvectors [ib0, ieO).

- void sumBits (uint32_t ib, uint32_t ie, ibis::bitvector \&res) const

Compute the bitwise OR of all bitvectors (in bits) from ib to ie.

\section{Static Protected Member Functions}

- static void addBins (const std::vector $<$ ibis::bitvector $*>$ \&bits, uint32_t ib, uint32_t ie, ibis::bitvector \&res) Add the bts[ib:ie-1] to res.

- static void indexFileName (std::string \&name, const ibis::column $*$ col1, const ibis::column $*$ col2, const char $* \mathrm{f}=0$ )

Generate the index file name for the composite index fromed on two columns.

- static void setBases (array_t $<$ uint32_t $>$ \&bases, uint32_t card, uint32_t nbase=2)

Fill the array bases with the values that cover the range $[0$, card).

- static void sumBins (const std::vector $<$ ibis::bitvector $*>$ \&bits, const ibis::bitvector \& tot, uint32_t ib, uint32_t ie, ibis::bitvector \&res)

Sum up bts [ib:ie-1] and add the result to res.

- static void sumBins (const std::vector $<$ ibis::bitvector $*>$ \&bits, uint32_t ib, uint32_t ie, ibis::bitvector \&res)

Sum up bts[ib:ie-1] and place the result in res.

\section{Protected Attributes}

- std::vector $<$ ibis::bitvector $*>$ bits

A list of bitvectors.

- const ibis::column $*$ col

Pointer to the column this index is for.

- const char $*$ fname

The name of the file containing the index.

- uint32_t nrows

The number of rows represented by the index.

- $\operatorname{array} \_\mathrm{t}<$ int32_t $>$ offsets

Starting positions of the bitvectors.

- ibis:::fileManager::storage $*$ str

The underlying storage. 


\section{Classes}

- class barrel

A specialization that adds function setValue.

\subsubsection{Detailed Description}

The base index class.

Class ibis::index contains the common definitions and virtual functions of the class hierarchy. It is assumed that an ibis::index is for only one column. The user is to create an new index through the function ibis::index::create and only use the functions defined in this class.

\subsubsection{Constructor \& Destructor Documentation}

3.56.2.1 ibis::index::index (const ibis::column $* c=0$, ibis::fileManager::storage $* s=0$ ) [in]ine, protected]

Protect the constructors so that ibis::index can not be instantiated directly.

It already can not be instantiated because some functions are pure virtual, but this also reduces the size of public interface.

\subsubsection{Member Function Documentation}

3.56.3.1 void ibis::index::addBins (const std::vector $<$ ibis::bitvector $*>\&$ bts, uint32_t $i b$, uint32_t $i e$, ibis::bitvector \& res) [static, protected]

Add the bts[ib:ie-1] to res.

Since the set of bit vectors are explicitly given, there is no need to perform activation. To minimize the burden of deciding which bit vectors to activate, this function always use the bt s[ib] through bts[ie-1].

Note:

The caller need to activate the bit vectors!

This function still has to check whether a particular bts[i] is a null pointer before using the bit vector.

3.56.3.2 void ibis::index::addBits (uint32_t $i b$, uint32_t ie, ibis::bitvector $\&$ res, const ibis::bitvector $\&$ tot) const [protected]

Compute the sum of bit vectors $[i b, i e)$.

If computing a complement is faster, assume all bit vectors add up to tot.

3.56.3.3 void ibis::index::addBits (uint32_t $i b$, uint32_t $i e$, ibis::bitvector $\&$ res) const [protected]

Add the sum of bits[ib] through bits[ie-1] to res.

Always explicitly use bits[ib] through bits[ie-1].

3.56.3.4 ibis::index $*$ ibis: :index::create (const ibis::column $* c$, const char $*$ name $=0$, const char $*$ spec $=0$ ) [static]

Index factory.

It creates a specific concrete index object. If this function failed to read a specified index file, it will attempt to create a new index based on the current data file and index specification. The new index will be written under the old name. 
This function returns nil if it fails to create an index.

\section{Parameters:}

$c$ a pointer to a ibis::column object. This argument must be present.

name a name, it can be the name of the index file, the data file, or the directory containing the data file. If the name ends with '.idx' is treated as an index file and the content of the file is read. If the name does not end with '.idx', it is assumed to be the data file name unless it is determined to be directory name. If it is a directory name, the data file is assumed to be in the directory with the file name same as the column name. Once a data file is found, the content of the data file is read to construct a new index according to the return value of function indexSpec. The argument name can be nil, in which case, the data file name is constructed by concatenate the return of dataTable()->currentDataDir() and the column name.

Note:

Set name to null to build a brand new index and discard the existing index.

\section{Parameters:}

spec the index specification. This string contains the parameters for how to create an index. The most general form is

$$
\text { <binning } \ldots / \text { / <encoding } \ldots / \text { / <compression } \ldots / \text { / }
$$

Here is one example (it is the default for some integer columns)

<binning none /> <encoding equality />

FastBit always compresses every bitmap it ever generates. The compression option is to instruct it to uncompress some bitmaps or not compress indices at all. The compress option is usually not used.

If the argument spec is not specified, this function checks the specification in the following order.

1. use the index specification for the column being indexed;

2. use the index specification for the table containing the column being indexed;

3. use the most specific index specification relates to the column be indexed in the global resources (gParameters).

It stops looking as soon as it finds the first non-empty string. To override any general index specification, one must provide a complete index specification string.

3.56.3.5 void ibis::index::divideCounts (array_t $<$ uint32_t $>\&$ bdry, const array_t $<$ uint32_t $>\&$ cnt) [static]

Determine how to split the array cnt, so that each group has roughly the same total value.

The first group is [0, bdry[0]), the second group is [bdry[0], bdry[1]), and so on. Ths function uses the size of array bdry to determine the number of groups to use.

3.56.3.6 void ibis::index::estimate (const ibis::index \& idx2, const ibis::rangeJoin \& expr, const ibis::bitvector \& mask, ibis::bitvector64 \& lower, ibis::bitvector64 \& upper) const [virtual]

Estimate the pairs for the range join operator.

Only records that are masked are evaluated. 
3.56.3.7 void ibis::index::estimate (const ibis::qDiscreteRange \& expr, ibis::bitvector \& lower, ibis::bitvector \& upper) const [virtual]

Estimate the hits for discrete ranges, i.e., those translated from 'a IN (x, y, .

.)'.

Reimplemented in ibis::direkte, and ibis::relic.

3.56.3.8 virtual void ibis::index::estimate (const ibis::qContinuousRange \& expr, ibis::bitvector \& lower, ibis::bitvector \& upper) const [pure virtual]

Computes an approximation of hits as a pair of lower and upper bounds.

\section{Parameters:}

expr the query expression to be evaluated.

lower a bitvector marking a subset of the hits. All rows marked with one (1) are definitely hits.

upper a bitvector marking a superset of the hits. All hits are marked with one, but some of the rows marked one may not be hits. If the variable upper is empty, the variable lower is assumed to contain the exact answer.

Implemented in ibis::bin, ibis::range, ibis::mesa, ibis::ambit, ibis::pale, ibis::pack, ibis::zone, ibis::egale, ibis::moins, ibis::entre, ibis::direkte, ibis::keywords, ibis::relic, and ibis::slice.

3.56.3.9 virtual long ibis::index::evaluate (const ibis::qContinuousRange \& expr, ibis::bitvector \& hits) const [pure virtual]

To evaluate the exact hits.

On success, return the number of hits, otherwise a negative value is returned.

Implemented in ibis::bin, ibis::range, ibis::mesa, ibis::ambit, ibis::pale, ibis::pack, ibis::zone, ibis::egale, ibis::moins, ibis::entre, ibis::direkte, ibis::keywords, ibis::relic, ibis::slice, ibis::fade, ibis::sbiad, ibis::sapid, ibis::fuzz, ibis::bylt, and ibis::zona.

\subsubsection{0 virtual int ibis::index::expandRange (ibis::qContinuousRange \& rng) const [inline, virtual]}

The functions expandRange and contractRange expands or contracts the boundaries of a range condition so that the new range will have exact answers using the function estimate.

The default implementation provided does nothing since this is only meaningful for indices based on bins.

Reimplemented in ibis::bin, ibis::range, ibis::bak, and ibis::bak2.

\subsubsection{1 virtual double ibis::index::getSum () const [pure virtual]}

Compute the approximate sum of all the values indexed.

If it decides that computing the sum directly from the vertical partition is more efficient, it will return NaN immediately.

Implemented in ibis::bin, ibis::range, ibis::mesa, ibis::ambit, ibis::pack, ibis::egale, ibis::moins, ibis::entre, ibis::direkte, ibis::keywords, ibis::relic, ibis::slice, and ibis::fade.

3.56.3.12 void ibis::index::indexFileName (std::string \& iname, const ibis::column $*$ col1, const ibis::column $* \boldsymbol{c o l} 2$, const char $* \boldsymbol{d i r}=0) \quad$ [static, protected]

Generate the index file name for the composite index fromed on two columns.

May use argument "dir" if it is not null. 


\subsubsection{3 bool ibis::index::isIndex (const char $* \boldsymbol{f}$, INDEX_TYPE $\boldsymbol{t}$ ) [ static ]}

Read the header of the named file to determine if it contains an index of the specified type.

Returns true if the correct header is found, else return false.

3.56.3.14 void ibis::index::mapValues (const char $* f$, histogram \& hist, uint32_t count $=0$ ) const [protected]

Generate a histogram.

A value of any supported type is supposed to be able to fit in a double with no rounding, no approximation and no overflow.

\subsubsection{5 void ibis::index::mapValues (const char $* \boldsymbol{f}$, VMap \& $\boldsymbol{b m a p}$ ) const [protected]}

Map the positions of each individual value.

Given a file containing the values of a column, this function maps the position of each individual values and stores the result in a set of bitmaps.

Note:

IMPROTANT ASSUMPTION. A value of any supported type is supposed to be able to fit in a double with no rounding, no approximation and no overflow.

3.56.3.16 template $<$ typename E1, typename E2 $>$ void ibis::index::mapValues (const array_t $<$ E1 $>\&$ val1, const array_t $<$ E2 $>\&$ val2, array_t $<$ E1 $>$ \& bnd1, array_t $<$ E2 $>$ \& bnd2, std::vector $<$ uint32_t $>$ \& cnts) [static]

Compute a two-dimensional histogram.

Given two arrays of the same size, count the number of appearance of each combinations defined by bnd1 and bnd2. If the arrays bnd1 or bnd2 contain values in ascending order, their values are directly used in this function. The empty one will be replaced by a linear division of actual range into 256 bins. The array counts stores the 2-D bins in raster-scan order with the second variable, val2, as the faster varying variables. More specifically, the bins for variable 1 are defined as:

$/ / /(\ldots$, bnd1 [0] ) [bnd1[1], bin1[2]) [bnd1[2], bin1[3) .. [bnd1.back(), ..)

$/ / /$

Note that '[' denote the left boundary is inclusive and ')' denote the right boundary is exclusive. Similarly, the bins for variable 2 are

$/ / /(\ldots$, bnd2 [0] ) [bnd2[1], bin2[2]) [bnd2[2], bin2[3) .. [bnd2.back(), ..)

///

The counts are for the following bins

$/ / /(\ldots, \operatorname{bin} 1[0])(\ldots \operatorname{bin} 2[0])$

$/ / /(\ldots, \operatorname{bin} 1[0])$ [bin2[0], bin2[1])

$/ / /(\ldots$, bin1[0]) [bin2[1], bin2[2])

$/ / / \ldots$

$/ / /(\ldots, \operatorname{bin} 1[0])$ [bin2.back(), ...)

$/ / /$ [bin1[0], bin1[1]) (.., bin2[0])

$/ / /$ [bin1[0], bin1[1]) [bin2[0], bin2[1])

$/ / /$ [bin1[0], bin1[1]) [bin2[1], bin2[2])

$/ / / \ldots$

$/ / /[\operatorname{bin} 1[0], \operatorname{bin} 1[1])$ [bin2.back(), ...)

$/ / / \ldots$

$/ / /$ 


\subsubsection{7 virtual void ibis::index::print (std::ostream \& out) const [pure virtual]}

Prints human readable information.

Outputs information about the index as text to the specified output stream.

Implemented in ibis::bin, ibis::range, ibis::mesa, ibis::ambit, ibis::pale, ibis::pack, ibis::zone, ibis::egale, ibis::moins, ibis::entre, ibis::bak, ibis::bak2, ibis::direkte, ibis::keywords, ibis::relic, ibis::slice, ibis::fade, ibis::sbiad, ibis::sapid, ibis::fuzz, ibis::bylt, and ibis::zona.

\subsubsection{8 virtual void ibis::index::read (ibis::fileManager::storage $* \boldsymbol{s t}$ ) [pure virtual]}

Reconstructs an index from an array of bytes.

Intended for internal use only!

Implemented in ibis::bin, ibis::range, ibis::ambit, ibis::pale, ibis::pack, ibis::zone, ibis::egale, ibis::direkte, ibis::keywords, ibis::relic, ibis::slice, ibis::fade, ibis::fuzz, ibis::bylt, and ibis::zona.

\subsubsection{9 virtual void ibis::index::read (const char $*$ name) [pure virtual]}

Reconstructs an index from the named file.

The name can be the directory containing an index file. In this case, the name of the index file must be the name of the column followed by ".idx" suffix.

Implemented in ibis::bin, ibis::range, ibis::ambit, ibis::pale, ibis::pack, ibis::zone, ibis::egale, ibis::bak, ibis::bak2, ibis::direkte, ibis::keywords, ibis::relic, ibis::slice, ibis::fade, ibis::fuzz, ibis::bylt, and ibis::zona.

3.56.3.20 void ibis::index::setBases (array_t $<$ uint32_t $>\&$ bases, uint32_t card, uint32_t nbase $=2$ ) [static, protected]

Fill the array bases with the values that cover the range $[0$, card).

Assumes at least two components. For one component case use indices defined explicit for one component cases.

3.56.3.21 void ibis::index::sumBins (const std::vector $<$ ibis::bitvector $*>\&$ bts, const ibis::bitvector \& tot, uint32_t $\boldsymbol{i} \boldsymbol{b}$, uint32_t $\boldsymbol{i e}$, ibis: :bitvector \& res) [ static, protected]

Sum up bts[ib:ie-1] and add the result to res.

It is assumed that all bts add up to tot. In the other version of sumBins without this argument tot, it was assumed that all bitmaps add up to a bit vector of all ones. The decision of whether to use bts[ib:ie-1] directly or use the subtractive version (using bts[0:ib-1] and bts[ie:nobs-1]) are based on the number of bit vectors.

3.56.3.22 void ibis::index::sumBins (const std::vector $<$ ibis::bitvector $*>\& b t s$, uint32_t $i b$, uint32_t $i e$, ibis::bitvector \& res) [static, protected]

Sum up bts [ib:ie-1] and place the result in res.

Note:

This function may either use bts[ib:ie-1] or bts[0:ib-1] and bts[ie:nobs-1] depending which set has more bit vectors! This requires the caller to activate the appropriate set.

This function always uses the operator $\mid=$. Tests show that using the function setBit is always slower.

3.56.3.23 void ibis::index::sumBits (uint32_t $i b$, uint32_t $i e$, ibis::bitvector $\&$ res, uint32_t $i b 0$, uint32_t ie0) const [protected]

Compute a new sum for bit vectors [ib, ie) by taking advantage of the old sum for bitvectors [ib0, ie0). 
- On input, res $=\operatorname{sum}_{-}\{\mathrm{i}=\mathrm{ib} 0\}^{\wedge}\{\mathrm{ie} 0\}$ bits $[\mathrm{i}]$.

- On exit, res $=\operatorname{sum}_{-}\{\mathrm{i}=\mathrm{ib}\}^{\wedge}\{\mathrm{ie}\}$ bits[i].

\subsubsection{4 void ibis::index::sumBits (uint32_t $i b$, uint32_t $i e$, ibis::bitvector $\&$ res) const [protected]}

Compute the bitwise OR of all bitvectors (in bits) from ib to ie.

As usual, bits[ib] is included but bits[ie] is excluded.

3.56.3.25 virtual float ibis::index::undecidable (const ibis::qContinuousRange \& expr, ibis::bitvector \& iffy) const [pure virtual]

Mark the position of the rows that can not be decided with this index.

\section{Parameters:}

expr the range conditions to be evaluated.

iffy the bitvector marking the positions of rows that can not be decided using the index. Return value is the expected fraction of undecided rows that might satisfy the range conditions.

Implemented in ibis::bin, ibis::range, ibis::mesa, ibis::ambit, ibis::pale, ibis::pack, ibis::zone, ibis::egale, ibis::direkte, ibis::keywords, and ibis::relic.

\subsubsection{6 virtual void ibis::index::write (const char $*$ name) const [pure virtual]}

Save index to a file.

Outputs the index in a compact binary format to the named file or directory. The index file contains a header that can be identified by the function isIndex.

Implemented in ibis::bin, ibis::range, ibis::mesa, ibis::ambit, ibis::pale, ibis::pack, ibis::zone, ibis::egale, ibis::moins, ibis::entre, ibis::bak, ibis::bak2, ibis::direkte, ibis::keywords, ibis::relic, ibis::slice, ibis::fade, ibis::sbiad, ibis::sapid, ibis::fuzz, ibis::bylt, and ibis::zona.

\subsubsection{Member Data Documentation}

\subsubsection{1 ibis::fileManager::storage $*$ ibis::index::str [mutable, protected]}

The underlying storage.

It may be nil if bitvectors are not from a storage object managed by the file manager.

The documentation for this class was generated from the following files:

- index.h

- index.cpp

\subsection{7 ibis::index::barrel Class Reference}

A specialization that adds function setValue.

\#include <index.h>

\section{Public Member Functions}

- barrel (const ibis::compRange::term $* \mathrm{t}$ )

- void setValue (uint32_t $i$, double v) 


\subsubsection{Detailed Description}

A specialization that adds function setValue.

This function allows the client to directly set the value for an individual variable.

The documentation for this class was generated from the following file:

- index.h

\subsection{8 ibis::keywords Class Reference}

Class ibis: : keywords defines a boolean term-document matrix.

\#include <ikeywords.h>

Inheritance diagram for ibis::keywords::

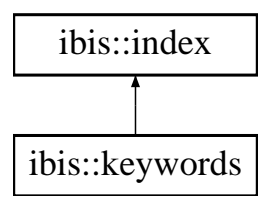

\section{Public Member Functions}

- virtual long append (const char $* \mathrm{dt}$, const char $* \mathrm{df}$, uint32_t nnew)

Extend the index.

- virtual void binBoundaries (std::vector $<$ double $>\& b$ ) const

The function binBoundaries and binWeights return bin boundaries and counts of each bin respectively.

- virtual void binWeights (std::vector $<$ uint32_t $>\& b$ ) const

- virtual uint32_t estimate (const ibis::qContinuousRange \&expr) const

Returns an upper bound on the number of hits.

- virtual void estimate (const ibis::qContinuousRange \&expr, ibis::bitvector \&lower, ibis::bitvector \&upper) const

Computes an approximation of hits as a pair of lower and upper bounds.

- virtual double estimateCost (const ibis::qDiscreteRange \&expr) const

- virtual double estimateCost (const ibis::qContinuousRange \&expr) const

Estimate the code of evaluate a range condition.

- virtual long evaluate (const ibis::qContinuousRange \&expr, ibis::bitvector \&hits) const

To evaluate the exact hits.

- virtual double getMax () const

The maximum value recorded in the index.

- virtual double getMin () const

The minimum value recorded in the index.

- virtual double getSum () const

Compute the approximate sum of all the values indexed. 
- keywords (const ibis::column $*$ c, ibis::fileManager::storage $*$ st, uint32_t offset=8)

- keywords (const ibis::column $* \mathrm{c}$, const ibis::column $* \mathrm{idcol}=0$, const char $* \mathrm{f}=0$ )

Constructor.

- virtual const char $*$ name () const

Returns the name of the index, similar to the function type, but returns a string instead.

- virtual void print (std::ostream \&out) const

Prints human readable information.

- virtual void read (ibis::fileManager::storage $*$ st)

Reconstructs an index from an array of bytes.

- virtual void read (const char $*$ idxfile)

Reconstructs an index from the named file.

- long search (const char $* \mathrm{kw}$ ) const

Estimate the number of matches.

- long search (const char $* \mathrm{kw}$, ibis::bitvector \&hits) const

Match a particular keyword.

- virtual INDEX_TYPE type () const

Returns an index type identifier.

- virtual float undecidable (const ibis::qContinuousRange \&expr, ibis::bitvector \&iffy) const

This class and its derived classes should produce exact answers, therefore no undecidable rows.

- virtual void write (const char $* \mathrm{dt}$ ) const

Write the boolean term-document matrix as two files, xxx.terms for the terms and xxx.idx for the bitmaps that marks the positions.

\section{Protected Member Functions}

- void clear ()

Clear the current content.

- char readKeyword (const char $* \&$ buf, std::string \&key) const

Extract the keyword from a line of input term-document file.

- int readLine (std::istream \&in, std::string \&key, std::vector $<$ uint32_t $>$ \&idlist, char $*$ buf, uint32_t nbuf) const

Read and process one line from the term-docuement file.

- int readTermDocFile (const ibis::column $*$ idcol, const char $* \mathrm{f}$ )

Reads a term-document list from a external file.

- uint32_t readUInt (const char $* \&$ buf) const

Extract the next integer in an inputline.

- void setBits (std::vector $<$ uint32_t $>$ \&pos, ibis::bitvector \&bvec) const 


\subsubsection{Detailed Description}

Class ibis: : keywords defines a boolean term-document matrix.

The terms are stored in an ibis: : dictionary and the bits are stored in a series of bitvectors.

The current implementation requires a .tdlist file alongside the binary string values. In addition, it uses an entry in the ibis.rc file of the following form to specify the ids used in the .tdlist file.

$<$ table-name $>$. $<$ column-name $>$.docIDName $=<$ id-column-name $>$

For example,

enrondata.subject.docIDName $=$ mid enrondata.body.docIDName $=$ mid

If an ID column is not specified, the integer IDs in the .tdlist file is assumed to the row number.

\subsubsection{Constructor \& Destructor Documentation}

\subsubsection{1 ibis::keywords::keywords (const ibis::column $* \boldsymbol{c}$, const ibis::column $*$ idcol $=0$, const char $* f=0$ )}

Constructor.

It first tries to read the terms (.terms) and the tdmat (.idx) files if they both exist. If that fails, it tries to read the tdlist (.tdlist) file generated by another program and

\subsubsection{Member Function Documentation}

3.58.3.1 void ibis::keywords::estimate (const ibis::qContinuousRange \& expr, ibis::bitvector \& lower, ibis::bitvector \& upper) const [virtual]

Computes an approximation of hits as a pair of lower and upper bounds.

\section{Parameters:}

expr the query expression to be evaluated.

lower a bitvector marking a subset of the hits. All rows marked with one (1) are definitely hits.

upper a bitvector marking a superset of the hits. All hits are marked with one, but some of the rows marked one may not be hits. If the variable upper is empty, the variable lower is assumed to contain the exact answer.

Implements ibis::index.

3.58.3.2 long ibis::keywords::evaluate (const ibis::qContinuousRange \& expr, ibis::bitvector \& hits) const [virtual]

To evaluate the exact hits.

On success, return the number of hits, otherwise a negative value is returned.

Implements ibis::index.

\subsubsection{3 virtual double ibis::keywords::getSum () const [inline, virtual]}

Compute the approximate sum of all the values indexed.

If it decides that computing the sum directly from the vertical partition is more efficient, it will return NaN immediately. Implements ibis::index. 


\subsubsection{4 void ibis::keywords::print (std::ostream \& out) const [virtual]}

Prints human readable information.

Outputs information about the index as text to the specified output stream.

Implements ibis::index.

\subsubsection{5 void ibis::keywords::read (ibis::fileManager::storage $* s t$ ) [virtual]}

Reconstructs an index from an array of bytes.

Intended for internal use only!

Implements ibis::index.

\subsubsection{6 void ibis::keywords::read (const char $*$ idxfile) [ virtual]}

Reconstructs an index from the named file.

The name can be the directory containing an index file. In this case, the name of the index file must be the name of the column followed by ".idx" suffix.

Implements ibis::index.

3.58.3.7 char ibis::keywords::readKeyword (const char $*$ \& buf, std::string \& keyword) const [in]ine, protected]

Extract the keyword from a line of input term-document file.

Returns the first non-space character after the keyword.

\subsubsection{8 int ibis::keywords::readTermDocFile (const ibis::column $* \boldsymbol{i d c o l}$, const char $* \boldsymbol{f}$ ) [protected]}

Reads a term-document list from a external file.

Returns the number of terms found if successful, otherwise returns a negative number to indicate error.

The documentation for this class was generated from the following files:

- ikeywords.h

- ikeywords.cpp

\subsection{9 ibis::lessi Struct Reference}

A case-insensitive version of less for comparing names of tables, columns, and resources.

\#include <const.h>

\section{Public Member Functions}

- bool operator() (const char $* \mathrm{x}$, const char $* \mathrm{y})$ const

\subsubsection{Detailed Description}

A case-insensitive version of less for comparing names of tables, columns, and resources.

The documentation for this struct was generated from the following file:

- const.h 


\subsection{0 ibis::mensa Class Reference}

Class ibis::mensa contains multiple (horizontal) data partitions (ibis::part) to form a logical data table.

\#include <mensa.h>

Inheritance diagram for ibis::mensa::

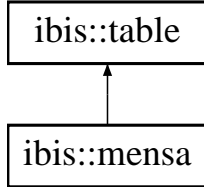

\section{Public Member Functions}

- virtual int buildIndex (const char $*$, const char $*$ )

Create the index for named column.

- virtual int buildIndexes (const char $*$ )

Create indexes for every column of the table.

- virtual stringList columnNames () const

Return column names.

- virtual typeList columnTypes () const

Return data types.

- virtual ibis::table::cursor $*$ createCursor () const

Create a cursor object to perform row-wise data access.

- virtual void describe (std::ostream \&) const

Print a description of the table to the specified output stream.

- virtual int dump (std::ostream \&, const char $*$ ) const

Dump the values in ASCII form to the specified output stream.

- virtual void estimate (const char *cond, uint64_t \&nmin, uint64_t \&nmax) const

Estimate the number of rows satisfying the selection conditions.

- virtual int64_t getColumnAsBytes (const char $*$, char $*$ ) const

Retrieve all values of the named column.

- virtual int64_t getColumnAsDoubles (const char $*$, double $*$ ) const

Note:

Integers four-byte or less in length can be converted to double safely.

- virtual int64_t getColumnAsFloats (const char $*$, float $*$ ) const

Note:

Integers two-byte or less in length can be converted safely to floats.

- virtual int64_t getColumnAsInts (const char $*$, int32_t $*$ ) const

Retrieve all values of the named column. 
- virtual int64_t getColumnAsLongs (const char $*$, int64_t $*$ ) const

Note:

All integers 4-byte or shorter in length can be safely converted into int64_t.

- virtual int64_t getColumnAsShorts (const char $*$, int16_t $*$ ) const

Retrieve all values of the named column.

- virtual int64_t getColumnAsStrings (const char $*$, std::vector $<$ std::string $>\&$ ) const

Note:

Any data type can be converted to strings, however, the conversion may take a significant amount of time.

- virtual int64_t getColumnAsUBytes (const char $*$, unsigned char $*$ ) const

Retrieve all values of the named column.

- virtual int64_t getColumnAsUInts (const char $*$, uint32_t $*$ ) const

Retrieve all values of the named column.

- virtual int64_t getColumnAsULongs (const char $*$, uint64_t $*$ ) const

Note:

All integers can be converted to uint64_t, however, negative integers will be treated as unsigned integers.

- virtual int64_t getColumnAsUShorts (const char *, uint16_t *) const

Retrieve all values of the named column.

- virtual long getHistogram (const char $*$, const char $*$, double, double, double, std::vector $<$ size_t $>\&$ ) const

Compute the histogram of the named column.

- virtual long getHistogram2D (const char $*$, const char $*$, double, double, double, const char $*$, double, double, double, std::vector $<$ size_t $>\&$ ) const

Compute a two-dimension histogram on columns cname1 and name2.

- virtual long getHistogram3D (const char $*$, const char $*$, double, double, double, const char $*$, double, double, double, const char $*$, double, double, double, std::vector $<$ size_t $>\&$ ) const

Compute a three-dimensional histogram on the named columns.

- virtual table $*$ groupby (const stringList \&) const

Directly performing group-by on the base data (without selection) is not currently supported.

- virtual void indexSpec (const char $*$, const char $*$ )

Replace the current indexing option.

- virtual const char $*$ indexSpec (const char $*$ ) const

Retrieve the current indexing option.

- mensa (const char $*$ dir1, const char $* \operatorname{dir} 2$ )

- mensa (const char $*$ dir)

- virtual size_t nColumns () const

- virtual uint64_t nRows () const

- virtual void orderby (const stringList \&)

Reordering the rows using the specified columns.

- virtual void reverseRows () 
Reversing the ordering of the rows on disk requires too much work but has no obvious benefit.

- virtual table $*$ select (const char $*$ sel, const char $*$ cond) const

Given a set of column names and a set of selection conditions, compute another table that represents the selected values.

\section{Protected Member Functions}

- template $<$ typename $\mathrm{T}>$ void addIncoreData (void $* \&$ to, const array_t $<\mathrm{T}>\&$ from, size_t nold, const $\mathrm{T}$ special) const

Append new data (in from) to a larger array (pointed to by to).

- void addStrings (void $* \&$, const std::vector $<$ std::string $>\&$, size_t $)$ const

- void clear ()

Clear the existing content.

- int64_t computeHits (const char $*$ cond) const

Compute the number of hits.

\section{Protected Attributes}

- ibis::table::namesTypes naty

A combined list of columns names.

- uint64_t nrows

- ibis::partList parts

List of data partitions.

\section{Friends}

- class cursor

\section{Classes}

- class cursor

\subsubsection{Detailed Description}

Class ibis::mensa contains multiple (horizontal) data partitions (ibis::part) to form a logical data table.

The base data contained in this table is logically immutable as reordering rows (through function reorder) does not change the overall content of the table. The functions reverseRows and groupby are not implmented.

Note:

Mensa is a Latin word for "table." 


\subsubsection{Member Function Documentation}

\subsubsection{1 int ibis::mensa::buildIndex (const char $*$, const char $*$ ) [virtual]}

Create the index for named column.

The existing index will be replaced. If an indexing option is not specified, it will use the internally recorded option for the named column or the table containing the column.

\section{Note:}

Unless any there is a specific instruction to not index a column, the querying functions will automatically build indices as necessary. However, as building an index is relatively expensive process, building an index on a column is on average about four or five times as expensive as reading the column from disk, this function is provided so that it is possible to build indexes beforehand.

Implements ibis::table.

\subsubsection{2 int ibis::mensa::buildIndexes (const char $*$ ) [virtual]}

Create indexes for every column of the table.

Existing indexes will be replaced. If an indexing option is not specified, the internally recorded options will be used. buildIndex

Implements ibis::table.

\subsubsection{3 int ibis::mensa::dump (std::ostream \&, const char $*$ ) const [virtual]}

Dump the values in ASCII form to the specified output stream.

The default delimiter is coma (","), which produces Comma-Separated-Values (CSV).

Implements ibis::table.

3.60.2.4 void ibis::mensa::estimate (const char $*$ cond, uint64_t \& nmin, uint64_t \& nmax) const [virtual]

Estimate the number of rows satisfying the selection conditions.

The number of rows is between [nmin, nmax].

Implements ibis::table.

\subsubsection{5 int64_t ibis::mensa::getColumnAsBytes (const char $*$, char $*$ ) const [virtual]}

Retrieve all values of the named column.

The member functions of this class only support access to whole column at a time. Use table: : cursor class for row-wise accesses. For fixed-width data types, the raw pointers are used to point to the values to be returned. In these cases, the caller is responsible for allocating enough storage for the values to be returned.

Implements ibis::table.

\subsubsection{6 int64_t ibis::mensa::getColumnAsDoubles (const char $*$ cn, double $*$ vals) const [virtua ] ]}

\section{Note:}

Integers four-byte or less in length can be converted to double safely.

Float values may also be converted into doubles.

Implements ibis::table. 


\subsubsection{7 int64_t ibis::mensa::getColumnAsInts (const char $*$, int32_t $*$ ) const [virtual]}

Retrieve all values of the named column.

The member functions of this class only support access to whole column at a time. Use table: : cursor class for row-wise accesses. For fixed-width data types, the raw pointers are used to point to the values to be returned. In these cases, the caller is responsible for allocating enough storage for the values to be returned.

Implements ibis::table.

\subsubsection{8 int64_t ibis::mensa::getColumnAsLongs (const char $* \boldsymbol{c n}$, int64_t $* v a l s)$ const [virtua l ]}

Note:

All integers 4-byte or shorter in length can be safely converted into int64_t.

Values in uint64_t are treated as signed integers, which may create incorrect values.

Implements ibis::table.

\subsubsection{9 int64_t ibis::mensa::getColumnAsShorts (const char $*$, int16_t $*$ ) const [virtual]}

Retrieve all values of the named column.

The member functions of this class only support access to whole column at a time. Use table: : cursor class for row-wise accesses. For fixed-width data types, the raw pointers are used to point to the values to be returned. In these cases, the caller is responsible for allocating enough storage for the values to be returned.

Implements ibis::table.

\subsubsection{0 int64_t ibis::mensa::getColumnAsUBytes (const char $*$, unsigned char $*$ ) const [virtual]}

Retrieve all values of the named column.

The member functions of this class only support access to whole column at a time. Use table: : cursor class for row-wise accesses. For fixed-width data types, the raw pointers are used to point to the values to be returned. In these cases, the caller is responsible for allocating enough storage for the values to be returned.

Implements ibis::table.

\subsubsection{1 int64_t ibis::mensa::getColumnAsUInts (const char $*$, uint32_t $*$ ) const $\quad$ [virtual]}

Retrieve all values of the named column.

The member functions of this class only support access to whole column at a time. Use table: : cursor class for row-wise accesses. For fixed-width data types, the raw pointers are used to point to the values to be returned. In these cases, the caller is responsible for allocating enough storage for the values to be returned.

Implements ibis::table.

\subsubsection{2 int64_t ibis::mensa::getColumnAsUShorts (const char $*$, uint16_t $*$ ) const [virtua I ]}

Retrieve all values of the named column.

The member functions of this class only support access to whole column at a time. Use table: : cursor class for row-wise accesses. For fixed-width data types, the raw pointers are used to point to the values to be returned. In these cases, the caller is responsible for allocating enough storage for the values to be returned.

Implements ibis::table. 
3.60.2.13 long ibis::mensa::getHistogram (const char $*$, const char $*$, double, double, double, std::vector $<$ size_t $>$ \&) const [virtual]

Compute the histogram of the named column.

This version uses the user specified bins: [begin, begin + stride) [begin + stride, begin $+2 *$ stride), .... A record is placed in bin $(\mathrm{x}$ - begin) / stride, where the first bin is bin 0 . This gives a total of

(end - begin) / stride bins.

Note:

Records (rows) outside of the range [begin, end] are not counted.

Non-positive stride is considered as an error.

If end is less than begin, an empty array counts is returned along with return value 0 .

Implements ibis::table.

3.60.2.14 long ibis::mensa::getHistogram2D (const char $*$, const char $*$, double, double, double, const char $*$, double, double, double, std::vector $<$ size_t $>$ \&) const $\quad$ [virtual]

Compute a two-dimension histogram on columns cname1 and name2.

The bins along each dimension are defined the same way as in function getHistogram. The array counts stores the two-dimensional bins with the first dimension as the slow varying dimension following $\mathrm{C}$ convention for ordering multi-dimensional arrays.

Implements ibis::table.

3.60.2.15 long ibis::mensa::getHistogram3D (const char $*$, const char $*$, double, double, double, const char $*$, double, double, double, const char $*$, double, double, double, std::vector $<$ size_t $>\&$ ) const [virtual]

Compute a three-dimensional histogram on the named columns.

The triplets $<$ begin, end, stride $>$ are used the same ways in getHistogram and getHistogram2D. The three dimensional bins are linearized in count $s$ with the first being the slowest varying dimension and the third being the fastest varying dimension following the $\mathrm{C}$ convention for ordering multi-dimensional arrays.

Implements ibis::table.

\subsubsection{6 void ibis::mensa::indexSpec (const char $*$, const char $*$ ) [virtual]}

Replace the current indexing option.

If no column name is specified, it resets the indexing option for the table.

Implements ibis::table.

\subsubsection{7 const char $*$ ibis::mensa::indexSpec (const char $*$ ) const [virtual]}

Retrieve the current indexing option.

If no column name is specified, it retrieve the indexing option for the table.

Implements ibis::table.

\subsubsection{8 void ibis::mensa::orderby (const stringList \& names) [virtual]}

Reordering the rows using the specified columns.

Each data partition is reordered separately.

Implements ibis::table.

The documentation for this class was generated from the following files: 
- mensa.h

- mensa.cpp

\subsection{1 ibis::MersenneTwister Class Reference}

Mersenne Twister generates uniform random numbers efficiently.

\#include <twister.h>

Inheritance diagram for ibis::MersenneTwister::

ibis::uniformRandomNumber

ibis::MersenneTwister

\section{Public Member Functions}

- MersenneTwister (unsigned seed)

- unsigned next ()

- unsigned next (unsigned r)

- double nextDouble ()

- float nextFloat ()

- double nextGaussian ()

- int nextInt ()

- long nextLong ()

- double nextPoisson ()

- double nextZipf (double a)

- double nextZipf ()

- virtual double operator() ()

- void setSeed (unsigned seed)

\subsubsection{Detailed Description}

Mersenne Twister generates uniform random numbers efficiently.

The documentation for this class was generated from the following file:

- twister.h

\subsection{2 ibis::mesa Class Reference}

This class implements the two-side range encoding from Chan and Ioannidis.

\#include <ibin.h>

Inheritance diagram for ibis::mesa:: 


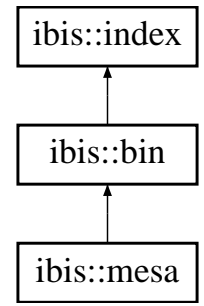

\section{Public Member Functions}

- long append (const ibis::mesa \&tail)

- virtual long append (const char $* \mathrm{dt}$, const char $* \mathrm{df}$, uint32_t nnew)

Extend the index.

- virtual void binBoundaries ( std::vector $<$ double $>\&$ ) const

The function binBoundaries and binWeights return bin boundaries and counts of each bin respectively.

- virtual void binWeights (std::vector $<$ uint32_t $>\&$ ) const

- virtual uint32_t estimate (const ibis::qContinuousRange \&expr) const

Returns an upper bound on the number of hits.

- virtual void estimate (const ibis::qContinuousRange \&expr, ibis::bitvector \&lower, ibis::bitvector \&upper) const

Computes an approximation of hits as a pair of lower and upper bounds.

- virtual long evaluate (const ibis::qContinuousRange \&expr, ibis::bitvector \&hits) const

To evaluate the exact hits.

- virtual double getSum () const

Compute the approximate sum of all the values indexed.

- mesa (const ibis::bin \&rhs)

Construct an interval encoded index from an equality encoded index.

- mesa (const ibis::column $* \mathrm{c}$, ibis::fileManager::storage $*$ st, uint32_t offset=8)

- mesa (const ibis::column $* \mathrm{c}=0$, const char $* \mathrm{f}=0$ )

- virtual const char $*$ name () const

Returns the name of the index, similar to the function type, but returns a string instead.

- virtual uint32_t numBins () const

- virtual void print (std::ostream \&out) const

Prints human readable information.

- virtual void speedTest (std::ostream \&out) const

Time some logical operations and print out their speed.

- virtual INDEX_TYPE type () const

Returns an index type identifier.

- virtual float undecidable (const ibis::qContinuousRange \&expr, ibis::bitvector \&iffy) const

Mark the position of the rows that can not be decided with this index. 
- virtual void write (const char $* \mathrm{dt})$ const Save index to a file.

\section{Protected Member Functions}

- virtual double computeSum () const

\subsubsection{Detailed Description}

This class implements the two-side range encoding from Chan and Ioannidis.

\subsubsection{Member Function Documentation}

3.62.2.1 void ibis::mesa::estimate (const ibis::qContinuousRange \& expr, ibis::bitvector \& lower, ibis::bitvector \& upper) const [virtual]

Computes an approximation of hits as a pair of lower and upper bounds.

\section{Parameters:}

expr the query expression to be evaluated.

lower a bitvector marking a subset of the hits. All rows marked with one (1) are definitely hits.

upper a bitvector marking a superset of the hits. All hits are marked with one, but some of the rows marked one may not be hits. If the variable upper is empty, the variable lower is assumed to contain the exact answer.

Reimplemented from ibis::bin.

3.62.2.2 long ibis::mesa::evaluate (const ibis::qContinuousRange \& expr, ibis::bitvector \& hits) const [virtual]

To evaluate the exact hits.

On success, return the number of hits, otherwise a negative value is returned.

Reimplemented from ibis::bin.

\subsubsection{3 double ibis::mesa::getSum () const [virtual]}

Compute the approximate sum of all the values indexed.

If it decides that computing the sum directly from the vertical partition is more efficient, it will return NaN immediately. Reimplemented from ibis::bin.

\subsubsection{4 void ibis::mesa::print (std::ostream \& out) const [virtual]}

Prints human readable information.

Outputs information about the index as text to the specified output stream.

Reimplemented from ibis::bin.

3.62.2.5 float ibis::mesa::undecidable (const ibis::qContinuousRange \& expr, ibis::bitvector \& iffy) const [virtual]

Mark the position of the rows that can not be decided with this index. 


\section{Parameters:}

expr the range conditions to be evaluated.

iffy the bitvector marking the positions of rows that can not be decided using the index. Return value is the expected fraction of undecided rows that might satisfy the range conditions.

Reimplemented from ibis::bin.

\subsubsection{6 void ibis::mesa::write (const char $* \boldsymbol{d t}$ ) const [virtual]}

Save index to a file.

Outputs the index in a compact binary format to the named file or directory. The index file contains a header that can be identified by the function isIndex.

Reimplemented from ibis::bin.

The documentation for this class was generated from the following files:

- ibin.h

- imesa.cpp

\subsection{3 ibis::meshQuery Class Reference}

The class adds more functionality to ibis::query to handle data from meshes.

\#include <meshQuery.h>

Inheritance diagram for ibis::meshQuery::

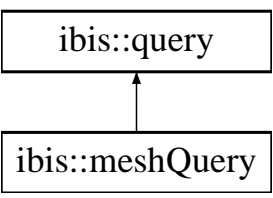

\section{Public Member Functions}

- int getHitsAsRanges (std::vector $<$ std::vector $<$ uint32_t $>>$ \&reg, const bool merge=false) const

This variant of getHitsAsRanges uses the dimensions defined by ibis::table::getMeshShape().

- int getHitsAsRanges (std::vector $<$ std::vector $<$ uint32_t $>>$ \&reg, const std::vector $<$ uint32_t $>$ \&dim, const bool merge=false) const

Translate hit vector into bounding boxes.

- int getPointsOnBoundary (std::vector $<$ std::vector $<$ uint32_t $>>$ \&bdy) const

The variant of getPointsOnBoundary use dimensions returned by ibis::table::getMeshShape().

- int getPointsOnBoundary (std::vector $<$ std::vector $<$ uint32_t $>>$ \&bdy, const std::vector $<$ uint32_t $>\&$ dim) const

Determine points with neighbors that are not hits.

- meshQuery (const char $*$ dir, const ibis::partList \&tl)

Constructor for recoverying from crash.

- meshQuery (const char $*$ uid, const part $*$ et, const char $*$ pref $=0$ )

Constructor for building a new query. 


\section{Static Public Member Functions}

- static int bitvectorToCoordinates (const ibis::bitvector \&bv, const std::vector $<$ uint32_t $>\&$ dim, std::vector $<$ uint32_t $>$ \&coords)

Convert positions in a bit vector to mesh coordinates.

\subsubsection{Detailed Description}

The class adds more functionality to ibis::query to handle data from meshes.

The new functions treats cells of meshes as connected regions in space.

\subsubsection{Member Function Documentation}

3.63.2.1 int ibis::meshQuery::bitvectorToCoordinates (const ibis::bitvector \& bv, const std::vector $<$ uint32_t $>\boldsymbol{\&} \operatorname{dim}, \mathbf{s t d}:$ :vector $<$ uint32_t $>\boldsymbol{\&}$ coords) $\quad$ [ s tat ic ]

Convert positions in a bit vector to mesh coordinates.

It converts the positions of bits that are 1 to coordinates in a regular mesh with deminsions given in dim. The C-sytle array ordering is assumed.

3.63.2.2 int ibis::meshQuery::getHitsAsRanges (std::vector $<$ std::vector $<$ uint32_t $>>\&$ reg, const bool merge $=$ false $)$ const

This variant of getHitsAsRanges uses the dimensions defined by ibis::table::getMeshShape().

\section{See also:}

ibis::meshQuery::getHitsAsRanges

ibis::table::getMeshShape

3.63.2.3 int ibis::meshQuery::getHitsAsRanges (std::vector $<$ std::vector $<$ uint32_t $>>\&$ reg, const std::vector $<$ uint32_t $>\&$ dim, const bool merge $=$ false) const

Translate hit vector into bounding boxes.

The bitmap is assumed to be a mapping of a regular grid with dimensions specified in variable dim. A row-major ordering (C style multiple dimensional arrays, NOT fortran style) is assumed, that is the slowest varying dimension is $\operatorname{dim}[0]$.

\section{Parameters:}

reg The return value that contains the list of ranges as hypercubes. Following the convention of typical $\mathrm{C} / \mathrm{C}++$ indexing scheme, the lower bounds of the ranges are inclusive and the upper bounds of the ranges are exclusive. For example, a 2D range $[2,3,5,10]$ covers points with coordinates $[2,5],[2,6],[2,7],[2,8]$ and $[2,9]$. This is an example of a line segment with five points. This function may generate hypercubes of any shape or size.

$\operatorname{dim}$ The size of the grid. The value dim.size() is the dimension of grid. Input argument, nor modified.

merge An optional argument. If true, will attempt to merge line segments generated to form larger hypercubes. Default to false because it make take a significant amount of time to merge the ranges.

This function returns an integer value with the following definition.

- 0 - conversion succeeded (no warning) 
- -1 - the bitvector length does not match the product of values in dim

- -2 - product of values in dim overflows an unsigned integer

- $-3-$ no hit vector to work with

- -4 - array dim is empty

Note:

It can only be called after the functions estimate or evaluate have been called. The ranges computed after calling estimate may be smaller than that computed after calling evaluate becaue estimate may not generate the exact answer to the query.

\subsubsection{4 int ibis::meshQuery::getPointsOnBoundary (std::vector $<$ std::vector $<$ uint32_t $>>\& b d y$ ) const}

The variant of getPointsOnBoundary use dimensions returned by ibis::table::getMeshShape().

\section{See also:}

ibis::meshQuery::getPointsOnBoundary

ibis::table::getMeshShape

3.63.2.5 int ibis::meshQuery::getPointsOnBoundary (std::vector $<$ std::vector $<$ uint32_t $>>\& b d y$, const std::vector $<$ uint32_t $>\&$ dim) const

Determine points with neighbors that are not hits.

\section{Parameters:}

bdy The return value that contains the list of points.

$\operatorname{dim}$ The size of the grid. The value dim.size() is the dimension of the grid. Each element of bdy is a std::vector with the same size as dim.

\section{See also:}

ibis::meshQuery::getHitsAsRanges

\section{Note:}

The inner array has the same number of elements as argument dim and the dimensions are ordered the same way as in dim as well. All functions in this class assumes that the mesh points are linearized using a raster scan order where dim[0] varies the slowest.

The documentation for this class was generated from the following files:

- meshQuery.h

- meshQuery.cpp

\subsection{4 ibis::moins Class Reference}

The multicomponent range code on bins.

\#include <ibin.h>

Inheritance diagram for ibis::moins:: 


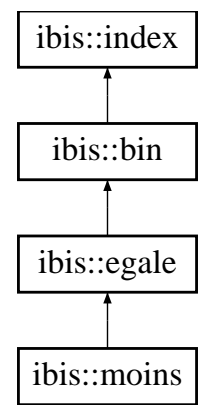

\section{Public Member Functions}

- long append (const array_t $<$ uint32_t $>$ \&ind)

Append a list of integers representing bin numbers.

- long append (const ibis::moins \&tail)

- virtual long append (const char $* \mathrm{dt}$, const char $* \mathrm{df}$, uint32_t nnew)

Extend the index.

- virtual uint32_t estimate (const ibis::qContinuousRange \&expr) const

Returns an upper bound on the number of hits.

- virtual void estimate (const ibis::qContinuousRange \&expr, ibis::bitvector \&lower, ibis::bitvector \&upper) const

Computes an approximation of hits as a pair of lower and upper bounds.

- virtual long evaluate (const ibis::qContinuousRange \&expr, ibis::bitvector \&hits) const

To evaluate the exact hits.

- virtual double getSum () const

Compute the approximate sum of all the values indexed.

- moins (const ibis::bin \&rhs, const uint32_t nbase=2)

- moins (const ibis::column $*$ c, ibis::fileManager::storage $*$ st, uint32_t offset=8)

- moins (const ibis::column $* \mathrm{c}=0$, const char $* \mathrm{f}=0$, const uint32_t nbase $=2$ )

- virtual const char $*$ name () const

Returns the name of the index, similar to the function type, but returns a string instead.

- virtual void print (std::ostream \&out) const

Prints human readable information.

- virtual void speedTest (std::ostream \&out) const

Time some logical operations and print out their speed.

- virtual INDEX_TYPE type () const

Returns an index type identifier.

- virtual void write (const char $* \mathrm{dt}$ ) const

Save index to a file. 


\section{Protected Member Functions}

- virtual double computeSum () const

\subsubsection{Detailed Description}

The multicomponent range code on bins.

Moins is a French word for 'less'.

\subsubsection{Member Function Documentation}

3.64.2.1 void ibis::moins::estimate (const ibis::qContinuousRange \& expr, ibis::bitvector \& lower, ibis::bitvector \& upper) const [virtual]

Computes an approximation of hits as a pair of lower and upper bounds.

\section{Parameters:}

expr the query expression to be evaluated.

lower a bitvector marking a subset of the hits. All rows marked with one (1) are definitely hits.

upper a bitvector marking a superset of the hits. All hits are marked with one, but some of the rows marked one may not be hits. If the variable upper is empty, the variable lower is assumed to contain the exact answer.

Reimplemented from ibis::egale.

3.64.2.2 long ibis::moins::evaluate (const ibis::qContinuousRange \& expr, ibis::bitvector \& hits) const [virtual]

To evaluate the exact hits.

On success, return the number of hits, otherwise a negative value is returned.

Reimplemented from ibis::egale.

\subsubsection{3 double ibis::moins::getSum () const [virtual]}

Compute the approximate sum of all the values indexed.

If it decides that computing the sum directly from the vertical partition is more efficient, it will return NaN immediately.

Reimplemented from ibis::egale.

\subsubsection{4 void ibis::moins::print (std::ostream \& out) const [virtual]}

Prints human readable information.

Outputs information about the index as text to the specified output stream.

Reimplemented from ibis::egale.

\subsubsection{5 void ibis::moins::write (const char $* \boldsymbol{d t}$ ) const [ virtual]}

Save index to a file.

Outputs the index in a compact binary format to the named file or directory. The index file contains a header that can be identified by the function isIndex.

Reimplemented from ibis::egale.

The documentation for this class was generated from the following files: 
- ibin.h

- icmoins.cpp

\subsection{5 ibis::nameList Class Reference}

A data structure to store a small set of names.

\#include <util.h>

\section{Public Types}

- typedef std::vector $<$ const char $*>$ ::const_iterator const_iterator

\section{Public Member Functions}

- const_iterator begin () const

- void clear ()

- bool empty () const

- const_iterator end () const

- size_t find (const char $*$ key) const

Find the order of the key in the list.

- nameList (const char $*$ str)

- const char $*$ operator $*()$ const

- const char $*$ operator[ ] (size_t i) const

- void select (const char $*$ str)

Add more names to the list.

- size_t size () const

\subsubsection{Detailed Description}

A data structure to store a small set of names.

The names are sorted in case-insensitive order.

\subsubsection{Member Function Documentation}

\subsubsection{1 size_t ibis::nameList::find (const char $*$ key) const}

Find the order of the key in the list.

If the key is in the list it returns the position of the key, otherwise it returns the size of the name list.

The documentation for this class was generated from the following files:

- util.h

- query.cpp 


\subsection{6 ibis::pack Class Reference}

A two-level index.

\#include <ibin.h>

Inheritance diagram for ibis::pack::

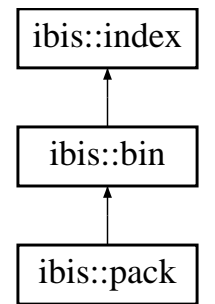

\section{Public Member Functions}

- virtual void adjustLength (uint32_t nrows)

- long append (const ibis::pack \&tail)

- virtual long append (const char $* \mathrm{dt}$, const char $* \mathrm{df}$, uint32_t nnew)

Extend the index.

- virtual void binBoundaries (std::vector $<$ double $>\&$ ) const

The function binBoundaries and binWeights return bin boundaries and counts of each bin respectively.

- virtual void binWeights (std::vector $<$ uint32_t $>\&$ ) const

- virtual void estimate (const ibis::qContinuousRange \&expr, ibis::bitvector \&lower, ibis::bitvector \&upper) const

Computes an approximation of hits as a pair of lower and upper bounds.

- virtual long evaluate (const ibis::qContinuousRange \&expr, ibis::bitvector \&hits) const

To evaluate the exact hits.

- virtual double getSum () const

Compute the approximate sum of all the values indexed.

- virtual const char $*$ name () const

Returns the name of the index, similar to the function type, but returns a string instead.

- virtual uint32_t numBins () const

- pack (const ibis::bin \&rhs)

- pack (const ibis::column $*$ c, ibis::fileManager::storage $*$ st, uint32_t offset=8)

- virtual void print (std::ostream \&out) const

Prints human readable information.

- virtual void read (ibis::fileManager::storage $*$ st)

Reconstructs an index from an array of bytes.

- virtual void read (const char $*$ idxfile)

Reconstructs an index from the named file.

- virtual void speedTest (std::ostream \&out) const

Time some logical operations and print out their speed. 
- virtual INDEX_TYPE type () const

Returns an index type identifier.

- virtual float undecidable (const ibis::qContinuousRange \&expr, ibis::bitvector \&iffy) const

Mark the position of the rows that can not be decided with this index.

- virtual void write (const char $* \mathrm{dt})$ const

Save index to a file.

\section{Protected Member Functions}

- virtual double computeSum () const

The the approximate sum of all values using the top level bins.

\subsubsection{Detailed Description}

A two-level index.

Coarse level is cumulative, but not the bottom level.

\subsubsection{Member Function Documentation}

3.66.2.1 void ibis::pack::estimate (const ibis::qContinuousRange \& expr, ibis::bitvector \& lower, ibis::bitvector \& upper) const [virtual]

Computes an approximation of hits as a pair of lower and upper bounds.

\section{Parameters:}

expr the query expression to be evaluated.

lower a bitvector marking a subset of the hits. All rows marked with one (1) are definitely hits.

upper a bitvector marking a superset of the hits. All hits are marked with one, but some of the rows marked one may not be hits. If the variable upper is empty, the variable lower is assumed to contain the exact answer.

Reimplemented from ibis::bin.

3.66.2.2 long ibis::pack::evaluate (const ibis::qContinuousRange \& expr, ibis::bitvector \& hits) const [virtual]

To evaluate the exact hits.

On success, return the number of hits, otherwise a negative value is returned.

Reimplemented from ibis::bin.

\subsubsection{3 double ibis::pack::getSum () const [virtual]}

Compute the approximate sum of all the values indexed.

If it decides that computing the sum directly from the vertical partition is more efficient, it will return NaN immediately.

Reimplemented from ibis::bin. 


\subsubsection{4 void ibis::pack::print (std::ostream \& out) const [virtual]}

Prints human readable information.

Outputs information about the index as text to the specified output stream.

Reimplemented from ibis::bin.

\subsubsection{5 void ibis::pack::read (ibis::fileManager::storage $* s t$ ) [virtual]}

Reconstructs an index from an array of bytes.

Intended for internal use only!

Reimplemented from ibis::bin.

\subsubsection{6 void ibis::pack::read (const char $*$ idxfile) $\quad$ [virtual]}

Reconstructs an index from the named file.

The name can be the directory containing an index file. In this case, the name of the index file must be the name of the column followed by ".idx" suffix.

Reimplemented from ibis::bin.

3.66.2.7 float ibis::pack::undecidable (const ibis::qContinuousRange \& expr, ibis::bitvector \& iffy) const [virtual]

Mark the position of the rows that can not be decided with this index.

\section{Parameters:}

expr the range conditions to be evaluated.

iffy the bitvector marking the positions of rows that can not be decided using the index. Return value is the expected fraction of undecided rows that might satisfy the range conditions.

Reimplemented from ibis::bin.

\subsubsection{8 void ibis::pack::write (const char $* \boldsymbol{d t}$ ) const [virtual]}

Save index to a file.

Outputs the index in a compact binary format to the named file or directory. The index file contains a header that can be identified by the function isIndex.

Reimplemented from ibis::bin.

The documentation for this class was generated from the following files:

- ibin.h

- ixpack.cpp

\subsection{7 ibis::pale Class Reference}

A two-level index.

\#include <ibin.h>

Inheritance diagram for ibis::pale:: 


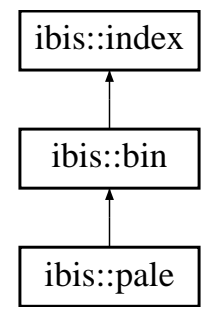

\section{Public Member Functions}

- virtual void adjustLength (uint32_t nrows)

- long append (const ibis::pale \&tail)

- virtual long append (const char $* \mathrm{dt}$, const char $* \mathrm{df}$, uint32_t nnew)

Extend the index.

- virtual void binBoundaries (std::vector $<$ double $>\&$ ) const

The function binBoundaries and binWeights return bin boundaries and counts of each bin respectively.

- virtual void binWeights (std::vector $<$ uint32_t $>$ \&) const

- virtual void estimate (const ibis::qContinuousRange \&expr, ibis::bitvector \&lower, ibis::bitvector \&upper) const

Computes an approximation of hits as a pair of lower and upper bounds.

- virtual long evaluate (const ibis::qContinuousRange \&expr, ibis::bitvector \&hits) const

To evaluate the exact hits.

- virtual const char $*$ name () const

Returns the name of the index, similar to the function type, but returns a string instead.

- virtual uint32_t numBins () const

- pale (const ibis::bin \&rhs)

- pale (const ibis::column $*$ c, ibis::fileManager::storage $*$ st, uint32_t offset=8)

- virtual void print (std::ostream \&out) const

Prints human readable information.

- virtual void read (ibis::fileManager::storage $*$ st)

Reconstructs an index from an array of bytes.

- virtual void read (const char $*$ idxfile)

Reconstructs an index from the named file.

- virtual void speedTest (std::ostream \&out) const

Time some logical operations and print out their speed.

- virtual INDEX_TYPE type () const

Returns an index type identifier.

- virtual float undecidable (const ibis::qContinuousRange \&expr, ibis::bitvector \&iffy) const

Mark the position of the rows that can not be decided with this index.

- virtual void write (const char $* \mathrm{dt}$ ) const

Save index to a file. 


\subsubsection{Detailed Description}

A two-level index.

Coarse level not cumulative, fine level is cumulative.

\subsubsection{Member Function Documentation}

3.67.2.1 void ibis::pale::estimate (const ibis::qContinuousRange \& expr, ibis::bitvector \& lower, ibis::bitvector \& upper) const [virtual]

Computes an approximation of hits as a pair of lower and upper bounds.

\section{Parameters:}

expr the query expression to be evaluated.

lower a bitvector marking a subset of the hits. All rows marked with one (1) are definitely hits.

upper a bitvector marking a superset of the hits. All hits are marked with one, but some of the rows marked one may not be hits. If the variable upper is empty, the variable lower is assumed to contain the exact answer.

Reimplemented from ibis::bin.

3.67.2.2 long ibis::pale::evaluate (const ibis::qContinuousRange \& expr, ibis::bitvector \& hits) const [virtual]

To evaluate the exact hits.

On success, return the number of hits, otherwise a negative value is returned.

Reimplemented from ibis::bin.

\subsubsection{3 void ibis::pale::print (std::ostream \& out) const [virtual]}

Prints human readable information.

Outputs information about the index as text to the specified output stream.

Reimplemented from ibis::bin.

3.67.2.4 void ibis::pale::read (ibis::fileManager::storage $*$ st) [virtual]

Reconstructs an index from an array of bytes.

Intended for internal use only!

Reimplemented from ibis::bin.

\subsubsection{5 void ibis::pale::read (const char $*$ idxfile) [virtual]}

Reconstructs an index from the named file.

The name can be the directory containing an index file. In this case, the name of the index file must be the name of the column followed by ".idx" suffix.

Reimplemented from ibis::bin.

3.67.2.6 float ibis::pale::undecidable (const ibis::qContinuousRange \& expr, ibis::bitvector \& iffy) const [virtual]

Mark the position of the rows that can not be decided with this index. 


\section{Parameters:}

expr the range conditions to be evaluated.

iffy the bitvector marking the positions of rows that can not be decided using the index. Return value is the expected fraction of undecided rows that might satisfy the range conditions.

Reimplemented from ibis::bin.

\subsubsection{7 void ibis::pale::write (const char $* \boldsymbol{d t})$ const $\quad$ [ virtual]}

Save index to a file.

Outputs the index in a compact binary format to the named file or directory. The index file contains a header that can be identified by the function isIndex.

Reimplemented from ibis::bin.

The documentation for this class was generated from the following files:

- ibin.h

- ixpale.cpp

\subsection{8 ibis::part Class Reference}

The class ibis::part represents a partition of a relational table.

\#include <part.h>

\section{Public Types}

- typedef std::map $<$ const char $*$, column $*$, lessi $>$ columnList

- enum TABLE_STATE \{

UNKNOWN_STATE $=0$, STABLE_STATE, RECEIVING_STATE, PRETRANSITION_STATE, TRANSITION_STATE, POSTTRANSITION_STATE \}

\section{Public Member Functions}

- ibis::fileManager::ACCESS_PREFERENCE accessHint (const ibis::bitvector \&mask, unsigned elemsize=4) const

Evaluate the strategy to access a data file.

- long append (const char $*$ dir)

Append data in dir to the current database.

- void buildIndex (int nthr=1)

Load and immediately unload indices.

- void combineNames (ibis::table::namesTypes \&metalist) const

Update the list of columns with information in this data partition.

- long commit (const char $*$ dir)

Commit the active database.

- void computeMinMax ()

Go through all the values to compute the min and max for each column. 
- long countHits (const ibis::qRange \&cmp) const

Count the number of hits for a single range condition.

- const char $*$ currentDataDir () const

Return the name of the active data directory.

- const char $*$ description () const

Return a text description of the partition.

- void doBackup ()

A function to start backing up the active dir.

- template $<$ typename E $>$ long doScan (const array_t $<$ E $>$ \&varr, const ibis::qContinuousRange \&cmp, const ibis::bitvector \&mask, ibis::bitvector \&hits) const

- template $<$ typename $\mathrm{E}>$ long doScan (const array_t $<\mathrm{E}>$ \&varr, const ibis::qRange \&cmp, const ibis::bitvector \&mask, ibis::bitvector \&hits) const

Locate the records that satisfy the range condition.

- virtual long doScan (const ibis::compRange \&cmp, const ibis::bitvector \&mask, ibis::bitvector \&hits, ibis::compRange::barrel $*$ bar $=0$ ) const

Locate the records that have mark value 1 and satisfy the complex range conditions.

- virtual long doScan (const ibis::compRange \&cmp, ibis::bitvector \&hits) const

Locate the records that satisfy the complex range condition.

- virtual long doScan (const ibis::qRange \&cmp, const ibis::bitvector \&mask, ibis::bitvector \&hits) const

Evalute the range condition on the records that are marked 1 in the mask.

- virtual long doScan (const ibis::qRange \&cmp, ibis::bitvector \&hits) const

Evaluate the range condition.

- double estimateCost (const ibis::qMultiString \&cmp) const

- double estimateCost (const ibis::qString \&cmp) const

- double estimateCost (const ibis::qDiscreteRange \&cmp) const

- double estimateCost (const ibis::qContinuousRange \&cmp) const

Estimate the cost of evaluate the query expression.

- virtual long estimateMatchAny (const ibis::qAnyAny \&cmp, ibis::bitvector \&low, ibis::bitvector \&high) const Estimate a lower bound and an upper bound on the records that are hits.

- long estimateRange (const ibis::qDiscreteRange \&cmp, ibis::bitvector \&low, ibis::bitvector \&high) const

Estimate the discrete range condition.

- long estimateRange (const ibis::qContinuousRange \&cmp, ibis::bitvector \&low, ibis::bitvector \&high) const

- long estimateRange (const ibis::qDiscreteRange \&cmp) const

Return an upper bound on the number of hits.

- long estimateRange (const ibis::qContinuousRange \&cmp) const

Return an upper bound on the number of hits.

- int64_t evaluateJoin (const std::vector $<$ const ibis::rangeJoin $*>$ \&cmp, const ibis::bitvector64 \&trial, ibis::bitvector64 \&result) const 
Check a set of pairs defined in trial.

- int64_t evaluateJoin (const ibis::rangeJoin \&cmp, const ibis::bitvector64 \&trial, ibis::bitvector64 \&result) const

Evaluate all pairs in trial to determine whether they really satisfy the range join defined in $\mathrm{cmp}$.

- int64_t evaluateJoin (const std::vector $<$ const ibis::rangeJoin $*>$ \&cmp, const ibis::bitvector \&mask) const

- int64_t evaluateJoin (const std::vector $<$ const ibis::rangeJoin $*>\& \mathrm{cmp}$, const ibis::bitvector \&mask, ibis::bitvector64 \&pairs) const

Evaluate a join defined with multiple (conjunctive) range join conditions.

- int64_t evaluateJoin (const ibis::rangeJoin \&cmp, const ibis::bitvector \&mask) const

Return only the number of pairs satisfying the join condition.

- int64_t evaluateJoin (const ibis::rangeJoin \&cmp, const ibis::bitvector \&mask, const char *pairfile) const

Return the number of pairs satisfying the join condition.

- int64_t evaluateJoin (const ibis::rangeJoin \&cmp, const ibis::bitvector \&mask, ibis::bitvector64 \&pairs) const

Evaluate a self-join.

- long evaluateRange (const ibis::qDiscreteRange \&cmp, const ibis::bitvector \&mask, ibis::bitvector \&res) const

- long evaluateRange (const ibis::qContinuousRange \&cmp, const ibis::bitvector \&mask, ibis::bitvector \&res) const

- long evaluateRIDSet (const ibis::RIDSet \&, ibis::bitvector \&) const

- long get1DDistribution (const char $*$ constraints, const char $*$ cname, double begin, double end, double stride, std::vector $<$ size_t $>$ \& counts) const

Count the number of records falling in the bins defined by the begin:end:stride triplets.

- long get2DDistribution (const char $*$ constraints, const char $*$ cname1, double begin1, double end1, double stride1, const char $*$ cname 2 , double begin 2 , double end2, double stride2, std::vector $<$ size_t $>\&$ counts) const

ibis::part::get1DDistribution ibis::table::getHistogram2D

- long get3DDistribution (const char $*$ constraints, const char $*$ cname1, double begin1, double end1, double stride1, const char $*$ cname 2 , double begin 2 , double end2, double stride2, const char $*$ cname 3 , double begin 3 , double end3, double stride3, std::vector $<$ size_t $>$ \& counts) const

ibis::part::get1DDistribution ibis::table::getHistogram $3 D$

- double getActualMax (const char $*$ name) const

The actual maximum value in the named column.

- double getActualMin (const char *name) const

The actual minimum value in the named column.

- column $*$ getColumn (uint32_t ind) const

Returns the pointer to the ith column.

- column $*$ getColumn (const char $*$ name) const

Given a name, return the associated column.

- double getColumnSum (const char *name) const

Sum of all value in the named column. 
- long getCumulativeDistribution (const char $*$ constraints, const char $*$ name, uint32_t nbc, double $*$ bounds, uint32_t $*$ counts) const

Compute the conditional distribution and return the distribution in the arrays provided.

- long getCumulativeDistribution (const char $*$ name, uint32_t nbc, double $*$ bounds, uint32_t $*$ counts) const

This version of get CumulativeDistribution uses two user supplied arrays bounds and counts.

- long getCumulativeDistribution (const char $*$ constraints, const char $*$ name, std::vector $<$ double $>\&$ bounds, std::vector $<$ uint32_t $>\&$ counts) const

Compute the cumulative distribution of the variable named name under the specified constraints.

- long getCumulativeDistribution (const char $*$ name, std::vector $<$ double $>$ \&bounds, std::vector $<$ uint32_t $>$ \&counts) const

Compute a cumulative distribution (a cumulative histogram).

- long getDistribution (const char $*$ name, const char $*$ constraints, uint32_t nbc, double $*$ bounds, uint32_$\mathrm{t} *$ counts) const

Compute the conditional binned data distribution with the specified maximum number of bins.

- long getDistribution (const char $*$ name, uint32_t nbc, double $*$ bounds, uint32_t $*$ counts) const

Compute the binned distribution with the specified maximum number of bins.

- long getDistribution (const char $*$ constraints, const char $*$ name, std::vector $<$ double $>$ \&bounds, std::vector $<$ uint32_t $>$ \&counts) const

Compute the conditional binned data distribution.

- long getDistribution (const char $*$ name, std::vector $<$ double $>$ \&bounds, std::vector $<$ uint32_t $>\&$ counts) const

Compute the binned distribution of the name variable.

- info $*$ getInfo () const

Return descriptive information about the data partition.

- long getJointDistribution (const char $*$ constraints, const char $*$ name1, const char $*$ name2, std::vector $<$ double $>$ \&bounds1, std::vector $<$ double $>$ \&bounds2, std::vector $<$ uint32_t $>$ \&counts) const

Compute the joint distribution of two variables.

- const std::vector $<$ std::string $>\&$ getMeshDimensions () const

Return the name of the dimensions corresponding to the vector returned from getMeshShape.

- const std::vector $<$ uint32_t $>$ \& getMeshShape () const

In many scientific applications, data are defined on meshes.

- const char $*$ getMetaTag (const char $*$ ) const

Return the value of the meta tag with the specified name.

- array_t $<$ rid_t $>*$ getRIDs (const ibis::bitvector \&mask) const

- $\operatorname{array} \_\mathrm{t}<$ rid_t $>*$ getRIDs () const

- uint32_t getRowNumber (const rid_t \&rid) const

Return the row number of the row with specified RID.

- TABLE_STATE getState () const

Return the current state of data partition. 
- TABLE_STATE getStateNoLocking () const

- float getUndecidable (const ibis::qDiscreteRange \&cmp, ibis::bitvector \&iffy) const

Discover the records that can not be decided using the index.

- float getUndecidable (const ibis::qContinuousRange \&cmp, ibis::bitvector \&iffy) const

Discover the records that can not be decided using the index.

- bool hasRIDs () const

- void indexSpec (const char $*$ )

Replace existing index specification with a new one.

- const char $*$ indexSpec () const

Return the current index specification.

- void loadIndex (const char $*$ opt $=0$ ) const

Load all indices.

- void logMessage (const char $*$ event, const char $* \mathrm{fmt}, \ldots$ ) const

- void logWarning (const char $*$ event, const char $* \mathrm{fmt}, \ldots$ ) const

- long lookforString (const ibis::qMultiString \& cmp) const

- long lookforString (const ibis::qString \&cmp) const

Return an upper bound of the number of records that have the exact string value.

- long lookforString (const ibis::qMultiString \&cmp, ibis::bitvector \&low) const

Determine the records that have the exact string values.

- long lookforString (const ibis::qString \&cmp, ibis::bitvector \&low) const

Find all records that has the exact string value.

- virtual long matchAny (const ibis::qAnyAny \&cmp, const ibis::bitvector \&mask, ibis::bitvector \&hits) const

Perform exact match operation for an AnyAny query.

- virtual long matchAny (const ibis::qAnyAny \&cmp, ibis::bitvector \&hits) const

- bool matchMetaTags (const ibis::resource::vList \&mtags) const

Match multiple name-value pairs.

- bool matchMetaTags (const std::vector $<$ const char $*>$ \&mtags) const

Match multiple name-value pairs against the internally stored meta tags.

- bool matchNameValuePair (const char *name, const char *value) const

Match a name-value pair in the meta tags.

- std::string metaTags () const

Return the list of meta tags as a single string.

- const char * name () const

Return the name of the partition.

- size_t nColumns () const

Return the number of attributes in the partition.

- virtual long negativeScan (const ibis::qRange \&cmp, const ibis::bitvector \&mask, ibis::bitvector \&hits) const 
Compute the records (marked 1 in the mask) that does not satisfy the range condition.

- size_t nRows () const

Return the number of rows.

- part (const ibis::resource::vList \&mtags)

- part (const std::vector $<$ const char $*>$ \&mtags)

- part (const char $*$ adir, const char $*$ bdir)

Initialize a table from the named directories.

- part (const char $*$ prefix $=0$ )

Initialize a table object. Use gParameters to get the file names.

- void print (std::ostream \&out) const

Output a description of every column of the data partition.

- void purgeIndexFiles () const

Remove existing index files!

- void queryTest (const char $*$ pref, long $*$ nerrors) const

- void quickTest (const char $*$ pref, long $*$ nerrors) const

- long reorder (const ibis::table::stringList \&names)

- long reorder ()

Reorder all columns of a partition.

- long rollback ()

Rollback(revert) to previous data set.

- array_t $<$ double $>*$ selectDoubles (const char $*$ name, const ibis::bitvector \&mask) const

- array_t $<$ float $>*$ selectFloats (const char $*$ name, const ibis::bitvector \&mask) const

- array_t $<$ int32_t $>*$ selectInts (const char $*$ name, const ibis::bitvector \&mask) const

- array_t $<$ int64_t $>*$ selectLongs (const char $*$ name, const ibis::bitvector \&mask) const

- array_t $<$ uint32_t $>*$ selectUInts (const char $*$ name, const ibis::bitvector \&mask) const

- virtual long selfTest (int nth $=1$, const char $*$ pref $=0$ ) const

Perform predefined set of tests and return the number of failures.

- void setMeshShape (const char $*$ shape)

Digest the mesh shape stored in the string.

- time_t timestamp () const

Return the time stamp on the partition.

- void unloadIndex () const

Unload index.

- void updateTDC () const

Write the TDC file to record the changes to the partition. 


\section{Static Public Member Functions}

- static uint32_t countPages (const ibis::bitvector \&mask, unsigned elemsize=4)

Given a bitvector, compute the number of pages would be accessed.

- static void genName (const ibis::resource::vList \&mtags, std::string \&name)

Generate name for a partition based on the meta tags.

- static void genName (const std::vector $<$ const char $*>$ \&mtags, std::string \&name)

Generate name for a partition based on the meta tags.

- static char $*$ readMetaTags (const char $*$ const dir)

A class function to read the meta tags in the tdc file.

\section{Protected Member Functions}

- long append1 (const char $*$ dir)

- long append2 (const char $*$ dir)

- long appendToBackup (const char $*$ dir)

Append data in dir to the partition in the backup directory.

- template $<$ typename T1, typename T2 $>$ long count2DBins (const array_t $<\mathrm{T} 1>\&$ vals1, const double \&begin1, const double \&end1, const double \&stride1, const array_t $<\mathrm{T} 2>$ \&vals2, const double \&begin2, const double \&end2, const double \&stride2, std::vector $<$ size_t $>$ \& counts) const

Count the number of values falling in $2 D$ bins.

- template $<$ typename T1, typename T2, typename T3 $>$ long count3DBins (const array_t $<\mathrm{T} 1>$ \&vals1, const double \&begin1, const double \&end1, const double \&stride1, const array_t $<\mathrm{T} 2>$ \&vals2, const double \&begin2, const double \&end2, const double \&stride2, const array_t $<\mathrm{T} 3>$ \&vals3, const double \&begin3, const double \&end3, const double \&stride3, std::vector $<$ size_t $>$ \&counts) const

Count the number of values falling in $3 D$ bins.

- void deriveBackupDirName ()

- void digestMeshShape (const char $*$ shape)

Convert the string describing the shape into internal storage format.

- void doByteCompare (const char $*$ file, const ibis::bitvector \&mask, ibis::bitvector \&hits, const ibis::qRange \&cmp) const

- template $<$ typename T, typename F1, typename F2 $>$ long doCompare (const array_t $<\mathrm{T}>$ \& vals, F1 cmp1, F2 cmp2, const ibis::bitvector \&mask, ibis::bitvector \&hits) const

The actual scan function.

- template $<$ typename $\mathrm{T}$, typename $\mathrm{F}>$ long doCompare (const array_t $<\mathrm{T}>$ \&vals, F cmp, const ibis::bitvector \&mask, ibis::bitvector \&hits) const

Accepts an externally passed comparison operator.

- template $<$ typename $\mathrm{T}>$ void doCompare (const char $*$ file, const ibis::bitvector \&mask, ibis::bitvector \&hits, const ibis::qRange \&cmp) const

- template $<$ typename $\mathrm{T}>$ void doCompare (const array_t $<\mathrm{T}>$ \&array, const ibis::bitvector \&mask, ibis::bitvector \&hits, const ibis::qRange \&cmp) const

- void doCompare (const array_t $<$ double $>$ \&array, const ibis::bitvector \&mask, ibis::bitvector \&hits, const ibis::qRange \&cmp) const 
- void doCompare (const array_t $<$ float $>$ \&array, const ibis::bitvector \&mask, ibis::bitvector \&hits, const ibis::qRange \&cmp) const

- void doCompare (const array_t $<$ uint32_t $>$ \&array, const ibis::bitvector \&mask, ibis::bitvector \&hits, const ibis::qRange \&cmp) const

- void doCompare (const array_t $<$ int32_t $>$ \&array, const ibis::bitvector \&mask, ibis::bitvector \&hits, const ibis::qRange \&cmp) const

- template $<$ typename $\mathrm{T}$, typename $\mathrm{F} 1$, typename F2 $>$ long doCompare0 (const array_t $<\mathrm{T}>$ \& vals, $\mathrm{F} 1 \mathrm{cmp} 1$, F2 cmp2, const ibis::bitvector \&mask, ibis::bitvector \&hits) const

This version uses uncompressed bitvector to store the scan results internally.

- template $<$ typename T, typename $\mathrm{F}>$ long doCompare0 (const array_t $<\mathrm{T}>$ \&vals, $\mathrm{F}$ cmp, const ibis::bitvector \&mask, ibis::bitvector \&hits) const

This version uses an uncompressed bitvector to store the scan results internally.

- template $<$ typename $\mathrm{T}$, typename $\mathrm{F} 1$, typename $\mathrm{F} 2>$ long doCount (const array_t $<\mathrm{T}>$ \&vals, const ibis::bitvector \&mask, F1 cmp1, F2 cmp2) const

- template $<$ typename $\mathrm{T}$, typename $\mathrm{F}>$ long doCount (const array_t $<\mathrm{T}>$ \&vals, const ibis::bitvector \&mask, $\mathrm{F}$ cmp) const

- template $<$ typename $\mathrm{T}>$ long doCount (const array_t $<\mathrm{T}>$ \&vals, const ibis::qRange \&cmp, const ibis::bitvector \&mask) const

- template $<$ typename $\mathrm{T}>$ long doCount (const ibis::qRange \&cmp) const

- void doDoubleCompare (const char $*$ file, const ibis::bitvector \&mask, ibis::bitvector \&hits, const ibis::qRange \&cmp) const

- void doFloatCompare (const char $*$ file, const ibis::bitvector \&mask, ibis::bitvector \&hits, const ibis::qRange \&cmp) const

- void doIntCompare (const char $*$ file, const ibis::bitvector \&mask, ibis::bitvector \&hits, const ibis::qRange \&cmp) const

- void doShortCompare (const char $*$ file, const ibis::bitvector \&mask, ibis::bitvector \&hits, const ibis::qRange \&cmp) const

- void doUByteCompare (const char $*$ file, const ibis::bitvector \&mask, ibis::bitvector \&hits, const ibis::qRange \&cmp) const

- void doUIntCompare (const char $*$ file, const ibis::bitvector \&mask, ibis::bitvector \&hits, const ibis::qRange \&cmp) const

- void doUShortCompare (const char $*$ file, const ibis::bitvector \&mask, ibis::bitvector \&hits, const ibis::qRange \&cmp) const

- void extendMetaTags ()

- void freeRIDs () const

Remove the rids list from memory.

- void logError (const char $*$ event, const char $* \mathrm{fmt}, \ldots$ ) const

Log functions.

- void makeBackupCopy ()

- template $<$ typename $\mathrm{T}>$ void negativeCompare (const char $*$ file, const ibis::bitvector \&mask, ibis::bitvector \&hits, const ibis::qRange \&cmp) const

- template $<$ typename $\mathrm{T}>$ void negativeCompare (const array_t $<\mathrm{T}>$ \&array, const ibis::bitvector \&mask, ibis::bitvector \&hits, const ibis::qRange \&cmp) const

- void negativeCompare (const array_t $\mathrm{t}$ double $>$ \&array, const ibis::bitvector \&mask, ibis::bitvector \&hits, const ibis::qRange \&cmp) const

- void negativeCompare (const array_t $<$ float $>$ \&array, const ibis::bitvector \&mask, ibis::bitvector \&hits, const ibis::qRange \&cmp) const

- void negativeCompare (const array_t $<$ uint32_t $>$ \&array, const ibis::bitvector \&mask, ibis::bitvector \&hits, const ibis::qRange \&cmp) const

- void negativeCompare (const array_t $<$ int32_t $>$ \&array, const ibis::bitvector \&mask, ibis::bitvector \&hits, const ibis::qRange \&cmp) const 
- void negativeDoubleCompare (const char $*$ file, const ibis::bitvector \&mask, ibis::bitvector \&hits, const ibis::qRange \&cmp) const

- void negativeFloatCompare (const char $*$ file, const ibis::bitvector \&mask, ibis::bitvector \&hits, const ibis::qRange \&cmp) const

- void negativeIntCompare (const char $*$ file, const ibis::bitvector \&mask, ibis::bitvector \&hits, const ibis::qRange \&cmp) const

- void negativeUIntCompare (const char $*$ file, const ibis::bitvector \&mask, ibis::bitvector \&hits, const ibis::qRange \&cmp) const

- long packCumulativeDistribution (const std::vector $<$ double $>$ \&bounds, const std::vector $<$ uint32_t $>$ \&counts, uint32_t nbc, double $*$ bptr, uint32_t $*$ cptr) const

Pack a cumulative distribution stored in two std::vectors into two arrays provided by the caller.

- long packDistribution (const std::vector $<$ double $>$ \&bounds, const std::vector $<$ uint32_t $>$ \&counts, uint32_t nbc, double $*$ bptr, uint32_t $*$ cptr) const

Pack a binned distribution.

- void readMeshShape (const char $*$ const dir)

Read shape of the mesh from tdc file.

- void readRIDs () const

Read RIDs from file 'rids'.

- int readTDC (size_t \&nrows, columnList \&plist, const char $*$ dir)

Read TDC file.

- template $<$ typename $\mathrm{T}>$ long reorderValues (const char $*$ fname, const array_t $\mathrm{t}<$ uint32_t $>$ \&indin, array_t $<$ uint32_t $>$ \&indout, array_t $<$ uint32_t $>$ \&starts)

Write the named data file in a segmented sorted order.

- void setMetaTags (const std::vector $<$ const char $*>$ \&mts)

Make a deep copy of the incoming name-value pairs.

- void setMetaTags (const ibis::resource::vList \&mts)

Make a deep copy of the incoming name-value pairs.

- long verifyBackupDir ()

- void writeTDC (const uint32_t nrows, const columnList \&plist, const char $*$ dir) const

Write TDC file.

- template $<$ typename $\mathrm{T}>$ long writeValues (const char $*$ fname, const array_t $<$ uint32_t $>$ \&ind)

Write the named data file with values in the given order.

\section{Protected Attributes}

- char $*$ activeDir

The active data directory.

- $\operatorname{char} *$ backupDir

The backup data directory.

- std::vector $<$ const column $*>$ colorder

An ordering of columns. 
- columnList columns

List of the columns.

- char $*$ idxstr

Index specification.

- char $*$ m_desc

Free form description of the partition.

- char $*$ m_name

Name of the data partition.

- ibis::resource::vList metaList

Meta tags as name-value pairs.

- ibis::part::cleaner $*$ myCleaner

The cleaner for the file manager.

- size_t nEvents

Number of events (rows) in the partition.

- array_t $\mathrm{t}<$ rid_t $>*$ rids

The object IDs (row id).

- std::vector $<$ std::string > shapeName

Names of the dimensions.

- std::vector $<$ uint32_t $>$ shapeSize

Sizes of the dimensions.

- TABLE_STATE state

- time_t switchTime

Time of last switch operation.

\section{Friends}

- class advisoryLock

- class cleaner

- struct info

- class mutexLock

- class readLock

- class writeLock

\section{Classes}

- class advisoryLock

An non-blocking version of writeLock.

- class barrel

To read a list of variables at the same time. 
- class cleaner

A cleaner to be used by the fileManager:: unload function.

- struct indexBuilderPool

- struct info

A simple class to describe an ibis::part object.

- class mutexLock

Provide a mutual exclusion lock on an ibis::part object.

- class readLock

Provide a read lock on an ibis::part.

- struct thrArg

- class vault

To read variables in certain order.

- class writeLock

Provide a write lock on an ibis::part.

\subsubsection{Detailed Description}

The class ibis::part represents a partition of a relational table.

The current implementation is designed to work with vertically partitioned data files. This class contains common information and operations on a partition.

\subsubsection{Constructor \& Destructor Documentation}

3.68.2.1 ibis::part::part (const char $*$ adir, const char $*$ bdir)

Initialize a table from the named directories.

Must have full and complete path.

\subsubsection{Member Function Documentation}

\subsubsection{1 long ibis::part::append (const char $*$ dir)}

Append data in dir to the current database.

Return the number of rows actually added. It is possible to rollback the append operation before commit.

\subsubsection{2 long ibis::part::appendToBackup (const char $*$ dir) $\quad$ [protected]}

Append data in dir to the partition in the backup directory.

Return the number of rows actually appended.

\subsubsection{3 long ibis::part::commit (const char $*$ dir)}

Commit the active database.

No longer able to rollback afterward. Return the number of records committed. 
3.68.3.4 template $<$ typename T, typename F1, typename F2 $>$ long ibis::part::doCompare (const array_t $<$ T $>\&$ vals, F1 cmp1, F2 cmp2, const ibis::bitvector \& mask, ibis::bitvector \& hits) const [protected]

The actual scan function.

This one chooses whether the internal bitvector for storing the scan results will be compressed or not. It always returns a compressed bitvector.

3.68.3.5 template $<$ typename $T$, typename $F>$ long ibis::part::doCompare (const array_t $t<T<\&$ vals, $F$ cmp, const ibis::bitvector \& mask, ibis::bitvector \& hits) const [protected]

Accepts an externally passed comparison operator.

It chooses whether the bitvector hits will be compressed internally based on the number of set bits in the mask.

3.68.3.6 template $<$ typename $\mathbf{E}>$ long ibis::part::doScan (const array_t $<\mathbf{E}>\boldsymbol{\&}$ varr, const ibis::qRange $\&$ cmp, const ibis: :bitvector \& mask, ibis::bitvector \& hits) const

Locate the records that satisfy the range condition.

Since the values are provided, this function does not check the name of the variable involved in the range condition.

3.68.3.7 long ibis::part::doScan (const ibis::qRange \& cmp, ibis::bitvector \& hits) const [virtual ]

Evaluate the range condition.

Scan the base data to resolve the range condition.

3.68.3.8 long ibis::part::estimateMatchAny (const ibis::qAnyAny \& cmp, ibis::bitvector \& low, ibis::bitvector \& high) const [virtual]

Estimate a lower bound and an upper bound on the records that are hits.

The bitvector low contains records that are hits (for sure) and the bitvector high contains records that are possible hits.

3.68.3.9 int64_t ibis::part::evaluateJoin (const std::vector $<$ const ibis::rangeJoin $*>\&$ cmp, const ibis::bitvector64 \& trial, ibis::bitvector64 \& result) const

Check a set of pairs defined in trial.

This version works on multiple (conjunctive) join conditions.

3.68.3.10 int64_t ibis::part::evaluateJoin (const ibis::rangeJoin \& cmp, const ibis::bitvector64 \& trial, ibis: :bitvector64 \& result) const

Evaluate all pairs in trial to determine whether they really satisfy the range join defined in cmp.

The result is stored in the argument result. This function returns the number of hits found.

3.68.3.11 int64_t ibis::part::evaluateJoin (const ibis::rangeJoin \& cmp, const ibis::bitvector \& mask, const char $*$ pairfile) const [inline ]

Return the number of pairs satisfying the join condition.

In addition, write the pairs into the file named pairfile.

3.68.3.12 int64_t ibis::part::evaluateJoin (const ibis::rangeJoin \& cmp, const ibis::bitvector \& mask, ibis::bitvector64 \& pairs) const [inline]

Evaluate a self-join. 
Return the number of pairs satisfying join condition. Only records marked with mask $=1$ are considered. The result pairs are stored in the bitvector pairs. A pair $<\mathrm{i}, \mathrm{j}>$ would be marked at position $\mathrm{i} * \mathrm{nRows}()+\mathrm{j}$ in pairs.

3.68.3.13 long ibis::part::get1DDistribution (const char $*$ constraints, const char $*$ cname, double begin, double end, double stride, std::vector $<$ size_t $>\&$ counts) const

Count the number of records falling in the bins defined by the begin:end:stride triplets.

The triplets defines

std: : ceil ( (end-begin)/stride)

bins. When this function completes successfully, it the array counts should

std: : ceil( (end-begin)/stride)

values, one for each bin. The return value should be the number of bins, otherwise, it indicates an error. If array counts has the same size as the number of bins on input, the count values will be added to the array. This is intended to be used to accumulate counts from different data partitions. If the array counts does not have the correct size, it will be resized to the correct size and initialized to contain only zero before counting the the current data partition.

ibis::table::getHistogram

\subsubsection{4 ibis::column $*$ ibis::part::getColumn (const char $*$ name) const $\quad$ [ in line ]}

Given a name, return the associated column.

Return nil pointer if the name is not found.

3.68.3.15 long ibis::part::getCumulativeDistribution (const char $*$ constraints, const char $*$ name, uint32_t nbc, double $*$ bounds, uint32_t $*$ counts) const

Compute the conditional distribution and return the distribution in the arrays provided.

The minimum number of bins expected is four (4). This function will return error code -1 if the value of nbc is less than 4.

3.68.3.16 long ibis::part::getCumulativeDistribution (const char $*$ name, uint32_t nbc, double $*$ bounds, uint32_t $*$ counts) const

This version of getCumulativeDistribution uses two user supplied arrays bounds and counts.

The actual number of elements filled by this function is the return value, which is guaranteed to be no larger than the input value of nbc.

3.68.3.17 long ibis::part::getCumulativeDistribution (const char $*$ constraints, const char $*$ name, std::vector $<$ double $>\&$ bounds, std::vector $<$ uint32_t $>$ \& counts) const

Compute the cumulative distribution of the variable named name under the specified constraints.

Note:

The constraints have the same syntax as the where-clause of the queries. Here are two example, "a $<5$ and 3.5 $>=\mathrm{b}>=1.9 "$ and $" \mathrm{a} * \mathrm{a}+\mathrm{b} * \mathrm{~b}>55$ and $\operatorname{sqrt}(\mathrm{c})>2$." 
3.68.3.18 long ibis::part::getCumulativeDistribution (const char $*$ name, std::vector $<$ double $>$ \& bounds, std::vector $<$ uint32_t $>$ \& counts) const

Compute a cumulative distribution (a cumulative histogram).

It returns the number of entries in arrays bounds and counts. The content of counts[i] will be the number of records in the named column that are less than bounds[i]. The last element in array bounds is larger than returned by function getColumnMax.

3.68.3.19 long ibis::part::getDistribution (const char $*$ constraints, const char $*$ name, uint32_t $n b c$, double $*$ bounds, uint32_t $*$ counts) const

Compute the conditional binned data distribution with the specified maximum number of bins.

The minimum number of bins expected is four (4). This function will return error code -1 if the value of nbc is less than 4.

3.68.3.20 long ibis::part::getDistribution (const char $*$ name, uint32_t $n b c$, double $*$ bounds, uint32_t $*$ counts) const

Compute the binned distribution with the specified maximum number of bins.

The minimum number of bins expected is four (4). This function will return error code -1 if the value of nbc is less than 4.

3.68.3.21 long ibis::part::getDistribution (const char $*$ constraints, const char $*$ name, std::vector $<$ double $>$ \& bounds, std::vector $<$ uint32_t $>$ \& counts) const

Compute the conditional binned data distribution.

If the input array bounds contains distinct values in ascending order, the array will be used as bin boundaries. Otherwise, the bin boundaries are automatically determined by this function. The basic rule for determining the number of bins is that if there are less than 10,000 distinct values, than everyone value is counted separatly, otherwise 1000 bins will be used and each bin will contain roughly the same number of records.

3.68.3.22 long ibis::part::getDistribution (const char $*$ name, std::vector $<$ double $>$ \& bounds, std::vector $<$ uint32_t $>$ \& counts) const

Compute the binned distribution of the name variable.

The array bounds defines the following bins:

(..., bounds[0]) [bounds[0], bounds[1]) ... [bounds.back ()$, \ldots$ ).

In other word, bounds[n] defines $(n+1)$ bins, with two open bins at the two ends. The array counts contains the number of rows fall into each bin. On a successful return from this function, the return value of this function is the number of bins defined, which is the same as the size of array counts but one larger than the size of array bounds.

3.68.3.23 long ibis::part::getJointDistribution (const char $*$ constraints, const char $*$ name1, const char $*$ name 2 , std::vector $<$ double $>\&$ bounds 1 , std::vector $<$ double $>\&$ bounds 2 , std::vector $<$ uint32_t $>\&$ counts) const

Compute the joint distribution of two variables.

It returns three arrays, bounds 1 , bounds 2 , and counts. The arrays bounds 1 andbounds 2 defines two sets of bins one for each variable. Together they define

(bounds1.size ()+1)

(bounds2.size ()+1) 
bins for the 2-D joint distributions. The array count s contains a count for each of the bins. On successful completion of this function, it return the number of bins.

\subsubsection{4 const std::vector $<$ uint32_t $>$ \& ibis::part::getMeshShape () const [ in l ine ]}

In many scientific applications, data are defined on meshes.

The following functions assumes the meshes are regular. Under this assumption, each column can be viewed as a multi-dimensional array, such as A[nz][ny][nx]. Following the convention in $\mathrm{C} / \mathrm{C}++$. The dimensions of the array are ordered from left to the right, with the left most being the slowest varying dimension and the right most being the fast varying dimension. This assumption about the dimensions is explicitly used in query.cpp in functions toRanges, range $2 \mathrm{~d}$, range $3 \mathrm{~d}$ and rangend. The function getMeshShape returns the sizes of the dimensions in a vector.

\subsubsection{5 float ibis::part::getUndecidable (const ibis::qContinuousRange \& $c m p$, ibis::bitvector \& iffy) const}

Discover the records that can not be decided using the index.

Logically, iffy = high - low, were high and low are computed from estimateRange. The return value is the estimated fraction of records that might satisfy the range condition.

\subsubsection{6 void ibis::part::loadIndex (const char $*$ opt $=0)$ const}

Load all indices.

If none existed, build them. If an index exists already, it reads metadata about the index into memory. Newly built indices are completely in memory. An index can not be built correctly if it is too large to fit in memory!

\subsubsection{7 long ibis::part::lookforString (const ibis::qMultiString \& cmp, ibis::bitvector \& low) const}

Determine the records that have the exact string values.

Actual work done in the function search of the string-valued column. It produces no hit if the name is not a stringvalued column.

3.68.3.28 long ibis::part::matchAny (const ibis::qAnyAny \& cmp, const ibis::bitvector \& mask, ibis::bitvector \& hits) const [virtual]

Perform exact match operation for an AnyAny query.

The bulk of the work is performed as range query.

\subsubsection{9 std::string ibis::part::metaTags () const}

Return the list of meta tags as a single string.

The meta tags appears as 'name=value' pairs separated by comma (,).

\subsubsection{0 void ibis::part::purgeIndexFiles () const}

Remove existing index files!

This function is useful after changing the index specification before rebuilding a set of new indices.

3.68.3.31 int ibis::part::readTDC (size_t \& nrows, columnList \& plist, const char $*$ dir) $\quad$ [protected ] Read TDC file. 
If dir is the activeDir, it will also update the content of $*$ this, otherwise it will only modify arguments nrows and plist. If this function completes successfully, it returns the maximum length of the column names. Otherwise, it returns a value of zero or less to indicate errors.

\section{Remarks:}

The metadata file is named "-part.txt". It is a plain text file with a fixed structure. The prefix '-' is to ensure that none of the data files could possibly have the same name (since '-' cann't appear in any column names). This file was previously named "table.tdc" and this function still recognize this old name.

\subsubsection{2 long ibis::part::reorder ()}

Reorder all columns of a partition.

The lowest cardinality column is ordered first. Only integral valued columns are used in sorting. Returns the number of rows reordered when successful, otherwise return a negative number and the base data is corrupt! Danger: This function does not update null masks!

\subsubsection{3 long ibis::part::rollback ()}

Rollback(revert) to previous data set.

Can only undo the last change to the database, currently only function append can change the database.

\subsubsection{4 void ibis::part::setMeshShape (const char $*$ shape) [inline]}

Digest the mesh shape stored in the string.

The shape can be just numbers, e.g., " $(10,12,14)$ ", or 'name=value' pairs, e.g., "(nz=10, ny=12, nx=14)".

\subsubsection{5 void ibis::part::setMetaTags (const std::vector $<$ const char $*>\boldsymbol{\&} \boldsymbol{m t s}$ ) [protected]}

Make a deep copy of the incoming name-value pairs.

The even element is assumed to be the name and the odd element is assumed to be the value. If the last name is not followed by a value it is assumed to have the value of ' $*$ ', which indicates do-not-care.

The documentation for this class was generated from the following files:

- part.h

- part.cpp

- parti.cpp

- party.cpp

\subsection{9 ibis::part::advisoryLock Class Reference}

An non-blocking version of writeLock.

\#include <part.h>

\section{Public Member Functions}

- bool acquired () const

- advisoryLock (const part $* \mathrm{tbl}$, const char $* \mathrm{~m}$ ) 


\subsubsection{Detailed Description}

An non-blocking version of writeLock.

The function acquired returns true is the object has acquired a lock successfully, otherwise the function returns false.

The documentation for this class was generated from the following file:

- part.h

\subsection{0 ibis::part::barrel Class Reference}

To read a list of variables at the same time.

\#include <part.h>

Inheritance diagram for ibis::part::barrel::

\begin{tabular}{|l|}
\hline ibis::part::barrel \\
\hline ibis::part::vault \\
\hline
\end{tabular}

\section{Public Member Functions}

- barrel (const ibis::part $* \mathrm{t}=0$ )

- virtual long close ()

Close all open files.

- const ibis::column $*$ getColumn (uint32_t i) const

- void getNullMask (ibis::bitvector \&mask) const

- virtual long open (const ibis::part $* \mathrm{t}=0$ )

Open all data files.

- virtual long read ()

Read one value for each variable.

- virtual long seek (uint32_t pos)

Move the file pointers to the posth record.

- uint32_t tell () const

- virtual barrel ()

Destructor closes the open files.

\section{Protected Attributes}

- const ibis::part $*$ _tbl

- std::vector $<$ const ibis::column $*>$ cols

- std::vector $<$ int $>$ fdes

- uint32_t position

- std::vector < ibis::fileManager::storage $*>$ stores 


\subsubsection{Detailed Description}

To read a list of variables at the same time.

This implementation opens each data file and read the values from the files one at a time.

\subsubsection{Member Function Documentation}

3.70.2.1 long ibis::part::barrel::read () [virtual]

Read one value for each variable.

All values are internally stored as doubles.

Reimplemented in ibis::part::vault.

\subsubsection{2 long ibis::part::barrel::seek (uint32_t pos) [virtual]}

Move the file pointers to the posth record.

Return 0 for success and other integer for error.

Reimplemented in ibis::part::vault.

The documentation for this class was generated from the following files:

- part.h

- part.cpp

\subsection{1 ibis::part::cleaner Class Reference}

A cleaner to be used by the fileManager::unload function.

\#include <part.h>

\section{Public Member Functions}

- cleaner (const part $*$ tbl)

- virtual void operator() () const

\subsubsection{Detailed Description}

A cleaner to be used by the fileManager::unload function.

The documentation for this class was generated from the following file:

- part.h

\subsection{2 ibis::part::info Struct Reference}

A simple class to describe an ibis::part object.

\#include <part.h>

\section{Public Member Functions}

- info (const ibis::part \&tbl)

- info (const char $*$ na, const char $*$ de, const uint64_t \&nr, const ibis::part::columnList \&co) 


\section{Public Attributes}

- std::vector < ibis::column::info $*>$ cols

The list of columns in the partition.

- const char $*$ description

A free-form description of the partition.

- const char $*$ metaTags

A string of name-value pairs.

- const char $*$ name

Partition name.

- const uint64_t nrows

The number of rows in the partition.

\subsubsection{Detailed Description}

A simple class to describe an ibis::part object.

All members are public and read-only. An info object can not last longer than the ibis::part object used to create it.

The documentation for this struct was generated from the following files:

- part.h

- part.cpp

\subsection{3 ibis::part::mutexLock Class Reference}

Provide a mutual exclusion lock on an ibis::part object.

\#include <part.h>

\section{Public Member Functions}

- mutexLock (const part $*$ tbl, const char $* \mathrm{~m})$

\subsubsection{Detailed Description}

Provide a mutual exclusion lock on an ibis::part object.

The documentation for this class was generated from the following file:

- part.h

\subsection{4 ibis::part::readLock Class Reference}

Provide a read lock on an ibis::part.

\#include <part.h> 


\section{Public Member Functions}

- readLock (const part $*$ tbl, const char $* \mathrm{~m})$

\subsubsection{Detailed Description}

Provide a read lock on an ibis::part.

Routines need read access to ibis::part class should use this class instead directly calling ibis::partgainReadAccess so that in case of exceptions, the release command would be always called.

The documentation for this class was generated from the following file:

- part.h

\subsection{5 ibis::part::vault Class Reference}

To read variables in certain order.

\#include <part.h>

Inheritance diagram for ibis::part::vault::

\begin{tabular}{|l|}
\hline ibis::part::barrel \\
\hline ibis::part::vault \\
\hline
\end{tabular}

\section{Public Member Functions}

- virtual long open (const ibis::part $* \mathrm{t}=0$ )

Open all data files.

- virtual long read ()

Read the values at the current position.

- long seek (double val)

Move to the first position that value(var) $>=$ val.

- virtual long seek (uint32_t pos)

Move the logical position.

- uint32_t tellReal () const

Tell the physical record number.

- vault (const ibis::roster \&r)

\subsubsection{Detailed Description}

To read variables in certain order.

A version of barrel that keys on an index array (i.e., a roster). 


\subsubsection{Member Function Documentation}

\subsubsection{1 long ibis::part::vault::read () [virtual]}

Read the values at the current position.

Treat position as the logical position, the physical position is_roster[position].

Reimplemented from ibis::part::barrel.

\subsubsection{2 uint32_t ibis::part::vault::tellReal () const}

Tell the physical record number.

User may called_roster[tell()] to avoid the overhead of calling this function.

The documentation for this class was generated from the following files:

- parth

- part.cpp

\subsection{6 ibis::part::writeLock Class Reference}

Provide a write lock on an ibis::part.

\#include <part.h>

\section{Public Member Functions}

- writeLock (const part $*$ tbl, const char $* \mathrm{~m}$ )

\subsubsection{Detailed Description}

Provide a write lock on an ibis::part.

The documentation for this class was generated from the following file:

- parth

\subsection{7 ibis::qAnyAny Class Reference}

A user specifies this type of query expression with the following syntax,

\#include <qExpr.h>

Inheritance diagram for ibis::qAnyAny::

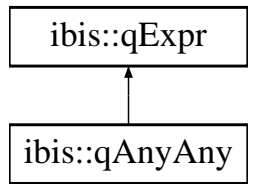

Public Member Functions

- virtual qExpr $*$ dup () const

- const char $*$ getPrefix () const 
- const std::vector $<$ double $>\&$ getValues () const

- virtual void print (std::ostream \&out) const

Print out the node in the string form.

- virtual void printRange (std::ostream \&out) const

Print out the attribute name and the constants involved in the range expressions.

- qAnyAny (const char $*$ pre, const char $*$ val)

Constructing an object of type qAnyAny from two strings.

\subsubsection{Detailed Description}

A user specifies this type of query expression with the following syntax,.

- $\operatorname{any}($ prefix $)=$ value

- any(prefix) in (list of values) If any column with the given prefix contains the specified values, the row is considered as a hit. This is intended to be used in the cases where the prefix is actually the name of a setvalued attribute, such as triggerID in STAR datasets. In this case, the set-valued attribute is translated into a number of columns with the same prefix. A common query is "does the set contain a particular value?" or "does the set contain a particular set of values?"

\subsubsection{Member Function Documentation}

\subsubsection{1 void ibis::qAnyAny::printRange (std::ostream \& out) const [virtual]}

Print out the attribute name and the constants involved in the range expressions.

Only print something is the node is a qRange.

Reimplemented from ibis::qExpr.

The documentation for this class was generated from the following files:

- qExpr.h

- qExpr.cpp

\subsection{8 ibis::qContinuousRange Class Reference}

Simple range condition.

\#include <qExpr.h>

Inheritance diagram for ibis::qContinuousRange::

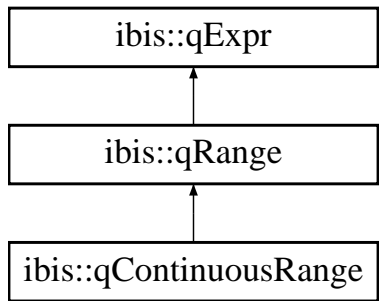




\section{Public Member Functions}

- virtual const char $*$ colName () const

Returns the name of the attribute involved.

- virtual qContinuousRange $*$ dup () const

- virtual bool empty () const

$$
\text { Is the current range empty? }
$$

- void foldBoundaries ()

- void foldUnsignedBoundaries ()

- virtual bool inRange (double val) const

Given a value, determine whether it is in the range defined.

- double \& leftBound ()

- virtual const double \& leftBound () const

The lower bound of the range.

- COMPARE \& leftOperator ()

- COMPARE leftOperator () const

- bool operator< (const qContinuousRange \&y) const

The operator $<$ for ibis::qContinuousRange.

- virtual void print (std::ostream \&) const

Print out the node in the string form.

- virtual void printRange (std::ostream \&out) const

Print out the attribute name and the constants involved in the range expressions.

- qContinuousRange (const char $*$ prop, COMPARE op, double val)

- qContinuousRange (double lv, COMPARE lop, const char *prop, COMPARE rop, double rv)

- qContinuousRange (const qContinuousRange \&rhs)

- qContinuousRange (const char $*$ col, COMPARE op, uint32_t val)

- qContinuousRange (const char $* 1$ str, COMPARE lop, const char $*$ prop, COMPARE rop, const char $*$ rstr)

- virtual void restrictRange (double left, double right)

Reduce the range to be no more than [left, right].

- double \& rightBound ()

- virtual const double \& rightBound () const

The upper bound of the range.

- COMPARE \& rightOperator ()

- COMPARE rightOperator () const

\subsubsection{Detailed Description}

Simple range condition.

It is implemented as a derived class of qExpr. Possible range operator are defined in ibis::qExpr::COMPARE. 


\subsubsection{Member Function Documentation}

3.78.2.1 bool ibis::qContinuousRange::inRange (double val) const [inline, virtual]

Given a value, determine whether it is in the range defined.

Return true if it is, return false, otherwise.

Implements ibis::qRange.

\subsubsection{2 void ibis::qContinuousRange::printRange (std::ostream \& out) const [virtual]}

Print out the attribute name and the constants involved in the range expressions.

Only print something is the node is a qRange.

Reimplemented from ibis::qExpr.

The documentation for this class was generated from the following files:

- qExpr.h

- qExpr.cpp

\subsection{9 ibis::qExpr Class Reference}

The top level query expression object.

\#include <qExpr.h>

Inheritance diagram for ibis::qExpr::

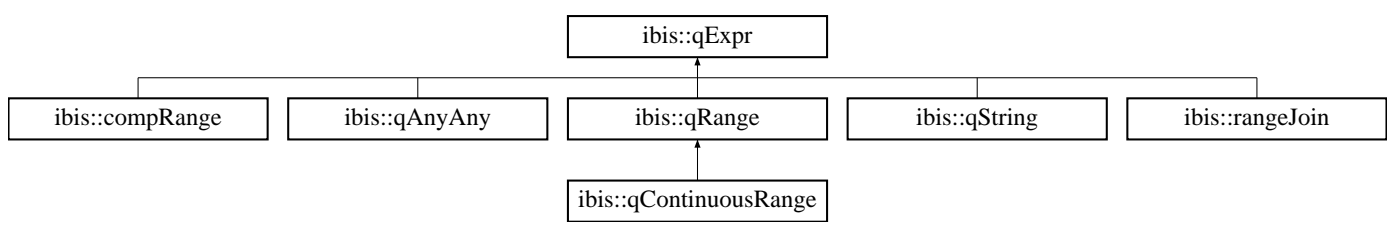

\section{Public Types}

- enum COMPARE \{

OP_UNDEFINED, OP_LT, OP_GT, OP_LE, OP_GE, OP_EQ \}

Comparison operator supported in RANGE.

- enum TYPE \{

LOGICAL_UNDEFINED, LOGICAL_NOT, LOGICAL_AND, LOGICAL_OR,

LOGICAL_XOR, LOGICAL_MINUS, RANGE, DRANGE,

STRING, MSTRING, COMPRANGE, MATHTERM,

JOIN, TOPK, ANYANY \}

Definition of node types.

Public Member Functions

- virtual qExpr $*$ dup () const 
- void extractJoins (std::vector $<$ const rangeJoin $*>$ \&terms) const

Extract conjunctive terms of the specified type.

- qRange $*$ findRange (const char $*$ vname)

Find the first range condition involving the named variable.

- const qExpr $*$ getLeft () const

Return a const pointer to the left child.

- $\mathrm{qExpr} * \&$ getLeft ()

Return a pointer to the left child.

- const qExpr $*$ getRight () const

Return a const pointer to the right child.

- $\mathrm{qExpr} * \&$ getRight ()

Return a pointer to the right child.

- TYPE getType () const

Return the node type.

- bool hasJoin () const

Return true is there is a term with join operation, false otherwise.

- virtual bool isSimple () const

Is the expression simple, i.e., containing only simple range conditions joined with logical operators?

- const qExpr \& operator= (const qExpr \&rhs)

- virtual void print (std::ostream \&) const

Print out the node in the string form.

- virtual void printRange (std::ostream \&out) const

Print out the attribute name and the constants involved in the range expressions.

- qExpr (const qExpr \&qe)

Deep copy.

- $\mathrm{qExpr}$ (TYPE op, qExpr *qe1, qExpr *qe2)

Construct a full specified node. All three arguments are present.

- qExpr (TYPE op)

Construct a node of specified type. Not for implicit type conversion.

- $\mathrm{q} \operatorname{Expr}()$

Default constructor. It generates a node of undefined type.

- double reorder (const weight \&)

Reorder the expressions tree.

- int separateSimple (ibis::qExpr $* \&$ simple, ibis::qExpr $* \&$ tail) const

Separate an expression tree into two joined with an AND operator.

- void setLeft (qExpr *expr) 
Change the left child.

- void setRight (qExpr *expr)

Change the right child.

- virtual qExpr ()

Destruct the node recursively.

\section{Static Public Member Functions}

- static void simplify (ibis::qExpr $* \&$ )

Attempt to convert simple compRanges into qRanges.

\section{Classes}

- struct weight

A functor to be used by the function reorder.

\subsubsection{Detailed Description}

The top level query expression object.

It encodes the logical operations between two child expressions. It is to serve as the interior nodes of an expression tree. Leaf node are going to be derived later.

\subsubsection{Member Enumeration Documentation}

\subsubsection{1 enum ibis::qExpr::TYPE}

Definition of node types.

Logical operators are listed in the front and leaf node types are listed at the end.

\subsubsection{Member Function Documentation}

\subsubsection{1 virtual void ibis::qExpr::printRange (std::ostream \& out) const [inline, virtua l]}

Print out the attribute name and the constants involved in the range expressions.

Only print something is the node is a qRange.

Reimplemented in ibis::qContinuousRange, and ibis::qAnyAny.

\subsubsection{2 int ibis::qExpr::separateSimple (ibis::qExpr $* \&$ simple, ibis::qExpr $* \&$ tail) const}

Separate an expression tree into two joined with an AND operator.

The first one of the two new expressions contains only simple range expressions, and the second contains what is left. 


\subsubsection{3 void ibis::qExpr::simplify (ibis::qExpr $* \boldsymbol{\&}$ ) [ stat ic ]}

Attempt to convert simple compRanges into qRanges.

This is necessary because the parser always generates compRange instead of qRange.

The documentation for this class was generated from the following files:

- qExpr.h

- qExpr.cpp

\subsection{0 ibis::qExpr::weight Struct Reference}

A functor to be used by the function reorder.

\#include <qExpr.h>

\section{Public Member Functions}

- virtual double operator() (const qExpr $*$ ex) const=0

\subsubsection{Detailed Description}

A functor to be used by the function reorder.

The documentation for this struct was generated from the following file:

- qExpr.h

\subsection{1 ibis::qRange Class Reference}

A class to represent simple range conditions.

\#include <qExpr.h>

Inheritance diagram for ibis::qRange::

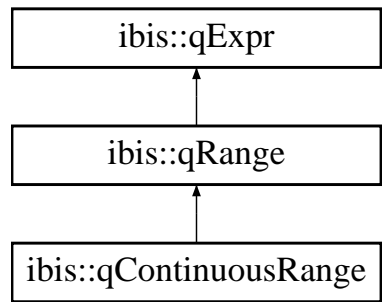

\section{Public Member Functions}

- virtual const char $*$ colName () const $=0$

Returns the name of the attribute involved.

- virtual bool empty () const=0

Is the current range empty?

- virtual bool inRange (double val) const $=0$

Given a value, determine whether it is in the range defined. 
- virtual const double \& leftBound () const=0

The lower bound of the range.

- virtual void restrictRange (double left, double right $)=0$

Reduce the range to be no more than [left, right].

- virtual const double \& rightBound () const=0

The upper bound of the range.

\section{Protected Member Functions}

- qRange (TYPE t)

\subsubsection{Detailed Description}

A class to represent simple range conditions.

This is an abstract base class for qContinuousRange and qDiscreteRange. The two main virtual functions defined in this class are used by the procedures that evaluate the conditions.

\subsubsection{Member Function Documentation}

\subsubsection{1 virtual bool ibis::qRange::inRange (double $\boldsymbol{v a l}$ ) const [pure virtual]}

Given a value, determine whether it is in the range defined.

Return true if it is, return false, otherwise.

Implemented in ibis::qContinuousRange.

The documentation for this class was generated from the following file:

- qExpr.h

\subsection{2 ibis::qString Class Reference}

The class qString encapsulates information for comparing string values.

\#include <qExpr.h>

Inheritance diagram for ibis::qString::

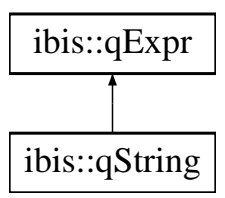

\section{Public Member Functions}

- virtual qString $*$ dup () const

- const char $*$ leftString () const

- virtual void print (std::ostream \&) const 
Print out the node in the string form.

- $\mathbf{q S t r i n g}$ (const char $* \mathrm{ls}$, const char $* \mathrm{rs}$ )

- const char $*$ rightString () const

\subsubsection{Detailed Description}

The class qString encapsulates information for comparing string values.

Only equality comparison is supported at this point. It does not ensure the names are valid in any way. When the check does happen, the left side will be checked first. If it matches the name of a ibis::column, the right side will be assumed to be the value one is trying to match. If the left side does not match any know column name, but the right side does, the right side will be assumed to name of column to be searched and the left side will be the value to search against. If neither matches the name of any column, the expression will evaluate to NULL (i.e., no hit).

The documentation for this class was generated from the following files:

- qExpr.h

- qExpr.cpp

\subsection{3 ibis::query Class Reference}

A data structure for representing user queries.

\#include <query.h>

Inheritance diagram for ibis::query::

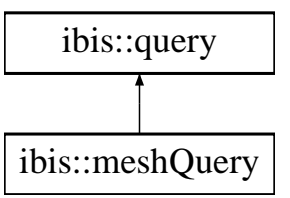

\section{Public Types}

- enUm QUERY_STATE \{

UNINITIALIZED, SET_COMPONENTS, SET_RIDS, SET_PREDICATE,

SPECIFIED, QUICK_ESTIMATE, FULL_EVALUATE, BUNDLES_TRUNCATED, HITS_TRUNCATED \}

\section{Public Member Functions}

- void clear ()

Releases the resources held by the query object and re-initialize the select clause and the where clause to blank.

- void clearErrorMessage () const

Reset the last error message to blank.

- const selected \& components () const

Return a list of names specified in the select clause.

- void contractQuery ()

Contracts where clause to preferred bounds. 
- long countHits () const

Count the number of hits.

- const part $*$ dataTable () const

Return the pointer to the data table used to process the query.

- const char $* \operatorname{dir}()$ const

The query token. For persistent data.

- int estimate ()

Functions to perform estimation and retrieve range of hits Computes a lower and an upper bound of hits.

- int evaluate (const bool evalSelect=false)

Computes the exact hits.

- void expandQuery ()

Expands where clause to preferred bounds.

- const ibis::bitvector $*$ getHitVector () const

Return the pointer to the internal hit vector.

- const char $*$ getLastError () const

Return the last error message recorded internally.

- long getMaxNumHits () const

Return the number of records in the upper bound.

- long getMinNumHits () const

Return the number of records in the lower bound.

- long getNumHits () const

Return the number of records in the exact solution.

- array_t $<$ double $>*$ getQualifiedDoubles (const char $*$ column_name)

- array_t $<$ float $>*$ getQualifiedFloats (const char $*$ column_name)

- $\operatorname{array} \_\mathrm{t}<$ int32_t $>*$ getQualifiedInts (const char $*$ column_name)

The functions getQualifiedXXX return the values of selected columns in the records that satisfies the specified conditions.

- array_t $<$ uint32_t $>*$ getQualifiedUInts (const char $*$ column_name)

- RIDSet $*$ getRIDs (const ibis::bitvector \&mask) const

Return a list of row ids that match the mask.

- RIDSet $*$ getRIDs () const

Return the list of row IDs of the hits.

- const RIDSet $*$ getRIDsInBundle (const uint32_t bid) const

Return the list of row IDs of the hits within the specified bundle.

- virtual const char $*$ getSelectClause () const

Return the select clause string. 
- QUERY_STATE getState () const

Return the current state of query.

- const RIDSet $*$ getUserRIDs () const

Return a const pointer to the copy of the user supplied RID set.

- const char $*$ getWhereClause () const

Return the where clause string.

- const char $*$ id () const

Functions about the identity of the query.

- long int limit (const char $*$ names, int direction, uint32_t keep, bool updateHits=true)

Truncate the bundles to provide the top-K rows of the bundles.

- void logMessage (const char $*$ event, const char $* \mathrm{fmt}, \ldots$ ) const

Used to print information about the progress or state of query processing.

- int orderby (const char $*$ names, int direction) const

Re-order the bundles according the the new "ORDER BY" specification.

- void printSelected (std::ostream \&out) const

Print the values of the selected columns to the specified output stream.

- void printSelectedWithRID (std::ostream \&out) const

Print the values of the columns in the select clause without functions.

- query (const char $*$ uid $=0$, const part $* \mathrm{et}=0$, const char $*$ pref $=0$ )

Constructor. Generates a new query on the given table et.

- query (const char $*$ dir, const ibis::partList \&tl)

Constructor.

- RIDSet $*$ readRIDs () const

- std::string removeComplexConditions ()

Separate out the sub-expressions that are not simple.

- ibis::bitvector $*$ sequentialScan () const

Return a (new) bitvector that contains the result of directly scan the raw data to determine what records satisfy the user specified conditions.

- int setRIDs (const RIDSet \&set)

Specifies a list of Row IDs for the query object to retrieve the records.

- virtual int setSelectClause (const char $*$ str)

Specifies the select clause for the query.

- int setTable (const ibis::part $*$ tbl)

Resets the table used to evaluate the query conditions to the table specified in the argument.

- int setWhereClause (const char $*$ str)

Specifies the where clause for the query. 
- time_t timestamp () const

The time stamp on the data used to process the query.

- const char $*$ userName () const

User started the query.

- void writeRIDs (const RIDSet $*$ rids) const

- template $<>$ int64_t countDeltaPairs (const array_t $<$ int32_t $>$ \&val1, const array_t $<$ uint32_t $>$ \&val2, const int32_t \&delta) const

- template $<>$ int64_t countDeltaPairs (const array_t $<$ uint32_t $>$ \&val1, const array_t $<$ int32_t $>$ \&val2, const uint32_t \&delta) const

- template $<>$ int64_t countEqualPairs (const array_t $<$ uint32_t $>$ \&val1, const array_t $<$ int32_t $>$ \&val2) const

- template $<>$ int64_t countEqualPairs (const array_t $<$ int32_t $>$ \&val1, const array_t $<$ uint32_t $>$ \&val2) const

This is an explicit specialization of a protected member of ibis::query class.

- template $<>$ int64_t recordDeltaPairs (const array_t $<$ int32_t $>$ \&val1, const array_t $<$ uint32_t $>$ \&val2, const array_t $<$ uint32_t $>$ \&ind1, const array_t $<$ uint32_t $>$ \&ind2, const int32_t \&delta, const char $*$ filename) const

- template $<>$ int64_t recordDeltaPairs (const array_t $<$ uint32_t $>$ \&val1, const array_t $<$ int32_t $>$ \&val2, const array_t $<$ uint32_t $>$ \&ind1, const array_t $<$ uint32_t $>$ \&ind2, const uint32_t \&delta, const char $*$ filename) const

- template $<>$ int64_t recordEqualPairs (const array_t $<$ int32_t $>$ \&val1, const array_t $<$ uint32_t $>$ \&val2, const array_t $<$ uint32_t $>$ \&ind1, const array_t $<$ uint32_t $>$ \&ind2, const char $*$ filename) const

- template $<>$ int64_t recordEqualPairs (const array_t $t<$ uint32_t $>$ \&val1, const array_t $<$ int32_t $>$ \&val2, const array_t $<$ uint32_t $>$ \&ind1, const array_t $<$ uint32_t $>$ \&ind2, const char $*$ filename) const

\section{Static Public Member Functions}

- static bool isValidToken (const char *tok)

Is the given string a valid query token.

- static void keepQueryRecords ()

Tell the destructor to leave stored information on disk.

- static void removeQueryRecords ()

Tell the destructor to remove all stored information about queries.

- static unsigned tokenLength ()

Length of the query token.

\section{Protected Member Functions}

- void addJoinConstraints (ibis::qExpr $* \& \exp 0$ ) const

Add constraints derived from domains of the two join columns.

- void computeHits ()

- template $<$ typename T1, typename T2 $>$ int64_t countDeltaPairs (const array_t $<\mathrm{T} 1>\&$ val1, const array_t $<$ $\mathrm{T} 2>$ \&val2, const T1 \&delta) const

Assume the two input arrays are sorted in ascending order, count the number of elements that are with delta of each other. 
- template $<$ typename T1, typename T2 $>$ int64_t countEqualPairs (const array_t $<\mathrm{T} 1>\&$ val1, const array_t $<$ $\mathrm{T} 2>\&$ val2) const

Assume the two input arrays are sorted in ascending order, count the number of elements that match.

- uint32_t countPages (unsigned wordsize) const

- int doContract (ibis::qExpr *exp0) const

- void doEstimate (const qExpr *term, ibis::bitvector \&low, ibis::bitvector \&high) const

- void doEvaluate (const qExpr *term, const ibis::bitvector \&mask, ibis::bitvector \&hits) const

- void doEvaluate (const qExpr *term, ibis::bitvector \&hits) const

- int doExpand (ibis::qExpr *exp0) const

- void doScan (const qExpr $*$ term, ibis::bitvector \&hits) const

- void doScan (const qExpr *term, const ibis::bitvector \&mask, ibis::bitvector \&hits) const

- void gainReadAccess (const char $*$ mesg) const

- void gainWriteAccess (const char $*$ mesg) const

- void getBounds ()

- bool hasBundles () const

- void logError (const char $*$ event, const char $* \mathrm{fmt}, \ldots$..) const

- void logWarning (const char $*$ event, const char $* \mathrm{fmt}, \ldots$.) const

- int64_t mergePairs (const char $*$ pairfile) const

- void orderPairs (const char $*$ pairfile) const

Sort the content of the file as ibis: :rid_t.

- void printRIDs (const RIDSet \& ridset) const

This function prints a list of RIDs to st dout.

- int64_t processJoin ()

Process the join operation and return the number of pairs.

- void readHits ()

- void readQuery (const ibis::partList \&tl)

- template $<$ typename T1, typename T2 $>$ int64_t recordDeltaPairs (const array_t $<\mathrm{T} 1>$ \& val1, const array_t $<$ $\mathrm{T} 2>$ \&val2, const array_t $<$ uint32_t $>$ \&ind1, const array_t $<$ uint32_t $>$ \&ind2, const T1 \&delta, const char $*$ pairfile) const

- template $<$ typename T1, typename $\mathrm{T} 2>$ int64_t recordEqualPairs (const array_t $<\mathrm{T} 1>\&$ val1, const array_$\mathrm{t}<\mathrm{T} 2>$ \&val2, const array_t $<$ uint32_t $>$ \&ind1, const array_t $<$ uint32_t $>$ \&ind2, const char $*$ pairfile) const

- void releaseAccess (const char $*$ mesg) const

- void removeFiles ()

- void reorderExpr ()

- int64_t sortEquiJoin (const ibis::rangeJoin \&cmp, const ibis::bitvector \&mask, const char $*$ pairfile) const

Perform equi-join by sorting the selected values.

- int64_t sortEquiJoin (const ibis::rangeJoin \&cmp, const ibis::bitvector \&mask) const

Performing an equi-join by sorting the selected values first.

- int64_t sortJoin (const ibis::rangeJoin \&cmp, const ibis::bitvector \&mask) const

- int64_t sortJoin (const std::vector < const ibis::rangeJoin $*>$ \&terms, const ibis::bitvector \&mask) const

- int64_t sortRangeJoin (const ibis::rangeJoin \&cmp, const ibis::bitvector \&mask, const char $*$ pairfile) const

Performing range join by sorting the selected values.

- int64_t sortRangeJoin (const ibis::rangeJoin \&cmp, const ibis::bitvector \&mask) const

Performing a range join by sorting the selected values.

- int verifyPredicate (qExpr $* \& q$ expr)

- void writeHits () const

- virtual void writeQuery () 


\section{Protected Attributes}

- selected comps

Names of selected components.

- char $*$ condition

Query condition (string).

- ibis::part::readLock $*$ dslock

A read lock on the table0.

- ibis::bitvector $*$ hits

Solution in bitvector form (or lower bound).

- char lastError [MAX_LINE+PATH_MAX]

The warning/error message.

- QUERY_STATE state

Status of the query.

- ibis::bitvector $*$ sup

Estimated upper bound.

- char $*$ user

Name of the user who specified the query.

\section{Friends}

- class readLock

- class writeLock

\section{Classes}

- class readLock

- class result

The class ibis::query::result allows user to retrieve query result one row at a time.

- class weight

- class writeLock

\subsubsection{Detailed Description}

A data structure for representing user queries.

This is the primary entry for user to take advantage of bitmap indexing facilities. A query is a very limited version of the SQL SELECT statement. It is only defined on one table and it takes a where clause and a select clause. The where clause is mandatory. It contains a list of range conditions joined together with logical operators, such as "temperature $>700$ and $100<=$ presessure $<350 "$. Records whose attribute values satisfy the conditions defined in the where clause is considered hits. A query may retrieve values of variables/columns specified in the select clause. A select clause is optional. If specified, it contains a list of column names. These attributes must not be NULL in order for a record to be a hit. The select clause may also contain column names appearing as the argument to one of the four functions: avg, max, min and sum. For example, "temperature, pressure, average(ho2_concentration)" may be a select statement for a Chemistry application. 
The hits can be computed in two ways by using functions estimate or evaluate. The function estimate can take advantage of the indices to give two approximate solutions, one as an upper bound and the other as a lower bound. The bitmap indices will be automatically built according to the specification if they are not present. The accuracy of the bounds depend on the nature of the indices available. If no index can be constructed, the lower bound would be empty and the upper bound would include every record. When the function evaluate is called, the exact solution is computed no matter whether the function estimate has been called or not. The solution produced is recorded as a bit vector. The user may use ibis: :bitvector: : indexset to extract the record numbers of the hits or use one of the functions getQualifiedInts, getQualifiedFloats, and getQualifiedDoubles to retrieve the values of the selected attributes. Additionally, one may call either print Selected or print SelectedWithRID to print the selected values to the specified I/O stream.

\subsubsection{Constructor \& Destructor Documentation}

\subsubsection{1 ibis::query::query (const char $*$ dir, const ibis::partList \& $t l$ )}

Constructor.

Reconstructs query from stored information in the named directory dir. This is only used for recovering from program crashes.

\subsubsection{2 ibis::query::query (const char $*$ uid $=0$, const part $* e t=0$, const char $*$ pref $=0$ )}

Constructor. Generates a new query on the given table et.

If recovery is desired or the query objects has its own special prefix, a cache directory is created to store some information about the query such as the query conditions and the resulting solutions. The stored information enables it to be reconstructed in case of crash.

\subsubsection{Member Function Documentation}

\subsubsection{1 void ibis::query::contractQuery ()}

Contracts where clause to preferred bounds.

Similar to function exandQuery, but makes the bounds of the range conditions narrower rather than wider.

3.83.3.2 template $<$ typename T1, typename T2 $>$ int64_t ibis::query::countDeltaPairs (const array_t $<$ T1 $>$ \& val1, const array_t $<\mathbf{T} 2>\&$ val2, const $\mathbf{T} 1 \&$ delta) const [ protected]

Assume the two input arrays are sorted in ascending order, count the number of elements that are with delta of each other.

Note that both template arguments should be elemental types or they must support operators,,$-+==$ and $<$ with mixed types.

3.83.3.3 int64_t ibis::query::countEqualPairs (const array_t $<$ int32_t $>$ \& val1, const array_t $<$ uint32_t $>$ \& val2) const

This is an explicit specialization of a protected member of ibis::query class.

Note:

The $\mathrm{C}++$ language rules require explicit specialization of template member function be declared in the namespace containing the function, not inside the class! This apparently causes them to be listed as public functions in Doxygen document. 
3.83.3.4 template $<$ typename T1, typename T2 $>$ int64_t ibis::query::countEqualPairs (const array_t $<$ T1 $>$ $\boldsymbol{\&}$ val1, const array_t $<\mathbf{T 2}>\boldsymbol{\&}$ val2) const [protected]

Assume the two input arrays are sorted in ascending order, count the number of elements that match.

Note that both template arguments should be elemental types or they must support operators $==$ and $<$ with mixed types.

\subsubsection{5 long ibis::query::countHits () const}

Count the number of hits.

Don't generate the hit vector if not already there.

\subsubsection{6 int ibis::query::estimate ()}

Functions to perform estimation and retrieve range of hits Computes a lower and an upper bound of hits.

This is done by using the indices. If possible it will build new indices. The lower bound contains only records that are hits and the upper bound contains all hits but may also contain some records that are not hits. Returns 0 for success, a negative value for error.

\subsubsection{7 int ibis::query::evaluate (const bool evalSelect $=$ fal se)}

Computes the exact hits.

The same answer shall be computed whether there is any index or not. The argument evalSelect indicates whether the select clause should be evaluated at the same time. If its value is true, the columns specified in the select clause will be retrieved from disk and stored in the temporary location for this query. If not, the qualified values will be retrieved from disk when one of getRIDs, getQualifiedInts, getQualifiedFloats, and getQualifiedDoubles is issued. In the later case, only the specified column is retrieved. In addition, the values of column at the time of the function are read, which can be potentially different different from the time when the function evaluate was called.

Returns 0 for success, a negative value for error.

\section{See also:}

getQualifiedInts

\subsubsection{8 void ibis::query::expandQuery ()}

Expands where clause to preferred bounds.

This is to make sure the function estimate will give exact answer. It does nothing if there is no preferred bounds in the indices.

\subsubsection{9 const ibis::bitvector* ibis::query::getHitVector () const [inline ]}

Return the pointer to the internal hit vector.

The user should NOT attempt to free the returned pointer.

\subsubsection{0 array_t $<$ int32_t $>*$ ibis::query::getQualifiedInts (const char $*$ column_name)}

The functions getQualifiedXXX return the values of selected columns in the records that satisfies the specified conditions.

The caller must call the operator delete to free the pointers returned. 
Note:

Any column of the table may be specified, not just those given in the select clause. The content returned is read from disk when these functions are called, which may be different from their values when the function evaluate was called. In other word, they may be inconsistent with the conditions specified in the where clause. For append-only data, this is NOT an issue.

The above caveat also applies to the two versions of getRIDs.

\subsubsection{1 ibis::RIDSet $*$ ibis::query::getRIDs (const ibis::bitvector \& mask) const}

Return a list of row ids that match the mask.

The user is responsible for freeing the pointer.

See also:

getQualifiedInts

\subsubsection{2 ibis::RIDSet $*$ ibis::query::getRIDs () const}

Return the list of row IDs of the hits.

The user is responsible for freeing the pointer.

\section{See also:}

getQualifiedInts

\subsubsection{3 bool ibis::query::isValidToken (const char $*$ tok) $\quad$ [ stat ic ]}

Is the given string a valid query token.

Return true if it has the expected token format, otherwise false.

\subsubsection{4 long int ibis::query::limit (const char $*$ names, int direction, uint32_t $k e e p$, bool updateHits $=$ true)}

Truncate the bundles to provide the top-K rows of the bundles.

It returns the number of results kept, which is the smaller of the current number of bundles and the input argument keep. A negative value is returned in case of error, e.g., query has not been fully specified. If the second argument is true, the internal hit vector is updated to match the truncated solution. Otherwise, the internal hit vector is left unchanged. Since the functions getNumHits and getQualifiedXXX uses this internal hit vector, it is generally a good idea to update the hit vector. On the other hand, one may wish to avoid this update if the hit vector is not used in any way.

\subsubsection{5 void ibis::query::logMessage (const char $*$ event, const char $* f m t, \ldots)$ const}

Used to print information about the progress or state of query processing.

It prefixes each message with a query token.

\subsubsection{6 int ibis::query::orderby (const char $*$ names, int direction) const}

Re-order the bundles according the the new "ORDER BY" specification.

It returns 0 if it completes successfully. It returns a negative number to indicate error. If direction $>=0$, sort the values in ascending order, otherwise, sort them in descending order. 


\subsubsection{7 void ibis::query::orderPairs (const char $*$ p file) const $\quad$ [protected]}

Sort the content of the file as ibis: :rid_t.

It reads the content of the file one block at a time during the initial sorting of the blocks. It then merges the sorted blocks to produce a overall sorted file. Note that ibis : :rid_t is simply a pair of integers. Sinc the pairs are recorded as pairs of integers too, this should work.

\subsubsection{8 void ibis::query::printSelected (std::ostream \& out) const}

Print the values of the selected columns to the specified output stream.

The printed values are grouped by the columns without functions. For each group, the functions are evaluated on the columns named in the function. This is equivalent to have implicit "GROUP BY" and "ORDER BY" keywords on all columns appears without a function in the select clause.

\subsubsection{9 void ibis::query::printSelectedWithRID (std::ostream \& out) const}

Print the values of the columns in the select clause without functions.

One the groups of unique values are printed. For each group, the row ID (RID) of the rows are also printed.

\subsubsection{0 int64_t ibis::query::processJoin () [protected]}

Process the join operation and return the number of pairs.

Additionally, it performs only self-join, i.e., join a table with itself. This is only meant to test some algorithms for evaluating joins.

\subsubsection{1 std::string ibis::query::removeComplexConditions ()}

Separate out the sub-expressions that are not simple.

This is intended to allow the overall where clause to be evaluated in separated steps, where the simple conditions are left for this software to handle and the more complex ones are to be handled by another software. The set of conditions remain with this query object and the conditions returned by this function are assumed to be connected with the operator AND. If the top-most operator in the WHERE clause is not an AND operator, the whole clause will be returned if it contains any conditions that is not simple, otherwise, an empty string will be returned.

\subsubsection{2 ibis::bitvector $*$ ibis::query::sequentialScan () const}

Return a (new) bitvector that contains the result of directly scan the raw data to determine what records satisfy the user specified conditions.

It is mostly used for testing purposes. It can be called any time after the where clause is set, and does not change the state of the current query.

\subsubsection{3 int ibis::query::setSelectClause (const char $*$ str) [virtual]}

Specifies the select clause for the query.

The select clause is a string of attribute names (plus the four predefined functions, avg, max, min and sum) separated by spaces, commas (,) or semicolons(;). Repeated calls to this function simply overwrite the previous definition of the select clause. If no select clause is specified, the where clause alone determines whether record is a hit or not. The select clause will be reordered to make the plain column names without functions appear before with functions.

\subsubsection{4 int ibis::query::setTable (const ibis::part $* t b l$ )}

Resets the table used to evaluate the query conditions to the table specified in the argument. 
-1: nil pointer to table or empty table. -2: invalid string for select clause. -3 : select clause contains invalid column name. -4: invalid string for where clause. -5: where clause can not be parsed correctly. -6: where clause contains invalid column names or unsupported functions. -7: empty rid list for set rid operation. -8: neither rids nor range conditions are set. -9 : encountered some exceptional conditions during query evaluations. -10 : no private directory to store bundles. -11: Query not fully evaluated.

\subsubsection{5 int ibis::query::setWhereClause (const char $*$ str)}

Specifies the where clause for the query.

The where clause is of a string for a list of range conditions. A where clause is mandatory if a query is to be estimated or evaluated. This function may be called multiple times and each invocation will overwrite the previous where clause.

\subsubsection{6 int64_t ibis::query::sortEquiJoin (const ibis::rangeJoin \& cmp, const ibis::bitvector \& mask, const char $*$ pairfile const $\quad$ [protected]}

Perform equi-join by sorting the selected values.

This version reads the values marked to be 1 in the bitvector mask. It writes the the pairs satisfying the join condition to a file name pairfile.

\subsubsection{7 int64_t ibis::query::sortEquiJoin (const ibis::rangeJoin \& cmp, const ibis::bitvector \& mask) const} [protected]

Performing an equi-join by sorting the selected values first.

This version reads the values marked to be 1 in the bitvector mask and performs the actual operation of counting the number of pairs with equal values in memory.

3.83.3.28 int64_t ibis::query::sortRangeJoin (const ibis::rangeJoin \& cmp, const ibis::bitvector \& mask) const [protected]

Performing a range join by sorting the selected values.

The sorting is performed through std: : sort algorithm.

The documentation for this class was generated from the following files:

- query.h

- query.cpp

\subsection{4 ibis::query::result Class Reference}

The class ibis::query::result allows user to retrieve query result one row at a time.

\#include <bundle.h>

\section{Public Member Functions}

- uint32_t colPosition (const char $*$ cname) const

- double getDouble (uint32_t selind) const

Retrieve the value of column sel ind in the select clause as a double-precision floating-point number.

- double getDouble (const char $*$ cname) const

Retrieve the value of the named column as a double-precision floating-point number. 
- float getFloat (uint32_t selind) const

Retrieve the value of column selind in the select clause as a single-precision floating-point number.

- float getFloat (const char $*$ cname) const

Retrieve the value of the named column as a single-precision floating-point number.

- int getInt (uint32_t selind) const

Retrieve the value of column selind in the select clause as a signed integer.

- int getInt (const char $*$ cname) const

Retrieve the value of the named column as a signed integer.

- std::string getString (uint32_t selind) const

Retrieve the string value.

- std::string getString (const char $*$ cname) const

- unsigned getUInt (uint32_t selind) const

Retrieve the value of column selind in the select clause as an unsigned integer.

- unsigned getUInt (const char $*$ cname) const

Retrieve the value of the named column as an unsigned integer.

- bool next ()

- void reset ()

Move the internal pointer back to the beginning.

- result (ibis::query \&q)

\subsubsection{Detailed Description}

The class ibis::query::result allows user to retrieve query result one row at a time.

It matches the semantics of an ODBC cursor. That is the function next has to be called before the first set of results can be used.

\section{Note:}

This implementation stores the results in memory. Therefore, it is not suitable for handling large result sets.

\subsubsection{Member Function Documentation}

\subsubsection{1 int ibis::query::result::getInt (uint32_t selind) const [i in l ine ]}

Retrieve the value of column selind in the select clause as a signed integer.

Note:

Since this version avoids the name look up, it should be more efficient than the version taking the column name as argument.

\subsubsection{2 int ibis::query::result::getInt (const char $*$ cname) const}

Retrieve the value of the named column as a signed integer.

Note:

The name must appeared in the select clause of the query used to construct the result object. 


\subsubsection{3 std::string ibis::query::result::getString (uint32_t selind) const [i n l ine]}

Retrieve the string value.

\section{See also:}

ibis::bundle::getString for limitations.

\subsubsection{4 void ibis::query::result::reset ()}

Move the internal pointer back to the beginning.

Must call next to use the first set of results.

The documentation for this class was generated from the following files:

- bundle.h

- bundle.cpp

\subsection{5 ibis::range Class Reference}

The range encoded bitmap index based.

\#include <ibin.h>

Inheritance diagram for ibis::range::

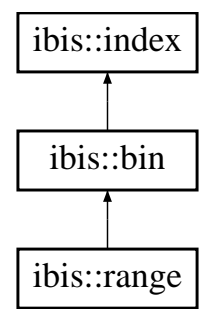

\section{Public Member Functions}

- long append (const ibis::range \&tail)

- virtual long append (const char $* \mathrm{dt}$, const char $* \mathrm{df}$, uint32_t nnew)

Extend the index.

- virtual void binBoundaries (std::vector $<$ double $>\&$ ) const

The function binBoundaries and binWeights return bin boundaries and counts of each bin respectively.

- virtual void binWeights (std::vector $<$ uint32_t $>\&$ ) const

- virtual int contractRange (ibis::qContinuousRange \&range) const

- virtual uint32_t estimate (const ibis::qContinuousRange \&expr) const

Returns an upper bound on the number of hits.

- virtual void estimate (const ibis::qContinuousRange \&expr, ibis::bitvector \&lower, ibis::bitvector \&upper) const

Computes an approximation of hits as a pair of lower and upper bounds.

- virtual long evaluate (const ibis::qContinuousRange \&expr, ibis::bitvector \&hits) const

To evaluate the exact hits. 
- virtual int expandRange (ibis::qContinuousRange \&range) const

The functions expandRange and contractRange expands or contracts the boundaries of a range condition so that the new range will have exact answers using the function estimate.

- virtual double getMax () const

The maximum value recorded in the index.

- virtual double getSum () const

Compute the approximate sum of all the values indexed.

- virtual const char $*$ name () const

Returns the name of the index, similar to the function type, but returns a string instead.

- virtual uint32_t numBins () const

- virtual void print (std::ostream \&out) const

Prints human readable information.

- range (const ibis::bin \&rhs)

- range (const ibis::column $*$ c, ibis:: fileManager::storage $*$ st, uint32_t offset=8)

- range (const ibis:: column $* \mathrm{c}=0$, const char $* \mathrm{f}=0$ )

- void read (int fdes, uint32_t offset, const char $*$ fname)

Read an ibis:: ragne embedded with multiple data structures.

- virtual void read (ibis::fileManager::storage $*$ st)

Reconstructs an index from an array of bytes.

- virtual void read (const char $*$ idxfile)

Reconstructs an index from the named file.

- virtual void speedTest (std::ostream \&out) const

Time some logical operations and print out their speed.

- virtual INDEX_TYPE type () const

Returns an index type identifier.

- virtual float undecidable (const ibis::qContinuousRange \&expr, ibis::bitvector \&iffy) const

Mark the position of the rows that can not be decided with this index.

- virtual void write (const char $* \mathrm{dt}$ ) const

Save index to a file.

\section{Protected Member Functions}

- virtual double computeSum () const

- virtual void locate (const ibis::qContinuousRange \&expr, uint32_t \&cand0, uint32_t \&cand1, uint32_t \&hit0, uint32_t \&hit1) const

- virtual void locate (const ibis::qContinuousRange \&expr, uint32_t \&cand0, uint32_t \&cand1) const

- virtual uint32_t locate (const double \&val) const 


\section{Protected Attributes}

- double max1

- double min1

\section{Friends}

- class ibis::pale

\subsubsection{Detailed Description}

The range encoded bitmap index based.

It can be thought of as a cumulative version of ibis::bin, where the ith bit vector marks the possibles of all entries where $\mathrm{x}<$ bounds [i].

\subsubsection{Member Function Documentation}

3.85.2.1 void ibis::range::estimate (const ibis::qContinuousRange \& expr, ibis::bitvector \& lower, ibis::bitvector \& upper) const [ virtual]

Computes an approximation of hits as a pair of lower and upper bounds.

\section{Parameters:}

expr the query expression to be evaluated.

lower a bitvector marking a subset of the hits. All rows marked with one (1) are definitely hits.

upper a bitvector marking a superset of the hits. All hits are marked with one, but some of the rows marked one may not be hits. If the variable upper is empty, the variable lower is assumed to contain the exact answer.

Reimplemented from ibis::bin.

3.85.2.2 long ibis::range::evaluate (const ibis::qContinuousRange \& expr, ibis::bitvector \& hits) const [virtual]

To evaluate the exact hits.

On success, return the number of hits, otherwise a negative value is returned.

Reimplemented from ibis::bin.

\subsubsection{3 int ibis::range::expandRange (ibis::qContinuousRange \& range) const [virtua l]}

The functions expandRange and contractRange expands or contracts the boundaries of a range condition so that the new range will have exact answers using the function estimate.

The default implementation provided does nothing since this is only meaningful for indices based on bins.

Reimplemented from ibis::bin.

\subsubsection{4 double ibis::range::getSum () const [virtual]}

Compute the approximate sum of all the values indexed.

If it decides that computing the sum directly from the vertical partition is more efficient, it will return NaN immediately. Reimplemented from ibis::bin. 


\subsubsection{5 void ibis::range::print (std::ostream \& out) const [ virtual]}

Prints human readable information.

Outputs information about the index as text to the specified output stream.

Reimplemented from ibis::bin.

\subsubsection{6 void ibis::range::read (ibis::fileManager::storage $* s t$ ) [virtual]}

Reconstructs an index from an array of bytes.

Intended for internal use only!

Reimplemented from ibis::bin.

\subsubsection{7 void ibis::range::read (const char $*$ idxfile) $\quad$ [virtual]}

Reconstructs an index from the named file.

The name can be the directory containing an index file. In this case, the name of the index file must be the name of the column followed by ".idx" suffix.

Reimplemented from ibis::bin.

3.85.2.8 float ibis::range::undecidable (const ibis::qContinuousRange \& expr, ibis::bitvector \& iffy) const [virtual]

Mark the position of the rows that can not be decided with this index.

\section{Parameters:}

expr the range conditions to be evaluated.

iffy the bitvector marking the positions of rows that can not be decided using the index. Return value is the expected fraction of undecided rows that might satisfy the range conditions.

Reimplemented from ibis::bin.

\subsubsection{9 void ibis::range::write (const char $* \boldsymbol{d t}$ ) const [virtual]}

Save index to a file.

Outputs the index in a compact binary format to the named file or directory. The index file contains a header that can be identified by the function isIndex.

Reimplemented from ibis::bin.

The documentation for this class was generated from the following files:

- ibin.h

- irange.cpp

\subsection{6 ibis::rangeJoin Class Reference}

A join is defined by two names and a numerical expression.

\#include <qExpr.h>

Inheritance diagram for ibis::rangeJoin:: 


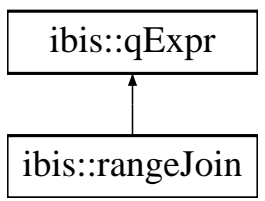

\section{Public Member Functions}

- virtual rangeJoin $*$ dup () const

- const char $*$ getName1 () const

- const char $*$ getName2 () const

- const ibis::compRange::term $*$ getRange () const

- ibis::compRange::term $*$ getRange ()

- virtual void print (std::ostream \&out) const

Print out the node in the string form.

- rangeJoin (const char $* \mathrm{n} 1$, const char $* \mathrm{n} 2$, ibis::compRange::term $* \mathrm{x}$ )

- rangeJoin (const char $* \mathrm{n} 1$, const char $* \mathrm{n} 2$ )

- void setRange (ibis::compRange::term $* \mathrm{t}$ )

\subsubsection{Detailed Description}

A join is defined by two names and a numerical expression.

If the numerical expression is not specified, it is a standard equal-join, 'name $1=$ name2'. If the numerical expression is specified, it is a range-join, 'name1 between name 2 - expr and name $2+$ expr'.

The documentation for this class was generated from the following files:

- qExpr.h

- qExpr.cpp

\subsection{7 ibis::relic Class Reference}

The basic bitmap index.

\#include <irelic.h>

Inheritance diagram for ibis::relic::

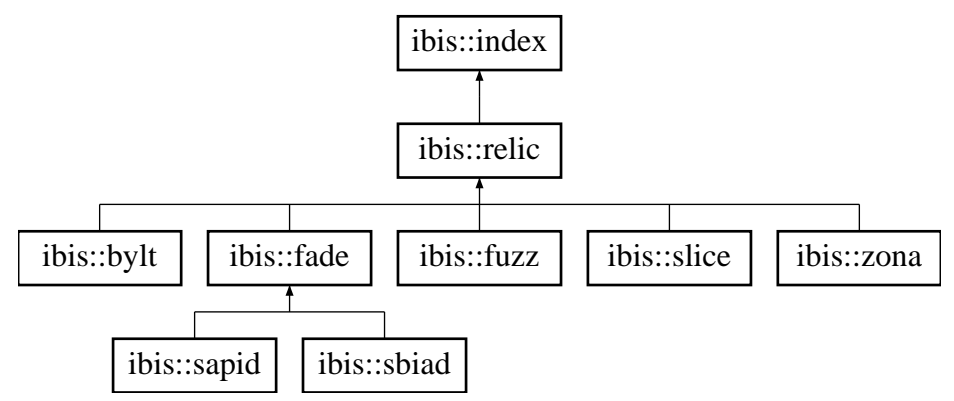

\section{Public Member Functions}

- long append (const array_t $<$ uint32_t $>$ \&ind) 
Append a list of integers.

- long append (const ibis::relic \&tail)

- virtual long append (const char $* \mathrm{dt}$, const char $* \mathrm{df}$, uint32_t nnew)

Extend the index.

- virtual void binBoundaries (std::vector $<$ double $>\&$ b) const

The function binBoundaries and binWeights return bin boundaries and counts of each bin respectively.

- virtual void binWeights (std::vector $<$ uint32_t $>\& b)$ const

- virtual int64_t estimate (const ibis::relic \&idx2, const ibis::rangeJoin \&expr, const ibis::bitvector \&mask, const ibis::qRange $*$ const range1, const ibis::qRange $*$ const range2) const

- virtual int64_t estimate (const ibis::relic \&idx2, const ibis::rangeJoin \&expr, const ibis::bitvector \&mask) const

Estimate an upper bound for the number of pairs produced from marked records.

- virtual void estimate (const ibis::relic \&idx2, const ibis::rangeJoin \&expr, const ibis::bitvector \&mask, const ibis::qRange $*$ const range1, const ibis::qRange $*$ const range2, ibis::bitvector64 \&lower, ibis::bitvector64 \&upper) const

Note:

It is assumed that range 1 is for column 1 in the join expression and range 2 is for column 2 in the join expression.

- virtual void estimate (const ibis::relic \&idx2, const ibis::rangeJoin \&expr, const ibis::bitvector \&mask, ibis::bitvector64 \&lower, ibis::bitvector64 \&upper) const

Estimate the pairs for the range join operator.

- virtual uint32_t estimate (const ibis::qDiscreteRange \&expr) const

- virtual void estimate (const ibis::qDiscreteRange \&expr, ibis::bitvector \&lower, ibis::bitvector \&upper) const

Estimate the hits for discrete ranges, i.e., those translated from 'a IN $(x, y$, .

- virtual uint32_t estimate (const ibis::qContinuousRange \&expr) const

Returns an upper bound on the number of hits.

- virtual void estimate (const ibis::qContinuousRange \&expr, ibis::bitvector \&lower, ibis::bitvector \&upper) const

Computes an approximation of hits as a pair of lower and upper bounds.

- virtual double estimateCost (const ibis::qDiscreteRange \&expr) const

- virtual double estimateCost (const ibis::qContinuousRange \&expr) const

Estimate the code of evaluate a range condition.

- virtual long evaluate (const ibis::qDiscreteRange \&expr, ibis::bitvector \&hits) const

- virtual long evaluate (const ibis::qContinuousRange \&expr, ibis::bitvector \&hits) const

To evaluate the exact hits.

- virtual long getCumulativeDistribution (std::vector $<$ double $>$ \&bds, std::vector $<$ uint32_t $>$ \&cts) const

Cumulative distribution of the data.

- virtual long getDistribution (std::vector $<$ double $>$ \&bds, std::vector $<$ uint32_t $>$ \&cts) const

Binned distribution of the data.

- virtual double getMax () const

The maximum value recorded in the index. 
- virtual double getMin () const

The minimum value recorded in the index.

- virtual double getSum () const

Compute the approximate sum of all the values indexed.

- array_t $<$ uint32_t $>*$ keys (const ibis::bitvector \&mask) const

- virtual const char $*$ name () const

Returns the name of the index, similar to the function type, but returns a string instead.

- virtual void print (std::ostream \&out) const

Prints human readable information.

- virtual void read (ibis::fileManager::storage $*$ st)

Reconstructs an index from an array of bytes.

- virtual void read (const char $*$ idxfile)

Reconstructs an index from the named file.

- relic (const ibis::column $*$ c, ibis::fileManager::storage $*$ st, uint32_t offset=8)

The content of the file (following the 8-byte header) is nrows(uint32_t) - number of bits in each bit sequences nobs (uint32_t) - number of bit sequences card (uint32_t) - the number of distinct values, i.e., cardinality (padding to ensure the next data element is on 8-byte boundary) values (double[card]) - the values as doubles offset (uint32_t[nobs +1$])$ the starting positions of the bit sequences (as bit vectors) bitvectors - the bitvectors one after another.

- relic (const ibis::column $* \mathrm{c}$, uint32_t card, array_t $<$ uint32_t $>$ \&ints)

Construct an index from an integer array.

- relic (const ibis::column $*$ c, uint32_t popu, uint32_t ntpl=0)

Construct a dummy index.

- relic (const ibis::column $* \mathrm{c}$, const char $* \mathrm{f}=0$ )

- virtual void speedTest (std::ostream \&out) const

Time some logical operations and print out their speed.

- virtual INDEX_TYPE type () const

Returns an index type identifier.

- virtual float undecidable (const ibis::qDiscreteRange \&expr, ibis::bitvector \&iffy) const

- virtual float undecidable (const ibis::qContinuousRange \&expr, ibis::bitvector \&iffy) const

This class and its derived classes should produce exact answers, therefore no undecidable rows.

- virtual void write (const char $* \mathrm{dt})$ const

Save index to a file.

\section{Protected Member Functions}

- virtual void clear ()

Clear the existing content.

- virtual double computeSum () const 
- template $<$ typename $\mathrm{E}>$ void construct (const array_t $<\mathrm{E}>$ \&arr)

Construct an index from in-memory values.

- void locate (const ibis::qContinuousRange \&expr, uint32_t \&hit0, uint32_t \&hit1) const

- uint32_t locate (const double \&val) const

- void write (int fdes) const

Protected Attributes

- array_t $\mathrm{t}<$ double $>$ vals

\subsubsection{Detailed Description}

The basic bitmap index.

It generates one bitmap for each distinct value.

\subsubsection{Constructor \& Destructor Documentation}

3.87.2.1 ibis::relic::relic (const ibis::column $* \boldsymbol{c}$, uint32_t popu, uint32_t $n t p l=0$ )

Construct a dummy index.

All entries have the same value popu. This is used to generate index for meta tags from STAR data.

\subsubsection{Member Function Documentation}

\subsubsection{1 long ibis::relic::append (const array_t $<$ uint32_t $>$ \& ind)}

Append a list of integers.

The integers are treated as bin numbers. This function is primarily used by ibis::category::append().

\subsubsection{2 template $<$ typename $\mathbf{E}>$ void ibis::relic::construct (const array_t $<\mathbf{E}>\boldsymbol{\&}$ arr) $\quad$ [protected]}

Construct an index from in-memory values.

The type $\mathrm{E}$ is intended to be element types supported in column.h.

3.87.3.3 void ibis::relic::estimate (const ibis::relic \& $i d x 2$, const ibis::rangeJoin \& expr, const ibis::bitvector \& mask, const ibis::qRange $*$ const range1, const ibis::qRange $*$ const range2, ibis::bitvector64 \& lower, ibis::bitvector64 \& upper) const [virtual]

Note:

It is assumed that range 1 is for column 1 in the join expression and range 2 is for column 2 in the join expression.

No name matching is performed.

3.87.3.4 void ibis::relic::estimate (const ibis::relic \& $i d x 2$, const ibis::rangeJoin \& expr, const ibis::bitvector \& mask, ibis::bitvector64 \& lower, ibis::bitvector64 \& upper) const [virtual]

Estimate the pairs for the range join operator.

Only records that are masked are evaluated. 
3.87.3.5 virtual void ibis::relic::estimate (const ibis::qDiscreteRange \& expr, ibis::bitvector \& lower, ibis::bitvector \& upper) const [inline, virtual]

Estimate the hits for discrete ranges, i.e., those translated from 'a IN (x, y, .

.)'.

Reimplemented from ibis::index.

3.87.3.6 virtual void ibis::relic::estimate (const ibis::qContinuousRange \& expr, ibis::bitvector \& lower, ibis::bitvector \& upper) const [inline, virtual]

Computes an approximation of hits as a pair of lower and upper bounds.

\section{Parameters:}

expr the query expression to be evaluated.

lower a bitvector marking a subset of the hits. All rows marked with one (1) are definitely hits.

upper a bitvector marking a superset of the hits. All hits are marked with one, but some of the rows marked one may not be hits. If the variable upper is empty, the variable lower is assumed to contain the exact answer.

Implements ibis::index.

Reimplemented in ibis::slice.

3.87.3.7 long ibis::relic::evaluate (const ibis::qContinuousRange \& expr, ibis::bitvector \& hits) const [virtual]

To evaluate the exact hits.

On success, return the number of hits, otherwise a negative value is returned.

Implements ibis::index.

Reimplemented in ibis::slice, ibis::fade, ibis::sbiad, ibis::sapid, ibis::fuzz, ibis::bylt, and ibis::zona.

\subsubsection{8 double ibis::relic::getSum () const [virtual]}

Compute the approximate sum of all the values indexed.

If it decides that computing the sum directly from the vertical partition is more efficient, it will return NaN immediately. Implements ibis::index.

Reimplemented in ibis::slice, and ibis::fade.

\subsubsection{9 void ibis::relic::print (std::ostream \& out) const [ virtua l]}

Prints human readable information.

Outputs information about the index as text to the specified output stream.

Implements ibis::index.

Reimplemented in ibis::slice, ibis::fade, ibis::sbiad, ibis::sapid, ibis::fuzz, ibis::bylt, and ibis::zona.

\subsubsection{0 void ibis::relic::read (ibis::fileManager::storage $* s t$ ) [virtual]}

Reconstructs an index from an array of bytes.

Intended for internal use only!

Implements ibis::index.

Reimplemented in ibis::slice, ibis::fade, ibis::fuzz, ibis::bylt, and ibis::zona. 


\subsubsection{1 void ibis::relic::read (const char $*$ idxfile) $\quad$ [virtual]}

Reconstructs an index from the named file.

The name can be the directory containing an index file. In this case, the name of the index file must be the name of the column followed by ".idx" suffix.

Implements ibis::index.

Reimplemented in ibis::slice, ibis::fade, ibis::fuzz, ibis::bylt, and ibis::zona.

\subsubsection{2 void ibis::relic::write (const char $* \boldsymbol{d} t)$ const [virtual]}

Save index to a file.

Outputs the index in a compact binary format to the named file or directory. The index file contains a header that can be identified by the function isIndex.

Implements ibis::index.

Reimplemented in ibis::slice, ibis::fade, ibis::sbiad, ibis::sapid, ibis::fuzz, ibis::bylt, and ibis::zona.

The documentation for this class was generated from the following files:

- irelic.h

- irelic.cpp

\subsection{8 ibis::resource Class Reference}

A container for name-value pairs.

\#include <resource.h>

\section{Public Types}

- typedef $\operatorname{std::map}<$ const char $*$, resource $*$, ibis::lessi $>$ gList

The name-value pairs are categorized into two types, names that map to simple values (vList) and names that map to groups of name-value pairs ( $\mathrm{gList}$ ).

- typedef std::map $<$ const char $*$, char $*$, ibis::lessi $>$ vList

\section{Public Member Functions}

- void add (const char $*$ name, const char $*$ value)

Insert a new name-value pair.

- bool empty () const

Returns true if there is no name-value pair on record.

- gList::const_iterator gBegin () const

- gList::const_iterator gEnd () const

- const resource $*$ getGroup (const char $*$ name) const

Find a group with the given name.

- double getNumber (const char *name) const

Parse the string value as a number.

- std::string getPrefix () const 
Return the name of the full prefix of the resource.

- const char $*$ getValue (const char $*$ name) const

Find a named parameter.

- bool isTrue (const char $*$ name) const

If the named parameter exists and its value is one of "true", "yes", "on" or "1", this function will return true, otherwise false.

- const resource \& operator= (const resource \&rhs)

The assignment operator.

- const char $*$ operator[ ] (const char $*$ name) const

This group of functions search through multiple levels of the name hierarchy.

- void read (const char $* \mathrm{fn}=0$ )

Read the content of the file and add it to the existing lists of name-value pairs.

- resource (const resource \&rhs)

- resource (const resource $*$ ctx, const char $* \mathrm{pfx}$ )

- resource (const char $* \mathrm{fn}=0$ )

- vList::const_iterator vBegin () const

- vList::const_iterator vEnd () const

- void write (const char $* \mathrm{fn}=0$ ) const

Write the name-value pairs to the named file.

\section{Static Public Member Functions}

- static void clear (vList \&vl)

Clear a $v$ List.

- static bool isStringTrue (const char *val)

Returns true is the string value should be interpreted as logical truth.

- static void parseNameValuePairs (const char $*$ in, vList \&lst)

Parse a string into a name-value list.

\subsubsection{Detailed Description}

A container for name-value pairs.

It is mostly used for storing the configuration parameters. The parameters are in a format as follows: group:group:...:name=value where the delimiter can be either ' $*$ ', '.' or '.' and anything following the first '=' sign is assumed to be part of the value string until the end of line. The leading and trailing spaces are removed from both the name and the value. The specification that appears later in the same configuration file or read later (through a call to read()) will overwrite the parameter with the same name. This include the groups and individual parameters. For example if a parameter named 'abcd' is specified first, but later, the same name is used as a group name, then the previously specified parameter will be removed from the list and the new group will be inserted. If the parameter with name 'abcd' appeared again, then the group 'abcd' will be removed and the named parameter will be inserted.

The line length must of no more than MAX_LINE defined in const.h.

The top level group name can be any one of the following: all, common, and ' $*$ '. When writing out the parameters, the top level name is not written. 


\subsubsection{Member Function Documentation}

\subsubsection{1 void ibis::resource::add (const char $*$ name, const char $*$ value)}

Insert a new name-value pair.

It replaces the existing value.

\subsubsection{2 const ibis::resource $*$ ibis::resource::getGroup (const char $*$ name) const [in line ]}

Find a group with the given name.

The name is expected to be a simple name without any separators. Any separator in the name will cause it to return a nil pointer.

\subsubsection{3 double ibis::resource::getNumber (const char $*$ name) const}

Parse the string value as a number.

If the first non-numeric character is a ' $\mathrm{k}$ ' or ' $\mathrm{m}$ ' or ' $\mathrm{g}$ ', the proceeding number is mutiplied by 1024,1048576 , or 1073742824. If the first non-numeric character is ' $h$ ', the value before it is multiplied by 3600 (h for hour), converting it from hours to seconds.

\subsubsection{4 const char $*$ ibis::resource::getValue (const char $*$ name) const [inline ]}

Find a named parameter.

The name is expected to be a simple name without any separators. Any separator in it will cause a nil pointer to be returned.

3.88.2.5 ] const char $*$ ibis::resource::operator[ ] (const char $*$ name) const

This group of functions search through multiple levels of the name hierarchy.

The operator[] returns a pointer to the string value, get Number returns the string value as a number, and is True returns the string value as boolean.

The incoming name can contain multiple separators. Each component of the name is separated by one separator. From the left to right, the left-most component defines the highest level of the hierarchy. A high-level name forms the context for the next level of the name hierarchy. The final component of the name is directly associated with a string value. The search algorithm first descend to the lowest level with the matching names and starts to look for a name that matches the last component of the specified name. If a match is not found, it will go back one level and perform the same search. This continues until a match is found or it has searched all the levels.

\subsubsection{6 void ibis::resource::parseNameValuePairs (const char $*$ in, vList \& lst) [ stat ic ]}

Parse a string into a name-value list.

Add the new ones to the existing list.

\subsubsection{7 void ibis::resource::read (const char $* \boldsymbol{f} \boldsymbol{n}=0$ )}

Read the content of the file and add it to the existing lists of name-value pairs.

It will read the first file in the following list and add the content to the existing list of parameter,

- (1) argument to this function (fn),

- (2) environment variable IBISRC,

- (3) file named ibis.rc in the current directory, 
- (4) file named .ibisrc in the current directory,

- (5) file named .ibisrc in the user's home directory.

It will attempt to parse the content of the first file it finds. The content of the file is parsed and added to the current content of the resouce object. The parameters with the same will overwrite the existing values. If it can not find any one of the files, it will return without modifying the current content of the resource object.

\subsubsection{8 void ibis::resource::write (const char $* \boldsymbol{f n}=0$ ) const}

Write the name-value pairs to the named file.

If the file name is a nil pointer, the pairs are written to the standard output. If it can not open the named file, it will also write to the standard output.

The documentation for this class was generated from the following files:

- resource.h

- resource.cpp

\subsection{9 ibis::rid_t Union Reference}

The object identifiers used to distinguish records.

\#include <const.h>

\section{Public Member Functions}

- bool operator!= (const rid_t \&r) const

- bool operator $<$ (const rid_t \& r) const

- bool operator $<=$ (const rid_t \&r) const

- bool operator $==$ (const rid_t $\& r$ ) const

- bool operator $>$ (const rid_t \& r) const

- bool operator $>=$ (const rid_t \&r) const

\section{Public Attributes}

- ibis::rid_t::name num

As two 32-bit values.

- uint64_t value

As a single 64-bit value.

\section{Classes}

- struct name

As two 32-bit values.

\subsubsection{Detailed Description}

The object identifiers used to distinguish records.

The documentation for this union was generated from the following file:

- const.h 


\subsection{0 ibis::rid_t::name Struct Reference}

As two 32-bit values.

\#include <const.h>

\section{Public Attributes}

- uint32_t event

Event number. Less significant.

- uint32_t run

Run number. More significant.

\subsubsection{Detailed Description}

As two 32-bit values.

The documentation for this struct was generated from the following file:

- const.h

\subsection{1 ibis::ridHandler Class Reference}

A class for handling file IO for ibis::rid_t.

\#include <rids.h>

\section{Public Member Functions}

- int append (const RIDSet \&rids, const char $*$ destination) const

Append the rid set to the name file.

- int read (RIDSet \&rids, const char $*$ source)

This function is capable of reading a file written with one write command and multiple append commands.

- ridHandler (const char $*$ dbName, const char $*$ pref="ibis")

- int write (const RIDSet \&rids, const char $*$ destination, const char $* \mathrm{dbName}=0$ )

Write the rid set.

\section{Protected Member Functions}

- int matchDBName (std::istream \&_from) const

- int readDBName (std::istream \&_from)

- int readRidCount (std::istream \&_from, int \&ic) const

- int readVersion (std::istream \&_from) const

\section{Protected Attributes}

- char *_dbName

- char *_prefix

- pthread_mutex_t mutex 


\section{Static Protected Attributes}

- static const char $*$ const version

Implements the functions defined in ibis::ridHandler.

\subsubsection{Detailed Description}

A class for handling file IO for ibis::rid_t.

\subsubsection{Member Function Documentation}

\subsubsection{1 int ibis::ridHandler::append (const RIDSet \& rids, const char $*$ fname) const}

Append the rid set to the name file.

Return the number of rids written. This function can be called after ibis::ridHandler::write has been called to write a file. It can be called many times. The function ibis::ridHandler::read will concatenate all rid sets into one.

\subsubsection{2 int ibis::ridHandler::read (ibis::RIDSet \& rids, const char $*$ fname)}

This function is capable of reading a file written with one write command and multiple append commands.

All rids are placed in

- rids in the order they appear in the file. The member varialbe

- _dbName will be set to the name stored in the file.

\subsubsection{3 int ibis::ridHandler::write (const RIDSet \& rids, const char $*$ fname, const char $* d b N a m e=0$ )}

Write the rid set.

Return the number of rids written. If the first argument is specified, the internally stored dbName would be modified. The documentation for this class was generated from the following files:

- rids.h

- rids.cpp

\subsection{2 ibis::roster Class Reference}

A roster list is a list of indices for ordering the values in the ascending order.

\#include <iroster.h>

\section{Public Member Functions}

- const array_t $\mathrm{t}<$ uint32_t $>\&$ array () const

- const ibis::column $*$ getColumn () const

- template $<$ typename T $>$ void icSearch (const std::vector $<\mathrm{T}>$ \&vals, std::vector $<$ uint32_t $>$ \&pos) const

- int locate (const std::vector $<$ double $>\&$ vals, ibis::bitvector \&positions) const

- int locate (const std::vector $<$ float $>\&$ vals, ibis::bitvector \&positions) const

- int locate (const std::vector $<$ uint32_t $>$ \&vals, ibis::bitvector \&positions) const

- int locate (const std::vector $<$ int32_t $>$ \&vals, ibis::bitvector \&positions) const 
- const char $*$ name () const

- template $<$ typename $\mathrm{T}>$ void oocSearch (const std::vector $<\mathrm{T}>$ \&vals, std::vector $<$ uint32_t $>$ \&pos) const

- uint32_t operator[] (uint32_t i) const

- void print (std::ostream \&out) const

Output minimal information about the roster list.

- void read (ibis::fileManager::storage $*$ st)

- void read (const char $*$ idxfile)

- roster (const ibis::column $* \mathrm{c}$, const ibis::bitvector \&mask, const char $* \mathrm{f}=0$ )

Select the values that are marked 1 in mask and sort them.

- roster (const ibis::column $* \mathrm{c}$, ibis::fileManager::storage $*$ st, uint32_t offset=8)

For reconstructing the data structure from raw bytes stored in st.

- $\operatorname{roster}($ const ibis::column $* \mathrm{c}$, const char $* \mathrm{f}=0$ )

Sort all data elements in the named file (default to the one in the current working directory).

- uint32_t size () const

- void write (const char $* \mathrm{dt})$ const

Write two files, ind for indices and srt to the sorted values.

- void writeSorted (const char $* \mathrm{dt}$ ) const

Write the sorted version of the attribute values to a srt file.

\section{Static Public Member Functions}

- template $<$ class $\mathrm{T}>$ static long mergeBlock2 (const char $*$ dsrc, const char $*$ dout, const uint32_t segment, array_$\mathrm{t}<\mathrm{T}>$ \&buf1, array_t $<\mathrm{T}>$ \&buf2, array_t $<\mathrm{T}>$ \&buf3)

A two-way merge algorithm.

\subsubsection{Detailed Description}

A roster list is a list of indices for ordering the values in the ascending order.

It can use external sort if the data and indices can not fit into memory. The indices will be written to a file with .ind extension and if the external sorting procedure is used a file with .srt extension is also generated to store a sorted version of the values. If the indices can not be loaded into memory as a whole, the .ind file will be opened for future operations.

\subsubsection{Constructor \& Destructor Documentation}

\subsubsection{1 ibis::roster::roster (const ibis::column $* c$, ibis::fileManager::storage $*$ st, uint32_t offset $=8$ )}

For reconstructing the data structure from raw bytes stored in st.

The content of the file (following the 8-byte header) is the index array ind. 


\subsubsection{Member Function Documentation}

\subsubsection{1 int ibis::roster::locate (const std::vector $<$ int32_t $>\&$ vals, ibis::bitvector \& positions) const}

Locate the the values and set their positions in the bitvector.

Return a negative value for error, zero or a positive value for in case of success. The input values are assumed to be sorted in ascending order.

3.92.3.2 template $<$ class $\mathbf{T}>$ long ibis::roster::mergeBlock2 (const char $*$ dsrc, const char $*$ dout, const uint32_t segment, array_t $<\mathbf{T}>\boldsymbol{\&}$ buf1, array_t $<\mathbf{T}>\boldsymbol{\&}$ buf2, array_t $<\mathbf{T}>\boldsymbol{\&}$ buf3) $\quad$ [static ]

A two-way merge algorithm.

Uses std::less $<\mathrm{T}>$ for comparisons. Assumes the sorted segment size is segment elements of type $\mathrm{T}$.

\subsubsection{3 void ibis::roster::writeSorted (const char $* d f$ ) const}

Write the sorted version of the attribute values to a .srt file.

Attempt to read the whole column into memory first. If it fails to do so, it will read one value at a time from the original data file.

The documentation for this class was generated from the following files:

- iroster.h

- iroster.cpp

\subsection{3 ibis::sapid Class Reference}

The multicomponent equality encoded index.

\#include <irelic.h>

Inheritance diagram for ibis::sapid::

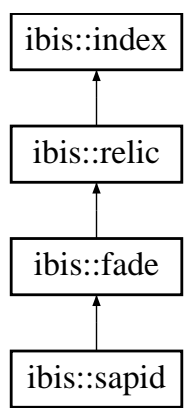

\section{Public Member Functions}

- virtual long append (const char $* \mathrm{dt}$, const char $* \mathrm{df}$, uint32_t nnew)

Extend the index.

- virtual long evaluate (const ibis::qDiscreteRange \&expr, ibis::bitvector \&hits) const

- virtual long evaluate (const ibis::qContinuousRange \&expr, ibis::bitvector \&hits) const

To evaluate the exact hits.

- virtual const char $*$ name () const 
Returns the name of the index, similar to the function type, but returns a string instead.

- virtual void print (std::ostream \&out) const

Prints human readable information.

- sapid (const ibis::column $* \mathrm{c}$, ibis::fileManager::storage $*$ st, uint32_t offset=8)

- sapid (const ibis::column $* \mathrm{c}=0$, const char $* \mathrm{f}=0$, const uint32_t nbase $=2$ )

- virtual void speedTest (std::ostream \&out) const

Time some logical operations and print out their speed.

- virtual INDEX_TYPE type () const

Returns an index type identifier.

- virtual void write (const char $* \mathrm{dt}$ ) const

Save index to a file.

\subsubsection{Detailed Description}

The multicomponent equality encoded index.

\subsubsection{Member Function Documentation}

3.93.2.1 long ibis::sapid::evaluate (const ibis::qContinuousRange \& expr, ibis::bitvector \& hits) const [virtual]

To evaluate the exact hits.

On success, return the number of hits, otherwise a negative value is returned.

Reimplemented from ibis::fade.

\subsubsection{2 void ibis::sapid::print (std::ostream \& out) const [virtual]}

Prints human readable information.

Outputs information about the index as text to the specified output stream.

Reimplemented from ibis::fade.

\subsubsection{3 void ibis::sapid::write (const char $* \boldsymbol{d t}$ ) const [ virtual]}

Save index to a file.

Outputs the index in a compact binary format to the named file or directory. The index file contains a header that can be identified by the function isIndex.

Reimplemented from ibis::fade.

The documentation for this class was generated from the following files:

- irelic.h

- isapid.cpp

\subsection{4 ibis::sbiad Class Reference}

The multicomponent interval encoded index. 
\#include <irelic.h>

Inheritance diagram for ibis::sbiad::

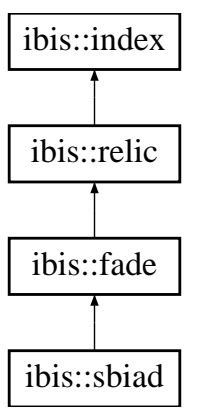

\section{Public Member Functions}

- virtual long append (const char $* \mathrm{dt}$, const char $* \mathrm{df}$, uint32_t nnew)

Extend the index.

- virtual long evaluate (const ibis::qDiscreteRange \&expr, ibis::bitvector \&hits) const

- virtual long evaluate (const ibis::qContinuousRange \&expr, ibis::bitvector \&hits) const To evaluate the exact hits.

- virtual const char $*$ name () const

Returns the name of the index, similar to the function type, but returns a string instead.

- virtual void print (std::ostream \&out) const

Prints human readable information.

- sbiad (const ibis::column $*$ c, ibis::fileManager::storage $*$ st, uint32_t offset=8)

- sbiad (const ibis: :column $* \mathrm{c}=0$, const char $* \mathrm{f}=0$, const uint32_t nbase $=2$ )

- virtual void speedTest (std::ostream \&out) const

Time some logical operations and print out their speed.

- virtual INDEX_TYPE type () const

Returns an index type identifier.

- virtual void write (const char $* \mathrm{dt}$ ) const

Save index to a file.

\subsubsection{Detailed Description}

The multicomponent interval encoded index.

Defined by Chan and Ioannidis (SIGMOD 99).

\subsubsection{Member Function Documentation}

3.94.2.1 long ibis::sbiad::evaluate (const ibis::qContinuousRange \& expr, ibis::bitvector \& hits) const [virtual]

To evaluate the exact hits. 
On success, return the number of hits, otherwise a negative value is returned.

Reimplemented from ibis::fade.

\subsubsection{2 void ibis::sbiad::print (std::ostream \& out) const [virtual]}

Prints human readable information.

Outputs information about the index as text to the specified output stream.

Reimplemented from ibis::fade.

\subsubsection{3 void ibis::sbiad::write (const char $* \boldsymbol{d t})$ const [ virtual]}

Save index to a file.

Outputs the index in a compact binary format to the named file or directory. The index file contains a header that can be identified by the function isIndex.

Reimplemented from ibis::fade.

The documentation for this class was generated from the following files:

- irelic.h

- isbiad.cpp

\subsection{5 ibis::selected Class Reference}

A data structure to store the select clause of a query.

\#include <util.h>

\section{Public Types}

- typedef std::vector $<$ std::string $>:$ :const_iterator const_iterator An iterator through the column names of the select clause.

- enum FUNCTION \{

NIL, AVG, MAX, MIN,

SUM \}

\section{Public Member Functions}

- const_iterator begin () const

- void clear ()

- bool empty () const

- const_iterator end () const

- size_t find (const char $*$ key) const Return the first occurrence of the string.

- FUNCTION getFunction (size_t i) const

- const char $*$ getName (size_t i) const

Access the ith column name of the select clause.

- const std::vector $<$ std::string $>\&$ getNames () const

Return the list of names stored internally. 
- std::string getTerm (size_t i) const

Return the ith term, with the function name.

- size_t nPlain () const

- const char $*$ operator $*()$ const

Output a stringlized version of the select clause.

- const char $*$ operator[ ] (size_t i) const

- void remove (const std::vector $<$ size_t $>$ \&ents)

Remove the entries specified.

- void select (const std::vector $<$ const char $*>\&$ nl, bool sort=true)

- void select (const char $*$ str, bool sort=true)

Parse the select clause. By default, place the functions last.

- selected (const char $*$ str)

The default constructor leaves data members empty.

- size_t size () const

\subsubsection{Detailed Description}

A data structure to store the select clause of a query.

A select clause may contain a list of names plus a list of simple functions. The supported functions are avg, max, min and sum. Each of these functions can only take a single name as it argument.

\subsubsection{Member Function Documentation}

\subsubsection{1 size_t ibis::selected::find (const char $*$ key) const}

Return the first occurrence of the string.

Returns the value of size if the given key in not in the list of selected components.

\subsubsection{2 const char $*$ ibis::selected::getName (size_t $i)$ const [i n l ine ]}

Access the ith column name of the select clause.

In case of a function, it returns the name of the argument rather than the whole function. This operator is only intended to be used to extract the column values.

The documentation for this class was generated from the following files:

- util.h

- query.cpp

\subsection{6 ibis::slice Class Reference}

The bit-sliced index (O'Neil). It used the binary encoding.

\#include <irelic.h>

Inheritance diagram for ibis::slice:: 


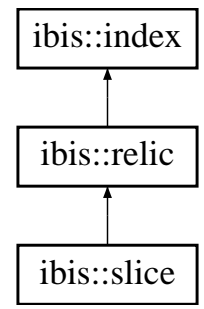

\section{Public Member Functions}

- virtual long append (const char $* \mathrm{dt}$, const char $* \mathrm{df}$, uint32_t nnew)

Extend the index.

- virtual void binWeights (std::vector $<$ uint32_t $>\& b$ ) const

- virtual uint32_t estimate (const ibis::qContinuousRange \&expr) const

Returns an upper bound on the number of hits.

- virtual void estimate (const ibis::qContinuousRange \&expr, ibis::bitvector \&lower, ibis::bitvector \&upper) const

Computes an approximation of hits as a pair of lower and upper bounds.

- virtual double estimateCost (const ibis::qDiscreteRange \&expr) const

- virtual double estimateCost (const ibis::qContinuousRange \&expr) const

Estimate the code of evaluate a range condition.

- virtual long evaluate (const ibis::qDiscreteRange \&expr, ibis::bitvector \&hits) const

- virtual long evaluate (const ibis::qContinuousRange \&expr, ibis::bitvector \&hits) const

To evaluate the exact hits.

- virtual double getSum () const

Compute the approximate sum of all the values indexed.

- virtual const char $*$ name () const

Returns the name of the index, similar to the function type, but returns a string instead.

- virtual void print (std::ostream \&out) const

Prints human readable information.

- virtual void read (ibis::fileManager::storage $*$ st)

Reconstruct an index from a piece of consecutive memory.

- virtual void read (const char $*$ idxfile)

Read the index contained in the file $f$.

- slice (const ibis::column $*$ c, ibis::fileManager::storage $*$ st, uint32_t offset=8)

- slice (const ibis:: column $* \mathrm{c}=0$, const char $* \mathrm{f}=0$ )

- virtual void speedTest (std::ostream \&out) const

Time some logical operations and print out their speed.

- virtual INDEX_TYPE type () const

Returns an index type identifier. 
- virtual void write (const char $* \mathrm{dt}$ ) const Save index to a file.

\section{Protected Member Functions}

- virtual void clear ()

Clear the existing content.

\subsubsection{Detailed Description}

The bit-sliced index (O’Neil). It used the binary encoding.

\subsubsection{Member Function Documentation}

3.96.2.1 void ibis::slice::estimate (const ibis::qContinuousRange \& expr, ibis::bitvector \& lower, ibis::bitvector \& upper) const [virtual]

Computes an approximation of hits as a pair of lower and upper bounds.

\section{Parameters:}

expr the query expression to be evaluated.

lower a bitvector marking a subset of the hits. All rows marked with one (1) are definitely hits.

upper a bitvector marking a superset of the hits. All hits are marked with one, but some of the rows marked one may not be hits. If the variable upper is empty, the variable lower is assumed to contain the exact answer.

Reimplemented from ibis::relic.

3.96.2.2 long ibis::slice::evaluate (const ibis::qContinuousRange \& expr, ibis::bitvector \& hits) const [virtual]

To evaluate the exact hits.

On success, return the number of hits, otherwise a negative value is returned.

Reimplemented from ibis::relic.

\subsubsection{3 double ibis::slice::getSum () const [virtual]}

Compute the approximate sum of all the values indexed.

If it decides that computing the sum directly from the vertical partition is more efficient, it will return NaN immediately. Reimplemented from ibis::relic.

\subsubsection{4 void ibis::slice::print (std::ostream \& out) const [ virtua l]}

Prints human readable information.

Outputs information about the index as text to the specified output stream.

Reimplemented from ibis::relic. 


\subsubsection{5 void ibis::slice::read (ibis::fileManager::storage $*$ st) [virtual]}

Reconstruct an index from a piece of consecutive memory.

Unlike the implementations for other type indices, this function always reads all bit vectors.

Reimplemented from ibis::relic.

\subsubsection{6 void ibis::slice::read (const char $* \boldsymbol{f}) \quad$ [ virtual]}

Read the index contained in the file $\mathrm{f}$.

This function always reads all bitvectors.

Reimplemented from ibis::relic.

\subsubsection{7 void ibis::slice::write (const char $* \boldsymbol{d} \boldsymbol{t})$ const [ virtual]}

Save index to a file.

Outputs the index in a compact binary format to the named file or directory. The index file contains a header that can be identified by the function isIndex.

Reimplemented from ibis::relic.

The documentation for this class was generated from the following files:

- irelic.h

- islice.cpp

\subsection{7 ibis::tabele Class Reference}

A trivial class for table with one row and one column.

\#include <tab.h>

Inheritance diagram for ibis::tabele::

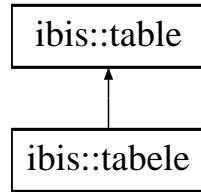

\section{Public Member Functions}

- virtual int buildIndex (const char $*$, const char $*$ )

Create the index for named column.

- virtual int buildIndexes (const char $*$ )

Create indexes for every column of the table.

- const char $*$ colName () const

- virtual stringList columnNames () const

Return column names.

- virtual typeList columnTypes () const

Return data types. 
- virtual ibis::table::cursor $*$ createCursor () const

Create a cursor object to perform row-wise data access.

- virtual void describe (std::ostream \&) const

Print a description of the table to the specified output stream.

- virtual int dump (std::ostream \&, const char $*$ ) const

Dump the values in ASCII form to the specified output stream.

- virtual void estimate (const char $*$ cond, uint64_t \&nmin, uint64_t \&nmax) const

Estimate the number of rows satisfying the selection conditions.

- virtual int64_t getColumnAsBytes (const char $*$, char $*$ ) const

Retrieve all values of the named column.

- virtual int64_t getColumnAsDoubles (const char $*$, double $*$ ) const

Retrieve all values of the named column.

- virtual int64_t getColumnAsFloats (const char $*$, float $*$ ) const

Retrieve all values of the named column.

- virtual int64_t getColumnAsInts (const char $*$, int32_t $*$ ) const

Retrieve all values of the named column.

- virtual int64_t getColumnAsLongs (const char $* \mathrm{cn}$, int64_t $*$ vals) const

Retrieve all values of the named column.

- virtual int64_t getColumnAsShorts (const char $*$, int16_t $*$ ) const

Retrieve all values of the named column.

- virtual int64_t getColumnAsStrings (const char $*$, std::vector $<$ std::string $>\&$ ) const

Retrieve the null-terminated strings as a vector of std::string objects.

- virtual int64_t getColumnAsUBytes (const char $*$, unsigned char $*$ ) const

Retrieve all values of the named column.

- virtual int64_t getColumnAsUInts (const char $* \mathrm{cn}$, uint32_t $*$ vals) const

Retrieve all values of the named column.

- virtual int64_t getColumnAsULongs (const char $* \mathrm{cn}$, uint64_t $*$ vals) const

Retrieve all values of the named column.

- virtual int64_t getColumnAsUShorts (const char $*$, uint16_t $*$ ) const

Retrieve all values of the named column.

- virtual long getHistogram (const char $*$, const char $*$, double, double, double, std::vector $<$ size_t $>\&$ ) const Compute the histogram of the named column.

- virtual long getHistogram2D (const char $*$, const char $*$, double, double, double, const char $*$, double, double, double, std::vector $<$ size_t $>\&$ ) const

Compute a two-dimension histogram on columns cname 1 and name2. 
- virtual long getHistogram3D (const char *, const char *, double, double, double, const char $*$, double, double, double, const char $*$, double, double, double, std::vector $<$ size_t $>\&$ ) const

Compute a three-dimensional histogram on the named columns.

- virtual table $*$ groupby (const stringList $\&$ ) const

Perform aggregate functions on the current table.

- virtual void indexSpec (const char $*$, const char $*$ )

Replace the current indexing option.

- virtual const char $*$ indexSpec (const char $*$ ) const

Retrieve the current indexing option.

- virtual size_t nColumns () const

- virtual uint64_t nRows () const

- virtual void orderby (const stringList \&)

Reorder the rows.

- virtual void reverseRows ()

Reverse the order of the rows.

- virtual table $*$ select (const char $*$, const char $*$ ) const

Given a set of column names and a set of selection conditions, compute another table that represents the selected values.

- tabele (uint64_t nr=0, const char $* \mathrm{~nm}=0$ )

- tabele (const char $*$ na, const char $*$ de, uint64_t nr, const char $* \mathrm{~nm}=0$ )

\section{Friends}

- class cursor

\section{Classes}

- class cursor

\subsubsection{Detailed Description}

A trivial class for table with one row and one column.

This type of table is generated when the select clause is "count $(*)$ ". This class could be replaced with ibis::tabula, however, treating the output of "count $(*)$ " as a one-row-and-one-column table is closer to the ODBC/JDBC convention.

\subsubsection{Member Function Documentation}

\subsubsection{1 virtual int ibis::tabele::buildIndex (const char $*$, const char $*$ ) [inline, virtual]}

Create the index for named column.

The existing index will be replaced. If an indexing option is not specified, it will use the internally recorded option for the named column or the table containing the column.

\section{Note:}

Unless any there is a specific instruction to not index a column, the querying functions will automatically build indices as necessary. However, as building an index is relatively expensive process, building an index on a column 
is on average about four or five times as expensive as reading the column from disk, this function is provided so that it is possible to build indexes beforehand.

Implements ibis::table.

\subsubsection{2 virtual int ibis::tabele::buildIndexes (const char $*$ ) [inline, virtual]}

Create indexes for every column of the table.

Existing indexes will be replaced. If an indexing option is not specified, the internally recorded options will be used. buildIndex

Implements ibis::table.

\subsubsection{3 int ibis::tabele::dump (std::ostream \&, const char $*$ ) const [inline, virtual]}

Dump the values in ASCII form to the specified output stream.

The default delimiter is coma (","), which produces Comma-Separated-Values (CSV).

Implements ibis::table.

3.97.2.4 void ibis::tabele::estimate (const char $*$ cond, uint64_t \& nmin, uint64_t \& nmax) const [in ine $_{\text {, }}$ virtual]

Estimate the number of rows satisfying the selection conditions.

The number of rows is between [nmin, nmax].

Implements ibis::table.

3.97.2.5 virtual int64_t ibis::tabele::getColumnAsBytes (const char $*$, char $*$ ) const [inline, virtual]

Retrieve all values of the named column.

The member functions of this class only support access to whole column at a time. Use table: : cursor class for row-wise accesses. For fixed-width data types, the raw pointers are used to point to the values to be returned. In these cases, the caller is responsible for allocating enough storage for the values to be returned.

Implements ibis::table.

3.97.2.6 virtual int64_t ibis::tabele::getColumnAsDoubles (const char $*$, double $*$ ) const [inline, virtual]

Retrieve all values of the named column.

The member functions of this class only support access to whole column at a time. Use table: : cursor class for row-wise accesses. For fixed-width data types, the raw pointers are used to point to the values to be returned. In these cases, the caller is responsible for allocating enough storage for the values to be returned.

Implements ibis::table.

3.97.2.7 virtual int64_t ibis::tabele::getColumnAsFloats (const char $*$, float $*$ ) const [in line, virtual ]

Retrieve all values of the named column.

The member functions of this class only support access to whole column at a time. Use table: : cursor class for row-wise accesses. For fixed-width data types, the raw pointers are used to point to the values to be returned. In these cases, the caller is responsible for allocating enough storage for the values to be returned.

Implements ibis::table. 
3.97.2.8 virtual int64_t ibis::tabele::getColumnAsInts (const char $*$, int32_t $*$ ) const [inline, virtua l ]

Retrieve all values of the named column.

The member functions of this class only support access to whole column at a time. Use table: : cursor class for row-wise accesses. For fixed-width data types, the raw pointers are used to point to the values to be returned. In these cases, the caller is responsible for allocating enough storage for the values to be returned.

Implements ibis::table.

3.97.2.9 virtual int64_t ibis::tabele::getColumnAsLongs (const char $* \boldsymbol{c n}$, int64_t $* v a l s)$ const [ in 1 ine, virtual]

Retrieve all values of the named column.

The member functions of this class only support access to whole column at a time. Use table: : cursor class for row-wise accesses. For fixed-width data types, the raw pointers are used to point to the values to be returned. In these cases, the caller is responsible for allocating enough storage for the values to be returned.

Implements ibis::table.

3.97.2.10 virtual int64_t ibis::tabele::getColumnAsShorts (const char $*$, int16_t *) const [in]ine, virtual]

Retrieve all values of the named column.

The member functions of this class only support access to whole column at a time. Use table: : cursor class for row-wise accesses. For fixed-width data types, the raw pointers are used to point to the values to be returned. In these cases, the caller is responsible for allocating enough storage for the values to be returned.

Implements ibis::table.

3.97.2.11 virtual int64_t ibis::tabele::getColumnAsStrings (const char $*$, std::vector $<$ std::string $>$ \&) const [inline, virtual]

Retrieve the null-terminated strings as a vector of std::string objects.

Both ibis::CATEGORY and ibis::TEXT types can be retrieved using this function.

Implements ibis::table.

3.97.2.12 virtual int64_t ibis::tabele::getColumnAsUBytes (const char $*$, unsigned char $*$ ) const $\quad[i n] i n e$, virtual]

Retrieve all values of the named column.

The member functions of this class only support access to whole column at a time. Use table: : cursor class for row-wise accesses. For fixed-width data types, the raw pointers are used to point to the values to be returned. In these cases, the caller is responsible for allocating enough storage for the values to be returned.

Implements ibis::table.

3.97.2.13 virtual int64_t ibis::tabele::getColumnAsUInts (const char $* \boldsymbol{c n}$, uint32_t $*$ vals) const [ i n ] i ne, virtual]

Retrieve all values of the named column.

The member functions of this class only support access to whole column at a time. Use table: : cursor class for row-wise accesses. For fixed-width data types, the raw pointers are used to point to the values to be returned. In these cases, the caller is responsible for allocating enough storage for the values to be returned.

Implements ibis::table. 
3.97.2.14 virtual int64_t ibis::tabele::getColumnAsULongs (const char $*$ cn, uint64_t $*$ vals) const [inline, virtual]

Retrieve all values of the named column.

The member functions of this class only support access to whole column at a time. Use table: : cursor class for row-wise accesses. For fixed-width data types, the raw pointers are used to point to the values to be returned. In these cases, the caller is responsible for allocating enough storage for the values to be returned.

Implements ibis::table.

3.97.2.15 virtual int64_t ibis::tabele::getColumnAsUShorts (const char $*$, uint16_t $*$ ) const [in]ine, virtual ]

Retrieve all values of the named column.

The member functions of this class only support access to whole column at a time. Use table: : cursor class for row-wise accesses. For fixed-width data types, the raw pointers are used to point to the values to be returned. In these cases, the caller is responsible for allocating enough storage for the values to be returned.

Implements ibis::table.

3.97.2.16 virtual long ibis::tabele::getHistogram (const char $*$, const char $*$, double, double, double, std::vector $<$ size_t $>\boldsymbol{\&})$ const [inline, virtual]

Compute the histogram of the named column.

This version uses the user specified bins: [begin, begin+stride) [begin+stride, begin $+2 *$ stride), .... A record is placed in bin

( $\mathrm{x}$ - begin) / stride, where the first bin is bin 0 . This gives a total of

(end - begin) / stride bins.

Note:

Records (rows) outside of the range [begin, end] are not counted.

Non-positive stride is considered as an error.

If end is less than begin, an empty array counts is returned along with return value 0 .

Implements ibis::table.

3.97.2.17 virtual long ibis::tabele::getHistogram2D (const char $*$, const char $*$, double, double, double, const char $*$, double, double, double, std::vector $<$ size_t $>\boldsymbol{\&})$ const $\quad$ [inline, virtual]

Compute a two-dimension histogram on columns cname1 and name2.

The bins along each dimension are defined the same way as in function getHistogram. The array counts stores the two-dimensional bins with the first dimension as the slow varying dimension following $\mathrm{C}$ convention for ordering multi-dimensional arrays.

Implements ibis::table.

3.97.2.18 virtual long ibis::tabele::getHistogram3D (const char $*$, const char $*$, double, double, double, const char $*$, double, double, double, const char $*$, double, double, double, std::vector $<$ size_t $>$ \&) const [inline, virtual]

Compute a three-dimensional histogram on the named columns.

The triplets < begin, end, stride $>$ are used the same ways in getHistogram and getHistogram2D. The three dimensional bins are linearized in count s with the first being the slowest varying dimension and the third being the fastest varying dimension following the $\mathrm{C}$ convention for ordering multi-dimensional arrays.

Implements ibis::table. 
3.97.2.19 virtual table $*$ ibis::tabele::groupby (const stringList \&) const [inline, virtual]

Perform aggregate functions on the current table.

It produces a new table. The list of strings passed to this function are interpreted as a set of names followed by a set of functions. Currently, only functions COUNT, AVG, MIN, MAX, and SUM are supported.

Implements ibis::table.

\subsubsection{0 virtual void ibis::tabele::indexSpec (const char $*$, const char $*$ ) [inline, virtual]}

Replace the current indexing option.

If no column name is specified, it resets the indexing option for the table.

Implements ibis::table.

\subsubsection{1 virtual const char* ibis::tabele::indexSpec (const char $*$ ) const [inline, virtual]}

Retrieve the current indexing option.

If no column name is specified, it retrieve the indexing option for the table.

Implements ibis::table.

\subsubsection{2 virtual void ibis::tabele::orderby (const stringList \&) [inline, virtual]}

Reorder the rows.

Sort the rows in ascending order of the columns specified in the list of column names. This function is not designated const because though it does not change the content in SQL logic, but it may change internal representations.

\section{Note:}

If an empty list is passed to this function, it will reorder rows using all columns with the column having the smallest number of distinct values first.

Implements ibis::table.

The documentation for this class was generated from the following file:

- tab.h

\subsection{8 ibis::table Class Reference}

The abstract table class.

\#include <table.h>

Inheritance diagram for ibis::table::

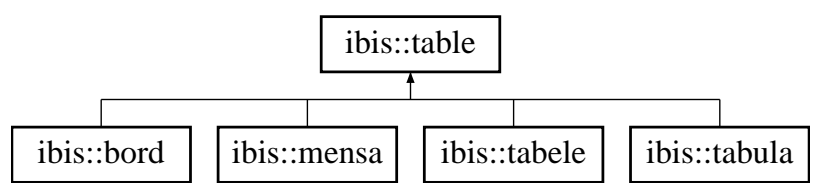

\section{Public Types}

- typedef std::map $<$ const char $*$, ibis::TYPE_T, ibis::lessi $>$ namesTypes 
An associate array of names and types.

- typedef std::vector $<$ const char $*>$ stringList A list of strings.

- typedef std::vector $<$ ibis::TYPE_T $>$ typeList

A list of data types.

\section{Public Member Functions}

- virtual stringList columnNames () const=0

Return column names.

- virtual typeList columnTypes () const=0

Return data types.

- virtual cursor $*$ createCursor () const $=0$

Create a cursor object to perform row-wise data access.

- virtual void describe (std::ostream \&) const=0

Print a description of the table to the specified output stream.

- virtual const char $*$ description () const

Free text description.

- virtual int dump (std::ostream \&out, const char $*$ del=", ") const=0

Dump the values in ASCII form to the specified output stream.

- virtual void estimate (const char $*$ cond, uint64_t \&nmin, uint64_t \&nmax) const $=0$

Estimate the number of rows satisfying the selection conditions.

- virtual table $*$ groupby (const char $*$ ) const

Perform group-by operation.

- virtual table $*$ groupby (const stringList $\&$ ) const $=0$

Perform aggregate functions on the current table.

- virtual const char $*$ name () const

Name of the table object.

- virtual size_t nColumns () const $=0$

- virtual uint64_t nRows () const=0

- virtual void orderby (const char $*$ )

Reorder the rows. The column names are separated by comma.

- virtual void orderby (const stringList \&) $=0$

Reorder the rows.

- virtual void reverseRows ()$=0$

Reverse the order of the rows.

- virtual table $*$ select (const char $*$ sel, const char $*$ cond) const $=0$ 
Given a set of column names and a set of selection conditions, compute another table that represents the selected values.

- virtual $\sim$ table ()

Destructor.

- virtual int buildIndex (const char $*$ colname, const char $*$ option $=0$ ) $=0$

Create the index for named column.

- virtual int buildIndexes (const char $*$ options $=0$ ) $=0$

Create indexes for every column of the table.

- virtual void indexSpec (const char $*$ opt, const char $*$ colname $=0)=0$

Replace the current indexing option.

- virtual const char $*$ indexSpec (const char $*$ colname $=0$ ) const $=0$

Retrieve the current indexing option.

- virtual int64_t getColumnAsBytes (const char $*$ cname, char $*$ vals) const $=0$

Retrieve all values of the named column.

- virtual int64_t getColumnAsDoubles (const char $*$ cname, double $*$ vals) const $=0$

Retrieve all values of the named column.

- virtual int64_t getColumnAsFloats (const char $*$ cname, float $*$ vals) const $=0$

Retrieve all values of the named column.

- virtual int64_t getColumnAsInts (const char $*$ cname, int32_t $*$ vals) const $=0$

Retrieve all values of the named column.

- virtual int64_t getColumnAsLongs (const char $*$ cname, int64_t $*$ vals) const=0

Retrieve all values of the named column.

- virtual int64_t getColumnAsShorts (const char $*$ cname, int16_t $*$ vals) const $=0$

Retrieve all values of the named column.

- virtual int64_t getColumnAsStrings (const char $*$ cname, std::vector $<$ std::string $>$ \&vals) const=0

Retrieve the null-terminated strings as a vector of std::string objects.

- virtual int64_t getColumnAsUBytes (const char $*$ cname, unsigned char $*$ vals) const $=0$

Retrieve all values of the named column.

- virtual int64_t getColumnAsUInts (const char $*$ cname, uint32_t $*$ vals) const $=0$

Retrieve all values of the named column.

- virtual int64_t getColumnAsULongs (const char $*$ cname, uint64_t $*$ vals) const=0

Retrieve all values of the named column.

- virtual int64_t getColumnAsUShorts (const char $*$ cname, uint16_t $*$ vals) const=0

Retrieve all values of the named column.

- virtual long getHistogram (const char $*$ constraints, const char $*$ cname, double begin, double end, double stride, std::vector $<$ size_t $>$ \& counts) const $=0$

Compute the histogram of the named column. 
- virtual long getHistogram2D (const char $*$ constraints, const char $*$ cname1, double begin 1 , double end1, double stride1, const char $*$ cname2, double begin2, double end2, double stride2, std::vector $<$ size_t $>\&$ counts) const $=0$

Compute a two-dimension histogram on columns cname1 and name2.

- virtual long getHistogram3D (const char $*$ constraints, const char $*$ cname1, double begin1, double end1, double stride1, const char $*$ cname 2 , double begin2, double end2, double stride2, const char $*$ cname 3 , double begin3, double end3, double stride3, std::vector $<$ size_t $>\&$ counts) const $=0$

Compute a three-dimensional histogram on the named columns.

\section{Static Public Member Functions}

- static ibis::table $*$ create (const char $* \operatorname{dir} 1$, const char $* \operatorname{dir} 2$ )

Create a table object from a pair of data directories.

- static ibis::table $*$ create (const char $*$ dir)

Create a table object from the specified data directory.

\section{Protected Member Functions}

- void parseNames (char $*$ in, stringList \&out) const

Parse a string into a set of names.

- table (const char $*$ na, const char $*$ de)

Copy constructor.

- table ()

The default constructor.

\section{Protected Attributes}

- std::string desc_

Description of the table.

- std::string name_

Name of the table.

\section{Classes}

- class cursor

Cursor class for row-wise data accesses.

- struct row

A simple struct for storing a row of a table. 


\subsubsection{Detailed Description}

The abstract table class.

This is an abstract base class that defines the common operations on a data table. Conceptually, data records in a table is organized into rows and columns. A query on a table produces a filtered version of the table. In many database systems this is known as a view on a table. All data tables and views are logically treated as specialization of this ibis::table class.

\subsubsection{Member Function Documentation}

\subsubsection{1 virtual int ibis::table::buildIndex (const char $*$ colname, const char $*$ option $=0) \quad$ [pure virtual]}

Create the index for named column.

The existing index will be replaced. If an indexing option is not specified, it will use the internally recorded option for the named column or the table containing the column.

\section{Note:}

Unless any there is a specific instruction to not index a column, the querying functions will automatically build indices as necessary. However, as building an index is relatively expensive process, building an index on a column is on average about four or five times as expensive as reading the column from disk, this function is provided so that it is possible to build indexes beforehand.

Implemented in ibis::bord, ibis::mensa, ibis::tabula, and ibis::tabele.

\subsubsection{2 virtual int ibis::table::buildIndexes (const char $*$ options $=0$ ) [pure virtual]}

Create indexes for every column of the table.

Existing indexes will be replaced. If an indexing option is not specified, the internally recorded options will be used. buildIndex

Implemented in ibis::bord, ibis::mensa, ibis::tabula, and ibis::tabele.

\subsubsection{3 ibis::table $*$ ibis::table::create (const char $* \operatorname{dir} 1$, const char $* \operatorname{dir} 2) \quad$ [ static ]}

Create a table object from a pair of data directories.

This table maintains two sets of data files so that it can continue to process queries using existing data records while accepting new data records.

\subsubsection{4 virtual int ibis::table::dump (std::ostream \& out, const char $*$ del $=$ ", ") const [pure virtual]}

Dump the values in ASCII form to the specified output stream.

The default delimiter is coma (","), which produces Comma-Separated-Values (CSV).

Implemented in ibis::bord, ibis::mensa, ibis::tabula, and ibis::tabele.

3.98.2.5 virtual void ibis::table::estimate (const char $*$ cond, uint64_t \& nmin, uint64_t \& nmax) const [pure virtual]

Estimate the number of rows satisfying the selection conditions.

The number of rows is between [nmin, nmax].

Implemented in ibis::bord, ibis::mensa, ibis::tabula, and ibis::tabele. 
3.98.2.6 virtual int64_t ibis::table::getColumnAsBytes (const char $*$ cname, char $*$ vals) const [pure virtual]

Retrieve all values of the named column.

The member functions of this class only support access to whole column at a time. Use table: : cursor class for row-wise accesses. For fixed-width data types, the raw pointers are used to point to the values to be returned. In these cases, the caller is responsible for allocating enough storage for the values to be returned.

Implemented in ibis::bord, ibis::mensa, ibis::tabula, and ibis::tabele.

\subsubsection{7 virtual int64_t ibis::table::getColumnAsDoubles (const char $*$ cname, double $*$ vals) const [pure virtual]}

Retrieve all values of the named column.

The member functions of this class only support access to whole column at a time. Use table: : cursor class for row-wise accesses. For fixed-width data types, the raw pointers are used to point to the values to be returned. In these cases, the caller is responsible for allocating enough storage for the values to be returned.

Implemented in ibis::bord, ibis::mensa, ibis::tabula, and ibis::tabele.

3.98.2.8 virtual int64_t ibis::table::getColumnAsFloats (const char $*$ cname, float $*$ vals) const [pure virtual]

Retrieve all values of the named column.

The member functions of this class only support access to whole column at a time. Use table: : cursor class for row-wise accesses. For fixed-width data types, the raw pointers are used to point to the values to be returned. In these cases, the caller is responsible for allocating enough storage for the values to be returned.

Implemented in ibis::bord, ibis::mensa, ibis::tabula, and ibis::tabele.

3.98.2.9 virtual int64_t ibis::table::getColumnAsInts (const char $*$ cname, int32_t $*$ vals) const [pure virtual]

Retrieve all values of the named column.

The member functions of this class only support access to whole column at a time. Use table: : cursor class for row-wise accesses. For fixed-width data types, the raw pointers are used to point to the values to be returned. In these cases, the caller is responsible for allocating enough storage for the values to be returned.

Implemented in ibis::bord, ibis::mensa, ibis::tabula, and ibis::tabele.

3.98.2.10 virtual int64_t ibis::table::getColumnAsLongs (const char $*$ cname, int64_t $*$ vals) const $\quad$ [pure virtual]

Retrieve all values of the named column.

The member functions of this class only support access to whole column at a time. Use table: : cursor class for row-wise accesses. For fixed-width data types, the raw pointers are used to point to the values to be returned. In these cases, the caller is responsible for allocating enough storage for the values to be returned.

Implemented in ibis::bord, ibis::mensa, ibis::tabula, and ibis::tabele.

3.98.2.11 virtual int64_t ibis::table::getColumnAsShorts (const char $*$ cname, int16_t $*$ vals) const [pure virtual]

Retrieve all values of the named column.

The member functions of this class only support access to whole column at a time. Use table: : cursor class for row-wise accesses. For fixed-width data types, the raw pointers are used to point to the values to be returned. In these cases, the caller is responsible for allocating enough storage for the values to be returned. 
Implemented in ibis::bord, ibis::mensa, ibis::tabula, and ibis::tabele.

3.98.2.12 virtual int64_t ibis::table::getColumnAsStrings (const char $*$ cname, std::vector $<$ std::string $>\&$ vals) const [pure virtual]

Retrieve the null-terminated strings as a vector of std::string objects.

Both ibis::CATEGORY and ibis::TEXT types can be retrieved using this function.

Implemented in ibis::bord, ibis::mensa, ibis::tabula, and ibis::tabele.

3.98.2.13 virtual int64_t ibis::table::getColumnAsUBytes (const char $*$ cname, unsigned char $*$ vals) const [pure virtual]

Retrieve all values of the named column.

The member functions of this class only support access to whole column at a time. Use table: : cursor class for row-wise accesses. For fixed-width data types, the raw pointers are used to point to the values to be returned. In these cases, the caller is responsible for allocating enough storage for the values to be returned.

Implemented in ibis::bord, ibis::mensa, ibis::tabula, and ibis::tabele.

3.98.2.14 virtual int64_t ibis::table::getColumnAsUInts (const char $*$ cname, uint32_t $*$ vals) const [pure virtual]

Retrieve all values of the named column.

The member functions of this class only support access to whole column at a time. Use table: : cursor class for row-wise accesses. For fixed-width data types, the raw pointers are used to point to the values to be returned. In these cases, the caller is responsible for allocating enough storage for the values to be returned.

Implemented in ibis::bord, ibis::mensa, ibis::tabula, and ibis::tabele.

3.98.2.15 virtual int64_t ibis::table::getColumnAsULongs (const char $*$ cname, uint64_t $*$ vals) const $\quad$ [pure virtual]

Retrieve all values of the named column.

The member functions of this class only support access to whole column at a time. Use table: : cursor class for row-wise accesses. For fixed-width data types, the raw pointers are used to point to the values to be returned. In these cases, the caller is responsible for allocating enough storage for the values to be returned.

Implemented in ibis::bord, ibis::mensa, ibis::tabula, and ibis::tabele.

3.98.2.16 virtual int64_t ibis::table::getColumnAsUShorts (const char $*$ cname, uint16_t $*$ vals) const [pure virtual]

Retrieve all values of the named column.

The member functions of this class only support access to whole column at a time. Use table: : cursor class for row-wise accesses. For fixed-width data types, the raw pointers are used to point to the values to be returned. In these cases, the caller is responsible for allocating enough storage for the values to be returned.

Implemented in ibis::bord, ibis::mensa, ibis::tabula, and ibis::tabele.

3.98.2.17 virtual long ibis::table::getHistogram (const char $*$ constraints, const char $*$ cname, double begin, double end, double stride, std::vector $<$ size_t $>$ \& counts) const $\quad$ [pure virtual]

Compute the histogram of the named column.

This version uses the user specified bins: [begin, begin+stride) [begin+stride, begin $+2 *$ stride), .... A record is placed in bin 
( $\mathrm{x}$ - begin) / stride, where the first bin is bin 0 . This gives a total of

(end - begin) / stride bins.

Note:

Records (rows) outside of the range [begin, end] are not counted.

Non-positive stride is considered as an error.

If end is less than begin, an empty array counts is returned along with return value 0.

Implemented in ibis::bord, ibis::mensa, ibis::tabula, and ibis::tabele.

3.98.2.18 virtual long ibis::table::getHistogram2D (const char $*$ constraints, const char $*$ cname1, double begin1, double end1, double stride1, const char $*$ cname 2 , double begin2, double end2, double stride2, std::vector $<$ size_t $>$ \& counts) const [pure virtual]

Compute a two-dimension histogram on columns cname1 and name2.

The bins along each dimension are defined the same way as in function getHistogram. The array counts stores the two-dimensional bins with the first dimension as the slow varying dimension following $\mathrm{C}$ convention for ordering multi-dimensional arrays.

Implemented in ibis::bord, ibis::mensa, ibis::tabula, and ibis::tabele.

3.98.2.19 virtual long ibis::table::getHistogram3D (const char $*$ constraints, const char $*$ cname1, double begin1, double end1, double stride1, const char $*$ cname2, double begin2, double end2, double stride2, const char $*$ cname3, double begin3, double end3, double stride3, std::vector $<$ size_t $>$ \& counts) const [pure virtual]

Compute a three-dimensional histogram on the named columns.

The triplets < begin, end, stride $>$ are used the same ways in getHistogram and getHistogram2D. The three dimensional bins are linearized in count s with the first being the slowest varying dimension and the third being the fastest varying dimension following the $\mathrm{C}$ convention for ordering multi-dimensional arrays.

Implemented in ibis::bord, ibis::mensa, ibis::tabula, and ibis::tabele.

3.98.2.20 ibis::table $*$ ibis::table::groupby (const char $*$ ) const [inline, virtual]

Perform group-by operation.

The column names and operations are separated by comma.

\subsubsection{1 virtual table $*$ ibis::table::groupby (const stringList \&) const [pure virtual]}

Perform aggregate functions on the current table.

It produces a new table. The list of strings passed to this function are interpreted as a set of names followed by a set of functions. Currently, only functions COUNT, AVG, MIN, MAX, and SUM are supported.

Implemented in ibis::bord, ibis::mensa, ibis::tabula, and ibis::tabele.

3.98.2.22 virtual void ibis::table::indexSpec (const char $*$ opt, const char $*$ colname $=0$ ) [pure virtual ]

Replace the current indexing option.

If no column name is specified, it resets the indexing option for the table.

Implemented in ibis::bord, ibis::mensa, ibis::tabula, and ibis::tabele. 


\subsubsection{3 virtual const char $*$ ibis::table::indexSpec (const char $*$ colname $=0)$ const $\quad$ pure virtual]}

Retrieve the current indexing option.

If no column name is specified, it retrieve the indexing option for the table.

Implemented in ibis::bord, ibis::mensa, ibis::tabula, and ibis::tabele.

\subsubsection{4 virtual void ibis::table::orderby (const stringList \&) [pure virtual]}

Reorder the rows.

Sort the rows in ascending order of the columns specified in the list of column names. This function is not designated const because though it does not change the content in SQL logic, but it may change internal representations.

\section{Note:}

If an empty list is passed to this function, it will reorder rows using all columns with the column having the smallest number of distinct values first.

Implemented in ibis::bord, ibis::mensa, ibis::tabula, and ibis::tabele.

\subsubsection{5 void ibis::table::parseNames (char $*$ in, stringList \& out) const [protected]}

Parse a string into a set of names.

Some bytes may be turned into 0 to mark the end of names or functions.

The documentation for this class was generated from the following files:

- table.h

- mensa.cpp

\subsection{9 ibis::table::cursor Class Reference}

Cursor class for row-wise data accesses.

\#include <table.h>

\section{Public Member Functions}

- virtual ibis::table::stringList columnNames () const=0

- virtual ibis::table::typeList columnTypes () const=0

- virtual int dump (std::ostream \&out, const char $*$ del=", ") const=0

Print out the values of the current row.

- virtual int fetch (uint64_t rownum, ibis::table::row $\&$ ) =0

Fetch the content of the specified row and make that row the current row as well.

- virtual int fetch (ibis::table::row $\&)=0$

Fetch the content of the next row and make the next row as the current row as well.

- virtual int fetch (uint64_t rownum) $=0$

Make the specified row in the data set available for retrieval.

- virtual int fetch ()$=0$

Make the next row of the data set available for retrieval. 
- virtual int getColumnAsByte (size_t cnum, char $*$ vals) const=0

This version of getColumnAsTTT directly use the column number, i.e., the position of a column in the list returned by function columnNames or columnTypes.

- virtual int getColumnAsByte (const char $*$ cname, char $*$ ) const=0

Retrieve the value of the named column.

- virtual int getColumnAsDouble (size_t cnum, double $*$ vals) const $=0$

- virtual int getColumnAsDouble (const char $*$ cname, double $*$ ) const $=0$

- virtual int getColumnAsFloat (size_t cnum, float $*$ vals) const $=0$

- virtual int getColumnAsFloat (const char $*$ cname, float $*$ ) const $=0$

- virtual int getColumnAsInt (size_t cnum, int32_t $*$ vals) const $=0$

- virtual int getColumnAsInt (const char $*$ cname, int32_t $*$ ) const $=0$

- virtual int getColumnAsLong (size_t cnum, int64_t $*$ vals) const $=0$

- virtual int getColumnAsLong (const char $*$ cname, int64_t $*$ ) const $=0$

- virtual int getColumnAsShort (size_t cnum, int16_t $*$ vals) const $=0$

- virtual int getColumnAsShort (const char $*$ cname, int16_t $*$ ) const $=0$

- virtual int getColumnAsString (size_t cnum, std::string \& vals) const=0

- virtual int getColumnAsString (const char $*$ cname, std::string \&) const=0

- virtual int getColumnAsUByte (size_t cnum, unsigned char $*$ vals) const $=0$

- virtual int getColumnAsUByte (const char $*$ cname, unsigned char $*$ ) const $=0$

- virtual int getColumnAsUInt (size_t cnum, uint32_t $*$ vals) const $=0$

- virtual int getColumnAsUInt (const char $*$ cname, uint32_t $*$ ) const $=0$

- virtual int getColumnAsULong (size_t cnum, uint64_t $*$ vals) const=0

- virtual int getColumnAsULong (const char $*$ cname, uint64_t $*$ ) const $=0$

- virtual int getColumnAsUShort (size_t cnum, uint $16 \_t * v a l s$ ) const $=0$

- virtual int getColumnAsUShort (const char $*$ cname, uint16_t $*$ ) const $=0$

- virtual uint64_t getCurrentRowNumber () const=0

Return the current row number.

- virtual size_t nColumns () const $=0$

- virtual uint64_t nRows () const=0

\section{Protected Member Functions}

- $\operatorname{cursor}$ (const cursor \&)

- cursor \& operator= $($ const cursor $\&)$

\subsubsection{Detailed Description}

Cursor class for row-wise data accesses.

Note:

Note that this cursor is associated with a table object and can only iterate overall rows of a table. To iterate an arbitrary selection of rows, use the selection to create a new table and then iterate over the new table.

\subsubsection{Member Function Documentation}

\subsubsection{1 virtual int ibis::table::cursor::fetch (uint64_t rownum) [pure virtual]}

Make the specified row in the data set available for retrieval.

Returns 0 if the specified row is found, returns a negative number to indicate error, such as rownum out of range (-1). 


\subsubsection{2 virtual int ibis::table::cursor::fetch () [pure virtual]}

Make the next row of the data set available for retrieval.

Returns 0 if successful, returns a negative number to indicate error.

3.99.2.3 virtual int ibis::table::cursor::getColumnAsByte (size_t cnum, char $*$ vals) const [pure virtual]

This version of getColumnAsTTT directly use the column number, i.e., the position of a column in the list returned by function columnNames or columnTypes.

This version of the data access function may be able to avoid the name lookup and reduce the execution time.

3.99.2.4 virtual int ibis::table::cursor::getColumnAsByte (const char $*$ cname, char $*$ ) const [pure virtual]

Retrieve the value of the named column.

Note:

Note the cost of name lookup is likely to dominate the total cost of such a function.

\subsubsection{5 virtual uint64_t ibis::table::cursor::getCurrentRowNumber () const [pure virtual]}

Return the current row number.

Rows in a data set are numbered [0

- nRows ( $)-1]$. If the cursor is not ready, such as before the first call to fetch or function fetch returned an error, this function return the same value as function nRows.

The documentation for this class was generated from the following file:

- table.h

\subsection{0 ibis::table::row Struct Reference}

A simple struct for storing a row of a table.

\#include <table.h>

\section{Public Member Functions}

- void clear ()

- void clearValues ()

\section{Public Attributes}

- std::vector $<$ std::string $>$ bytesnames For ibis::BYTE.

- std::vector $<$ signed char $>$ bytesvalues

- std::vector $<$ std::string $>$ catsnames

For ibis::CATEGORY. 
- std::vector $<$ std::string > catsvalues

- std::vector $<$ std::string $>$ doublesnames

For ibis::DOUBLE.

- std::vector $<$ double $>$ doublesvalues

- std::vector $<$ std::string $>$ floatsnames

For ibis::FLOAT.

- std::vector $<$ float $>$ floatsvalues

- std::vector $<$ std::string $>$ intsnames

For ibis::INT.

- std::vector $<$ int32_t $>$ intsvalues

- std::vector $<$ std::string $>$ longsnames

For ibis::LONG.

- std::vector $<$ int64_t $>$ longsvalues

- std::vector $<$ std::string $>$ shortsnames For ibis::SHORT.

- std::vector $<$ int16_t $>$ shortsvalues

- std::vector $<$ std::string $>$ textsnames For ibis::TEXT.

- std::vector $<$ std::string $>$ textsvalues

- std::vector $<$ std::string $>$ ubytesnames For ibis::UBYTE.

- std::vector $<$ unsigned char $>$ ubytesvalues

- std::vector $<$ std::string $>$ uintsnames For ibis::UINT.

- std::vector $<$ uint32_t $>$ uintsvalues

- std::vector $<$ std::string $>$ ulongsnames For ibis::ULONG.

- std::vector $<$ uint64_t $>$ ulongsvalues

- std::vector $<$ std::string $>$ ushortsnames For ibis::USHORT.

- std::vector $<$ uint16_t $>$ ushortsvalues

\subsubsection{Detailed Description}

A simple struct for storing a row of a table.

The documentation for this struct was generated from the following file:

- table.h

\subsection{1 ibis::tableList Class Reference}

A list of tables.

\#include <table.h> 


\section{Public Types}

- typedef tableSet::const_iterator iterator

- typedef std::map < const char $*$, ibis::table $*$, ibis::lessi $>$ tableSet

\section{Public Member Functions}

- void add (ibis::table $* \&$ tb)

Add a new table object to the list.

- iterator begin () const

Return the iterator to the first table.

- bool empty () const

Is the list empty? Returns true of the list is empty, otherwise returns false.

- iterator end () const

Return the iterator to the end of the list.

- const ibis::table $*$ operator[ ] (const char $*$ tname) const

Find the named table.

- void remove (const char $*$ tname)

Remove the named data table from the list.

- size_t size () const

Return the number of tables in the list.

- tableList ()

Default constructor.

- tableList ()

Destructor. Delete all table objects.

\subsubsection{Detailed Description}

A list of tables.

It supports simple lookup through operator[] and manages the table objects passed to it. Most functions are simply wrappers on std::map.

\subsubsection{Member Function Documentation}

\subsubsection{1 void ibis::tableList::add (ibis::table $* \& \boldsymbol{t b}$ ) [ in line ]}

Add a new table object to the list.

Transfers the control of the object to the tableList. If the name of the table already exists, the existing table will be passed back out, otherwise, the argument tb is set to null. In either case, the caller can call delete on the variable and should do so to avoid memory leak. 


\subsubsection{2 iterator ibis::tableList::end () const [inline]}

Return the iterator to the end of the list.

Following STL convention, the end is always one past the last element in the list.

3.101.2.3 ] const ibis::table $*$ ibis::tableList::operator[] (const char $*$ tname) const [in line ]

Find the named table.

Returns null pointer if no table with the given name is found.

\subsubsection{4 void ibis::tableList::remove (const char $*$ tname) $\quad$ [inline ]}

Remove the named data table from the list.

The destructor of this function automatically clean up all table objects, there is no need to explicit remove them.

The documentation for this class was generated from the following file:

- table.h

\subsection{2 ibis::tablex Class Reference}

The class for expandable tables.

\#include <table.h>

Inheritance diagram for ibis::tablex::

ibis::tablex

ibis::tafel

\section{Public Member Functions}

- virtual int addColumn (const char $*$ cname, ibis::TYPE_T ctype, const char $*$ cdesc) $=0$

- virtual int addColumn (const char $*$ cname, ibis::TYPE_T ctype) $=0$ Add a column.

- virtual int append (const char $*$ cname, uint64_t begin, uint64_t end, void $*$ values) $=0$ Add values to the named column.

- virtual int appendRow (const char $*$ line, const char $*$ delimiters $=0)=0$ Append a row stored in ASCII form.

- virtual int appendRow (const ibis::table::row $\&$ ) $=0$ Add one row.

- virtual int appendRows (const std::vector $<$ ibis::table::row $>\&$ ) $=0$ Add multiple rows.

- virtual int readCSV (const char $*$ filename, const char $*$ delimiters $=0$ ) $=0$ Read the content of the specified as comma-separated values. 
- virtual int write (const char $*$ dir, const char $*$ thame, const char $*$ tdesc) const $=0$

Write the in-memory data records to the specified directory on disk.

\section{Static Public Member Functions}

- static ibis::tablex $*$ create ()

Create a minimalistic table exclusively for entering new records.

- static ibis::tablex $*$ makeExtensible (ibis::table $* \mathrm{t}$ )

Make the incoming table expandable. Not yet implemented.

\subsubsection{Detailed Description}

The class for expandable tables.

Note:

Each function that returns an integer returns 0 in case of success, a negative value in case error and a positive number as advisory information.

\subsubsection{Member Function Documentation}

3.102.2.1 virtual int ibis::tablex::append (const char $*$ cname, uint64_t begin, uint64_t end, void $*$ values) [pure virtual]

Add values to the named column.

The column name must be in the table already. The first value is to be placed at row begin (the row numbers start with 0 ) and the last value before row end. The array values must contain values of the correct type corresponding to the type specified before.

Note:

Since each column may have different number of rows filled, the number of rows in the table is considered to be the maximum number of rows filled of all columns.

This function can not be used to introduce new columns in a table. A new column must be added with addcolumn.

appendRow

Implemented in ibis::tafel.

3.102.2.2 virtual int ibis::tablex::appendRow (const char $*$ line, const char $*$ delimiters $=0$ ) [pure virtual]

Append a row stored in ASCII form.

The ASCII form of the values are assumed to be separated by comma (,) or space, but additional delimiters may be added through the second argument.

Implemented in ibis::tafel. 


\subsubsection{3 virtual int ibis::tablex::appendRow (const ibis::table::row \&) [pure virtual]}

Add one row.

If an array of names has the same number of elements as the array of values, the names are used as column names. If the names are not specified explicitly, the values are assigned to the columns of the same data type in the order as they are specified through addColumn or if the same order as they are recreated from an existing dataset (which is typically alphabetical).

\section{Note:}

The column names are not case-sensitive.

Like append, this function can not be used to introduce new columns in a table. A new column must be added with addColumn.

Since the various columns may have different numbers of rows filled, the number of rows in the table is assumed to the largest number of rows filled so far. The new row appended here increases the number of rows in the table by 1 . The unfilled rows are assumed to be null.

A null value of an integer column is recorded as the maximum possible of the type of integer. A null value of a floating-point valued column is recorded as a quiet $\mathrm{NaN}$ (Not-a-Number). A null value of a string-valued column is recorded as an empty string. In all cases, a null mask is used to indicate that they are null values.

Implemented in ibis::tafel.

\subsubsection{4 virtual int ibis::tablex::appendRows (const std::vector $<$ ibis::table::row $>$ \&) [pure virtual]}

Add multiple rows.

Rows in the incoming vector are processed on after another. The ordering of the values in earlier rows are automatically carried over to the later rows until another set of names is specified. appendRow

Implemented in ibis::tafel.

3.102.2.5 virtual int ibis::tablex::readCSV (const char $*$ filename, const char $*$ delimiters $=0$ ) $\quad$ [pure virtual]

Read the content of the specified as comma-separated values.

Append the records to this table. By default the records are delimited by comma (,) and blank space. One may specify additional delimiters using the second argument.

Implemented in ibis::tafel.

3.102.2.6 virtual int ibis::tablex::write (const char $*$ dir, const char $*$ tname, const char $*$ tdesc) const [pure virtual]

Write the in-memory data records to the specified directory on disk.

If the table name (tname) is a null string or an empty, the last component of the directory name is used. If the description (tdesc) is a null string or an empty string, a time stamp will be printed in its place. If the specified directory already contains data, the new records will be appended to the existing data. In this case, the table name specified here will overwrite the existing name, but the existing name and description will be retained if the current arguments are null strings or empty strings. The data type associated with this table will overwrite the existing data type information.

Implemented in ibis::tafel.

The documentation for this class was generated from the following files:

- table.h

- tafel.cpp 


\subsection{3 ibis::tabula Class Reference}

A trivial class for table with no columns.

\#include <tab.h>

Inheritance diagram for ibis::tabula::

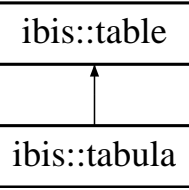

\section{Public Member Functions}

- virtual int buildIndex (const char $*$, const char $*$ )

Create the index for named column.

- virtual int buildIndexes (const char $*$ )

Create indexes for every column of the table.

- virtual stringList columnNames () const

Return column names.

- virtual typeList columnTypes () const

Return data types.

- virtual ibis::table::cursor $*$ createCursor () const

Create a cursor object to perform row-wise data access.

- virtual void describe (std::ostream \&) const

Print a description of the table to the specified output stream.

- virtual int dump (std::ostream \&, const char $*$ ) const

Dump the values in ASCII form to the specified output stream.

- virtual void estimate (const char *cond, uint64_t \&nmin, uint64_t \&nmax) const

Estimate the number of rows satisfying the selection conditions.

- virtual int64_t getColumnAsBytes (const char $*$, char $*$ ) const

Retrieve all values of the named column.

- virtual int64_t getColumnAsDoubles (const char $*$, double $*$ ) const

Retrieve all values of the named column.

- virtual int64_t getColumnAsFloats (const char $*$, float $*$ ) const

Retrieve all values of the named column.

- virtual int64_t getColumnAsInts (const char $*$, int32_t $*$ ) const

Retrieve all values of the named column.

- virtual int64_t getColumnAsLongs (const char $*$, int64_t $*$ ) const

Retrieve all values of the named column. 
- virtual int64_t getColumnAsShorts (const char $*$, int16_t $*$ ) const Retrieve all values of the named column.

- virtual int64_t getColumnAsStrings (const char $*$, std::vector $<$ std::string $>\&$ ) const Retrieve the null-terminated strings as a vector of std::string objects.

- virtual int64_t getColumnAsUBytes (const char $*$, unsigned char $*$ ) const Retrieve all values of the named column.

- virtual int64_t getColumnAsUInts (const char $*$, uint32_t *) const Retrieve all values of the named column.

- virtual int64_t getColumnAsULongs (const char $*$, uint64_t $*$ ) const Retrieve all values of the named column.

- virtual int64_t getColumnAsUShorts (const char $*$, uint16_t $*$ ) const Retrieve all values of the named column.

- virtual long getHistogram (const char $*$, const char $*$, double, double, double, std::vector $<$ size_t $>\&$ ) const Compute the histogram of the named column.

- virtual long getHistogram2D (const char $*$, const char $*$, double, double, double, const char $*$, double, double, double, std::vector $<$ size_t $>$ \&) const

Compute a two-dimension histogram on columns cname1 and name2.

- virtual long getHistogram3D (const char $*$, const char $*$, double, double, double, const char $*$, double, double, double, const char $*$, double, double, double, std::vector $<$ size_t $>\&$ ) const

Compute a three-dimensional histogram on the named columns.

- virtual table $*$ groupby (const stringList $\&$ ) const

Perform aggregate functions on the current table.

- virtual void indexSpec (const char $*$, const char $*$ )

Replace the current indexing option.

- virtual const char $*$ indexSpec (const char $*$ ) const

Retrieve the current indexing option.

- virtual size_t nColumns () const

- virtual uint64_t nRows () const

- virtual void orderby (const stringList \&)

Reorder the rows.

- virtual void reverseRows ()

Reverse the order of the rows.

- virtual table $*$ select (const char $*$, const char $*$ ) const

Given a set of column names and a set of selection conditions, compute another table that represents the selected values.

- tabula (size_t $\mathrm{nr}=0)$

- tabula (const char $*$ na, const char $*$ de, uint64_t nr) 


\section{Classes}

- class cursor

\subsubsection{Detailed Description}

A trivial class for table with no columns.

This type of table is generated when the select clause is blank or not specified.

\subsubsection{Member Function Documentation}

\subsubsection{1 virtual int ibis::tabula::buildIndex (const char $*$, const char $*$ ) [inline, virtual]}

Create the index for named column.

The existing index will be replaced. If an indexing option is not specified, it will use the internally recorded option for the named column or the table containing the column.

Note:

Unless any there is a specific instruction to not index a column, the querying functions will automatically build indices as necessary. However, as building an index is relatively expensive process, building an index on a column is on average about four or five times as expensive as reading the column from disk, this function is provided so that it is possible to build indexes beforehand.

Implements ibis::table.

\subsubsection{2 virtual int ibis::tabula::buildIndexes (const char $*$ ) [inline, virtual]}

Create indexes for every column of the table.

Existing indexes will be replaced. If an indexing option is not specified, the internally recorded options will be used. buildIndex

Implements ibis::table.

\subsubsection{3 virtual int ibis::tabula::dump (std::ostream \&, const char $*$ ) const [inline, virtual]}

Dump the values in ASCII form to the specified output stream.

The default delimiter is coma (","), which produces Comma-Separated-Values (CSV).

Implements ibis::table.

3.103.2.4 void ibis::tabula::estimate (const char $*$ cond, uint64_t \& nmin, uint64_t \& nmax) const [ i n ] i ne, virtual]

Estimate the number of rows satisfying the selection conditions.

The number of rows is between [nmin, nmax].

Implements ibis::table.

3.103.2.5 virtual int64_t ibis::tabula::getColumnAsBytes (const char *, char *) const [in]ine, virtual]

Retrieve all values of the named column. 
The member functions of this class only support access to whole column at a time. Use table: : cursor class for row-wise accesses. For fixed-width data types, the raw pointers are used to point to the values to be returned. In these cases, the caller is responsible for allocating enough storage for the values to be returned.

Implements ibis::table.

3.103.2.6 virtual int64_t ibis::tabula::getColumnAsDoubles (const char $*$, double $*$ ) const [inline, virtual]

Retrieve all values of the named column.

The member functions of this class only support access to whole column at a time. Use table: : cursor class for row-wise accesses. For fixed-width data types, the raw pointers are used to point to the values to be returned. In these cases, the caller is responsible for allocating enough storage for the values to be returned.

Implements ibis::table.

3.103.2.7 virtual int64_t ibis::tabula::getColumnAsFloats (const char $*$, float $*$ ) const [in]ine, virtual]

Retrieve all values of the named column.

The member functions of this class only support access to whole column at a time. Use table: : cursor class for row-wise accesses. For fixed-width data types, the raw pointers are used to point to the values to be returned. In these cases, the caller is responsible for allocating enough storage for the values to be returned.

Implements ibis::table.

3.103.2.8 virtual int64_t ibis::tabula::getColumnAsInts (const char $*$, int32_t $*$ ) const [inline, virtual]

Retrieve all values of the named column.

The member functions of this class only support access to whole column at a time. Use table: : cursor class for row-wise accesses. For fixed-width data types, the raw pointers are used to point to the values to be returned. In these cases, the caller is responsible for allocating enough storage for the values to be returned.

Implements ibis::table.

3.103.2.9 virtual int64_t ibis::tabula::getColumnAsLongs (const char $*$, int64_t $*$ ) const [inline, virtual]

Retrieve all values of the named column.

The member functions of this class only support access to whole column at a time. Use table: : cursor class for row-wise accesses. For fixed-width data types, the raw pointers are used to point to the values to be returned. In these cases, the caller is responsible for allocating enough storage for the values to be returned.

Implements ibis::table.

3.103.2.10 virtual int64_t ibis::tabula::getColumnAsShorts (const char $*$, int16_t $*$ ) const [inline, virtual]

Retrieve all values of the named column.

The member functions of this class only support access to whole column at a time. Use table: : cursor class for row-wise accesses. For fixed-width data types, the raw pointers are used to point to the values to be returned. In these cases, the caller is responsible for allocating enough storage for the values to be returned.

Implements ibis::table. 
3.103.2.11 virtual int64_t ibis::tabula::getColumnAsStrings (const char $*$, std::vector $<$ std::string $>\&$ ) const [inline, virtual]

Retrieve the null-terminated strings as a vector of std::string objects.

Both ibis::CATEGORY and ibis::TEXT types can be retrieved using this function.

Implements ibis::table.

3.103.2.12 virtual int64_t ibis::tabula::getColumnAsUBytes (const char $*$, unsigned char $*$ ) const [inline, virtual]

Retrieve all values of the named column.

The member functions of this class only support access to whole column at a time. Use table: : cursor class for row-wise accesses. For fixed-width data types, the raw pointers are used to point to the values to be returned. In these cases, the caller is responsible for allocating enough storage for the values to be returned.

Implements ibis::table.

3.103.2.13 virtual int64_t ibis::tabula::getColumnAsUInts (const char $*$, uint32_t $*$ ) const [in]ine, virtual]

Retrieve all values of the named column.

The member functions of this class only support access to whole column at a time. Use table: : cursor class for row-wise accesses. For fixed-width data types, the raw pointers are used to point to the values to be returned. In these cases, the caller is responsible for allocating enough storage for the values to be returned.

Implements ibis::table.

3.103.2.14 virtual int64_t ibis::tabula::getColumnAsULongs (const char $*$, uint64_t $*$ ) const [in]ine, virtual]

Retrieve all values of the named column.

The member functions of this class only support access to whole column at a time. Use table: : cursor class for row-wise accesses. For fixed-width data types, the raw pointers are used to point to the values to be returned. In these cases, the caller is responsible for allocating enough storage for the values to be returned.

Implements ibis::table.

3.103.2.15 virtual int64_t ibis::tabula::getColumnAsUShorts (const char $*$, uint16_t $*$ ) const [in]ine, virtual]

Retrieve all values of the named column.

The member functions of this class only support access to whole column at a time. Use table: : cursor class for row-wise accesses. For fixed-width data types, the raw pointers are used to point to the values to be returned. In these cases, the caller is responsible for allocating enough storage for the values to be returned.

Implements ibis::table.

3.103.2.16 virtual long ibis::tabula::getHistogram (const char $*$, const char $*$, double, double, double, std::vector $<$ size_t $>$ \&) const [inline, virtual]

Compute the histogram of the named column.

This version uses the user specified bins: [begin, begin+stride) [begin+stride, begin $+2 *$ stride), .... A record is placed in bin

( $\mathrm{x}$ - begin) / stride, where the first bin is bin 0 . This gives a total of

(end - begin) / stride bins. 
Note:

Records (rows) outside of the range [begin, end] are not counted.

Non-positive stride is considered as an error.

If end is less than begin, an empty array counts is returned along with return value 0 .

Implements ibis::table.

3.103.2.17 virtual long ibis::tabula::getHistogram2D (const char $*$, const char $*$, double, double, double, const char $*$, double, double, double, std::vector $<$ size_t $>\boldsymbol{\&}$ ) const [inline, virtual]

Compute a two-dimension histogram on columns cname1 and name2.

The bins along each dimension are defined the same way as in function getHistogram. The array counts stores the two-dimensional bins with the first dimension as the slow varying dimension following $\mathrm{C}$ convention for ordering multi-dimensional arrays.

Implements ibis::table.

3.103.2.18 virtual long ibis::tabula::getHistogram3D (const char $*$, const char $*$, double, double, double, const char $*$, double, double, double, const char $*$, double, double, double, std::vector $<$ size_t $>\&$ ) const [inline, virtual]

Compute a three-dimensional histogram on the named columns.

The triplets $<$ begin, end, stride $>$ are used the same ways in getHistogram and getHistogram2D. The three dimensional bins are linearized in count $s$ with the first being the slowest varying dimension and the third being the fastest varying dimension following the $\mathrm{C}$ convention for ordering multi-dimensional arrays.

Implements ibis::table.

3.103.2.19 virtual table $*$ ibis::tabula::groupby (const stringList \&) const [inline, virtual]

Perform aggregate functions on the current table.

It produces a new table. The list of strings passed to this function are interpreted as a set of names followed by a set of functions. Currently, only functions COUNT, AVG, MIN, MAX, and SUM are supported.

Implements ibis::table.

\subsubsection{0 virtual void ibis::tabula::indexSpec (const char $*$, const char $*$ ) [inline, virtual]}

Replace the current indexing option.

If no column name is specified, it resets the indexing option for the table.

Implements ibis::table.

\subsubsection{1 virtual const char* ibis::tabula::indexSpec (const char $*$ ) const [inline, virtual ]}

Retrieve the current indexing option.

If no column name is specified, it retrieve the indexing option for the table.

Implements ibis::table.

\subsubsection{2 virtual void ibis::tabula::orderby (const stringList \&) [inline, virtual]}

Reorder the rows.

Sort the rows in ascending order of the columns specified in the list of column names. This function is not designated const because though it does not change the content in SQL logic, but it may change internal representations. 


\section{Note:}

If an empty list is passed to this function, it will reorder rows using all columns with the column having the smallest number of distinct values first.

Implements ibis::table.

The documentation for this class was generated from the following file:

- tab.h

\subsection{4 ibis::tafel Class Reference}

An expandable table.

\#include <tafel.h>

Inheritance diagram for ibis::tafel::

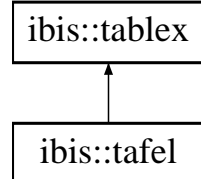

\section{Public Member Functions}

- virtual int addColumn (const char $*$ cname, ibis::TYPE_T ctype, const char $*$ cdesc)

- virtual int addColumn (const char $*$ cname, ibis::TYPE_T ctype) Add a column.

- virtual int append (const char $*$ cname, uint64_t begin, uint64_t end, void $*$ values) Add values to the named column.

- virtual int appendRow (const char $*$, const char $*$ ) Append a row stored in ASCII form.

- virtual int appendRow (const ibis::table::row \&) Add one row.

- virtual int appendRows (const std::vector $<$ ibis::table::row $>\&$ ) Add multiple rows.

- virtual int readCSV (const char $*$ filename, const char $*$ delimiters) Read the content of the specified as comma-separated values.

- virtual int write (const char $*$ dir, const char $*$ tname, const char $*$ tdesc) const Write the in-memory data records to the specified directory on disk.

\section{Protected Types}

- typedef std::map $<$ const char $*$, column $*$, ibis::lessi $>$ columnList 


\section{Protected Member Functions}

- template $<$ typename $\mathrm{T}>$ void append (const std::vector $<$ std::string $>$ \&nm, const std::vector $<\mathrm{T}>$ \&va, std::vector $<$ array_t $<\mathrm{T}>*>$ \&buf)

- template $<$ typename $\mathrm{T}>$ void append (const $\mathrm{T} *$ in, ibis::bitvector::word_t be, ibis::bitvector::word_t en, array_$\mathrm{t}<\mathrm{T}>$ \&out, const $\mathrm{T}$ \& fill, ibis::bitvector \&mask) const

- void appendString (const std::vector $<$ std::string $>\& n m$, const std::vector $<$ std::string $>\&$ va, std::vector $<$ std::vector $<$ std::string $>*>$ \&buf)

- void appendString (const std::vector $<$ std::string $>*$ in, ibis::bitvector::word_t be, ibis::bitvector::word_t en, std::vector $<$ std::string $>$ \&out, ibis::bitvector \&mask) const

- void clear ()

Clear the content of the buffers.

- template $<$ typename $\mathrm{T}>$ void locate (ibis::TYPE_T, std::vector $<$ array_t $<\mathrm{T}>*>$ \&buf) const

- void locateString (ibis::TYPE_T t, std::vector $<$ std::vector $<$ std::string $>*>$ \&buf) const

- void normalize ()

Make all short columns catch up with the longest one.

- int parseLine (const char $*$ str, const char $*$ del, const char $* \mathrm{id}$ )

- template $<$ typename $\mathrm{T}>$ int writeColumn (int fdes, ibis::bitvector::word_t nold, ibis::bitvector::word_t nnew, const array_t $<\mathrm{T}>$ \&vals, const $\mathrm{T}$ \& fill, ibis::bitvector \&totmask, const ibis::bitvector \&newmask) const

- int writeString (int fdes, ibis::bitvector::word_t nold, ibis::bitvector::word_t nnew, const std::vector < std::string $>$ \&vals, ibis::bitvector \&totmask, const ibis::bitvector \&newmask) const

\section{Protected Attributes}

- std::vector $<$ column $*>$ colorder

Order of columns as they were specified through addColumn.

- columnList cols

List of columns in alphabetical order.

- ibis::bitvector::word_t nrows

Number of rows of this table.

\section{Classes}

- struct column

\subsubsection{Detailed Description}

An expandable table.

It inherents from ibis::tablex only therefore does not support any querying functions.

Note:

The word tafel is a German word for table. 


\subsubsection{Member Function Documentation}

3.104.2.1 int ibis::tafel::append (const char $*$ cname, uint64_t begin, uint64_t end, void * values) [virtual]

Add values to the named column.

The column name must be in the table already. The first value is to be placed at row begin (the row numbers start with 0 ) and the last value before row end. The array values must contain values of the correct type corresponding to the type specified before.

\section{Note:}

Since each column may have different number of rows filled, the number of rows in the table is considered to be the maximum number of rows filled of all columns.

This function can not be used to introduce new columns in a table. A new column must be added with addcolumn.

appendRow

Implements ibis::tablex.

\subsubsection{2 int ibis::tafel::appendRow (const char $*$, const char $*$ ) [virtual]}

Append a row stored in ASCII form.

The ASCII form of the values are assumed to be separated by comma (,) or space, but additional delimiters may be added through the second argument.

Implements ibis::tablex.

\subsubsection{3 int ibis::tafel::appendRow (const ibis::table::row \&) [virtual]}

Add one row.

If an array of names has the same number of elements as the array of values, the names are used as column names. If the names are not specified explicitly, the values are assigned to the columns of the same data type in the order as they are specified through addColumn or if the same order as they are recreated from an existing dataset (which is typically alphabetical).

\section{Note:}

The column names are not case-sensitive.

Like append, this function can not be used to introduce new columns in a table. A new column must be added with addColumn.

Since the various columns may have different numbers of rows filled, the number of rows in the table is assumed to the largest number of rows filled so far. The new row appended here increases the number of rows in the table by 1 . The unfilled rows are assumed to be null.

A null value of an integer column is recorded as the maximum possible of the type of integer. A null value of a floating-point valued column is recorded as a quiet $\mathrm{NaN}$ (Not-a-Number). A null value of a string-valued column is recorded as an empty string. In all cases, a null mask is used to indicate that they are null values.

Implements ibis::tablex.

\subsubsection{4 int ibis::tafel::appendRows (const std::vector $<$ ibis::table::row $>\&$ \&) [virtual]}

Add multiple rows.

Rows in the incoming vector are processed on after another. The ordering of the values in earlier rows are automatically carried over to the later rows until another set of names is specified. appendRow

Implements ibis::tablex. 


\subsubsection{5 int ibis::tafel::readCSV (const char $*$ filename, const char $*$ delimiters) $\quad$ [ virtual ]}

Read the content of the specified as comma-separated values.

Append the records to this table. By default the records are delimited by comma (,) and blank space. One may specify additional delimiters using the second argument.

Implements ibis::tablex.

\subsubsection{6 int ibis::tafel::write (const char $*$ dir, const char $*$ tname, const char $*$ tdesc) const [virtual]}

Write the in-memory data records to the specified directory on disk.

If the table name (tname) is a null string or an empty, the last component of the directory name is used. If the description (tdesc) is a null string or an empty string, a time stamp will be printed in its place. If the specified directory already contains data, the new records will be appended to the existing data. In this case, the table name specified here will overwrite the existing name, but the existing name and description will be retained if the current arguments are null strings or empty strings. The data type associated with this table will overwrite the existing data type information.

Implements ibis::tablex.

The documentation for this class was generated from the following files:

- tafel.h

- tafel.cpp

\subsection{5 ibis::text Class Reference}

A minimalistic structure for storing arbitrary text fields.

\#include <category.h>

Inheritance diagram for ibis::text::

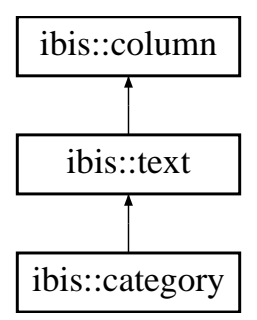

\section{Public Member Functions}

- virtual long append (const char $* \mathrm{dt}$, const char $* \mathrm{df}$, const uint32_t nold, const uint32_t nnew, const uint32_t nbuf, char $*$ buf)

Append the data file stored in directory $\mathrm{df}$ to the corresponding data file in directory $\mathrm{dt}$.

- virtual double estimateCost (const ibis::qMultiString \&cmp) const

- virtual double estimateCost (const ibis::qString \&cmp) const

- virtual const char $*$ findString (const char $*$ str) const

If the input string is found in the data file, it is returned, else this function returns 0.

- virtual void getString (uint32_t i, std::string \&val) const

- const column $*$ IDColumnForKeywordIndex () const

- virtual long keywordSearch (const char $*$ str) const 
- virtual long keywordSearch (const char $*$ str, ibis::bitvector \&hits) const

- virtual void print (std::ostream \&out) const

- virtual long search (const std::vector < std::string $>$ \&strs) const

- virtual long search (const char $*$ str) const

- virtual long search (const std::vector $<$ std::string $>$ \&strs, ibis::bitvector \&hits) const

Given a group of string literals, return a bitvector that matches anyone of the input strings.

- virtual long search (const char $*$ str, ibis::bitvector \&hits) const

Given a string literal, return a bitvector that marks the strings that matches it.

- virtual std::vector $<$ std::string $>*$ selectStrings (const bitvector \&mask) const

- virtual array_t $<$ uint32_t $>*$ selectUInts (const bitvector \&mask) const

Return the integer values of the records marked 1 in the mask.

- text (const ibis::column \&col)

- text (const part *tbl, const char *name, ibis::TYPE_T t=ibis::TEXT)

- text (const part $*$ tbl, FILE $*$ file)

- virtual void write (FILE $*$ file) const

Write the current content to the TDC file.

\section{Protected Member Functions}

- int readString (std::string \&, int, long, long, char $*$, uint32_t, uint32_t \&, off_t \&) const

Read on string from an open file.

- void readString (uint32_t i, std::string \&val) const

Return the ith string, i.e., the string in the ith row/record.

- void startPositions (const char $*$ dir, uint32_t nbuf, char $*$ buf) const

Locate the starting position of each string and write the positions as unsigned integers to a file with .sp as extension.

\subsubsection{Detailed Description}

A minimalistic structure for storing arbitrary text fields.

The keyword search operation is implemented through a boolean term-document matrix (ibis: : keywords) that is actually generated externally.

\subsubsection{Member Function Documentation}

3.105.2.1 long ibis::text::append (const char $* d t$, const char $* d f$, const uint32_t nold, const uint32_t nnew, const uint32_t nbuf, char $*$ buf) [virtual]

Append the data file stored in directory $\mathrm{df}$ to the corresponding data file in directory $\mathrm{dt}$.

Use the buffer buf to copy data in large chuncks.

Note:

No error checking is performed.

Does not check for missing entries. May cuase records to be misaligned.

Reimplemented from ibis::column.

Reimplemented in ibis::category. 


\subsubsection{2 const char $*$ ibis::text::findString (const char $*$ str) const $\quad$ [ virtua 1$]$}

If the input string is found in the data file, it is returned, else this function returns 0.

It needs to keep both the data file and the starting position file open at the same time.

Reimplemented from ibis::column.

3.105.2.3 int ibis::text::readString (std::string \& res, int fdes, long be, long en, char * buf, uint32_t nbuf, uint32_t \& inbuf, off_t \& boffset) const [ protected]

Read on string from an open file.

The string starts at position be and ends at en. The content may be in the array buf.

\subsubsection{4 void ibis::text::readString (uint32_t $\boldsymbol{i}$, std::string \& ret) const [protected]}

Return the ith string, i.e., the string in the ith row/record.

It goes through a two-stage process by reading from two files. This is quite slow!

\subsubsection{5 array_t $<$ uint32_t $>*$ ibis::text::selectUInts (const bitvector \& mask) const [ virtual]}

Return the integer values of the records marked 1 in the mask.

This indicates to ibis::bundle that every string value is distinct. It also forces the sorting procedure to produce an order following the order of the entries in the table. This makes the print out of an ibis::text field quite less useful than others!

Reimplemented from ibis::column.

Reimplemented in ibis::category.

\subsubsection{6 void ibis::text::startPositions (const char $* \boldsymbol{d i r}$, uint32_t $\boldsymbol{n} \boldsymbol{b} \boldsymbol{u} \boldsymbol{f}$, char $* \boldsymbol{b} \boldsymbol{u} \boldsymbol{f}$ ) const [protected]}

Locate the starting position of each string and write the positions as unsigned integers to a file with .sp as extension.

If $\mathrm{dir}$ is a nil pointer, the directory is default to the current working directory of the data table. Arguments nbuf and buf are used as temporary working space. If nbuf $=0$, this function allocates its own working space.

The documentation for this class was generated from the following files:

- category.h

- category.cpp

\subsection{6 ibis::uniformRandomNumber Class Reference}

A functor to generate uniform random number in the range $[0,1)$.

\#include <twister.h>

Inheritance diagram for ibis::uniformRandomNumber::

\begin{tabular}{|c|}
\hline ibis::uniformRandomNumber \\
\hline ibis::MersenneTwister \\
\hline
\end{tabular}




\section{Public Member Functions}

- virtual double operator ()()$=0$

\subsubsection{Detailed Description}

A functor to generate uniform random number in the range $[0,1)$.

The documentation for this class was generated from the following file:

- twister.h

\subsection{7 ibis::util::counter Class Reference}

A simple global counter.

\#include <util.h>

\section{Public Member Functions}

- counter (const char $*$ m="ibis::util::counter")

- uint32_t operator() ()

Return the current count and increment the count.

- void reset ()

Reset count to zero.

- uint32_t value () const

Return the current count value.

\subsubsection{Detailed Description}

A simple global counter.

Each time the operator() is called, it is incremented by 1. Calls from different threads are serialized through a mutual exclusion lock.

The documentation for this class was generated from the following file:

- util.h

\subsection{8 ibis::util::ioLock Class Reference}

A global IO mutex lock.

\#include <util.h>

\subsubsection{Detailed Description}

A global IO mutex lock.

The documentation for this class was generated from the following files:

- util.h

- util.cpp 


\subsection{9 ibis::util::mutexLock Class Reference}

An wrapper class for perform pthread_mutex_lock/unlock.

\#include <util.h>

Public Member Functions

- mutexLock (pthread_mutex_t $* \mathrm{lk}$, const char $* \mathrm{~m}$ )

\subsubsection{Detailed Description}

An wrapper class for perform pthread_mutex_lock/unlock.

The documentation for this class was generated from the following file:

- util.h

\subsection{0 ibis::util::quietLock Class Reference}

An wrapper class for perform pthread_mutex_lock/unlock.

\#include <util.h>

Public Member Functions

- quietLock (pthread_mutex_t $* 1 \mathrm{lk}$, const char $* \mathrm{~m})$

\subsubsection{Detailed Description}

An wrapper class for perform pthread_mutex_lock/unlock.

Avoid invoking ibis::util::logMessage so it can be used inside ibis::util::logMessage.

The documentation for this class was generated from the following file:

- util.h

\subsection{1 ibis::util::readLock Class Reference}

An wrapper class for perform pthread_rwlock_rdlock/unlock.

\#include <util.h>

\section{Public Member Functions}

- readLock (pthread_rwlock_t $* 1 \mathrm{k}$, const char $* \mathrm{~m})$

\subsubsection{Detailed Description}

An wrapper class for perform pthread_rwlock_rdlock/unlock.

The documentation for this class was generated from the following file:

- util.h 


\subsection{2 ibis::util::writeLock Class Reference}

An wrapper class for perform pthread_rwlock_wrlock/unlock.

\#include <util.h>

\section{Public Member Functions}

- writeLock (pthread_rwlock_t $* \mathrm{lk}$, const char $* \mathrm{~m})$

\subsubsection{Detailed Description}

An wrapper class for perform pthread_rwlock_wrlock/unlock.

The documentation for this class was generated from the following file:

- util.h

\subsection{3 ibis::zona Class Reference}

The two-level equality-equality code.

\#include <irelic.h>

Inheritance diagram for ibis::zona::

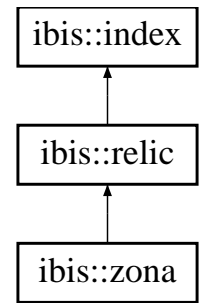

\section{Public Member Functions}

- virtual long append (const char $* \mathrm{dt}$, const char $* \mathrm{df}$, uint32_t nnew)

Extend the index.

- virtual uint32_t estimate (const ibis::qContinuousRange \&expr) const Returns an upper bound on the number of hits.

- virtual double estimateCost (const ibis::qContinuousRange \&expr) const Estimate the code of evaluate a range condition.

- virtual long evaluate (const ibis::qContinuousRange \&expr, ibis::bitvector \&hits) const To evaluate the exact hits.

- virtual const char $*$ name () const

Returns the name of the index, similar to the function type, but returns a string instead.

- virtual void print (std::ostream \&out) const

Prints human readable information. 
- virtual void read (ibis::fileManager::storage $*$ st)

Reconstructs an index from an array of bytes.

- virtual void read (const char $*$ idxfile)

Reconstructs an index from the named file.

- virtual INDEX_TYPE type () const

Returns an index type identifier.

- virtual void write (const char $* \mathrm{dt})$ const

Save index to a file.

- zona (const ibis::column $*$ c, ibis::fileManager::storage $*$ st, uint32_t offset=8)

The leading portion of the index file is the same as ibis::relic, which allows the constructor of the base class to work properly.

- zona (const ibis::column $* \mathrm{c}=0$, const char $* \mathrm{f}=0$ )

\section{Protected Member Functions}

- virtual void clear ()

Clear the existing content.

\subsubsection{Detailed Description}

The two-level equality-equality code.

Note:

zone is Italian word for zone, the name of the binned version of the two-level equality-equality code.

\subsubsection{Constructor \& Destructor Documentation}

\subsubsection{1 ibis::zona::zona (const ibis::column $* c$, ibis::fileManager::storage $* s t$, uint32_t start $=8$ )}

The leading portion of the index file is the same as ibis::relic, which allows the constructor of the base class to work properly.

The content following the last bitvector in ibis::relic is as follows, writeCoarse.

nc (uint32_t) - number of coarse bins. cbounds (unsigned[nc+1]) - boundaries of the coarse bins. coffsets(int32_$\mathrm{t}[\mathrm{nc}+1])$ - starting position of the coarse level bitmaps. cbits (bitvector[nc]) - bitvector laid out one after another.

\subsubsection{Member Function Documentation}

3.113.3.1 long ibis::zona::evaluate (const ibis::qContinuousRange \& expr, ibis::bitvector \& hits) const [virtual]

To evaluate the exact hits.

On success, return the number of hits, otherwise a negative value is returned.

Reimplemented from ibis::relic. 


\subsubsection{2 void ibis::zona::print (std::ostream \& out) const [virtual]}

Prints human readable information.

Outputs information about the index as text to the specified output stream.

Reimplemented from ibis::relic.

\subsubsection{3 void ibis::zona::read (ibis::fileManager::storage $*$ st) [virtual]}

Reconstructs an index from an array of bytes.

Intended for internal use only!

Reimplemented from ibis::relic.

\subsubsection{4 void ibis::zona::read (const char $*$ idxfile) $\quad$ [virtual]}

Reconstructs an index from the named file.

The name can be the directory containing an index file. In this case, the name of the index file must be the name of the column followed by ".idx" suffix.

Reimplemented from ibis::relic.

\subsubsection{5 void ibis::zona::write (const char $* \boldsymbol{d} t)$ const $\quad$ [virtual]}

Save index to a file.

Outputs the index in a compact binary format to the named file or directory. The index file contains a header that can be identified by the function isIndex.

Reimplemented from ibis::relic.

The documentation for this class was generated from the following files:

- irelic.h

- ixzona.cpp

\subsection{4 ibis::zone Class Reference}

A two-level index.

\#include <ibin.h>

Inheritance diagram for ibis::zone::

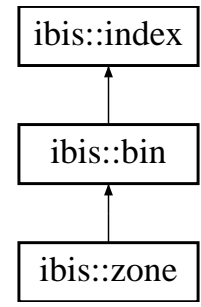

\section{Public Member Functions}

- virtual void adjustLength (uint32_t nrows)

- long append (const ibis::zone \&tail)

- virtual long append (const char $* \mathrm{dt}$, const char $* \mathrm{df}$, uint32_t nnew) 
Extend the index.

- virtual void binBoundaries (std::vector $<$ double $>\&$ ) const

The function binBoundaries and binWeights return bin boundaries and counts of each bin respectively.

- virtual void binWeights (std::vector $<$ uint32_t $>$ \&) const

- virtual void estimate (const ibis::qContinuousRange \&expr, ibis::bitvector \&lower, ibis::bitvector \&upper) const

Computes an approximation of hits as a pair of lower and upper bounds.

- virtual long evaluate (const ibis::qContinuousRange \&expr, ibis::bitvector \&hits) const

To evaluate the exact hits.

- virtual const char $*$ name () const

Returns the name of the index, similar to the function type, but returns a string instead.

- virtual uint32_t numBins () const

- virtual void print (std::ostream \&out) const

Prints human readable information.

- virtual void read (ibis::fileManager::storage $*$ st)

Reconstructs an index from an array of bytes.

- virtual void read (const char $*$ idxfile)

Reconstructs an index from the named file.

- virtual void speedTest (std::ostream \&out) const

Time some logical operations and print out their speed.

- virtual INDEX_TYPE type () const

Returns an index type identifier.

- virtual float undecidable (const ibis::qContinuousRange \&expr, ibis::bitvector \&iffy) const

Mark the position of the rows that can not be decided with this index.

- virtual void write (const char $* \mathrm{dt}$ ) const

Save index to a file.

- zone (const ibis::bin \&rhs)

- zone (const ibis::column $*$ c, ibis::fileManager::storage $*$ st, uint32_t offset=8)

\subsubsection{Detailed Description}

A two-level index.

Both levels are not cumulative, i.e., both levels are equality encoded.

\subsubsection{Member Function Documentation}

3.114.2.1 void ibis::zone::estimate (const ibis::qContinuousRange \& expr, ibis::bitvector \& lower, ibis::bitvector \& upper) const [virtual]

Computes an approximation of hits as a pair of lower and upper bounds. 


\section{Parameters:}

expr the query expression to be evaluated.

lower a bitvector marking a subset of the hits. All rows marked with one (1) are definitely hits.

upper a bitvector marking a superset of the hits. All hits are marked with one, but some of the rows marked one may not be hits. If the variable upper is empty, the variable lower is assumed to contain the exact answer.

Reimplemented from ibis::bin.

3.114.2.2 long ibis::zone::evaluate (const ibis::qContinuousRange \& expr, ibis::bitvector \& hits) const [virtual]

To evaluate the exact hits.

On success, return the number of hits, otherwise a negative value is returned.

Reimplemented from ibis::bin.

\subsubsection{3 void ibis::zone::print (std::ostream \& out) const [virtual]}

Prints human readable information.

Outputs information about the index as text to the specified output stream.

Reimplemented from ibis::bin.

3.114.2.4 void ibis::zone::read (ibis::fileManager::storage $* \boldsymbol{s t}$ ) [virtual]

Reconstructs an index from an array of bytes.

Intended for internal use only!

Reimplemented from ibis::bin.

\subsubsection{5 void ibis::zone::read (const char $*$ idxfile) $\quad$ [ virtual]}

Reconstructs an index from the named file.

The name can be the directory containing an index file. In this case, the name of the index file must be the name of the column followed by ".idx" suffix.

Reimplemented from ibis::bin.

3.114.2.6 float ibis::zone::undecidable (const ibis::qContinuousRange \& expr, ibis::bitvector \& iffy) const [virtual]

Mark the position of the rows that can not be decided with this index.

\section{Parameters:}

expr the range conditions to be evaluated.

iffy the bitvector marking the positions of rows that can not be decided using the index. Return value is the expected fraction of undecided rows that might satisfy the range conditions.

Reimplemented from ibis::bin.

3.114.2.7 void ibis::zone::write (const char $* \boldsymbol{d t})$ const [ virtual]

Save index to a file. 
Outputs the index in a compact binary format to the named file or directory. The index file contains a header that can be identified by the function isIndex.

Reimplemented from ibis::bin.

The documentation for this class was generated from the following files:

- ibin.h

- ixzone.cpp

\section{FastBit File Documentation}

\section{1 array_t.h File Reference}

Definition of template array_t.

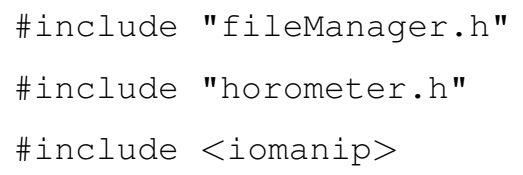

\section{Namespaces}

- namespace ibis

- namespace ibis::util

\section{Classes}

- class array_t $\mathrm{t}<\mathrm{T}>$

Template array_t implements a replacement of std::vector.

\section{Functions}

- template $<$ class $\mathrm{T}>$ void ibis: :util::reorder (array_t $<\mathrm{T} *>$ \&arr, const array_t $<$ uint32_t $>$ \&ind)

- template $<$ class $\mathrm{T}>$ void ibis::util::reorder (array_t $<\mathrm{T}>$ \&arr, const array_t $<$ uint32_t $>$ \&ind)

\subsubsection{Detailed Description}

Definition of template array_t.

Note:

array_t is not in the name space ibis because the compilers used during the early development of this project did not accept templates inside a name space.

\section{2 bitvector.h File Reference}

Definition of Word-Aligned Hybrid code.

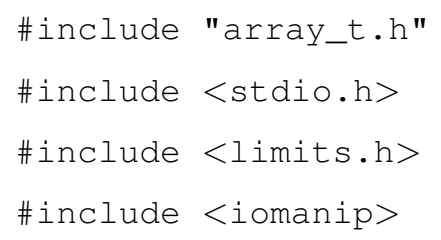




\section{Classes}

- class ibis::bitvector

A data structure to represent a sequence of bits.

- struct ibis::bitvector::active_word

The struct active_word stores the last few bits that do not fill a whole word.

- class ibis::bitvector::const_iterator

The const_iterator class. It iterates on the individual bits.

- class ibis::bitvector::indexSet

The indexSet stores positions of bits that are one.

- class ibis::bitvector::iterator

The iterator that allows modification of bits.

- struct ibis::bitvector::run

An internal struct used during logical operations to track the usage of fill words.

\section{Functions}

- std::ostream \& operator $<<($ std::ostream \&, const ibis::bitvector \&)

\subsubsection{Detailed Description}

Definition of Word-Aligned Hybrid code.

\section{3 bitvector64.h File Reference}

Definition of 64-bit version of the Word-Aligned Hybrid code.

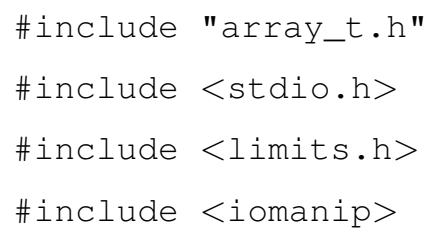

\section{Classes}

- class ibis::bitvector64

A data structure to represent a sequence of bits.

- struct ibis::bitvector64::active_word

The struct active_word stores the last few bits that do not fill a whole word.

- class ibis::bitvector64::const_iterator

The const_iterator class. It iterates on the individual bits.

- class ibis::bitvector64::indexSet

The indexSet stores positions of bits that are one. 
- class ibis::bitvector64::iterator

The iterator that allows modification of bits.

- struct ibis::bitvector64::run

An internal struct used during logical operations to track the usage of fill words.

\section{Functions}

- std::ostream \& operator $<<($ std::ostream \&, const ibis::bitvector64 \&)

\subsubsection{Detailed Description}

Definition of 64-bit version of the Word-Aligned Hybrid code.

\section{4 bord.h File Reference}

Defines ibis: : bord.

\#include "table.h"

\#include "util.h"

\#include "part.h"

\section{Namespaces}

- namespace ibis

\section{Classes}

- class ibis::bord

Class ibis::bord stores all its data in memory.

- class ibis::bord::column

An in-memory version of ibis::column.

- class ibis::bord::cursor

- struct ibis::bord::cursor::bufferElement

- class ibis::bord::part

\subsubsection{Detailed Description}

Defines ibis : : bord.

This is an in-memory table, which a single data partition completely residing in memory.

\section{5 bundle.h File Reference}

Designed to store selected values.

\#include "util.h" 


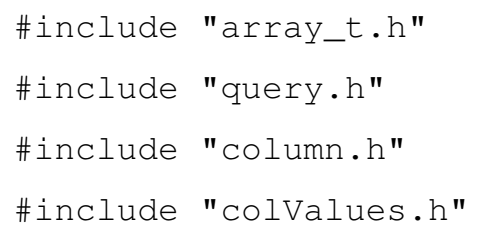

\section{Namespaces}

- namespace ibis

\section{Classes}

- class ibis::bundle

The public interface of bundles.

- class ibis::bundle0

The null bundle. It contains only a list of RIDs.

- class ibis::bundle1

The bundle with only one component.

- class ibis::bundles

The bundle with multiple components.

- class ibis::query::result

The class ibis::query::result allows user to retrieve query result one row at a time.

\subsubsection{Detailed Description}

Designed to store selected values.

The class ibis::bundle is use to represent a sorted version of the selected columns of a query. The selected columns can be of any type. The string values are internally recorded as integers. The bundles are written to a directory containing other type of information about the query.

This is an incore implementation, that is, it stores all relevant values in memory. It is intended to be used only to sort the selected values and immediately write out the content to files.

When multiple components are selected, a generic version of the sorting algorithm is used. It should be faster to handle special versions separately. For example, if all the selected components are of the same type, it is possible to use a more compact array structure for comparisons. It might be also useful to separate out the case where there are only two components.

\section{6 capi.h File Reference}

This header file defines a C API for accessing functions of FastBit IBIS implementations.

\section{Typedefs}

- typedef FastBitQuery $*$ FastBitQueryHandle

A handle to use used by C client.

- typedef FastBitResultSet $*$ FastBitResultSetHandle 


\section{Functions}

- int fastbit_build_index (const char $*$ indexLocation, const char $*$ cname, const char $*$ indexOptions)

Build an index for the named attribute.

- int fastbit_build_indexes (const char $*$ indexLocation)

Build indices for all attributes in the named directory.

- FastBitQueryHandle fastbit_build_query (const char $*$ selectClause, const char $*$ indexLocation, const char $*$ queryConditions)

Build a new FastBit query.

- FastBitResultSetHandle fastbit_build_result_set (FastBitQueryHandle query)

Build a new result set from a query object.

- void fastbit_cleanup (void)

Clean up resources hold by FastBit file manager.

- int fastbit_destroy_query (FastBitQueryHandle query)

Free all resource associated with the handle.

- int fastbit_destroy_result_set (FastBitResultSetHandle rset)

Destroy a result set.

- const char * fastbit_get_from_clause (FastBitQueryHandle query)

Return the table name.

- const double $*$ fastbit_get_qualified_double (FastBitQueryHandle query, const char $*$ cname)

- const float $*$ fastbit_get_qualified_float (FastBitQueryHandle query, const char $*$ cname)

- const int32_t $*$ fastbit_get_qualified_int (FastBitQueryHandle query, const char $*$ cname)

Return a pointer to an array holding the values of attribute att that qualifies the specified selection conditions.

- const uint32_t $*$ fastbit_get_qualified_unsigned (FastBitQueryHandle query, const char $*$ cname)

- int fastbit_get_result_columns (FastBitQueryHandle query)

Count the number of columns selected in the select clause of the query.

- int fastbit_get_result_rows (FastBitQueryHandle query)

Return the number of hits in the query.

- const char * fastbit_get_select_clause (FastBitQueryHandle query)

Return the string form of the select clause.

- int fastbit_get_verbose_level (void)

Return the current verboseness level.

- const char $*$ fastbit_get_where_clause (FastBitQueryHandle query)

Return the where clause of the query.

- void fastbit_init (const char $*$ rcfile)

Initialization function.

- int fastbit_purge_index (const char *indexLocation, const char $*$ cname)

Purge the index of the named attribute. 
- int fastbit_purge_indexes (const char $*$ indexLocation)

Purge all index files.

- double fastbit_result_set_get_double (FastBitResultSetHandle rset, const char $*$ cname)

Get the value of the named column as a double-precision floating-point number.

- float fastbit_result_set_get_float (FastBitResultSetHandle rset, const char *cname)

Get the value of the named column as a single-precision floating-point number.

- int fastbit_result_set_get_int (FastBitResultSetHandle rset, const char $*$ cname)

Get the value of the named column as an integer.

- const char * fastbit_result_set_get_string (FastBitResultSetHandle rset, const char *cname)

Get the value of the named column as a string.

- unsigned fastbit_result_set_get_unsigned (FastBitResultSetHandle rset, const char $*$ cname)

Get the value of the named column as an unsigned integer.

- double fastbit_result_set_getDouble (FastBitResultSetHandle rset, unsigned position)

Get the value of the named column as a double-precision floating-point number.

- float fastbit_result_set_getFloat (FastBitResultSetHandle rset, unsigned position)

Get the value of the named column as a single-precision floating-point number.

- int32_t fastbit_result_set_getInt (FastBitResultSetHandle rset, unsigned position)

Get the value of the named column as an integer.

- const char * fastbit_result_set_getString (FastBitResultSetHandle rset, unsigned position)

Get the value of the named column as a string.

- uint32_t fastbit_result_set_getUnsigned (FastBitResultSetHandle rset, unsigned position)

Get the value of the named column as an unsigned integer.

- int fastbit_result_set_next (FastBitResultSetHandle rset)

Returns 0 if there are more results, otherwise returns -1 .

- int fastbit_set_verbose_level (int v)

Change the verboseness of FastBit functions.

\subsubsection{Detailed Description}

This header file defines a C API for accessing functions of FastBit IBIS implementations.

It deals with data tables as directories and queries as pointers to struct FastBitQuery.

Note:

For functions that return integer error code, 0 always indicate success, a negative number indicate error, a positive number may also be returned to carry results, such as in fastbit_get_result_size.

For functions that returns pointers, they may return a nil pointer in case of error. 


\subsubsection{Function Documentation}

4.6.2.1 FastBitQueryHandle fastbit_build_query (const char $*$ selectClause, const char $*$ indexLocation, const char $*$ queryConditions)

Build a new FastBit query.

This is logically equivalent to the SQL statement "SELECT selectClause FROM indexLocation WHERE queryConditions." A blank selectClause is equivalent to "count $(*)$ ".

Note:

Must call fastbit_destroy_query on the handle returned to free the resources.

\subsubsection{2 int fastbit_destroy_query (FastBitQueryHandle query)}

Free all resource associated with the handle.

Note:

The handle becomes invalid.

\subsubsection{3 const int32_t $*$ fastbit_get_qualified_int (FastBitQueryHandle query, const char $*$ cname)}

Return a pointer to an array holding the values of attribute att that qualifies the specified selection conditions.

Note:

The caller can NOT free the memory pointed by the pointer returned. Must call fastbit_destroy_query to free the memory after use. This applies to all other versions of fastbit_get_qualified_ttt.

\subsubsection{4 int fastbit_get_result_rows (FastBitQueryHandle query)}

Return the number of hits in the query.

It is also the number of rows in the result set. The arrays returned by gastbit_get_qualified_xxx shall have this many elements.

\subsubsection{5 void fastbit_init (const char $*$ rcfile)}

Initialization function.

May pass in a nil pointer if one is expected to use use the default configuartion files listed in the documentation of ibis::resources::read. One may call this function multiple times to read multiple configuration files to modify the parameters.

\subsubsection{6 int32_t fastbit_result_set_getInt (FastBitResultSetHandle rset, unsigned position)}

Get the value of the named column as an integer.

The argument index is the position (starting with 0 ) of the attribute in the select clause. This should be faster than the one with cname as argument since it avoids name look up.

\section{7 category.h File Reference}

Define two specialization of the column class.

\#include "irelic.h"

\#include "column.h" 


\section{Classes}

- class ibis::category

A specialized low-cardinality text field.

- class ibis::dictionary

Provide a mapping between strings and integers.

- class ibis::text

A minimalistic structure for storing arbitrary text fields.

\subsubsection{Detailed Description}

Define two specialization of the column class.

IBIS represents incoming data table with vertical partitioning. Each column object represents one column of the relational table. The terms used to describe each column of the table are strongly influenced by the first project using this software, a high-energy physics experiment named STAR.

\section{8 column.h File Reference}

Define the class column.

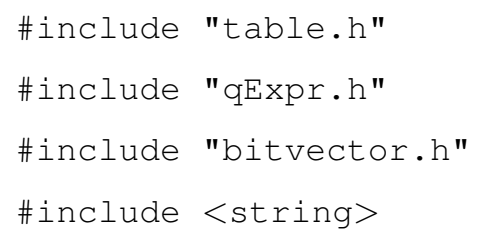

\section{Namespaces}

- namespace ibis

\section{Classes}

- class ibis::column

The class to represent a column of a data table.

- class ibis::column::indexLock

A class for controlling access of the index object of a column.

- class ibis::column::info

Some basic information about a column.

- class ibis::column::mutexLock

Provide a mutual exclusion lock on an ibis::column.

- class ibis::column::writeLock

Provide a write lock on a ibis:::column object. 


\section{Functions}

- std::ostream \& operator $<<$ (std::ostream \&out, const ibis::column \&prop)

\subsubsection{Detailed Description}

Define the class column.

A column of a relational table is also known as an attribute of a relation. In IBIS, columns are stored separate from each other. This storage strategy is commonly known as vertical partitioning.

\section{9 colValues.h File Reference}

A set of utility classes for storing the selected values.

\#include "column.h"

\section{Classes}

- class ibis::colDoubles

A class to store double precision floating-point values.

- class ibis::colFloats

A class to store single precision float-point values.

- class ibis::colInts

A class to store integer values.

- class ibis::colLongs

A class to store integer values.

- class ibis::colUInts

A class to store unsigned integer values.

- class ibis::colULongs

A class to store unsigned integer values.

- class ibis::colValues

A pure virtual base class.

\subsubsection{Detailed Description}

A set of utility classes for storing the selected values.

\subsection{0 const.h File Reference}

Defines common data types, constants and macros.

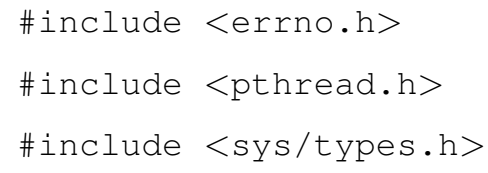




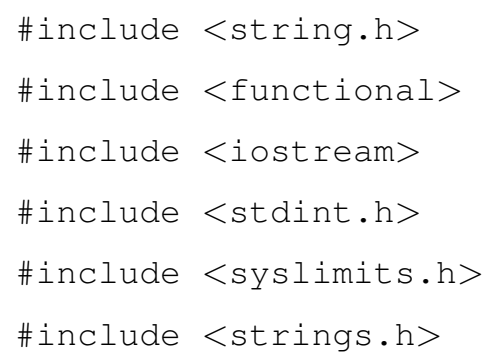

\section{Namespaces}

- namespace ibis

\section{Classes}

- struct_rwlock

- struct ibis::lessi

A case-insensitive version of less for comparing names of tables, columns, and resources.

- union ibis::rid_t

The object identifiers used to distinguish records.

- struct ibis::rid_t::name

As two 32-bit values.

\section{Defines}

- \#define DIRSEP $' r$

- \#define IBIS_REPLACEMENT_RWLOCK

- \#define int16_t short int

- \#define int32_t int

- \#define int64_t long long int

- \#define MAX_LINE 2048

- \#define MessageBox(x1, x2, x3, x4); \{\}

- \#define PATH_MAX 512

- \#define PREFERRED_BLOCK_SIZE 1048576

PREFERRED_BLOCK_SIZE is the parameter used to determine the logical page size during some I/O intensive operations, such as nested loop join.

- \#define REASON " " < < strerror(errno) $<<$ std::endl;

- \#define stricmp strcasecmp

- \#define strnicmp strncasecmp

- \#define THREAD_RWLOCK_INITIALIZER

- \#define TIME_BUF_LEN 32

- \#define uint16_t unsigned short int

- \#define uint32_t unsigned int

- \#define uint64_t unsigned long long int

\section{Typedefs}

- typedef_rwlock pthread_rwlock_t 


\section{Functions}

- int pthread_rwlock_destroy (pthread_rwlock_t $*$ rwlock)

- int pthread_rwlock_init (pthread_rwlock_t $*$ rwlock, void $*$ )

- int pthread_rwlock_rdlock (pthread_rwlock_t $*$ rwlock)

- int pthread_rwlock_tryrdlock (pthread_rwlock_t $*$ rwlock)

- int pthread_rwlock_trywrlock (pthread_rwlock_t $*$ rwlock)

- int pthread_rwlock_unlock (pthread_rwlock_t $*$ rwlock)

- int pthread_rwlock_wrlock (pthread_rwlock_t $*$ rwlock)

\section{Variables}

- int ibis::gVerbose

Verbosity level.

\subsubsection{Detailed Description}

Defines common data types, constants and macros.

Used by all files in the IBIS implementation of FastBit from the Scientific Data Management Research Group of Lawrence Berkeley National Laboratory.

\subsubsection{Define Documentation}

\subsubsection{1 \#define PREFERRED_BLOCK_SIZE 1048576}

PREFERRED_BLOCK_SIZE is the parameter used to determine the logical page size during some I/O intensive operations, such as nested loop join.

Many CPUs have 512KB cache, setting this value to 256K (262144) will allow about two such 'logical' block to be in cache at the same time, which should be good to things like nested loop join.

\subsubsection{2 \#define THREAD_RWLOCK_INITIALIZER}

Value:

\{PTHREAD_MUTEX_INITIALIZER, PTHREAD_COND_INITIALIZER, PTHREAD_COND_INITIALIZER, 0,0$\}$

\subsection{1 fileManager.h File Reference}

Defines a simple file Manager.

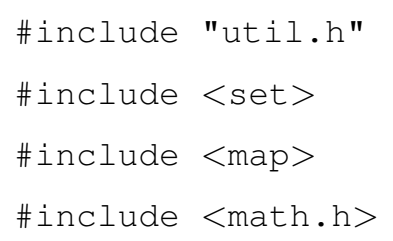

\section{Classes}

- class ibis::fileManager

This fileManager is intended to allow different objects to share the same open file. 
- class ibis::fileManager::cleaner

A function object to be used to register external cleaners.

- class ibis::fileManager::mutexLock

Used to prevent simultaneous modification of the two internal lists.

- class ibis::fileManager::readLock

An object who uses a file under the management of the file manager should hold a readLock.

- class ibis::fileManager::roFile

This class manages content of a whole (read-only) file.

- class ibis::fileManager::storage

The storage class treats all memory as char*.

- class ibis::fileManager::writeLock

A write lock for controlling access to the two interval lists.

\section{Defines}

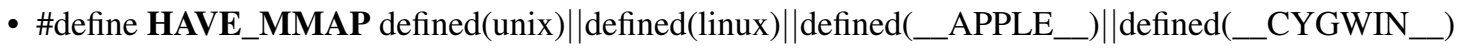

\subsubsection{Detailed Description}

Defines a simple file Manager.

\section{Note:}

Use malloc and realloc to manage memory when the file content is actually in memory. The main reason for doing so is to malloc for resizing. This may potentially cause problems with memory allocation through the new operator provided by $\mathrm{C}++$ compiler.

\subsection{2 horometer.h File Reference}

Defines a simple timer class.

\#include <stdio.h>

\#include <time.h>

\section{Namespaces}

- namespace ibis

\section{Classes}

- class ibis::horometer

Horometer - a primitive timing instrument.

\subsubsection{Detailed Description}

Defines a simple timer class. 


\subsection{3 ibin.h File Reference}

Define ibis::bin and derived classes.

\#include "index.h"

\section{Classes}

- class ibis::ambit

The multi-level range based (cumulative) index.

- class ibis::bak

Maps each value to a lower prevision (decimal) values and use the the low precision value as center of the bin.

- class ibis::bak2

A variation on ibis: : bak, it splits each bin of ibis::bak in two, one for entries less than the mapped value and one for the entries that greater and equal to the mapped value.

- struct ibis::bak2::grain

A simple structure to record the position of the values mapped to the same value.

- struct ibis::bak::grain

- class ibis::bin

The equality encoded bitmap index with binning.

- struct ibis::bin::granule

A data structure to assist the mapping of values to lower precisions.

- class ibis::egale

The multicomponent equality code on bins.

- class ibis::entre

The multicomponent interval code on bins.

- class ibis::mesa

This class implements the two-side range encoding from Chan and Ioannidis.

- class ibis::moins

The multicomponent range code on bins.

- class ibis::pack

A two-level index.

- class ibis::pale

A two-level index.

- class ibis::range

The range encoded bitmap index based.

- class ibis::zone

A two-level index. 


\subsubsection{Detailed Description}

Define ibis::bin and derived classes.

bin -> range, mesa, ambit, pale, pack, zone, egale, bak, bak2
egale -> moins, entre

\subsection{4 ibis.h File Reference}

The header file to be included by all user code.

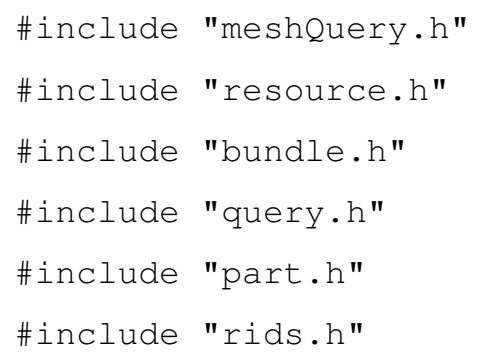

\section{Namespaces}

- namespace ibis

\section{Functions}

- void ibis::init (const int verbose $=0$, const char $*$ rcfile $=0$ )

Initializes internal resources required by ibis.

\subsubsection{Detailed Description}

The header file to be included by all user code.

It defines all classes and functions intended to use ibis: : part interface. All such classes and functions are defined in the namespace ibis. Before performing any operations, the first function to be called is ibis: : init.

\section{See also:}

ibis::init

\subsection{5 idirekte.h File Reference}

This is an implementation of the the simple bitmap index without the first binning step.

\#include "index.h"

\section{Classes}

- class ibis::direkte

Directly use the integer values as bin number to avoid some intemdiate steps. 


\subsubsection{Detailed Description}

This is an implementation of the the simple bitmap index without the first binning step.

It directly uses the integer values as bin number. The word direkte in Danish means direct.

\subsection{6 ikeywords.h File Reference}

This index is a keyword index for a string-valued column.

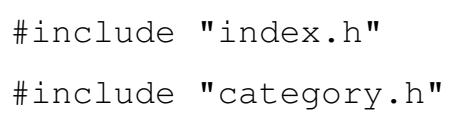

\section{Classes}

- class ibis::keywords

Class ibis: : keywords defines a boolean term-document matrix.

\subsubsection{Detailed Description}

This index is a keyword index for a string-valued column.

It contains a boolean version of the term-document matrix and supports exact matches of keywords/terms.

\subsection{7 index.h File Reference}

Definition of the common functions of an index.

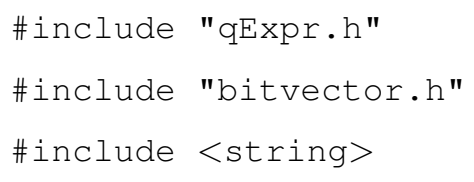

\section{Namespaces}

- namespace ibis

\section{Classes}

- class ibis::index

The base index class.

- class ibis::index::barrel

A specialization that adds function setValue.

\subsubsection{Detailed Description}

Definition of the common functions of an index.

The index class is a pure virtual base class with a static create function and a few virtual functions that provide common functionality. 
An index is built for each individual column (ibis::column) of a data table. The primary function of the index is to compute the solution or an estimation (as a pair of upper and lower bounds) for a range query. It needs to be generated and updated as necessary. The simplest way of generating an index is to build one from a file containing the binary values of a column. An index can only be updated for new records appended to the data table. Any other form of update, such as removal of some records, change some existing records can only be updated by removing the existing index then recreate the index.

\subsection{8 irelic.h File Reference}

Define ibis::relic and its derived classes.

\#include "index.h"

\section{Classes}

- class ibis::bylt

The two-level range-equality code.

- class ibis::fade

The multicomponent range-encoded index.

- class ibis::fuzz

The two-level interval-equality code.

- class ibis::relic

The basic bitmap index.

- class ibis::sapid

The multicomponent equality encoded index.

- class ibis::sbiad

The multicomponent interval encoded index.

- class ibis::slice

The bit-sliced index (O'Neil). It used the binary encoding.

- class ibis::zona

The two-level equality-equality code.

\subsubsection{Detailed Description}

Define ibis::relic and its derived classes.

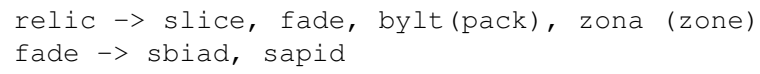

\subsection{9 iroster.h File Reference}

Defines a pseudo-index.

\#include "array_t.h"

\#include "util.h" 


\section{Classes}

- class ibis::roster

A roster list is a list of indices for ordering the values in the ascending order.

\subsubsection{Detailed Description}

Defines a pseudo-index.

Used in some performance comparisons.

\subsection{0 mensa.h File Reference}

A table with multiple data partitions on disk.

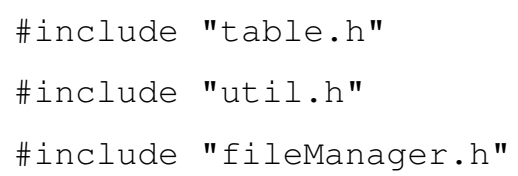

\section{Namespaces}

- namespace ibis

\section{Classes}

- class ibis::mensa

Class ibis::mensa contains multiple (horizontal) data partitions (ibis::part) to form a logical data table.

- class ibis::mensa::cursor

- struct ibis::mensa::cursor::bufferElement

\subsubsection{Detailed Description}

A table with multiple data partitions on disk.

This class defines the data structure to encapsulate multiple on-disk data partitions into a logical table. The class translates the function defined on ibis: : part to the ibis: : table interface.

\subsection{1 meshQuery.h File Reference}

The header file defining an extension of query on mesh data.

\#include "query.h"

\section{Namespaces}

- namespace ibis 


\section{Classes}

- class ibis::meshQuery

The class adds more functionality to ibis::query to handle data from meshes.

\subsubsection{Detailed Description}

The header file defining an extension of query on mesh data.

\subsection{2 part.h File Reference}

Define the class ibis::part.

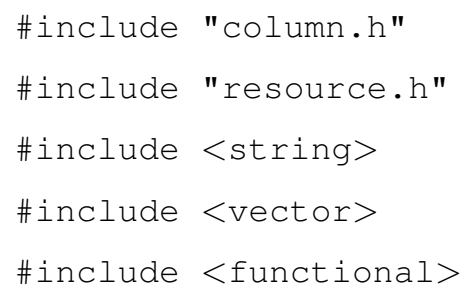

\section{Namespaces}

- namespace ibis

- namespace ibis::util

\section{Classes}

- class ibis::part

The class ibis::part represents a partition of a relational table.

- class ibis::part::advisoryLock

An non-blocking version of writeLock.

- class ibis::part::barrel

To read a list of variables at the same time.

- class ibis::part::cleaner

A cleaner to be used by the fileManager::unload function.

- struct ibis::part::indexBuilderPool

- struct ibis::part::info

A simple class to describe an ibis::part object.

- class ibis::part::mutexLock

Provide a mutual exclusion lock on an ibis::part object.

- class ibis::part::readLock

Provide a read lock on an ibis::part.

- struct ibis::part::thrArg

- class ibis::part::vault 
To read variables in certain order.

- class ibis::part::writeLock

Provide a write lock on an ibis::part.

\section{Functions}

- void ibis::util::tablesFromDir (ibis::partList \&tlist, const char $* \operatorname{dir} 1$ )

Look into the given directory for table.tdc files.

- void ibis::util::tablesFromDir (ibis::partList \&tables, const char $*$ adir, const char $*$ bdir)

Look for data directories in the given pair of directories.

- void ibis::util::tablesFromResources (ibis::partList \& tables, const ibis::resource \&res)

Reconstruct partitions using data directories specified in the resources.

\subsubsection{Detailed Description}

Define the class ibis::part.

This class defines some rudimentary functions for managing a vertically partitioned data partition and answering simple queries. It also provides limited number of functions to modify the data partition.

\subsection{3 predicate.h File Reference}

Replaces some default lex functions.

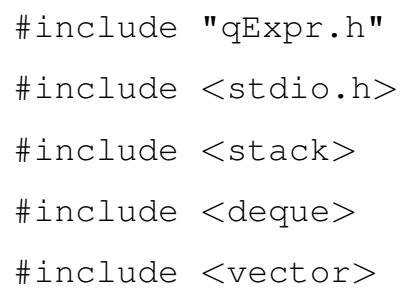

\section{Namespaces}

- namespace ibis

\section{Defines}

- \#define YYLMAX BUFSIZ

\section{Functions}

- int lex_input ()

- qExpr * ibis::parseQuery (const char $*$ str)

Parse a query string.

- void unput (int)

- void yyerror (const char $* \mathrm{~s}$ ) 
- int yylex ()

- int yyparse ()

- void yyparse_cleanup ()

- int yywrap ()

\section{Variables}

- int parse_length

- int parse_offset

- std::vector < char $*$ > parse_str_vec

- char $*$ parse_string

- char yytext [YYLMAX]

\subsubsection{Detailed Description}

Replaces some default lex functions.

The replacement functions all it to work on a string named parse_string (size parse_length). ALL lex/yacc related variables are in global namespace, only parseQuery is in the usual ibis namespace.

\section{Note:}

The only function that should be used elsewhere is parseQuery

ibis: : qExpr* ibis: parseQuery (const char* str)

\subsection{4 qExpr.h File Reference}

Define the query expression.

\#include "util.h"

\#include <functional>

\section{Namespaces}

- namespace ibis

\section{Classes}

- class ibis::compRange

The class compRange stores computed ranges.

- class ibis::compRange::barrel

A barrel to hold a list of variables.

- class ibis::compRange::bediener

- class ibis::compRange::literal

- class ibis::compRange::number

- class ibis::compRange::stdFunction1

- class ibis::compRange::stdFunction2

- class ibis::compRange::term

- class ibis::compRange::variable

- class ibis::qAnyAny 
A user specifies this type of query expression with the following syntax,.

- class ibis::qContinuousRange

Simple range condition.

- class ibis::qDiscreteRange

- class ibis::qExpr

The top level query expression object.

- struct ibis::qExpr::weight

A functor to be used by the function reorder.

- class ibis::qMultiString

- class ibis::qRange

A class to represent simple range conditions.

- class ibis::qString

The class qString encapsulates information for comparing string values.

- class ibis::rangeJoin

A join is defined by two names and a numerical expression.

\section{Functions}

- std::ostream \& operator $<<$ (std::ostream \&out, const ibis::qExpr \&pn)

Wrap function print as operator $<<$.

\subsubsection{Detailed Description}

Define the query expression.

\subsection{5 query.h File Reference}

The header file defining the individual query objects.

\#include "part.h"

\#include $<$ map $>$

\section{Namespaces}

- namespace ibis

\section{Classes}

- class ibis::query

A data structure for representing user queries.

- class ibis::query::readLock

- class ibis::query::weight

- class ibis::query::writeLock 


\subsubsection{Detailed Description}

The header file defining the individual query objects.

\subsection{6 resource.h File Reference}

Defines a class to hold name-value pairs.

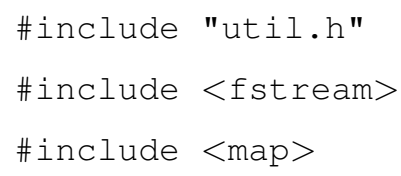

\section{Namespaces}

- namespace ibis

\section{Classes}

- class ibis::resource

A container for name-value pairs.

\section{Functions}

- ibis::resource \& ibis::gParameters ()

The reference to the global configuration parameters.

\subsubsection{Detailed Description}

Defines a class to hold name-value pairs.

\subsection{7 rids.h File Reference}

Define simple IO functions for ibis::rid_t.

\#include "util.h"

\section{Namespaces}

- namespace ibis

\section{Classes}

- class ibis::ridHandler

A class for handling file IO for ibis::rid_t. 


\subsubsection{Detailed Description}

Define simple IO functions for ibis::rid_t.

Based on on OidIOHandler by David Malon <malon@anl.gov>.

\subsection{8 tab.h File Reference}

This file stores two trivial concrete classes of ibis::table: tabula and tabele.

\#include "table.h"
\#include <iostream>

Namespaces

- namespace ibis

\section{Classes}

- class ibis::tabele

A trivial class for table with one row and one column.

- class ibis::tabele::cursor

- class ibis::tabula

A trivial class for table with no columns.

- class ibis::tabula::cursor

\subsubsection{Detailed Description}

This file stores two trivial concrete classes of ibis::table: tabula and tabele.

Here is an explanation of how these two words are related to "table".

\section{Remarks:}

The term "table" is derived from a merger of French table and Old English tabele, ultimately from the Latin word tabula, "a board, plank, flat piece". In Late Latin, tabula took over the meaning previously reserved to mensa (preserved in Spanish mesa "table"). In Old English, the word replaced bord for this meaning. - Wikipedia.

\subsection{9 table.h File Reference}

FastBit Table Interface.

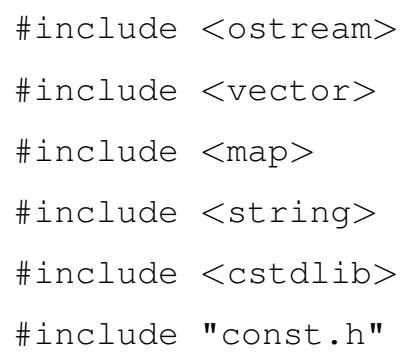




\section{Namespaces}

- namespace ibis

\section{Classes}

- class ibis::table

The abstract table class.

- class ibis::table::cursor

Cursor class for row-wise data accesses.

- struct ibis::table::row

A simple struct for storing a row of a table.

- class ibis::tableList

A list of tables.

- class ibis::tablex

The class for expandable tables.

\section{Enumerations}

- enum ibis::TYPE_T \{

ibis::UNKNOWN_TYPE = 0, ibis::OID, ibis::BYTE, ibis::UBYTE, ibis::SHORT, ibis::USHORT, ibis::INT, ibis::UINT,

ibis::LONG, ibis::ULONG, ibis::FLOAT, ibis::DOUBLE, ibis::CATEGORY, ibis::TEXT \}

Supported data types.

\section{Variables}

- const char $*$ ibis::TYPECODE

One-character code for the enumeration types.

- const char $* *$ ibis::TYPESTRING

Human readable version of the enumeration types.

\subsubsection{Detailed Description}

FastBit Table Interface.

This is intended to be a facade for FastBit functions that provide a view that is all operations are on tables. Two main classes are defined here, table and tablex. The class table is intended to be used with read-only data and it provides mostly querying functions. The class tablex is intended for users to add new records to the existing records. 


\subsection{0 tafel.h File Reference}

An expandable table.

\#include "table.h"

\#include "bitvector.h"

\section{Namespaces}

- namespace ibis

\section{Classes}

- class ibis::tafel

An expandable table.

- struct ibis::tafel::column

\subsubsection{Detailed Description}

An expandable table.

This file defines ibis: : tafel.

\subsection{1 twister.h File Reference}

Pseudorandom number generators.

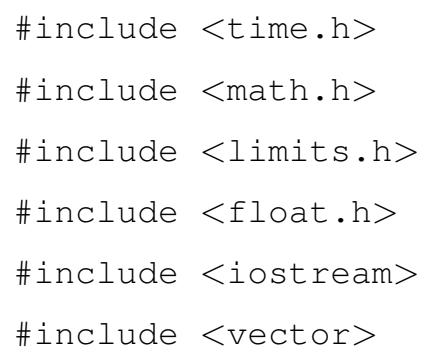

\section{Namespaces}

- namespace ibis

\section{Classes}

- class ibis::discretePoisson

Discrete random number with Poisson distribution $\exp (-x /$ lambda).

- class ibis::discretePoisson1 Specialized version of the Poisson distribution $\exp (-x)$.

- class ibis::discreteZipf

Discrete Zipf distribution: $p(k)$ is proportional to $(v+k)^{\wedge}(-a)$ where $a>1, k>=0$.

- class ibis::discreteZipf1 
A specialized case of the Zipf distribution $f(x)=1 /(1+x)$.

- class ibis::discreteZipf2

A specialized version of the Zipf distribution $f(x)=1 /(1+x)^{\wedge} 2$.

- class ibis::MersenneTwister

Mersenne Twister generates uniform random numbers efficiently.

- class ibis::uniformRandomNumber

A functor to generate uniform random number in the range $[0,1)$.

\subsubsection{Detailed Description}

Pseudorandom number generators.

MersenneTwister: A C++ class that use the similar interface as java.util.Random. The basic algorithm is based on the Mersenne Twister by M. Matsumoto and T. Nishimura.

MersenneTwister also include a function called nextZipf to generate Zipf distributed random numbers (floats).

This file also contains additional classes that generate discrete Zipf and Poisson distributions (named discreteZipf and discretePoisson)

\subsection{2 util.h File Reference}

Defines minor utility functions and common classes used by FastBit.

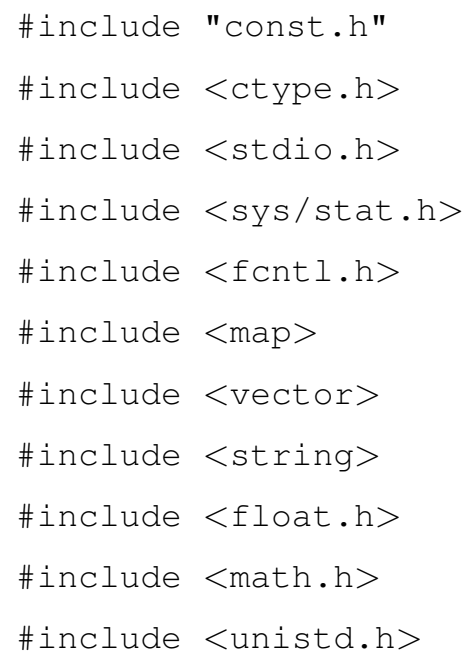

\section{Namespaces}

- namespace ibis

- namespace ibis::util

- namespace std

\section{Classes}

- class ibis::bad_alloc

A specialization of std::bad_alloc. 
- class ibis::nameList

A data structure to store a small set of names.

- class ibis::selected

A data structure to store the select clause of a query.

- class ibis::util::counter

A simple global counter.

- class ibis::util::ioLock

A global IO mutex lock.

- class ibis::util::mutexLock

An wrapper class for perform pthread_mutex_lock/unlock.

- class ibis::util::quietLock

An wrapper class for perform pthread_mutex_lock/unlock.

- class ibis::util::readLock

An wrapper class for perform pthread_rwlock_rdlock/unlock.

- class ibis::util::writeLock

An wrapper class for perform pthread_rwlock_wrlock/unlock.

- struct std::less $<$ char $*>$

- struct std::less $<$ const char $*>$

- struct std::less $<$ const ibis::rid_t $*>$

- struct std::less< $<$ ibis::rid_t $>$

\section{Defines}

- \#define DBL_EPSILON 2.2204460492503131e-16

- \#define OPEN_FILEMODE S_IRUSR | S_IWUSR | S_IRGRP | S_IWGRP | S_IROTH

- \#define Stat_T struct stat

- \#define UnixClose ::close

- \#define UnixFStat ::fstat

- \#define UnixOpen ::open

- \#define UnixRead ::read

- \#define UnixSeek ::lseek

- \#define UnixSnprintf ::snprintf

- \#define UnixStat ::stat

- \#define UnixWrite ::write

\section{Typedefs}

- typedef std::vector $<$ colValues $*>$ ibis::colList

- typedef std::map $<$ const char $*$, part $*$, lessi $>$ ibis::partList

- typedef array_t $<$ rid_t $>$ ibis::RIDSet 


\section{Functions}

- uint32_t ibis::util::checksum (uint32_t a, uint32_t b)

Fletcher's checksum on two integers. Returns an integer.

- double ibis::util::coarsen (const double in, const unsigned prec)

Reduce the decimal precision of the incoming floating-point value to specified precision.

- int ibis::util::copy (const char $*$ to, const char $*$ from)

Copy "from" to "to".

- uint32_t ibis::util::getBuffer (char $* \&$ buf)

Generate a reasonably sized buffer for storing temporary contents.

- off_t ibis::util:::getFileSize (const char $*$ name)

Return size of the file in bytes.

- void ibis::util::getGMTime (char $*$ str)

- void ibis::util:::getLocalTime (char $*$ str)

Return the current time in string format as asct ime_r.

- void ibis::util:: getString (std::string \&str, const char $* \&$ buf, const char $*$ delim)

Extract the next quoted string or the blank delimited string.

- char $*$ ibis::util:::getString (const char $*$ buf)

Treat all bytes in buf as the string.

- const char $*$ ibis::util::getToken (char $* \&$ str, const char $*$ tok_chrs)

- char $*$ ibis::util::itoa (int value, char $*$ str, int)

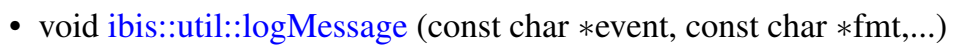

Print a message to standard output.

- int ibis::util::makeDir (const char $*$ dir)

Recursively create the name directory.

- std::ostream \& operator $<<$ (std::ostream \&out, const ibis::rid_t \&rid)

Print a rid_t to an output stream.

- std::istream \& operator $>>$ (std::istream \&is, ibis::rid_t \&rid)

Read a rid_t from an input stream.

- const ibis::bitvector64 \& ibis::outerProduct (const ibis::bitvector \&a, const ibis::bitvector \&b, ibis::bitvector64 \&c)

Compute the outer product of a and $\mathrm{b}$, add the result to $\mathrm{c}$.

- const ibis::bitvector64 \& ibis::outerProductUpper (const ibis::bitvector \&a, const ibis::bitvector \&b, ibis::bitvector64 \&c)

Add the strict upper triangular portion of the outer production between a and $\mathrm{b}$ to $\mathrm{c}$.

- double ibis::util::rand ()

A pseudo-random number generator $(0,1)$.

- double ibis::util::rand () 
A pseudo-random number generator $(0,1)$.

- int ibis::util:::readDouble (double \&val, const char $* \&$ str, const char $*$ del)

Attempt to convert the incoming string into a double.

- int ibis::util::readInt (int64_t \&val, const char $* \& s t r$, const char $*$ del)

Attempt to convert the incoming string into an integer.

- void ibis::util::removeDir (const char $*$ name, bool leaveDir=false)

Remove the content of named directory.

- void ibis::util::removeTail (char $*$ str, char tail)

Remove trailing character 'tail' from str.

- void ibis::util::removeTail (char $*$ str, char tail)

Remove trailing character 'tail' from str.

- void ibis::util::secondsToString (const time_t, char $* s t r$ )

- std::string ibis::util::shortName (const std::string \&de)

- bool ibis::util::strMatch (const char $*$ str, const char $*$ pat)

Match the string.

- char $*$ ibis::util::strnewdup (const char $*$ s, const uint32_t $\mathrm{n}$ )

- char $*$ ibis::util::strnewdup (const char $* \mathrm{~s}$ )

duplicate string content with $C++$ default new operator

- char $*$ ibis: :util::strnewdup (const char $*$ s, const uint32_t n)

- char $*$ ibis::util::strnewdup (const char $*$ s)

duplicate string content with $\mathrm{C}++$ default new operator

- char $*$ ibis::util::trim (char $*$ str)

Remove leading and trailing blank space.

- char $*$ ibis::util::trim (char $*$ str)

Remove leading and trailing blank space.

- void ibis::util::uniformFraction (const long unsigned idx, long unsigned \&denominator, long unsigned \&numerator)

Compute a denominator and numerator pair to compute a uniform distribution of numbers in a given range.

- unsigned long ibis::util::uniqueNumber ()

Return an integer that is always increasing.

- const char $*$ ibis::util::userName ()

Return the name of the user who is running the program.

- uint32_t ibis::util::checksum (uint32_t a, uint32_t b)

Fletcher's checksum on two integers. Returns an integer.

- uint32_t ibis::util::checksum (const char $*$ str, uint32_t sz)

Fletcher's arithmetic checksum with 32-bit result.

- std::string ibis::util::shortName (const std::string \&longname) 
Fletcher's checksum on two integers. Returns an integer.

- double ibis::util::coarsen (const double in, const unsigned prec=2)

Reduce the decimal precision of the incoming floating-point value to specified precision.

- double ibis::util::compactValue (double left, double right, double start=0.0)

Compute a compact 64-bit floating-point value with a short decimal representation in the range (left, right].

- double ibis::util::decrDouble (const double \&in)

Reduce the decimal precision of the incoming floating-point value to specified precision.

- void ibis::util::eq2range (const double \&in, double \&left, double \&right)

Reduce the decimal precision of the incoming floating-point value to specified precision.

- double ibis::util::incrDouble (const double \&in)

Functions to handle manipulation of floating-point numbers.

- void ibis::util::setNaN (float \&val)

Reduce the decimal precision of the incoming floating-point value to specified precision.

- void ibis::util::setNaN (double \&val)

Set a double to NaN.

- void ibis::util::int2string (std::string \&str, const std::vector < unsigned $>\&$ val)

- void ibis::util::int2string (std::string \&str, unsigned v1, unsigned v2, unsigned v3)

- void ibis::util::int2string (std::string \&str, unsigned v1, unsigned v2)

- void ibis::utill::int2string (std::string \&str, unsigned val)

convert 32-bit integers to base-64 printable characters

- void ibis::util::isortRIDs (ibis::RIDSet \&, uint32_t, uint32_t)

- void ibis::util::sortRIDs (ibis::RIDSet \&, uint32_t, uint32_t)

- void ibis::util::sortRIDs (ibis::RIDSet \&rids)

Sorting RID lists.

\section{Variables}

- const short unsigned ibis::util::charIndex []

- const char $*$ ibis::util::charTable

charTable lists the 64 printable characters to be used for names charIndex maps the characters (ASCII) back to integer [0-64]

-pthread_mutex_t ibis::util::envLock

A mutex intended to be used for ensuring there is only one function that modifies the environment and other conditions.

\subsubsection{Detailed Description}

Defines minor utility functions and common classes used by FastBit. 


\section{Index}

ACCESS PREFERENCE

ibis::fileManager, 112

actualMinMax

ibis::column, 85

add

ibis::resource, 206

ibis::tableList, 236

addBins

addBits

ibis::index, 125

ibis::index, 125

adjustSize

ibis::bitvector, 40

ibis::bitvector64, 45

append

ibis::column, 85

ibis::part, 165

ibis::relic, 202

ibis::ridHandler, 209

ibis::tablex, 238

ibis::tafel, 248

ibis::text, 250

appendRow

ibis::tablex, 238

ibis::tafel, 248

appendRows

ibis::tablex, 239

ibis::tafel, 248

appendToBackup

ibis::part, 165

array_t, 14

array_t, 17

bottomk, 17

deepCopy, 17

find, 18

insert, 18

nosharing, 18

operator[], 18

reserve, 18

size, 18

stableSort, 18, 19

topk, 19

array_t.h, 259

binning

ibis::bin, 32

bitvector

ibis::bitvector, 40

bitvector.h, 259

bitvector64.h, 260

bitvectorToCoordinates ibis::meshQuery, 145

bord.h, 261

bottomk array_t, 17

buildIndex

ibis::bord, 53

ibis::mensa, 138

ibis::tabele, 220

ibis::table, 228

ibis::tabula, 242

buildIndexes

ibis::bord, 53

ibis::mensa, 138

ibis::tabele, 221

ibis::table, 228

ibis::tabula, 242

bundle.h, 261

bylt

ibis::bylt, 66

BYTE

ibis, 9

capi.h, 262

fastbit_build_query, 265

fastbit_destroy_query, 265

fastbit_get_qualified_int, 265

fastbit_get_result_rows, 265

fastbit_init, 265

fastbit_result_set_getInt, 265

CATEGORY

ibis, 9

category.h, 265

checkBin

ibis::bin, 32

clusteringFactor

ibis::bitvector, 40

ibis::bitvector64, 45

column

ibis::column, 85

column.h, 266

colValues.h, 267

commit

ibis::part, 165

computeMinMax

ibis::column, 85, 86

const.h, 267

PREFERRED_BLOCK_SIZE, 269

THREAD_RWLOCK_INITIALIZER, 269

construct

ibis::relic, 202

contractQuery

ibis::query, 189

countDeltaPairs

ibis::query, 189

countEqualPairs

ibis::query, 189

countHits 
ibis::query, 190

create

ibis::index, 125

ibis::table, 228

dataFileName

ibis::column, 86

deepCopy

array_t, 17

divideCounts

ibis::index, 126

doCompare

ibis::part, 165, 166

doScan

ibis::part, 166

DOUBLE

ibis, 9

dump

ibis::bord, 53

ibis::mensa, 138

ibis::tabele, 221

ibis::table, 228

ibis::tabula, 242

end

ibis::tableList, 236

estimate

ibis::ambit, 21

ibis::bin, 32, 33

ibis::bord, 53

ibis::direkte, 98

ibis::egale, 104

ibis::entre, 107

ibis::index, 126, 127

ibis::keywords, 133

ibis::mensa, 138

ibis::mesa, 143

ibis::moins, 148

ibis::pack, 151

ibis::pale, 154

ibis::query, 190

ibis::range, 197

ibis::relic, 202, 203

ibis::slice, 217

ibis::tabele, 221

ibis::table, 228

ibis::tabula, 242

ibis::zone, 257

estimateMatchAny

ibis::part, 166

estimateRange

ibis::column, 86

evaluate

ibis::ambit, 21

ibis::bin, 33

ibis::bylt, 66

ibis::direkte, 99 ibis::egale, 104

ibis::entre, 107

ibis::fade, 109

ibis::fuzz, 118

ibis::index, 127

ibis::keywords, 133

ibis::mesa, 143

ibis::moins, 148

ibis::pack, 151

ibis::pale, 154

ibis::query, 190

ibis::range, 197

ibis::relic, 203

ibis::sapid, 212

ibis::sbiad, 213

ibis::slice, 217

ibis::zona, 255

ibis::zone, 258

evaluateJoin

ibis::part, 166

evaluateRange

ibis::column, 86

expandQuery

ibis::query, 190

expandRange

ibis::bak, 24

ibis::bak2, 26

ibis::bin, 33

ibis::index, 127

ibis::range, 197

fastbit_build_query

capi.h, 265

fastbit_destroy_query

capi.h, 265

fastbit_get_qualified_int

capi.h, 265

fastbit_get_result_rows

capi.h, 265

fastbit_init

capi.h, 265

fastbit_result_set_getInt

capi.h, 265

fetch

ibis::table::cursor, 233

fileManager.h, 269

find

array_t, 18

ibis::dictionary, 96

ibis::nameList, 149

ibis::selected, 215

findString

ibis::column, 86

ibis::text, 250

flip

ibis::bitvector, 40

ibis::bitvector64, 45 
FLOAT

ibis, 9

fuzz

ibis::fuzz, 118

get1DDistribution

ibis::part, 167

getActualMax

ibis::column, 86

getActualMin

ibis::column, 86

getColumn

ibis::part, 167

getColumnAsByte

ibis::table::cursor, 234

getColumnAsBytes

ibis::bord, 53

ibis::mensa, 138

ibis::tabele, 221

ibis::table, 228

ibis::tabula, 242

getColumnAsDoubles

ibis::bord, 53

ibis::mensa, 138

ibis::tabele, 221

ibis::table, 229

ibis::tabula, 243

getColumnAsFloats

ibis::bord, 53

ibis::tabele, 221

ibis::table, 229

ibis::tabula, 243

getColumnAsInts

ibis::bord, 54

ibis::mensa, 138

ibis::tabele, 221

ibis::table, 229

ibis::tabula, 243

getColumnAsLongs

ibis::bord, 54

ibis::mensa, 139

ibis::tabele, 222

ibis::table, 229

ibis::tabula, 243

getColumnAsShorts

ibis::bord, 54

ibis::mensa, 139

ibis::tabele, 222

ibis::table, 229

ibis::tabula, 243

getColumnAsStrings

ibis::bord, 54

ibis::tabele, 222

ibis::table, 230

ibis::tabula, 243

getColumnAsUBytes

ibis::bord, 54 ibis::mensa, 139

ibis::tabele, 222

ibis::table, 230

ibis::tabula, 244

getColumnAsUInts

ibis::bord, 54

ibis::mensa, 139

ibis::tabele, 222

ibis::table, 230

ibis::tabula, 244

getColumnAsULongs

ibis::bord, 55

ibis::tabele, 222

ibis::table, 230

ibis::tabula, 244

getColumnAsUShorts

ibis::bord, 55

ibis::mensa, 139

ibis::tabele, 223

ibis::table, 230

ibis::tabula, 244

getCumulativeDistribution ibis::column, 86

ibis::part, 167

getCurrentRowNumber

ibis::table::cursor, 234

getDistribution

ibis::column, 87

ibis::part, 168

getFile

ibis::fileManager, 112

getFileSegment

ibis::fileManager, 112

getGroup

ibis:::resource, 206

getHistogram

ibis::bord, 55

ibis::mensa, 139

ibis::tabele, 223

ibis::table, 230

ibis::tabula, 244

getHistogram2D

ibis::bord, 55

ibis::mensa, 140

ibis::tabele, 223

ibis::table, 231

ibis::tabula, 245

getHistogram3D

ibis::bord, 55

ibis::mensa, 140

ibis::tabele, 223

ibis::table, 231

ibis::tabula, 245

getHitsAsRanges

ibis::meshQuery, 145

getHitVector

ibis::query, 190 


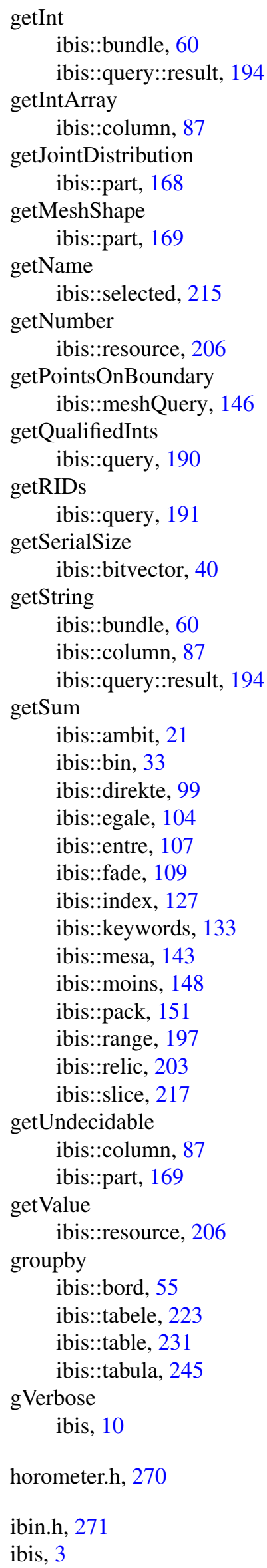

BYTE, 9

CATEGORY, 9

DOUBLE, 9

FLOAT, 9

gVerbose, 10

init, 9

INT, 9

LONG, 9

OID, 9

outerProduct, 9

parseQuery, 10

SHORT, 9

TEXT, 9

TYPE_T, 9

UBYTE, 9

UINT, 9

ULONG, 9

UNKNOWN_TYPE, 9

USHORT, 9

ibis.h, 272

ibis::ambit, 19

estimate, 21

evaluate, 21

getSum, 21

print, 21

read, 21

undecidable, 21

write, 22

ibis::bad_alloc, 22

ibis::bak, 23

expandRange, 24

print, 24

read, 24

write, 24

ibis::bak2, 25

expandRange, 26

print, 26

read, 26

write, 26

ibis::bak2::grain, 27

ibis::bin, 27

binning, 32

checkBin, 32

estimate, 32,33

evaluate, 33

expandRange, 33

getSum, 33

print, 33

read, 34

setBoundaries, 34

undecidable, 34

write, 35

ibis::bin::granule, 35

ibis::bitvector, 36

adjustSize, 40

bitvector, 40

clusteringFactor, 40 
flip, 40

getSerialSize, 40

markovSize, 40

operator $=, 40$

randomSize, 40

read, 40

set, 41

setBit, 41

setSize, 41

write, 41

ibis::bitvector64, 41

adjustSize, 45

clusteringFactor, 45

flip, 45

markovSize, 45

randomSize, 45

read, 45

set, 46

setBit, 46

ibis::bitvector64::const_iterator, 46

ibis::bitvector64::indexSet, 47

ibis::bitvector64::iterator, 47

ibis::bitvector::const_iterator, 48

ibis::bitvector::indexSet, 48

ibis::bitvector::iterator, 49

ibis::bord, 50

buildIndex, 53

buildIndexes, 53

dump, 53

estimate, 53

getColumnAsBytes, 53

getColumnAsDoubles, 53

getColumnAsFloats, 53

getColumnAsInts, 54

getColumnAsLongs, 54

getColumnAsShorts, 54

getColumnAsStrings, 54

getColumnAsUBytes, 54

getColumnAsUInts, 54

getColumnAsULongs, 55

getColumnAsUShorts, 55

getHistogram, 55

getHistogram2D, 55

getHistogram3D, 55

groupby, 55

indexSpec, 56

orderby, 56

ibis::bord::column, 56

selectDoubles, 57

selectLongs, 57

ibis::bundle, 58

getInt, 60

getString, 60

rowCounts, 60

ibis::bundle 0,61

ibis::bundle1, 61

ibis::bundles, 63 reorder, 64

truncate, 64

ibis::bylt, 65

bylt, 66

evaluate, 66

print, 66

read, 66, 67

write, 67

ibis::category, 67

ibis::colDoubles, 68

ibis::colDoubles

reorder, 70

sort, 70

ibis::colFloats, 70

ibis::colFloats

reorder, 72

sort, 72

ibis::colInts, 72

ibis::colInts

reorder, 74

sort, 74

ibis::colLongs, 75

ibis::colLongs

reorder, 76

sort, 76

ibis::colUInts, 77

ibis::colUInts

reorder, 78

sort, 78

ibis::colULongs, 79

ibis::colULongs

reorder, 80

sort, 80

ibis::column, 81

actualMinMax, 85

append, 85

column, 85

computeMinMax, 85, 86

dataFileName, 86

estimateRange, 86

evaluateRange, 86

findString, 86

getActualMax, 86

getActualMin, 86

getCumulativeDistribution, 86

getDistribution, 87

getIntArray, 87

getString, 87

getUndecidable, 87

selectBytes, 87

selectDoubles, 87

selectLongs, 87

selectShorts, 87

selectValues, 88

truncateData, 88

ibis:: column::indexLock, 88

ibis::column::info, 88 
ibis::column::mutexLock, 89

ibis::column::writeLock, 89

ibis::colValues, 90

ibis::colValues

reorder, 92

sort, 92

ibis::compRange, 92

ibis::compRange::barrel, 94

ibis::compRange::barrel recordVariable, 95

ibis::dictionary, 95

find, 96

insertRaw, 96

ibis::direkte, 96

estimate, 98

evaluate, 99

getSum, 99

print, 99

read, 99

undecidable, 99

ibis::discretePoisson, 100

ibis::discretePoisson 1, 100

ibis::discreteZipf, 101

ibis::discreteZipf 1, 101

ibis::discreteZipf2, 101

ibis::egale, 102

estimate, 104

evaluate, 104

getSum, 104

print, 104

read, 104

undecidable, 105

write, 105

ibis::entre, 105

estimate, 107

evaluate, 107

getSum, 107

print, 107

write, 107

ibis::fade, 107

evaluate, 109

getSum, 109

print, 109

write, 109

ibis::fileManager, 110

ibis::fileManager

ACCESS_PREFERENCE, 112

getFile, 112

getFileSegment, 112

recordPages, 112

tryGetFile, 113

ibis::fileManager::cleaner, 113

ibis::fileManager::readLock, 113

ibis::fileManager::roFile, 114

ibis::fileManager::roFile

score, 114

ibis::fileManager::storage, 115
ibis::fileManager::storage

inUse, 116

pastUse, 116

unnamed, 116

ibis::fuzz, 116

evaluate, 118

fuzz, 118

print, 118

read, 118

write, 118

ibis::horometer, 119

resume, 119

start, 119

stop, 119

ibis::index, 120

addBins, 125

addBits, 125

create, 125

divideCounts, 126

estimate, 126, 127

evaluate, 127

expandRange, 127

getSum, 127

index, 125

indexFileName, 127

isIndex, 127

mapValues, 128

print, 128

read, 129

setBases, 129

str, 130

sumBins, 129

sumBits, 129, 130

undecidable, 130

write, 130

ibis::index::barrel, 130

ibis::keywords, 131

estimate, 133

evaluate, 133

getSum, 133

keywords, 133

print, 133

read, 134

readKeyword, 134

readTermDocFile, 134

ibis::lessi, 134

ibis::mensa, 135

buildIndex, 138

buildIndexes, 138

dump, 138

estimate, 138

getColumnAsBytes, 138

getColumnAsDoubles, 138

getColumnAsInts, 138

getColumnAsLongs, 139

getColumnAsShorts, 139

getColumnAsUBytes, 139 
getColumnAsUInts, 139

getColumnAsUShorts, 139

getHistogram, 139

getHistogram2D, 140

getHistogram3D, 140

indexSpec, 140

orderby, 140

ibis::MersenneTwister, 141

ibis::mesa, 141

estimate, 143

evaluate, 143

getSum, 143

print, 143

undecidable, 143

write, 144

ibis::meshQuery, 144

ibis::meshQuery

bitvectorToCoordinates, 145

getHitsAsRanges, 145

getPointsOnBoundary, 146

ibis::moins, 146

estimate, 148

evaluate, 148

getSum, 148

print, 148

write, 148

ibis::nameList, 149

ibis::nameList

find, 149

ibis::pack, 150

estimate, 151

evaluate, 151

getSum, 151

print, 151

read, 152

undecidable, 152

write, 152

ibis::pale, 152

estimate, 154

evaluate, 154

print, 154

read, 154

undecidable, 154

write, 155

ibis::part, 155

append, 165

appendToBackup, 165

commit, 165

doCompare, 165, 166

doScan, 166

estimateMatchAny, 166

evaluateJoin, 166

get1DDistribution, 167

getColumn, 167

getCumulativeDistribution, 167

getDistribution, 168

getJointDistribution, 168
getMeshShape, 169

getUndecidable, 169

loadIndex, 169

lookforString, 169

matchAny, 169

metaTags, 169

part, 165

purgeIndexFiles, 169

readTDC, 169

reorder, 170

rollback, 170

setMeshShape, 170

setMetaTags, 170

ibis::part::advisoryLock, 170

ibis::part::barrel, 171

read, 172

seek, 172

ibis::part::cleaner, 172

ibis::part::info, 172

ibis::part::mutexLock, 173

ibis::part::readLock, 173

ibis::part::vault, 174

read, 175

tellReal, 175

ibis::part::writeLock, 175

ibis::qAnyAny, 175

ibis::qAnyAny printRange, 176

ibis::qContinuousRange, 176

ibis::qContinuousRange

inRange, 178

printRange, 178

ibis::qExpr, 178

ibis::qExpr

printRange, 180

separateSimple, 180

simplify, 180

TYPE, 180

ibis::qExpr::weight, 181

ibis::qRange, 181

ibis::qRange

inRange, 182

ibis::qString, 182

ibis::query, 183

contractQuery, 189

countDeltaPairs, 189

countEqualPairs, 189

countHits, 190

estimate, 190

evaluate, 190

expandQuery, 190

getHitVector, 190

getQualifiedInts, 190

getRIDs, 191

isValidToken, 191

limit, 191

logMessage, 191 
orderby, 191

orderPairs, 191

printSelected, 192

printSelectedWithRID, 192

processJoin, 192

query, 189

removeComplexConditions, 192

sequentialScan, 192

setSelectClause, 192

setTable, 192

setWhereClause, 193

sortEquiJoin, 193

sortRangeJoin, 193

ibis::query::result, 193

getInt, 194

getString, 194

reset, 195

ibis::range, 195

estimate, 197

evaluate, 197

expandRange, 197

getSum, 197

print, 197

read, 198

undecidable, 198

write, 198

ibis::rangeJoin, 198

ibis::relic, 199

append, 202

construct, 202

estimate, 202, 203

evaluate, 203

getSum, 203

print, 203

read, 203

relic, 202

write, 204

ibis::resource, 204

add, 206

getGroup, 206

getNumber, 206

getValue, 206

operator[], 206

parseNameValuePairs, 206

read, 206

write, 207

ibis::rid_t, 207

ibis::rid_t::name, 208

ibis:::ridHandler, 208

ibis::ridHandler

append, 209

read, 209

write, 209

ibis::roster, 209

locate, 211

mergeBlock2, 211

roster, 210
writeSorted, 211

ibis::sapid, 211

evaluate, 212

print, 212

write, 212

ibis::sbiad, 212

evaluate, 213

print, 214

write, 214

ibis::selected, 214

find, 215

getName, 215

ibis::slice, 215

estimate, 217

evaluate, 217

getSum, 217

print, 217

read, 217, 218

write, 218

ibis::tabele, 218

buildIndex, 220

buildIndexes, 221

dump, 221

estimate, 221

getColumnAsBytes, 221

getColumnAsDoubles, 221

getColumnAsFloats, 221

getColumnAsInts, 221

getColumnAsLongs, 222

getColumnAsShorts, 222

getColumnAsStrings, 222

getColumnAsUBytes, 222

getColumnAsUInts, 222

getColumnAsULongs, 222

getColumnAsUShorts, 223

getHistogram, 223

getHistogram2D, 223

getHistogram3D, 223

groupby, 223

indexSpec, 224

orderby, 224

ibis::table, 224

buildIndex, 228

buildIndexes, 228

create, 228

dump, 228

estimate, 228

getColumnAsBytes, 228

getColumnAsDoubles, 229

getColumnAsFloats, 229

getColumnAsInts, 229

getColumnAsLongs, 229

getColumnAsShorts, 229

getColumnAsStrings, 230

getColumnAsUBytes, 230

getColumnAsUInts, 230

getColumnAsULongs, 230 
getColumnAsUShorts, 230

getHistogram, 230

getHistogram2D, 231

getHistogram3D, 231

groupby, 231

indexSpec, 231

orderby, 232

parseNames, 232

ibis::table::cursor, 232

fetch, 233

getColumnAsByte, 234

getCurrentRowNumber, 234

ibis::table::row, 234

ibis::tableList, 235

ibis::tableList

add, 236

end, 236

operator[], 237

remove, 237

ibis::tablex, 237

append, 238

appendRow, 238

appendRows, 239

readCSV, 239

write, 239

ibis::tabula, 240

buildIndex, 242

buildIndexes, 242

dump, 242

estimate, 242

getColumnAsBytes, 242

getColumnAsDoubles, 243

getColumnAsFloats, 243

getColumnAsInts, 243

getColumnAsLongs, 243

getColumnAsShorts, 243

getColumnAsStrings, 243

getColumnAsUBytes, 244

getColumnAsUInts, 244

getColumnAsULongs, 244

getColumnAsUShorts, 244

getHistogram, 244

getHistogram2D, 245

getHistogram3D, 245

groupby, 245

indexSpec, 245

orderby, 245

ibis::tafel, 246

append, 248

appendRow, 248

appendRows, 248

readCSV, 248

write, 249

ibis::text, 249

append, 250

findString, 250

readString, 251
selectUInts, 251

startPositions, 251

ibis::uniformRandomNumber, 251

ibis::util::counter, 252

ibis::util::ioLock, 252

ibis::util::mutexLock, 253

ibis::util::quietLock, 253

ibis::util::readLock, 253

ibis::util::writeLock, 254

ibis::zona, 254

evaluate, 255

print, 255

read, 256

write, 256

zona, 255

ibis::zone, 256

estimate, 257

evaluate, 258

print, 258

read, 258

undecidable, 258

write, 258

idirekte.h, 272

ikeywords.h, 273

index

ibis::index, 125

index.h, 273

indexFileName ibis::index, 127

indexSpec

ibis::bord, 56

ibis::mensa, 140

ibis::tabele, 224

ibis::table, 231

ibis::tabula, 245

init

ibis, 9

inRange

ibis::qContinuousRange, 178

ibis::qRange, 182

insert

array_t, 18

insertRaw

ibis::dictionary, 96

INT

ibis, 9

inUse

ibis::fileManager::storage, 116

irelic.h, 274

iroster.h, 274

isIndex

ibis::index, 127

is ValidToken

ibis::query, 191

keywords

ibis::keywords, 133 
limit

ibis::query, 191

loadIndex ibis::part, 169

locate ibis::roster, 211

logMessage ibis::query, 191

LONG

ibis, 9

lookforString ibis::part, 169

mapValues ibis::index, 128

markovSize ibis::bitvector, 40 ibis::bitvector64, 45

matchAny ibis::part, 169

mensa.h, 275

mergeBlock2

ibis::roster, 211

meshQuery.h, 275

metaTags

ibis::part, 169

nosharing

array_t, 18

OID

ibis, 9

operator $=$

ibis::bitvector, 40

operator[]

array_t, 18

ibis::resource, 206

ibis::tableList, 237

orderby

ibis::bord, 56

ibis::mensa, 140

ibis::query, 191

ibis::tabele, 224

ibis::table, 232

ibis::tabula, 245

orderPairs

ibis::query, 191

outerProduct

ibis, 9

parseNames

ibis::table, 232

parseNameValuePairs

ibis::resource, 206

parseQuery

ibis, 10

part

ibis::part, 165 part.h, 276

pastUse

ibis::fileManager::storage, 116

predicate.h, 277

PREFERRED_BLOCK_SIZE

const.h, 269

print

ibis::ambit, 21

ibis::bak, 24

ibis::bak2, 26

ibis::bin, 33

ibis::bylt, 66

ibis::direkte, 99

ibis::egale, 104

ibis::entre, 107

ibis::fade, 109

ibis::fuzz, 118

ibis::index, 128

ibis::keywords, 133

ibis::mesa, 143

ibis::moins, 148

ibis::pack, 151

ibis::pale, 154

ibis::range, 197

ibis::relic, 203

ibis::sapid, 212

ibis::sbiad, 214

ibis::slice, 217

ibis::zona, 255

ibis::zone, 258

printRange

ibis::qAnyAny, 176

ibis::qContinuousRange, 178

ibis::qExpr, 180

printSelected

ibis::query, 192

printSelectedWithRID

ibis::query, 192

processJoin

ibis::query, 192

purgeIndexFiles

ibis::part, 169

qExpr.h, 278

query

ibis::query, 189

query.h, 279

randomSize

ibis::bitvector, 40

ibis::bitvector64, 45

read

ibis::ambit, 21

ibis::bak, 24

ibis::bak2, 26

ibis::bin, 34

ibis::bitvector, 40 
ibis::bitvector64, 45

ibis::bylt, 66, 67

ibis::direkte, 99

ibis::egale, 104

ibis::fuzz, 118

ibis::index, 129

ibis::keywords, 134

ibis::pack, 152

ibis::pale, 154

ibis::part::barrel, 172

ibis::part::vault, 175

ibis::range, 198

ibis::relic, 203

ibis::resource, 206

ibis::ridHandler, 209

ibis::slice, 217, 218

ibis::zona, 256

ibis::zone, 258

readCSV

ibis::tablex, 239

ibis::tafel, 248

readKeyword

ibis::keywords, 134

readString

ibis::text, 251

readTDC

ibis::part, 169

readTermDocFile

ibis::keywords, 134

recordPages

ibis::fileManager, 112

recordVariable

ibis::compRange::barrel, 95

relic

ibis::relic, 202

remove

ibis::tableList, 237

removeComplexConditions

ibis::query, 192

reorder

ibis::bundles, 64

ibis::colDoubles, 70

ibis::colFloats, 72

ibis::colInts, 74

ibis::colLongs, 76

ibis::colUInts, 78

ibis::colULongs, 80

ibis::colValues, 92

ibis::part, 170

reserve

array_t, 18

reset

ibis::query::result, 195

resource.h, 280

resume

ibis::horometer, 119

rids.h, 280 rollback

ibis::part, 170

roster

ibis::roster, 210

rowCounts

ibis::bundle, 60

score

ibis::fileManager::roFile, 114

seek

ibis::part::barrel, 172

selectBytes

ibis::column, 87

selectDoubles

ibis::bord::column, 57

ibis::column, 87

selectLongs

ibis::bord::column, 57

ibis::column, 87

selectShorts

ibis::column, 87

selectUInts

ibis::text, 251

selectValues

ibis::column, 88

separateSimple

ibis::qExpr, 180

sequentialScan

ibis::query, 192

set

ibis::bitvector, 41

ibis::bitvector64, 46

setBases

ibis::index, 129

setBit

ibis::bitvector, 41

ibis::bitvector64, 46

setBoundaries

ibis::bin, 34

setMeshShape

ibis::part, 170

setMetaTags

ibis::part, 170

setSelectClause ibis::query, 192

setSize

ibis::bitvector, 41

setTable

ibis::query, 192

setWhereClause

ibis::query, 193

SHORT

ibis, 9

simplify

size ibis::qExpr, 180

array_t, 18 
sort

ibis::colDoubles, 70

ibis:: colFloats, 72

ibis::colInts, 74

ibis:: colLongs, 76

ibis::colUInts, 78

ibis::colULongs, 80

ibis::colValues, 92

sortEquiJoin

ibis::query, 193

sortRangeJoin

ibis::query, 193

stableSort

array_t, 18, 19

start

ibis::horometer, 119

startPositions

ibis::text, 251

std, 10

stop

ibis::horometer, 119

str

ibis::index, 130

sumBins

ibis::index, 129

sumBits

ibis::index, 129, 130

tab.h, 281

table.h, 281

tafel.h, 283

tellReal

ibis::part::vault, 175

TEXT

ibis, 9

THREAD_RWLOCK_INITIALIZER

const.h, 269

topk

array_t, 19

truncate

ibis::bundles, 64

truncateData

ibis::column, 88

tryGetFile

ibis::fileManager, 113

twister.h, 283

TYPE

ibis::qExpr, 180

TYPE_T

ibis, 9

UBYTE

ibis, 9

UINT

ibis, 9

ULONG

ibis, 9 undecidable

ibis::ambit, 21

ibis::bin, 34

ibis::direkte, 99

ibis::egale, 105

ibis::index, 130

ibis::mesa, 143

ibis::pack, 152

ibis::pale, 154

ibis::range, 198

ibis::zone, 258

UNKNOWN_TYPE

ibis, 9

unnamed

ibis::fileManager::storage, 116

USHORT

ibis, 9

util.h, 284

write

ibis::ambit, 22

ibis::bak, 24

ibis::bak2, 26

ibis::bin, 35

ibis::bitvector, 41

ibis::bylt, 67

ibis::egale, 105

ibis::entre, 107

ibis::fade, 109

ibis::fuzz, 118

ibis::index, 130

ibis::mesa, 144

ibis::moins, 148

ibis::pack, 152

ibis::pale, 155

ibis::range, 198

ibis::relic, 204

ibis::resource, 207

ibis::ridHandler, 209

ibis::sapid, 212

ibis::sbiad, 214

ibis::slice, 218

ibis::tablex, 239

ibis::tafel, 249

ibis::zona, 256

ibis::zone, 258

writeSorted

ibis::roster, 211

zona

ibis::zona, 255 